Computer Science \& Information Technology 

Natarajan Meghanathan

David C. Wyld (Eds)

\section{Computer Science \& Information Technology}

$5^{\text {th }}$ International Conference of Advanced Computer Science \& Information Technology (ACSIT 2017)

Sydney, Australia, June 24 25, 2017

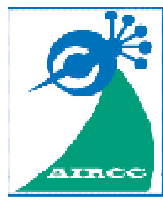




\section{Volume Editors}

Natarajan Meghanathan,

Jackson State University, USA

E-mail: nmeghanathan@jsums.edu

David C. Wyld,

Southeastern Louisiana University, USA

E-mail: David.Wyld@selu.edu

ISSN: $2231-5403$

ISBN: 978-1-921987-68-7

DOI : $10.5121 /$ csit.2017.70801 - 10.5121/csit.2017.70810

This work is subject to copyright. All rights are reserved, whether whole or part of the material is concerned, specifically the rights of translation, reprinting, re-use of illustrations, recitation, broadcasting, reproduction on microfilms or in any other way, and storage in data banks. Duplication of this publication or parts thereof is permitted only under the provisions of the International Copyright Law and permission for use must always be obtained from Academy \& Industry Research Collaboration Center. Violations are liable to prosecution under the International Copyright Law.

Typesetting: Camera-ready by author, data conversion by NnN Net Solutions Private Ltd., Chennai, India 


\section{Preface}

The $5^{\text {th }}$ International Conference of Advanced Computer Science \& Information Technology (ACSIT 2017) was held in Sydney, Australia, during June 24 25, 2017. The $5^{\text {th }}$ International Conference on Signal Image Processing and Multimedia (SIPM-2017), The $5^{\text {th }}$ International Conference on Foundations of Computer Science \& Technology (FCST-2017), The $9^{\text {th }}$ International Conference on Computer Networks \& Communications (CoNeCo-2017) and The $5^{\text {th }}$ International Conference of Managing Information Technology (CMIT-2017) was collocated with The $5^{\text {th }}$ International Conference of Advanced Computer Science \& Information Technology (ACSIT 2017). The conferences attracted many local and international delegates, presenting a balanced mixture of intellect from the East and from the West.

The goal of this conference series is to bring together researchers and practitioners from academia and industry to focus on understanding computer science and information technology and to establish new collaborations in these areas. Authors are invited to contribute to the conference by submitting articles that illustrate research results, projects, survey work and industrial experiences describing significant advances in all areas of computer science and information technology.

The ACSIT-2017, SIPM-2017, FCST-2017, CoNeCo-2017, CMIT-2017 Committees rigorously invited submissions for many months from researchers, scientists, engineers, students and practitioners related to the relevant themes and tracks of the workshop. This effort guaranteed submissions from an unparalleled number of internationally recognized top-level researchers. All the submissions underwent a strenuous peer review process which comprised expert reviewers. These reviewers were selected from a talented pool of Technical Committee members and external reviewers on the basis of their expertise. The papers were then reviewed based on their contributions, technical content, originality and clarity. The entire process, which includes the submission, review and acceptance processes, was done electronically. All these efforts undertaken by the Organizing and Technical Committees led to an exciting, rich and a high quality technical conference program, which featured high-impact presentations for all attendees to enjoy, appreciate and expand their expertise in the latest developments in computer network and communications research.

In closing, ACSIT-2017, SIPM-2017, FCST-2017, CoNeCo-2017, CMIT-2017 brought together researchers, scientists, engineers, students and practitioners to exchange and share their experiences, new ideas and research results in all aspects of the main workshop themes and tracks, and to discuss the practical challenges encountered and the solutions adopted. The book is organized as a collection of papers from the ACSIT-2017, SIPM-2017, FCST-2017, CoNeCo-2017, CMIT-2017.

We would like to thank the General and Program Chairs, organization staff, the members of the Technical Program Committees and external reviewers for their excellent and tireless work. We sincerely wish that all attendees benefited scientifically from the conference and wish them every success in their research. It is the humble wish of the conference organizers that the professional dialogue among the researchers, scientists, engineers, students and educators continues beyond the event and that the friendships and collaborations forged will linger and prosper for many years to come.

Natarajan Meghanathan

David C. Wyld 


\section{Organization}

\section{General Chair}

Natarajan Meghanathan, Jackson State University, USA Brajesh Kumar Kaushik, Indian Institute of Technology - Roorkee, India

\section{Program Committee Members}

\author{
Abdulhamit Subasi \\ Ahmad Rawashdeh \\ Ahmed Abdou \\ Ahmed Korichi \\ Akram Abdelqader \\ Alaa Hamami \\ Amine Laghrib \\ Amir Rajaei \\ Amir Rastegarnia \\ Amiya Kumar Tripathy \\ Ankit Chaudhary \\ Atallah Mahmoud AL-Shatnawi \\ Ayman El-Sayed \\ Azeddine Chikh \\ Carlo Sau \\ Chaker LARABI \\ Chin-chen Chang \\ Chuanzong Zhang \\ Dabin Ding \\ Elaheh Pourabbas \\ Emad Awada \\ Hacer Yalim Keles \\ Hamid Alasadi \\ Hassan Ugail \\ Hayet Mouss \\ Héctor Migallón \\ Hongzhi \\ Jamal El Abbadi \\ Jamel Hattay \\ Jun Zhang \\ Kemal Avci \\ Keneilwe Zuva \\ Keyvanpour M \\ Klimis Ntalianis \\ Mansour Y. Bader \\ Mario Henrique Souza Pardo
}

Effat University, Saudi Arabia

University of Central Missouri, United States

Al-Quds University, Palestine

University of Ouargla, Algeria

AL-Zaytoonah University of Jordan, Jordan

Princess Sumaya University for Technology, Jordan

Faculté des Sciences Beni-Mellal, Marocco

University of Velayat, Iran

University of Malayer, Iran

Edith Cowan University, Australia

Truman State University, USA

Al al-Byte University, Jordan

Menoufia University, Egypt

University of Tlemcen, Algeria

Università degli Studi di Cagliari, Italy

Université de Poitiers, France

Feng Chia University, Taiwan

Aalborg University, Denmark

University of Central Missouri, United States

National Research Council, Italy

Applied Science University, Jordan

Ankara University, Turkey

Basra University, Iraq

University of Bradford, UK

Batna Univeristy, Algeria

Miguel Hernández University, Spain

Harbin Institute of Technology, China

Mohammadia V University Rabat, Morocco

University of Tunis El Manar, Tunisia

South China University of Technology, China

Abant Izzet Baysal University, Turkey

University of Botswana, Botswana

Alzahra University, Iran

Athens University of Applied Sciences, Greece

Al-Balqa Applied University, Jordan

University of São Paulo, Brazil 
Mary Wilner

Maryam hajakbari

Masoud Fathi Kazerouni

Masoud Vejdannik

Matineh Shaker

Md Zakirul Alam Bhuiyan

Meng Ding

Miguel A. Rodriguez-Hernandez

Mimoun Hamdi

Mohamed Senouci

Mohamed-Khireddine Kholladi

Mohamedmaher Benismail

Mohammad Rawashdeh

Mohammadreza Balouchestani

Mohammed Al-Sarem R.

Mohammed Fatehy Soliman

Mokhtar Mohammadi

Mostafa Ashry

Mourchid mohammed Ibn

Naveed Ahmed

Noura Taleb

Ouafa Mah

Paulo Roberto Martins de Andrade

Petrellis N

Prakash Duraisamy

Raad Ahmed Hadi

Ramgopal Kashyap

Razieh malekhoseini

Saban Gulcu

Salem Nasri

Saltanat Meiramova

Samuel Baraldi Mafra

Shoeib Faraj

Supriya Karmakar

Taeghyun Kang

Touahni. Raja

Virginia Araujo

Wee kuok kwee

Wonjun Lee

Xiaofeng Wang

Xuechao Li

Yuanchang Sun

Yueying Kao

Yuying Shi

Zeba Khanam,

Zili Li
University of Southern California, USA

Islamic Azad University, Iran

University of Siegen, Germany

Iran University of Science \& Technology, Iran

Northeastern University, United States

Fordham University, USA

National Institutes of Health, USA

ITACA Universitat Politecnica de Valencia, Spain

Ecole Nationale d'Ingenieurs de Tunis, Tunisia

Université d'Oran 1 Ahmed Ben Bella, Algeria

Echahid Hamma Lakhdar University, Algeria

King saud University, Saudi Arabia

University of Central Missouri, United States

Indiana Purdue Fort Wayne University, USA

Taibah University, KSA

Suez Canal University, Egypt

Shahrood University of Technology, Iran

Alexandria University, Egypt

Tofail University Kenitra, Morocco

University of Sharjah, UAE

Badji Mokhtar University, Algeria

Ouargla University, Algeria

University of Regina, Canada

TEI of Thessaly, Greece

University of Central Missouri, United States

Iraqi University, Iraq

SISTec, India

Islamic Azad University, Iran

Necmettin Erbakan University, Turkey

Qassim University, Saudi Arabia

Seifullin Kazakh Agrotechnical University, Kazakhstan

Federal University of Parana (UFPR), Brazil

Institute of Higher Education of Miaad, Iran

Mellanox Technologies, USA

University of Central Missouri, United States

IBN Tofail University, Morocco

University of Vigo, Spain

Multimedia University, Malaysia

The University of Texas at San Antonio, USA

Bohai University, China

Auburn University, USA

Florida International University, USA

Chinese Academy of Sciences, China

North China Electric Power University, China

JSS Academy of Technical Education, India

Delft University of Technology, Netherland 


\section{Technically Sponsored by}

Computer Science \& Information Technology Community (CSITC)

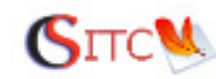

Networks \& Communications Community (NCC)

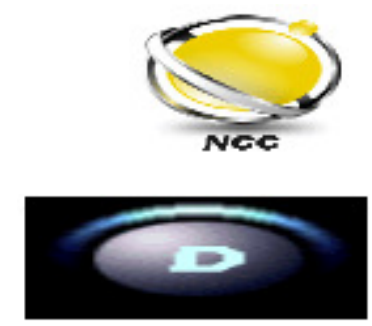

Digital Signal \& Image Processing Community (DSIPC)

\section{Organized By}

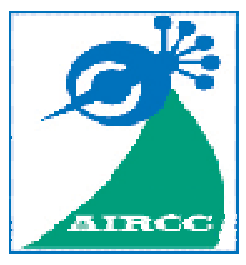

Academy \& Industry Research Collaboration Center (AIRCC) 


\section{TABLE OF CONTENTS}

\section{$5^{\text {th }}$ International Conference of Advanced Computer Science \& Information Technology (ACSIT 2017)}

Automated Usability Evaluation of E-Learning Websites in Saudi Arabia....... 01 - 10 Khalid Al-Omar

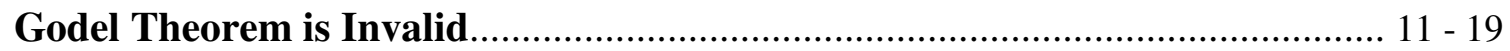

J. Ulisses Ferreira

Scalable and Efficient Pathsensitive Analysis Technique Scanning Many

Types of Vulnerability. $21-40$ Dongok Kang and Minsik Jin

Classification Algorithms for the Detection of the Primary Tumor Based on Microscopic Images of Bone Metastases. $41-56$ Slađan Kantar, Aleksandar Pluškoski, Igor Ciganovic and Jelena Vasiljević

Securing Information Systems A New Approach $115-128$

William R. Simpson

\section{$5^{\text {th }}$ International Conference on Signal Image Processing and Multimedia (SIPM-2017)}

A Collaborative Coding Framework of RS and H.264.

$57-70$

Jiejun $\mathrm{Hu}$ and Xilong Che

Building a Text-to-Speech System for Punjabi Language $71-87$

Rupinderdeep Kaur, R.K. Sharma and Parteek Kumar

\section{$5^{\text {th }}$ International Conference on Foundations of Computer Science \& Technology (FCST-2017)}

Kurtosis : Is it an Appropriate Measure to Compare the Extent of FatTailedness of the Degree Distribution for any two Realworld Networks?. $89-99$

Natarajan Meghanathan 


\section{$9^{\text {th }}$ International Conference on Computer Networks \& Communications (CoNeCo-2017)}

Dynamic Output Feedback Control for Sampled Data System with Actuator Saturation Using Quantized Measurements $101-114$

Liuwen Li, Wenlin Zou and Shumin Fei

\section{$5^{\text {th }}$ International Conference of Managing Information Technology (CMIT-2017)}

Investigating the Implication of Big Data Analytics on Consumer Finance in China. $129-135$

Jiwat Ram, Ding Ronggui and Hong Yang 


\title{
AUTOMATED USABILITY EVALUATION OF E-LEARNING WEBSITES IN SAUDI ARABIA
}

\author{
Khalid Al-Omar \\ Department of Information Systems, \\ King Abdulaziz University, Jeddah, Kingdom of Saudi Arabia
}

\begin{abstract}
Web usability is a significant factor in increasing user satisfaction, performance, trust, and loyalty. Web usability is particularly important for people who mostly depend on the website and for one reason or other cannot visit an institution, such as online distance education students. Accordingly, universities and educational websites need to determine the types of usability problems they have on their websites. However, far too little attention has been paid to providing detailed information regarding the types of specific usability problems that could be found on e-learning websites in general, and specifically, in the Kingdom of Saudi Arabia (KSA). The aim of this paper is to study and analyse the usability of university websites that offer distance education courses in the KSA. A total of 12 universities in Saudi Arabia were considered, which include 11 affiliated and one private university. The analysis of the data represents the level of usability of distance education websites. Results reveal that in Saudi Arabia, distance education websites are reliable, but violate basic usability guidelines.
\end{abstract}

\section{KEYWORDS}

University Websites, Credibility, Trustworthiness, Online Trust, Website Design, Saudi Arabia, Distance Education

Index Terms — Saudi Arabia, Distance Education, University Websites, Credibility, Trustworthiness, Online Trust, Website Design

\section{INTRODUCTION}

Today, the websites are crowded in both the Graphical User Interface and contents. This makes searching for information on universities web-based is complex. Therefore, sometimes university websites are become the obstacles to achieving that, once usability guidelines violated. Usability is an essential factor to measure the quality of the website. Usability becomes more vital once the system critical or for people who cannot visit an institution and depend mainly on the websitefor example, online distance education students. To ensure that students join their online distance education programs and not go elsewhere, universities should be certain that their websites are usable. Therefore, deanships of e-learning and distance education websites at universities require high usability.

Natarajan Meghanathan et al. (Eds) : ACSIT, SIPM, FCST, CoNeCo, CMIT - 2017

pp. 01- 10, 2017. (C) CS \& IT-CSCP 2017

DOI : $10.5121 /$ csit.2017.70801 
Computer Science \& Information Technology (CS \& IT)

Usability is one of the most important characteristics of any website. If the website is not usable, no one going to use it. Usability can make users comfortable interacting with the website, registering their personal information, and then become loyal users [1]. Usability is defined by ISO 9241-11, as "the extent to which a product can be used by specified users to achieve specified goals with effectiveness, efficiency, and satisfaction in a specified context of use" [2].

The remainder of the paper is organized as follows: First, there is an overview of usability evaluation, presented in Section 2; then, a brief description of distance education in the KSA is given in Section 3. In Section 4, there is a brief description of relevant previous studies and a literature review. In Section 5, objectives of this work are presented. Section 6 presents the methodology used. Websites evaluation automated tools is presented in Section 7. In Section 8, tool-based results are provided; in Section 9, there is a discussion of these results. Finally, conclusions and future work are discussed in Section 10.

\section{USABILITY EVALUATION}

There are different types of evaluation methods exists to examine usability. These methods categorized into three categories based on how the usability problems are identified:

- Expert evaluation methods: include methods that involves one or a group of experts in the process of identifying usability problems. Common usability method related to this category is a Heuristic evaluation[1, 3, 4] and Cognitive walkthroughs[5, 6] method.

- User evaluation methods: include methods that involve users in the process of identifying usability problems. Common examples for this category is a low fidelity prototype and involving users in focus user groups or controlled laboratory sessions to provide usability feedback[7-9].

- Tool-based evaluation methods: include methods that involve software in the process of identifying usability problems. Common usability tools related to this category are Qualidator, Website Grader, Search Engine Optimization (SEO), and Web page Analyser.

\section{A BRIEF Note AbOUT Distance EDUCATION IN THE KSA}

In the present day, the Kingdom of Saudi Arabia (KSA) has witnessed growth in the number of universities in the country. According to the Ministry of Higher Education in the KSA, there are 35universities in Saudi Arabia. There are 25 government universities,10private universities, and one university focusing exclusively on graduate education and research-King Abdullah University of Science and Technology (KAUST). Among the 36 universities, only 11 government universities have been authorized by the Ministry of Higher Education to offer distance education courses ranging from bachelors' to masters' degrees. On the other hand, only one private university (the Arab Open University) has been accredited by the Ministry of Higher Education. In this study, all 12 universities were considered, evaluated, and analysed (Table 1). 
TABLE 1: YEAR OF ESTABLISHMENT OF E-LEARNING AND DISTANCE EDUCATION DEANSHIPS AT UNIVERSITIES IN THE KSA

\begin{tabular}{|l|l|l|l|}
\hline \multirow{2}{*}{ No } & \multirow{2}{*}{ University } & \multicolumn{2}{|c|}{ Year of Establishment } \\
\cline { 3 - 4 } & & Gregorian & Hijri \\
\hline 1 & King Abdulaziz University (KAU) & 2002 & 1423 \\
\hline 2 & Islamic University in Madinah & 1961 & 1381 \\
\hline 3 & IMAUM & 2007 & 1428 \\
\hline 4 & King Faisal University (KFU) & 2009 & 1430 \\
\hline 5 & TAIBUAHU & 2005 & 1426 \\
\hline 6 & Taif University (TU) & 2011 & 1432 \\
\hline 7 & Jazan University (JAZANU) & 2009 & 1430 \\
\hline 8 & Aljouf University (JU) & 2007 & 1428 \\
\hline 9 & Najran University (NU) & 2011 & 1432 \\
\hline 10 & University of Dammam (UD) & 2010 & 1430 \\
\hline 11 & Saudi Electronic University (SEU) & 2011 & 1432 \\
\hline 12 & Arab Open University & 2002 & 1423 \\
\hline
\end{tabular}

Table 2 shows the number of e-learning students enrolled in each university for male and female.

TABLE 2: NUMBER OF ONLINE DISTANCE EDUCATION STUDENTS IN THE KSA 2014

\begin{tabular}{|l|l|l|l|l|}
\hline No & University & Male & Female & Total \\
\hline 1 & King Abdulaziz University (KAU) & 1,959 & 1,909 & 3,868 \\
\hline 2 & Islamic University in Madinah & 700 & 0 & 700 \\
\hline 3 & IMAUM & 5,156 & 3,733 & 8,889 \\
\hline 4 & King Faisal University (KFU) & 5,911 & 6,901 & 1,2812 \\
\hline 5 & TAIBUAHU & 1,530 & 1,713 & 3,243 \\
\hline 6 & Taif University (TU) & 2,014 & 1,041 & 3,055 \\
\hline 7 & Jazan University (JAZANU) & 876 & 810 & 1,686 \\
\hline 8 & Aljouf University (JU) & 199 & 66 & 265 \\
\hline 9 & Najran University (NU) & 1,005 & 977 & 1,982 \\
\hline 10 & University of Dammam (UD) & 957 & 1,241 & 2,198 \\
\hline 11 & Saudi Electronic University (SEU) & 4,490 & 2,771 & 7,261 \\
\hline 12 & Arab Open University & 1,914 & 2,340 & 4,254 \\
\hline
\end{tabular}

\section{LITERATURE REVIEW}

Evaluations of the usability of websites have been the subject of many research projects, such as e-learning (e.g. [10, 11]), e-government (e.g. [12, 13]), e-commerce (e.g. [14-18]), mobile website interfaces (e.g. [19, 20]), m-commerce (e.g. [21, 22]), and virtual reality and augmented reality (e.g. [23, 24]). In addition, there are some automated usability evaluation websites conducted.

Alexander et al. [25] evaluated the usability and accessibility of three UK e-government websites and investigated if these two related. Two automated usability evaluation tools used namely: Bobby and Lift. Based on the automated evaluation selected websites evaluated by heuristics and walkthrough evaluation. Their study showed that UK e-government is high compliance with WCAG and a relatively low usability rating. 
Junaini et al. [26] evaluate three African countries websites by using WebQual tool. The tool found that the HTML elements in handcoded pages and presented the highest number of accessibility problems.

Mustafa et al. [27] utilized HTML Toolbox and Webpage Analyser to evaluate nine university Jordanian websites. Their results showed that the overall usability level of the studied Websites is acceptable.

Sukhpuneet et al. [28] make use of two automated tools namely: Site Analyser and Qualidator tool to evaluate educational universities of Punjab and provides their ranks according to evaluation criteria. Their results showed that websites designers could focus on specific features where are they lacking.

Ivory study et al. [29] used three usability tools namely: W3C HTML, UsableNet life, and WatchFire Boby. Their results showed that the tools helped designers to identify a larger number of usability problems.

Oliha et al. [30] evaluated the usability of two Polytechnics websites in Nigeria. They used HTML toolbox and webpage analyzer. Their study revealed that the overall usability level is acceptable, but there are some weaknesses in the phase of design and interfaces.

\section{OBJECTIVES}

The main objective of this study is to examine the web usability of e-learning and distance education deanships websites at universities in the KSA, compare the online distance education websites of universities in the KSA, and then offer suggestions for the design of an ideal online distance education website for a university to increase the site'susability.

\section{METHODOLOGY}

The usability evaluation conducted by a human (users and experts) evaluate the external attributes of the website, rather than its internal attributes (such as webpage download time). External attributes depend on the website and its usage, while the internal attributes of the website depend on how the website has been designed and developed [31]. In this study, automated tools used to evaluate the internal attributes.

\section{WebSite EVAluATiOn USING AUTOMATED TOOLS}

The evaluation method utilized to evaluate the usability of the e-learning websites of Saudi universities by using automated tools. There are many automated evaluation tools available to assess different usability attributes. In this study, three different evaluation automated tools were chosen to analyse different usability factors such as performance, load time, navigation, mobile friendly, and user satisfaction. SEO, accessibility, and security will help to increase user satisfaction. The selected website evaluation tools are: 
- The webpage analyser 0.98 is a free tool for Website Optimization utilized to measure the website performance tool and webpage speed analysis to improve a website's performance.

- The Qualidator Tool is a free online tool that measures website against website performance, accessibility, SEO, and usability.

- The website Grader is a free online tool that grade website against website performance, Mobile, SEO, and Security.

Table 3 shows the Performance, Accessibility, Mobile, Search Engine Optimization (SEO), Usability, and Page Analysis.

TABLE 3: WEBSITE EVALUATION AUTOMATED TOOLS

\begin{tabular}{|l|c|c|c|}
\hline Criteria & $\begin{array}{c}\text { WebPage } \\
\text { Analyser }\end{array}$ & Qualidator & $\begin{array}{l}\text { Website } \\
\text { Grader }\end{array}$ \\
\hline Performance & $\sqrt{ }$ & $\sqrt{ }$ & $\sqrt{ }$ \\
\hline Accessibility & & $\sqrt{ }$ & \\
\hline Mobile & & $\sqrt{ }$ & $\sqrt{ }$ \\
\hline SEO & & $\sqrt{ }$ & \\
\hline Security & & $\sqrt{ }$ \\
\hline Usability & & & \\
\hline $\begin{array}{l}\text { Page } \\
\text { analysis }\end{array}$ & $\sqrt{ }$ & & \\
\hline
\end{tabular}

\section{RESUlTS OF AUTOMATED TOOLS}

- Results of WebPage Analyser Tool

Testing website download speed and the size of the webpage influences the usability of any website. The data obtained used only to represent the extent and the level of website download speed and size of the webpage possessed by the university websites in Saudi Arabia. In this study, the total size of the website, the total size of images, the percentage of images in the total size, and the download times have been collected. The results obtained from the WebPage Analyser are presented in Table 4. 
TABLE 4: CLASSIFICATION OF WEBSITES BY WEBPAGE SIZE AND DOWNLOAD SPEED

\begin{tabular}{|l|l|l|l|l|}
\hline $\begin{array}{l}\text { Name of the } \\
\text { university }\end{array}$ & $\begin{array}{l}\text { Total size } \\
\text { of the } \\
\text { website }\end{array}$ & $\begin{array}{l}\text { Total size } \\
\text { of the } \\
\text { images }\end{array}$ & $\begin{array}{l}\text { Percentage } \\
\text { of images in } \\
\text { total size }\end{array}$ & $\begin{array}{l}\text { Download } \\
\text { time at 56K } \\
\text { connections }\end{array}$ \\
\hline KAU & 1885781 & 930851 & 55 & 393.63 \\
\hline IU & 3013113 & 451273 & 98 & 949.76 \\
\hline IMAUM & 284501 & 61956 & 6 & 61.10 \\
\hline KFU & 1017485 & 734151 & 19 & 212.18 \\
\hline TAIBUAHU & 974124 & 789597 & 74 & 212.74 \\
\hline TU & 2155286 & 1250190 & 44 & 441.35 \\
\hline JAZANU & 3126833 & 458363 & 99 & 969.56 \\
\hline JU & 2461388 & 2273857 & 72 & 514.35 \\
\hline NU & 457998 & 448225 & 11 & 93.88 \\
\hline UD & 2528954 & 2039003 & 84 & 525.42 \\
\hline SEU & 629 & 0 & 0 & 0.53 \\
\hline ARABOU & 633 & 0 & 0 & 0.53 \\
\hline
\end{tabular}

The web optimization's WebPage Speed Report has the connection rate starting from 1.44 Mbps to $14.4 \mathrm{~K}$. According to the usability guidelines [32], the optimal download time for a homepage is 10 seconds. So, for better download speed, it is suggested to design $45 \mathrm{~kb}$ to $55 \mathrm{~kb}$-sized homepages. Table 5 shows that only two universities fall in the $<10$ seconds category, and only one university fall under the $>100$ seconds category. Other homepages of universities in Saudi Arabia fall under the $>200$ seconds category. Saudi Arabia has pictures, which occupies nearly 70 to 98 percent of the overall website size.

\section{- $\quad$ Results of Qualidator Tool}

The result of Qualidator Tool is shown in Table 5. This tool measured against the key usability, accessibility, SEO, and Overall. Unfortunately, TAIBUAHU university website could not be tested. Since, the error message returned by the server indicated that the operation has timed out. From the result of the Qualidator Websites analyser tool, IMAUM University score (82.5\%) more points than other university websites in terms usability. Whereas UD, JU, and NU universities websites score respectively $(81.7 \%, 80.8 \%, 80.6 \%)$ more points in terms accessibility. Both JU and IU universities websites score (79.6\%) more in SEO. Overall JU university websites score $(78.5 \%)$ more points than other universities. Followed by NU university websites score $(77.3 \%)$ more points than other universities. 
TABLE 5: RESULTS OF WEBSITE QUALIDATOR TOOL

\begin{tabular}{|l|l|l|l|l|l|}
\hline No & $\begin{array}{l}\text { University } \\
\text { Website }\end{array}$ & Usability & Accessibility & $\begin{array}{l}\text { SEO Search Engine } \\
\text { Optimization) }\end{array}$ & Overall \\
\hline 1 & KAU & $68.6 \%$ & $67.2 \%$ & $68.1 \%$ & $72.8 \%$ \\
\hline 2 & IU & $76.5 \%$ & $76.3 \%$ & $79.9 \%$ & $75.7 \%$ \\
\hline 3 & IMAUM & $82.5 \%$ & $74.4 \%$ & $76.6 \%$ & 75.3 \\
\hline 4 & KFU & $72.8 \%$ & $69.0 \%$ & $61.4 \%$ & $70 \%$ \\
\hline 5 & TU & $66.5 \%$ & $65.6 \%$ & $67.3 \%$ & $66.2 \%$ \\
\hline 6 & JAZANU & $68.9 \%$ & $65.3 \%$ & $65.3 \%$ & $67.7 \%$ \\
\hline 7 & JU & $77.9 \%$ & $80.8 \%$ & $79.6 \%$ & $78.5 \%$ \\
\hline 8 & NU & $75.9 \%$ & $80.6 \%$ & $75.2 \%$ & $77.3 \%$ \\
\hline 9 & UD & $78.3 \%$ & $81.7 \%$ & $70.4 \%$ & $76.4 \%$ \\
\hline 10 & SEU & $77.7 \%$ & $78.5 \%$ & $68.3 \%$ & $75.3 \%$ \\
\hline 11 & ARABOU & $66.7 \%$ & $65.8 \%$ & $71.9 \%$ & $66.3 \%$ \\
\hline
\end{tabular}

- $\quad$ Results of Website Grader Tool

The result of Website Grader Tool is shown in Table 6. This tool measured against the key performance, Mobile, SEO, Security, and Overall. Unfortunately, TAIBUAHU university website could not be tested. Since, the error message returned by the server indicated that the operation has timed out. From the result of the Website Grader tool, UD and KFU University score (80) more points than other university websites in Overall. In addition, four universities (TU, JAZANU, SEU, and ARABOU) score less than (50). For Mobile design, four universities score zero, whereas, the other universities score full mark. Seven universities score less than half regarding the performance term. UD and SE Universities score high performance (30, 26 respectively) comparing with the other universities.

TABLE 6: RESULTS OF WEBSITE GRADER TOOL

\begin{tabular}{|l|l|l|l|l|l|}
\hline University & Performance & Mobile & SEO & Security & Overall \\
\hline KAU & $14 / 30$ & $30 / 30$ & $15 / 30$ & $10 / 10$ & 69 \\
\hline IU & $14 / 30$ & $30 / 30$ & $15 / 30$ & $10 / 10$ & 69 \\
\hline IMAUM & $14 / 30$ & $30 / 30$ & $20 / 30$ & $10 / 10$ & 74 \\
\hline KFU & $14 / 30$ & $30 / 30$ & $25 / 30$ & $10 / 10$ & 79 \\
\hline TU & $14 / 30$ & $0 / 30$ & $10 / 30$ & $10 / 10$ & 34 \\
\hline JAZANU & $19 / 30$ & $0 / 30$ & $10 / 30$ & $0 / 10$ & 29 \\
\hline JU & $20 / 30$ & $30 / 30$ & $15 / 30$ & $0 / 10$ & 65 \\
\hline NU & $12 / 30$ & $30 / 30$ & $30 / 30$ & $0 / 10$ & 72 \\
\hline UD & $30 / 30$ & $30 / 30$ & $10 / 30$ & $10 / 10$ & 80 \\
\hline SEU & $26 / 30$ & $0 / 30$ & $10 / 30$ & $10 / 10$ & 46 \\
\hline ARABOU & $13 / 30$ & $0 / 30$ & $10 / 30$ & $0 / 10$ & 23 \\
\hline
\end{tabular}

\section{RESULTS AND DISCUSSION}

Table 12 presents a summary of the score for each of the 12 university websites. For the "design" factor, only the SU and KAU website scored more than half of the total, where the rest scored half or less. The SEU, NU, KAU, and IU university websites achieved the highest score (61 percent) for the "easy to use" factor, where the rest of the websites scored less than half. This indicates that half of the university websites have usability problems on their websites. On the 
other hand, most university websites achieved a high score on "reliability factors." The JU university website scored the lowest for both the "validity" and "expertise" factors, followed by the NU university website. Three universities (KAU, KFU, and SEU) websites achieved full marks in the expertise area.

\section{CONCLUSION AND FUTURE WORK}

The purpose of the current study was to examine the web usability of distance education websites in Saudi Arabia universities. The results of this investigation show that university websites are reliable and are designed well, but violate basic accessibility, and usability guidelines. Therefore, university websites in Saudi Arabia should be required to be evaluated periodically using established criteria such as usability, accessibility, and credibility. Consequently, this will help the universities improve their websites to meet users' needs. The current study should be repeated using the user evaluation method.

\section{REFERENCES}

[1] J. Nielsen, "How to conduct a heuristic evaluation," retrieved November, vol. 10, 2001.

[2] T. Jokela, N. Iivari, J. Matero, and M. Karukka, "The standard of user-centered design and the standard definition of usability: analyzing ISO 13407 against ISO 9241-11," in Proceedings of the Latin American conference on Human-computer interaction, 2003, pp. 53-60.

[3] J. Nielsen and R. Molich, "Heuristic evaluation of user interfaces," in Proceedings of the SIGCHI conference on Human factors in computing systems, 1990, pp. 249-256.

[4] E. T. Hvannberg, E. L.-C. Law, and M. K. Lérusdóttir, "Heuristic evaluation: Comparing ways of finding and reporting usability problems," Interacting with computers, vol. 19, pp. 225-240, 2007.

[5] P. G. Polson, C. Lewis, J. Rieman, and C. Wharton, "Cognitive walkthroughs: a method for theorybased evaluation of user interfaces," International Journal of man-machine studies, vol. 36, pp. 741$773,1992$.

[6] M. H. Blackmon, P. G. Polson, M. Kitajima, and C. Lewis, "Cognitive walkthrough for the web," in Proceedings of the SIGCHI conference on human factors in computing systems, 2002, pp. 463-470.

[7] J. Brooke, "SUS-A quick and dirty usability scale," Usability evaluation in industry, vol. 189, pp. 4-7, 1996.

[8] G. Perlman, "Web-based user interface evaluation with questionnaires," Retrieved March, vol. 1, p. 2003, 2001.

[9] T. S. Tullis and J. N. Stetson, "A comparison of questionnaires for assessing website usability," in Usability Professional Association Conference, 2004, pp. 1-12.

[10] K. Orfanou, N. Tselios, and C. Katsanos, "Perceived usability evaluation of learning management systems: Empirical evaluation of the System Usability Scale," The International Review of Research in Open and Distributed Learning, vol. 16, 2015. 
[11] M. Alshammari, R. Anane, and R. J. Hendley, "Design and Usability Evaluation of Adaptive elearning Systems Based on Learner Knowledge and Learning Style," in Human-Computer Interaction, 2015, pp. 584-591.

[12] Z. Huang and M. Benyoucef, "Usability and credibility of e-government websites," Government Information Quarterly, vol. 31, pp. 584-595, 2014.

[13] H. Gull and S. Z. Iqbal, "Usability Evaluation of E-Government Websites in Saudi Arabia by Cognitive Walkthrough," Design Solutions for User-Centric Information Systems, p. 297, 2016.

[14] B. Fogg, J. Marshall, O. Laraki, A. Osipovich, C. Varma, N. Fang, et al., "What makes Web sites credible?: a report on a large quantitative study," in Proceedings of the SIGCHI conference on Human factors in computing systems, 2001, pp. 61-68.

[15] C. N. Wathen and J. Burkell, "Believe It or Not: Factors Influencing Credibility on the Web," JOURNAL OF THE AMERICAN SOCIETY FOR INFORMATION SCIENCE AND TECHNOLOGY, vol. 53, pp. 134-144, 2002.

[16] F. Alsudani and M. Casey, "The effect of aesthetics on web credibility," in Proceedings of the 23rd British HCI Group Annual Conference on People and Computers: Celebrating People and Technology, 2009, pp. 512-519.

[17] L. Song, J. Lai, and J. Li, "Identifying Factors Affecting Individual Perceived Credibility on SNS," in Proceedings of the The 3rd Multidisciplinary International Social Networks Conference on SocialInformatics 2016, Data Science 2016, 2016, p. 2.

[18] J. F. George, G. Giordano, and P. A. Tilley, "Website credibility and deceiver credibility: Expanding Prominence-Interpretation Theory," Computers in Human Behavior, vol. 54, pp. 83-93, 2016.

[19] A. S. Tsiaousis and G. M. Giaglis, "Mobile websites: usability evaluation and design," International Journal of Mobile Communications, vol. 12, pp. 29-55, 2014.

[20] B. C. Zapata, J. L. Fernández-Alemán, A. Idri, and A. Toval, "Empirical studies on usability of mHealth apps: A systematic literature review," Journal of medical systems, vol. 39, pp. 1-19, 2015.

[21] A. Hussain, E. O. Mkpojiogu, F. A. A. Nifa, M. N. M. Nawi, and A. Hussain, "Usability evaluation techniques in mobile commerce applications: a systematic review," in AIP Conference Proceedings, 2016, p. 020049.

[22] A. Hussain, E. O. Mkpojiogu, and F. M. Kamal, "A Systematic Review on Usability Evaluation Methods for M-Commerce Apps," Journal of Telecommunication, Electronic and Computer Engineering (JTEC), vol. 8, pp. 29-34, 2016.

[23] P. Rane, H. Kim, J. L. Marcano, and J. L. Gabbard, "Virtual Road Signs: Augmented Reality Driving Aid for Novice Drivers," in Proceedings of the Human Factors and Ergonomics Society Annual Meeting, 2016, pp. 1750-1754.

[24] A. S. Merians, D. Jack, R. Boian, M. Tremaine, G. C. Burdea, S. V. Adamovich, et al., "Virtual reality-augmented rehabilitation for patients following stroke," Physical therapy, vol. 82, pp. 898$915,2002$.

[25] A. Rukshan and A. Baravalle, "Automated Usability Testing: Analysing Asia Web Sites," arXiv preprint arXiv:1212.1849, 2012. 
[26] S. Junaini, "Navigation design and usability evaluation of the Malaysian public university websites," in Proceedings of the Second National Conference on Cognitive Science CSC, 2002, pp. 181-189.

[27] S. H. Mustafa and L. F. Al-Zoua'bi, "Usability of the academic websites of Jordan's universities an evaluation study," in Proceedings of the 9th International Arab Conference for Information Technology, 2008, pp. 31-40.

[28] S. Kaur, K. Kaur, and P. Kaur, "Analysis of website usability evaluation methods," in Computing for Sustainable Global Development (INDIACom), 2016 3rd International Conference on, 2016, pp. 1043-1046.

[29] M. Y. Ivory and A. Chevalier, "A study of automated web site evaluation tools," University of Washington, Department of Computer Science2002, 2002.

[30] F. Oliha, "Web portal usability among Nigerian university students: A case study of University of Benin, Nigeria," Nigerian Journal of Technology, vol. 33, pp. 199-206, 2014.

[31] G. Brajnik, "Automatic web usability evaluation: what needs to be done," in Proc. Human Factors and the Web, 6th Conference, 2000.

[32] J. Nielsen, "Designing web usability: the practice of simplicity New Riders Publishing," Indianapolis, Indiana, 2000. 


\title{
GÖDEL THEOREM IS INVALID
}

\author{
J. Ulisses Ferreira
}

Trv Pirapora 36 Costa Azul, 41770-220, Salvador, Brazil

\begin{abstract}
This short and informal article shows that there exists some deductive system that proves that arithmetic is both sound and complete. First, it shows that there exists some four-valued logical system that plays the same role, by presenting a four-valued logic and informally introducing a four-valued Prolog programming language. Finally, it observes that some Boolean formal system can also prove that arithmetic is both sound and complete.
\end{abstract}

\section{KEYWORDS}

Gödel, incompleteness theorem, four-valued logic, logic, computability theory, philosophy of computer science

\section{INTRODUCTION}

In 1931, Kurt Gödel demonstrated that Hilbert program[1] would not work for mathematics[2].

On the other hand, in the nineties, the present author started inserting a third value called "unknown" in Plain[3], i.e. a programming language that he was designing at that time. The unknown constant was theoretically referred to as $u u$ since the end of nineties. Later, a fivevalued logic was introduced containing the values in $\{t t, f f, u u, i i, k k\}$. In 2004, the same logic was published as a journal article[4] and a seven-valued logic was also published in a Conference in San Diego[5], adding the values $\{f i$, it\} ("false or inconsistent", "inconsistent or true", respectively) for being able to be used together with the same uncertainty model that had been proposed during the present author's Master course in 1990. The seven-valued logic that makes use of that uncertainty model permits that, during the computation, as the system obtains novel pieces of information, variables change their values. An example of this is the paternity test: before the discovery of the DNA test, it was possible to conclude whether a child was a daughter or son of a particular man by hereditary physical characteristics. However, there was always uncertainty up to some extent. The uncertainty factor could be represented by $u u$ (unknown), at least as an initial state of some variable. Since the DNA test was discovered, all variables which represent the hypothesis of being the child's father should change their states from $u u$ to either $k k$ or $t t$ or ff.

As part of the present author's previous contribution, the $k k$ value means "knowable", and it is usable when something is not already known, but it is already known that it is consistent. It can either be true or false but not both. It can be known by God or someone else or some machine, for instance, but it is not already known by the machine which is deductively reasoning, or by the Natarajan Meghanathan et al. (Eds) : ACSIT, SIPM, FCST, CoNeCo, CMIT - 2017 
person who is deductively doing, and it may be unknown forever, but at least its consistency is guaranteed. This is the meaning of the $k k$ value, which fits in the referred uncertainty model when a variable thresholds collapse: False $=$ True, which means that there is nothing strictly between the False and the True thresholds. In the above example of the paternity test, uu used to represent the initial state before the discovery of the DNA test, whereas $k k$ represents the initial state given the existence of the DNA test, but before knowing the result of a particular DNA test, either $f f$ or $t t$.

Individually and previously, Kleene, Łukasiewicz and Priest proposed their three-valued logics. In 1977, Nuel Belnap (1930-) had proposed his logic on four values[6]. What was observed several years ago is that Gödel's proof may not work together with some logics that have more than three values. The four necessary values mean "true", "false", "unknown" and "inconsistent", or similar meanings. That is, at least these four values and meanings. The latter two values correspond to $N$ (none) and $B$ (both) in the four-valued Belnap's logic, respectively, and correspond to $u u$ and $i i$, respectively, in both referred logics of the present author, as well as in his four-valued logic presented in this article, and the four-valued Prolog[7] also described here.

The problem Gödel introduced was due to existing self-references and paradoxes, which made propositions of arithmetic result in both true and false, together with the observation that any proof over mathematics was also a mathematical object itself. However, Boolean logics are clearly unable to permit that formal systems capture the problem pointed out by Gödel in his theorem. That is, no binary formal system can capture it.

One condition for a four-valued formal system being able to prove all true propositions, and only the true propositions, is certainly that it has the same results of the classical logic, except where one or two operands have values other than true and false. In any proof, a result here is the true value only, and do not include values such as "unknown". Belnap's four-valued logic does not fail under this condition, for, although $(B \vee N=T)$, where $T$ represents the true value, the values of the operands are " $B$ " and " $N$ ", which are not Boolean.

In the present author's $\mathrm{PhD}$ thesis, there was a kind of typo in the truth-table for the specific case $f f \leftrightarrow f f$, which results in $t t$ in the five-valued logic but $f f$ was written instead: a kind of mistake in only one of the two truth-tables for the equivalence operation. However, taking this into account, and by using only one of those equivalence operations, that five-valued logic suffices regarding that condition. Moreover, in 2007, having written a program which seems to be correct, it was checked whether such a four-valued logic exists, and its computation resulted in several logics, where one of them was Belnap's logic. The 12th solution written by the program computation was the logic which was the most interesting. In 2011, one could not claim the authorship of that fourvalued logic, but the referred truth-tables are the following:

Table 1. The present author's four-valued logic true table

\begin{tabular}{c|c}
$A$ & $\neg A$ \\
\hline$U$ & $I$ \\
$F$ & $T$ \\
$T$ & $F$ \\
$I$ & $U$
\end{tabular}

\begin{tabular}{c|cccc}
$A$ & $U$ & $F$ & $T$ & $I$ \\
\hline$U$ & $U$ & $F$ & $U$ & $U$ \\
$F$ & $F$ & $F$ & $F$ & $F$ \\
$T$ & $U$ & $F$ & $T$ & $I$ \\
$I$ & $U$ & $F$ & $I$ & $I$
\end{tabular}

\begin{tabular}{c|cccc}
$V$ & $U$ & $F$ & $T$ & I \\
\hline$U$ & $U$ & $U$ & $T$ & $I$ \\
$F$ & $U$ & $F$ & $T$ & $I$ \\
$T$ & $T$ & $T$ & $T$ & $T$ \\
I & I & I & $T$ & I
\end{tabular}

\begin{tabular}{c|cccc}
$\rightarrow$ & $\mathrm{U}$ & $\mathrm{F}$ & $\mathrm{T}$ & $\mathrm{I}$ \\
\hline $\mathrm{U}$ & $\mathrm{T}$ & $\mathrm{T}$ & $\mathrm{T}$ & $\mathrm{T}$ \\
$\mathrm{F}$ & $\mathrm{T}$ & $\mathrm{T}$ & $\mathrm{T}$ & $\mathrm{T}$ \\
$\mathrm{T}$ & $\mathrm{U}$ & $\mathrm{F}$ & $\mathrm{T}$ & $\mathrm{T}$ \\
$\mathrm{I}$ & $\mathrm{F}$ & $\mathrm{U}$ & $\mathrm{T}$ & $\mathrm{T}$
\end{tabular}

\begin{tabular}{l|llll}
$\leftrightarrow$ & $\mathrm{U}$ & $\mathrm{F}$ & $\mathrm{T}$ & $\mathrm{I}$ \\
\hline $\mathrm{U}$ & $\mathrm{T}$ & $\mathrm{F}$ & $\mathrm{F}$ & $\mathrm{F}$ \\
$\mathrm{F}$ & $\mathrm{F}$ & $\mathrm{T}$ & $\mathrm{F}$ & $\mathrm{F}$ \\
$\mathrm{T}$ & $\mathrm{F}$ & $\mathrm{F}$ & $\mathrm{T}$ & $\mathrm{F}$ \\
$\mathrm{I}$ & $\mathrm{F}$ & $\mathrm{F}$ & $\mathrm{F}$ & $\mathrm{T}$
\end{tabular}


Section 2 dedicates to Gödel theorem and his proof, and a system for capturing all possible results is informally described. In section 3, a four-valued Prolog programming language is briefly described, whereas section 4 contais the conclusions.

\section{ON GÖDEL THEOREM}

The set of all propositions on arithmetical true is written for the two Boolean values, but that set could be complete but cannot be sound, i.e. it is clearly inconsistent. However, the present author writes an external layer with an external view of that set, as well as an internal layer. The former layer is written with four or more values. It interprets that set and, thus, both layers together form a formal system where the two-valued system is the server while the four-valued system is the client. The external layer makes use of the internal one. The system with at least four values is pretty simple and works in the following manner:

Whenever one attempts to prove that a proposition is true and the two-valued system results in true, the external layer still tries to prove that the proposition is false: If the two-valued system results in true, the external layer results in $i i$, the inconsistent value. However, on the other hand, if the two-valued system results in false instead, the external layer results in true.

Whenever one attempts to prove that a proposition is true and the two-valued system results in false, the external layer still tries to prove that the proposition is false: If the two-valued system results in false, the external layer results in $u u$, the unknown value. However, on the other hand, if the two-valued system results in true instead, the external layer results in false.

In other words, the meaning of a two-valued internal query is only the attempt to prove, which either succeeds or not. In this way, the whole formal system is clearly sound and complete. In 1997, the present author wrote a three-valued Prolog which he called Kleene at that time and Globallog in the following year[8] for becoming more modest, and the same language is the subject of one of the chapters of his PhD thesis. The system described above in this section can be more clearly written in a Pascal-like language style as follows:

1. An algorithm in Pascal-like language

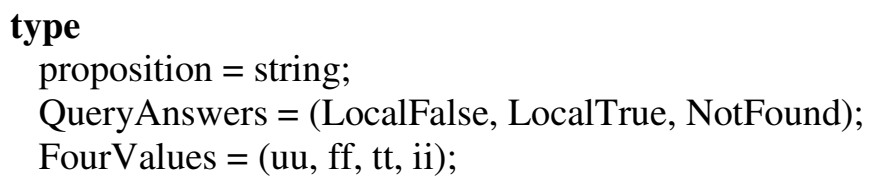

function TryProposition2v(p: proposition, q: QueryAnswers): boolean; begin

(*any polynomial search algorithm with unification for checking whether the proposition $\mathrm{p}$ is true. Alternatively, this function also returns the information that the search algorithm has been unable to answer whether the proposition $p$ is true or false with respect to the current state of the knowledge base. In this case, where no unification has been found, the result is false.

*) 


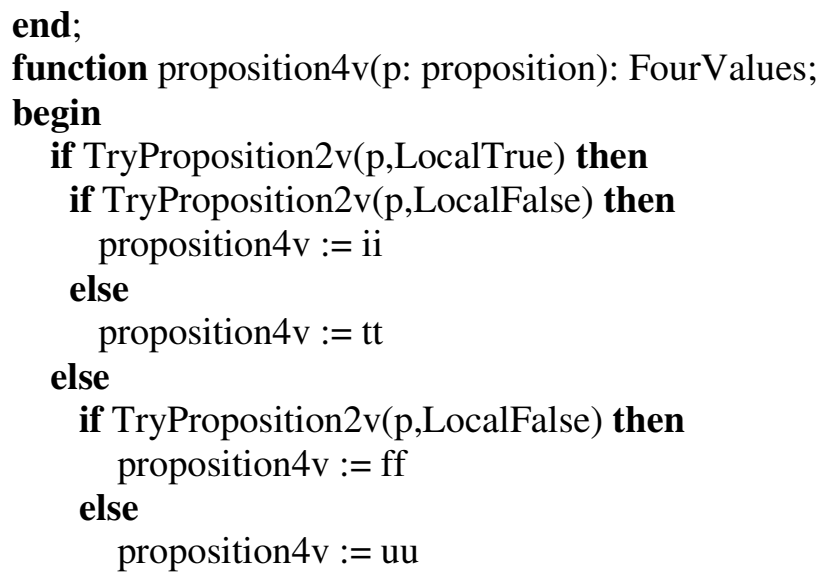

end;

Clearly, such an algorithm captures all possibilities, and can be adapted to extend from the propositional logic to a more sophisticated and even second-order logic with predicates.

Certainly, we are unable to state all mathematical true, for mathematics is a science and, as such, new theorems and proofs, new mathematical objects, are being formulated all the time by researchers. So, a proper four-valued formal system is able to state when a proposition is still unknown due to the $u u$ value. On the other hand, such a formal system captures the notion of paradoxes due to the $i i$ value. Therefore, it is sound and complete.

\section{A Four-VAlued Prolog}

For further work, a four-valued Prolog can be formally defined, implemented and used. This section introduces a brief, informal and intuitive description of the adaptation of the three-valued Prolog defined by the present author in [8]. Let us call Prolog $4 \mathrm{v}$ the sample programming language whose interpreter is intended to be the four-valued formal system.

\subsection{Syntactical and Semantic Definitions}

Definition 1. A program in Prolog4v is a sequence $S$ of clauses $c_{1} \ldots c_{n}$. Thus, it is said that a computation by $S$ proves a goal $g$ if and only if there exists some $c_{i}$ in $S$ such that $g$ is an immediate consequence of $c_{i}$, assuming that the body of $c_{i}$ can be proven. The notion of clause and body are in the following definition subsection.

Given $S$ as a sequence of clauses $c_{1} \ldots c_{n}$, a program in Prolog4v corresponds to the disjunction among all clauses. That is: $c_{1} \vee \ldots \vee c_{n}$, where the disjunctive operator $\vee$ is the same operator of the four-valued logic in table 1 . Nonetheless, the interpreter, also called formal system here, carries out its computation "downwards", i.e. from the first to the last clause. The sequence of clauses is often written like a Prolog program is, i.e. one clause fills one line.

Definition 2. A clause is a language construct which has one of the forms bellow:

or

$$
[\operatorname{not}] p\left(t_{1},, \ldots, t_{n}\right) \text {. }
$$

$$
[\mathbf{n o t}] p\left(t_{l}, \ldots, t_{n}\right) \leftarrow[\mathbf{n o t}] p_{I}\left(t_{l, l}, \ldots, t_{r, 1}\right), \ldots,[\operatorname{not}] p_{m}\left(t_{l, m}, \ldots, t_{s, m}\right) .
$$


The first clause above corresponds to a fact whereas the second clause corresponds to a rule. For instance,

$$
\text { not astar(moon). }
$$

is a fact (the moon is not a star), whereas

$$
\operatorname{shines}(\mathrm{X}):-\operatorname{astar}(\mathrm{X})
$$

is a rule (if $\mathrm{X}$ is a star, $\mathrm{X}$ shines). If one tries to prove shines(moon), the corresponding query results in $f f$, the false value.

All clauses end with a dot symbol. As usual in syntax definitions, the above brackets are not part of the language but, instead, they mean that the negation operator is optional in the clauses. Any rule contains its head, which is on the left of the inference operator $\leftarrow$, and its body, which is on the right of the same operator. At the lexical level of Prolog $4 \mathrm{v}$, there are two different inference operators to be chosen by the programmer, either ":-" like in Prolog or ":=". The former operator obeys the Closed World Assumption[9] and makes use of the Negation as Failure[10]. This means that if the body of a rule results in $u u$, the ":-" operator makes the head of the same rule become $f f$. Similarly, if the body of a rule results in $i i$, the ":-" operator also makes the head of the same rule become $f f$. The latter operator ":=" is a contribution of the present author, which obeys what he called the Open World Assumption in his PhD thesis[8] and it corresponds to the $\rightarrow$ operator described in table 1.

Briefly, if no clause unifies some given goal $g$, the answer of the query for $g$ is $u u$, the unknown constant of Prolog4v.

The body of any rule is formed by a sequence of predicates with zero or more parameters $t$ (showed with the indexes above), separated by the comma symbol (“,”), which in its turn corresponds to the $\wedge$ operator of the four-valued logic that was showed in table 1 , in the introductory section. During the computation, each predicate $p_{j}\left(t_{l, j}, \ldots, t_{u, j}\right)$ corresponds to a new four-valued goal and to a new four-valued query.

Definition 3. There exist four predefined constants in Prolog4v, namely, $f f, t t, u u$ and $i$.

The above constants correspond to the four operands F, T, U and I, respectively, of the fourvalued logic described in table 1.

Note that, in accordance with table 1, if any of those queries in a body results in $f f$, the computation of the whole rule results in $f f$ regardless of the existence of any possible inconsistency or lack of information in the other queries of the body of the rule in question. The queries are performed from left to right like in Prolog, but it is easy to see that the Prolog4v interpreter can be designed to carry out the computation in parallel and it can even distribute the computation among a number of machines. Also from table 1, note that, for any rule, the computation of the rule results in $t t$ if and only if all containing queries in its body result in $t t$, that is, the trivial and Boolean cases clearly must hold.

With respect to the ":=" inference operator, it corresponds to the $\rightarrow$ implication operator of the introduced four-valued logic, but containing the sides of the implication swapped. One could 
have chosen any pairs of operands of the $\rightarrow$ table whose results are all $t t$. However, the main diagonal of the $\rightarrow$ table is what makes sense in the real world, hence they are the choices. That is to say, $f f \rightarrow f f, \quad t t \rightarrow t t, u u \rightarrow u u$, as well as $i i \rightarrow i i$ all result in $t t$ and therefore $\rightarrow$ operator is not only sound but also makes sense in the real world. During the computation, if the body of a rule results in $i i$, the query for the whole rule results in $i i$ and, in this way, the inconsistency is propagated, possibly to the level of the user, such as a mathematician.

However, any query with the negation operator can also be treated as a unity. That is, although the four-valued logic introduced in table 1 contains the "not" operator $\ulcorner$, the system might not make use of it. Instead, the not operator can be part of the query as well as it is part of the unification algorithm, i.e. the system tries to unify the predicate including the "not" operator. Furthermore, not uu does not result in $i i$, whereas not ii does not result in $u u$ either. Instead, the system ought to propagate $u u$ and also $i i$. Thus, not uu results in uu whereas not ii results in ii. These are the only two exceptions with respect to table 1 . In other words, there are two different forms of negation.

In contrast with the negation in table 1 , let us call the not operator in the definition 2 "abstract negation". It had also been called "abstract negation" in the three-valued Prolog. Here, the negation is a four-valued extension.

Finally, the $\leftrightarrow$ operator in the above four-valued logic is simply not used by the system.

\subsection{Examples}

Consider the following example of a two-clause program in Prolog $4 \mathrm{v}$ :

$$
\begin{aligned}
& \text { happy(ann). } \\
& \text { not happy(ann). }
\end{aligned}
$$

Over the last thirty years, some proposals have been made for solving the inconsistency problem[10], such as setting priorities, possibly in some implicitly way, for all clauses. The literature on inconsistency in deductive databases and logic programs is large[12] but the present author thinks that there is little references to abstract negation.

In the above example, a query like happy(ann) clearly results in ii. Accordingly, a query like not hapy(ann) also results in $i$. In both cases, the system tries to prove both and, in accordance with the algorithm 1, it implicitly makes two binary queries, for both the positive and the negative forms of the predicate.

Now, consider the classical non-flying bird example:

$$
\begin{aligned}
& \text { fly }(X):=\operatorname{bird}(X) \text {, not penguin }(X) \text {. } \\
& \text { not } f l y(Y):=\operatorname{penguin}(Y) \text {. } \\
& \text { bird(tweety). } \\
& \text { penguin( }(Z):-\operatorname{bird}(Z) \text {, polar(Z). }
\end{aligned}
$$

To answer the query fly(tweety), the system unifies the goal with the head of the first rule, binding the variable $\mathrm{X}$ to the constant tweety. Then, the system finds the subgoal bird(tweety) 
which in turn unifies the third clause and that subquerry results in $t$. Then, in the body of the first rule, not penguin(tweety), is the next subgoal to be explored. Note that, because of the inference operator chosen, penguin is the head of a closed-world rule. Then, the subgoal unifies the fourth clause binding $\mathrm{Z}$ to tweety. As the subgoal bird(tweety) had already been proven, the next subgoal is polar(tweety). To explore this subgoal, the system does not unify any clause and, because of this, this subquery results in $u u$. The body of the fourth clause results in $u u$ since $\mathrm{T} \Lambda$ $\mathrm{U}$ results in $\mathrm{U}$ in table 1 . The subquery penguin(tweety) results in $\mathrm{ff}$ because of the closed-world assumption made by using the ":-" operator. If one replaces ":-" by ":=" in the fourth clause, the subquery penguin(tweety) results in $u и$ instead.

Now, consider a new clause

$$
\text { polar(tweety). }
$$

is asserted and that the system places it at the end of the sequence of clauses. For the same query fly(tweety), the system now answers $f f$. That is, it learns. In comparison to a similar Prolog program:

$$
\begin{aligned}
& f l y(X) \text { :- } \operatorname{bird}(X), \text { not penguin( }(X) \text {. } \\
& \operatorname{bird}(\operatorname{tweety}) . \\
& \text { penguin(Z) :- } \operatorname{bird}(Z), \operatorname{polar}(Z) \text {. }
\end{aligned}
$$

The same query fly(tweety) would have resulted in true because the third clause alone ensures that only a polar bird is a penguin. That is, until the knowledge base is complete, the system sometimes gives wrong answers with respect to the real world. For instance, for a query such as fly(airplane), the answer is $u u$ in the Prolog $4 \mathrm{v}$ program above, whereas the same query results in false in the three-clause Prolog program above. Following this, binary formal systems are clearly not appropriate to write mathematical truths.

As another example, suppose that one knows that Berne is the capital of Switzerland and that each county has one capital only. In Prolog4v, one would write

$$
\begin{gathered}
\text { capital(berne,switzerland). } \\
\text { not capital }(X, Y):=\operatorname{capital}(Z, Y), X<>\text { Z. }
\end{gathered}
$$

where $<>$ stands for the different from $(\neq)$ operator. A non-ground query, i.e. a query where there is some unbound variable, for instance capital(bern, $X$ ) (note the different spellings) would result in $\mathrm{X}=u u$, whereas a ground query such as capital(zurich, switzerland) would definitely result in $f f$ as follows: the corresponding goal would not unify the first clause but would unify the second one because the presence of the not abstract negation in its head does not fail during the computation of the unification algorithm. In this case, $\mathrm{X}$ is bound to zurich and $\mathrm{Y}$ is bound to switzerland. Then, the system tries to prove the subgoal capital(Z,switzerland) and unifies the first clause, i.e. the fact capital(berne, switzerland) binding $Z$ to berne. Now, the system evaluates the expression $\mathrm{X}<>\mathrm{Z}$, which in turn results in $t t$ as zurich is not berne. Since all premises of the rule are true, the body results in $t t$ and the system concludes that the head not capital (zurich,switzerlan) is $t t$ and, hence, that capital (zurich,switzerland) is $f f$, and that is the response of the query at the user's level. 


\section{CONCLUSiOnS}

There exists some four-valued formal system that is able to state arithmetical truths including paradoxes[13]. If it is possible for a machine to generate all truths, the same formal system can be used on a suitable knowledge base for that purpose. Queries reaching paradoxes are answered with $i i$, the inconsistent value. On the other hand, the claimed system is also able to answer queries with $u u$, the unknown value. The content of the present article was meant to be comprehensive even for undergraduate student. Philosophy students can also understand it.

However, all the work is clearly implementable on a common machine, such as Turing machine or von Neumann machine. Such a machine is binary and based on Boolean logic. An external four-valued layer is built. As a consequence, although the layer is relevant from the point of view of the visualization of the problem and the organization of the solution, it is proven that Gödel's theorem is clearly invalid.

The computation by the referred formal system roughly takes the double the time of a typical binary formal system: some time for trying to prove that a goal is true and some additional time for trying to prove that the same goal is false. Therefore, its computation is polynomial.

Further work can be the formal definition of the four-valued Prolog, including its formal syntax and semantics as well as the unification algorithm.

\section{ACKNOWLEDGEMENTS}

Many thanks to Alan Turing for his machine.

\section{REFERENCES}

[1] Epstein, Richard L. and Carnielli, Walter A. (2000) Computability: computable functions, logic, and the foundations of mathematics, Wadsworth, an International Thomson Publishing Company, second edition.

[2] Gödel, Kurt, (1931) Über formal unentscheidbareSätze der Principia Mathematica und verwandterSysteme I. MonatsheftefürMathematikunPhysik, Vol. 38, pp173-198.

[3] Ferreira, Ulisses (2000) uu for programming languages. ACM SIGPLAN Notices, 35(8): pp20-30.

[4] Ferreira, Ulisses (2004) A five-valued logic and a system. Journal of Computer Science and Technology, 4(3): pp134-140, October.

[5] Ferreira, Ulisses (2004) Uncertainty and a 7-valued logic. In Pradip Peter Dey, Mohammad N. Amin, and Thomas M. Gatton, editors, Proceedings of The 2nd International Conference on Computer Science and its Applications, pp 170-173, National University, San Diego, CA, USA, June.

[6] Belnap Jr, Nuel, (1975) A useful four-valued logic. In J. Michael Dunn and George Epstein, editors, Proceedings of the Fifth International Symposium on Multiple-Valued Logic, Modern Uses of Multiple-Valued Logic, pp 8-37. Indiana University, D. Reidel Publishing Company. 
[7] Bratko, Ivan (1986) Prolog programming for Artificial Intelligence, Addison-Wesley Publishing Company.

[8] Ferreira, Ulisses (2004) A prolog-like language for the internet. In Veljko Milutinovic, editor, Proceedings of IPSI CAITA-04, Purdue, Indiana, USA.

[9] Reiter, R. (1978) On Closed World Data Bases in Logic and Data Bases, Plenum Press, New York, pp $55-76$.

[10] Clark, Keith (1978) Negation as Failure in Logic and Data Bases, Plenum Press, New York, pp 293322.

[11] Dung, P. M. \& Mancarella P. (1996) Production Systems need Negation as Failure, Proceedings of the XIII National Conference on Artificial Intelligence, vol 2, AAAI Press and the MIT Press, pp 1242-1247.

[12] Seipel, Dietmar (1998) Partial Evidential Stable Models for Disjunctive Deductive Databases, in Logic Programming and Knowledge Representation, Third International Workshop / LPKR'97, Lecture Notes in Artificial Intelligence, vol. 1471, Springer, New York, October, pp 66-83

[13] Ferreira, J. Ulisses (2017) A Note on Gödel's Theorem, International Journal of Computer Science and Information Technology, Vol. 9, No. 2, pp 69-76.

\section{AUTHOR}

The present author studied as a Master student at the Universidade Federal da Paraíba in Campina Grande, Brazil, and as a postgraduate student in the Department of Computer Science at the University of Edinburgh, and did some further research work at Trinity College in Dublin from 1998 until 2001.

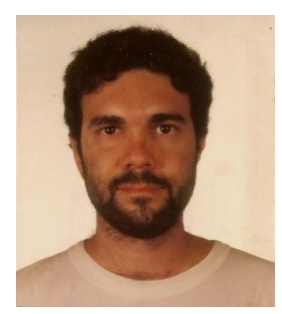




\title{
SCALABLE AND EFFICIENT PATH- SENSITIVE ANALYSIS TECHNIQUE SCANNING MANY TYPES OF VULNERABILITY
}

\author{
Dongok Kang and Minsik Jin* \\ PA Division, Fasoo.com R\&D Center, Seoul, Repulic of Korea
}

\begin{abstract}
The goal of this paper is to present an efficient and effective path-sensitive analysis technique for many types of security vulnerability. We propose two analysis techniques. The first is a scalable path-sensitive analysis technique for security vulnerability with high precision and recall. Our strategies are to allow flexible design of path state and to make an effective path navigation heuristic which achieves both scalability and high recall. Experimental results show that a vulnerability scanner implemented through this technique get precision $100 \%$ and recall 93\% on OWASP Benchmark. The vulnerability scanner is able to analyze 1 million lines of code. The second is a pre-analysis technique to improve the efficiency of the above analysis technique. The pre-analysis technique improves the path navigation by using an additional cheap anlysis. Despite the additional cost, experimental results show that the total analysis time is reduced by 2.5 times. Simultaneously recall of the analysis is improved by the pre-analysis technique.
\end{abstract}

\section{KEYWORDS}

Secure coding, Security, Static analysis, Vulnerability scanner, Summary-based, Path-sensitive, Information flow Analysis, Pre-analysis

\section{INTRODUCTION}

The goal of this paper is to present an efficient and effective path-sensitive taint analysis technique scanning many types of security vulnerability at once. First, we propose an effective path-sensitive analysis technique to achieve high precision and recall. Precision is the ratio of the number of true positives to the number of detected vulnerabilities. Recall is the ratio of the number of true positives to the number of all vulnerabilities. For practical analysis, path-sensitive analysis techniques make use of abstractions of partial paths or symbolic values and path navigation heuristics. Our technique does not care about abstraction to give flexibility to the design of path state. Our technique is easy to achieve high precision because of this feature. It optimizes only path navigation. Therefore path navigation should avoid exploring non-vulnerable paths as much as possible for scalable analysis. On the other hand, path navigation should explore all vulnerable paths as much as possible to achieve high recall. We propose a path navigation

Natarajan Meghanathan et al. (Eds) : ACSIT, SIPM, FCST, CoNeCo, CMIT - 2017

pp. 21- 40, 2017. (C) CS \& IT-CSCP 2017

DOI : $10.5121 /$ csit.2017.70803 
strategy satisfying these goals. We evaluate its ability to achieve high precision and recall on OWASP Benchmark. Second, we propose a pre-analysis technique making the path-sensitive analysis several times more efficient. It improves the efficiency of a baseline analysis preserving the original precision and recall. We evaluate the improvement of efficiency on 12 real-world applications. In summary, our goal is

1. Design a scalable path-sensitive analysis technique to scan many types of security vulnerability with high precision and recall.

2. Design a pre-analysis technique to improve the efficiency of the path-sensitive analysis not sacrificing the original precision and recall.

Inter-procedural path-sensitive analysis scanning security vulnerabilities suffers from path explosion problem. The number of paths is exponential to the number of nodes in a control flow graph. Including loop, it may be infinite. All vulnerabilities can be detected by analyzing all the paths, but it is impossible to analyze all of them.

Generally, there are two ways to overcome the path explosion problem in path-sensitive analysis. One way is to analyze each query one by one collecting predicates to be satisfied backward from a query point in a fully path-sensitive manner. It is practical for analyzing small size of queries and has an advantage in precision. A disadvantage is that it is difficult to analyze complex data types and operations. The other way is forward analysis with abstractions of partial paths or values that are irrelevant to interesting properties and with path navigation heuristics selecting paths related to interesting properties.

Forward analysis with path navigation is more viable option for many types of security vulnerability scanner than backward analysis. The size of query points for checking security vulnerabilities is usually large. Just XSS only analysis has too many query points such as JSP's out.write()s. There are several types of security vulnerability such as Command Injection, SQL Injection, Path Traversal, etc. Even if each of them has small size of query points, the sum of them may be large. Backward path-sensitive analysis is not appropriate for scanning many types of security vulnerability because they may have large size of query points. In contrast, forward analysis has an advantage in analyzing many queries at once. We present a path-sensitive analysis technique for security vulnerability that uses forward analysis in Section 3.

However, forward analysis involves a risk to analyze paths that are irrelevant to vulnerabilities. Figure 1 is a motivating example. There are 2 taint sources and 2 sinks. Method SOURCES returns a T1-type tainted value in $\{1\}$ and a T2-type tainted value in $\{2\}$. Method SINKS propagate parameter $S$ to the $\mathrm{T} 1$-type sink in $\{3\}$ and the T3-type sink in $\{4\}$. Method PROP has no taint sources or sinks. It propagates a tainted value in parameter $X$ to the $P 1$ in $\{5\}$ and the $P 2$ in $\{6\}$. Method FOO just calls them sequentially: SOURCES, PROP, and then SINKS. A T 1type vulnerability may exist through the path $\{1\}\{4\}\{5\}$. There are no other vulnerabilities. The most efficient way to scan the vulnerability is making only three path summaries: (c1, \{return $\mapsto$ $\mathrm{t} 1\{1\}\}),(\neg \mathrm{c} 2,\{\mathrm{p} 2 . \mathrm{str} \mapsto \mathrm{p} 2 . \mathrm{str}+\mathrm{x}\}),(\mathrm{c} 3,\{\mathrm{t} 1 \operatorname{sink}\{6\} \mapsto \mathrm{s}\})$. By instantiating them, only one path summary is calculated for method FOO: $(\mathrm{c} 1 \wedge \neg \mathrm{c} 2 \wedge \mathrm{c} 3, \mathrm{t} 1 \sin k\{6\} \mapsto t 1\{1\})$. However, there are no inducements to navigate only branch $\{1\}$ in SOURCES, only branch $\{4\}$ in PROP, and only branch $\{5\}$ in SINKS. A path-sensitive analysis has no choice but to result in 8 path summaries for FOO. Despite the time is wasted to analyze non-vulnerable paths, it is difficult to make the path navigation efficient. 
We propose a pre-analysis technique for efficient path navigation. The overall process is as follows. A pre-analysis provides program points that may propagate tainted values to their sink points. In Figure 1, it may provide $\{1\},\{4\}$, and $\{5\}$. And then the target path-sensitive analysis utilizes the program points for selecting branches to navigate. The target analysis navigates only branches $\{1\},\{4\}$, and $\{5\}$. In this manner, the prior knowledge provided by the pre-analysis makes aggressive path navigation possible in the target analysis.

A challenging point is that the additional time for the pre-analysis should be less than the reduced time of the target analysis. A poor pre-analysis may give the target analysis invaluable knowledges, for example, all branches in Figure 1. The pre-analysis is not useful to reduce the analysis time of the target analysis. On the other hand, if a pre-analysis spends too much time for valuable knowledge, it is also meaningless work.

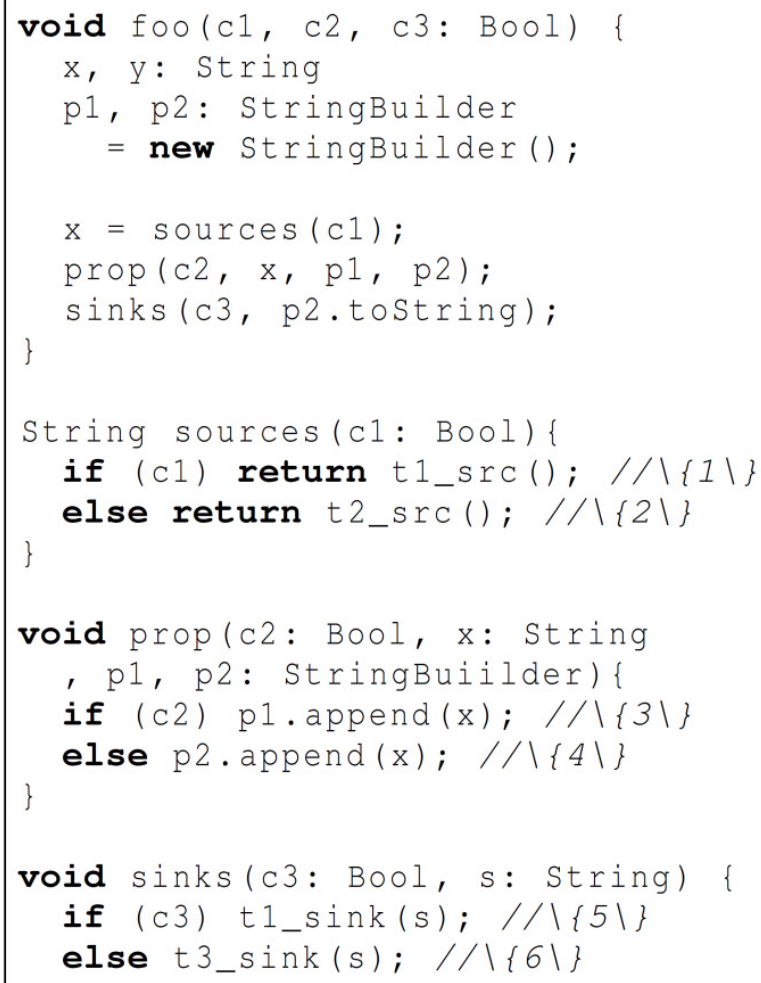

Figure 1: Only exploring $\{1\},\{4\}$, and $\{5\}$ is enough to make a summary of method foo

The pre-analysis technique involves a risk that a selected branch may block exploring another selected branch. Pre-analysis considering only propagations of tainted values do not give a knowledge of feasible paths. Not only points related to tainted values but also points related to calculation of branch conditions should be considered for path navigation. Figure 2 shows an example of the problem. ZIPFILE contains a tainted value and the sink is in PROCESSFILE of line 9. It is impossible to execute code at line 9 without passing code at line 6 . Method PROCESSPROPERTYFILE contains two branches and each of them returns different boolean 
value. For exploring line 9, PROCESSED at line 8 should have false value. Therefore a path navigation should explore the false branch in PROCESSPROPERTYFILE.

We solve the problem by marking may-feasible partial paths in advance. Using heuristic methods, the pre-analysis finds intervals that contain pairs of a definition of a variable and a branching condition using the variable. We call the intervals as effective intervals. In Figure 2, ([line 3], [line 9]) is an effective interval. The pre-analysis calculates infeasible partial paths in the intervals by path-sensitive constant propagation. And then the other paths in the intervals are marked as may-feasible paths. In Figure 2, [line 3][line 6][processPropertyFile; line 22][line 9] is a mayfeasible path in the effective interval ([line 3], [line 9]). The target analysis utilizes the knowledge to avoid infeasible paths. We discuss the reason why this strategy, constant propagation within small code interval, effectively solves the problem in Section 2.

This paper is organized as follows. Section 2 introduces previous researches about path-sensitive analysis. Section 3 introduces a scalable and effective path-sensitive analysis technique. Section 4 explains about a pre-analysis technique to make the path-sensitive analysis more efficient. Section 5 explains experimental results and discuss about pros and cons of our analysis techniques.

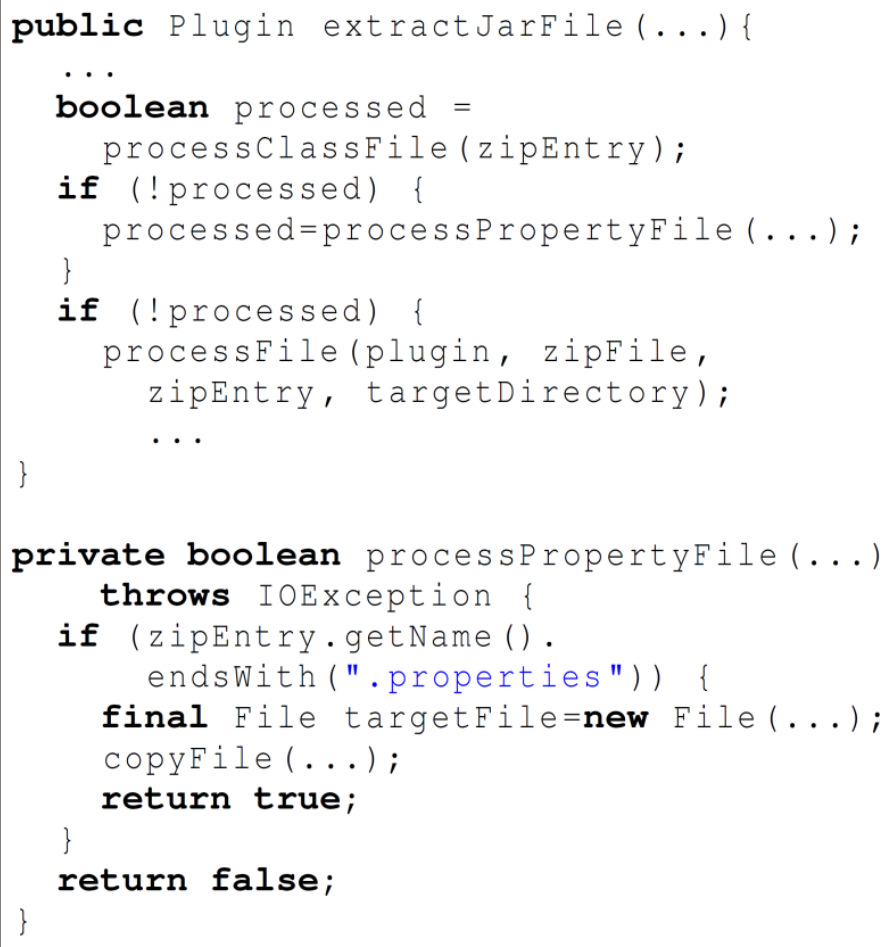

Figure 2: An example code from PluginExtractor.java of Webgoat. Path navigation of line 9 is required for detecting a vulnerability. It is possible only when the analysis explore the false branch of processPropertyFile. 


\section{RELATED WORK}

In this section we survey previous approaches to path-sensitive analysis. Path-sensitive analysis technique was used for various purposes such as points-to analysis, security vulnerability scanner, memory leak detection, compiler optimization, and language refinement. They fall into two categories: backward analysis and forward analysis. Backward analysis techniques are used for one special property. Each analysis technique includes optimization methods to overcome path explosion problem. The optimizations are performed by ignoring or abstracting paths and values unrelated to the properties of interests.

[9] avoids unnecessarily explored path by join that do not hurt accuracy. The work is to find infeasible paths for compiler optimization. They formalize edge strings and discuss optimizations. They explain a concept of delayed join. The method uses k-edge abstraction inspired by k-context abstraction. They insist 2-sensitive edge strings are enough for finding infeasible paths. This fact inspired us to use a heuristic using small size of codes for finding infeasible paths. Although it is difficult to make use of this work as it is, it shows that there are localities between definitions of a variable and the uses of the variables in branch conditions.

[11] presents an optimization technique abstracting away certain symbolic subterms to make the analysis practical. By abstracting elements unrelated to a property of interest, it reduces analysis time. They test only small codes to show the efficiency.

[8] presents a vulnerability detection technique using a backward analysis. Program condition(PC) and security condition(SC) are constructed to prove a safety of a query point. It is proved by solving PC $\wedge \overline{S C}$. The weak point of this work is that it does not handle complex data types because of the limitation of backward analysis.

[7] presents a demand-driven analysis technique for buffer overflow detector. The technique is backward analysis for buffer access violation. It is the efficient way to analyze the property. The method makes use of another path-insensitive pointer analysis. The requirement of timeconsuming pointer analysis is a limitation of this work.

[1] solves infeasible paths problem by abstract interpretation. The method refines syntactic language for another path-sensitive analysis. It gives us a lesson that removal of infeasible path is important to prove more queries. It only concentrates on improvement of true positive rate. The cost of infeasible-path detection increases the total analysis time.

[3] presents a technique merging branches unrelated to properties of interest. It gives us a lesson that avoiding unrelated branch is important to make analysis efficient. Proposed method is difficult to be used as it is for making vulnerability scanner because a vulnerability scanner has to consider sanitizer.

[15] presents a technique to detect RCE vulnerability. The method slices codes related to sinks and checks the satisfiability of a sink's collected predicates.

[12] uses a global invariant approach to detect data race avoiding the path explosion problem. They do not present an experimental result that shows scalability to real-world programs. 
[5] shows a method to add path sensitivity to points-to analysis. Their approach works with both WPP2G and P2SSA representations yielding a certain degree of path-sensitivity. It is a technique abstracting elements unrelated to the property of interest.

[6] presents a backward slicing technique that excludes spurious dependencies lying on infeasible paths and avoids imprecise join. It traverse a program in a depth-first search manner and reuse dependencies from precomputed paths. The novelty of this work is a formalization of efficiently reusing conditions. Experimental results of the prototype does not show scalability.

[14] presents a scalable path-sensitive analysis for memory leak detection. It is a great work that concentrates on memory leak detection. They presents efficient summarizing method for summaries to get scalability.

[13] presents an optimized backward analysis technique for data flow analysis. They optimize the path-sensitive analysis by abstract subterms as unknown values in a predicate. They present a calculation of predicates containing unknown terms.

[4] presents a formal method for computing the precise necessary and sufficient conditions for program properties that are fully context- and path-sensitive. They show a sound, complete and scalable analysis for only small functional language. Experimental results of the prototype on real-world programs does not show high precision.

\section{PATh-SENSITIVe ANALYSIS For SECURITY VULNERABILITY}

The target program is a list of bodies composed of a list of statements and a control flow graph. Figure 3 shows the target program. pgm means a program. It is list of bodies with method name $\left(\left(\right.\right.$ body $\left.\left._{\mathrm{f}}\right)+\right)$. A body consists of a list of statements and a control flow graph. The statements consist of assignment statement $(\mathrm{x}:=\mathrm{e})$, assert statement $(\operatorname{assert}(\mathrm{x} \odot \mathrm{e}))$, and call statement $(\operatorname{call}(\mathrm{f}, \mathrm{x}))$. Expression $\mathrm{e}$ is composed of typical expressions such as arithmetic expressions or logical expressions, l-value lv, and primitive values primitive.

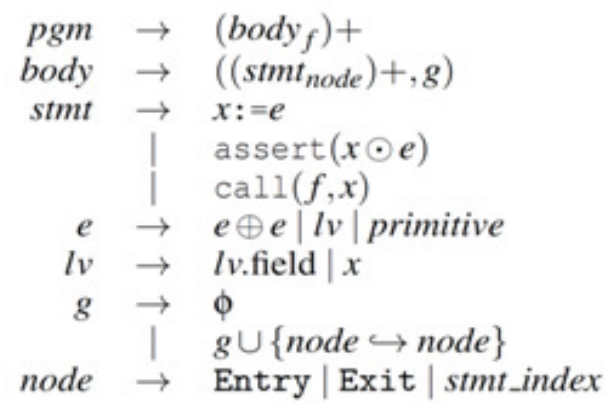

Figure 3: A program contains statements and control flow graphs

Our path-sensitive analysis technique uses summary-based analysis technique. A summary-based analysis analyzes each method in bottom-up call order generating a summary consists of symbolic states. Summaries are instantiated in each call statement making use of each caller's context. Summary-based analysis is a typical choice for path-sensitive analysis because of scalability. Figure 4 shows the summary design of the analysis. Summary describes the 
summary. A summary is a set of path summaries. A path summary is a symbolic state of a path. A path summary consists of a path string Path, last node's symbolic memory SMemory, and each symbolic value's constraints SConstraint. We do not present the specific designs of SConstraint and SMemory because our path-sensitive analysis technique does not care about the complexity of the summary design. Our goal is to get scalability not by a low-cost summary but by a path navigation strategy.

$$
\begin{aligned}
\text { Summary } & =\wp(\text { PathSummary }) \\
\text { PathSummary } & =\text { Path } \times \text { SConstraint } \times \text { SMemory } \\
\text { Path } & =(\text { Method } \times \text { Node }) \text { List } \\
\text { SConstraint } & =\text { SVal } \rightarrow \text { Constraint } \\
\text { SMemory } & =\text { SRef } \rightarrow \text { SVal }
\end{aligned}
$$

Figure 4: Summary is a set of path states

Figure 5 shows the semantics of our analysis technique. It calculates output path summaries from an input path-summary. next(f, $i)$ is successor nodes of a node (f, i) in the control flow graph. An assignment statement changes the state of symbolic memory by storing evaluated symbolic value $[[\mathrm{e}]] \mathrm{m}$ to the evaluated location $[[\mathrm{x}]]_{\mathrm{lv}} \mathrm{m}$. An assert statement changes the state of symbolic constraint by storing the evaluated constraint. If an evaluated constraint is proved to be false, the path summary is discarded. The analysis propagates summaries of each method bottom-up through the call order. At a call statement, it lookups the callee's precomputed summaries $\operatorname{SUM}\left(\mathrm{f}^{\prime}\right)$ and instantiate it in the caller's context. In this way, it inter-procedurally detects vulnerable paths.

We introduce the details of our path sensitive analysis technique. The analysis explores local path in a depth-first search manner and filters most likely to be vulnerable paths among several interprocedural paths. Algorithm 1 describes the details. It calculates path summaries visiting local nodes. WEIGHTPATHFILTER is a heuristic algorithm for filtering path summaries that are most likely to be vulnerable. It is applied to the intermediate path summaries for each iteration. At a call statement of a local path, there may be n inter-procedural paths with each symbolic state and $\mathrm{m}$ path summaries. After instantiation, there are, at most, $\mathrm{n} \times \mathrm{m}$ inter-procedural paths with each symbolic state. At this point, WEIGHTPATHFILTER reduces the size of path summaries. The path navigation halts when $100 \%$ of node coverage is achieved or iteration is over the threshold.

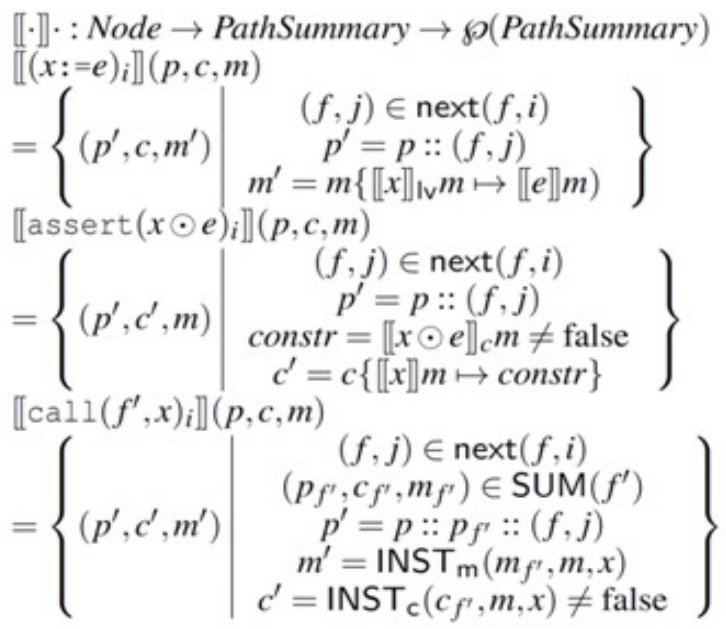

Figure 5: Analysis semantics 


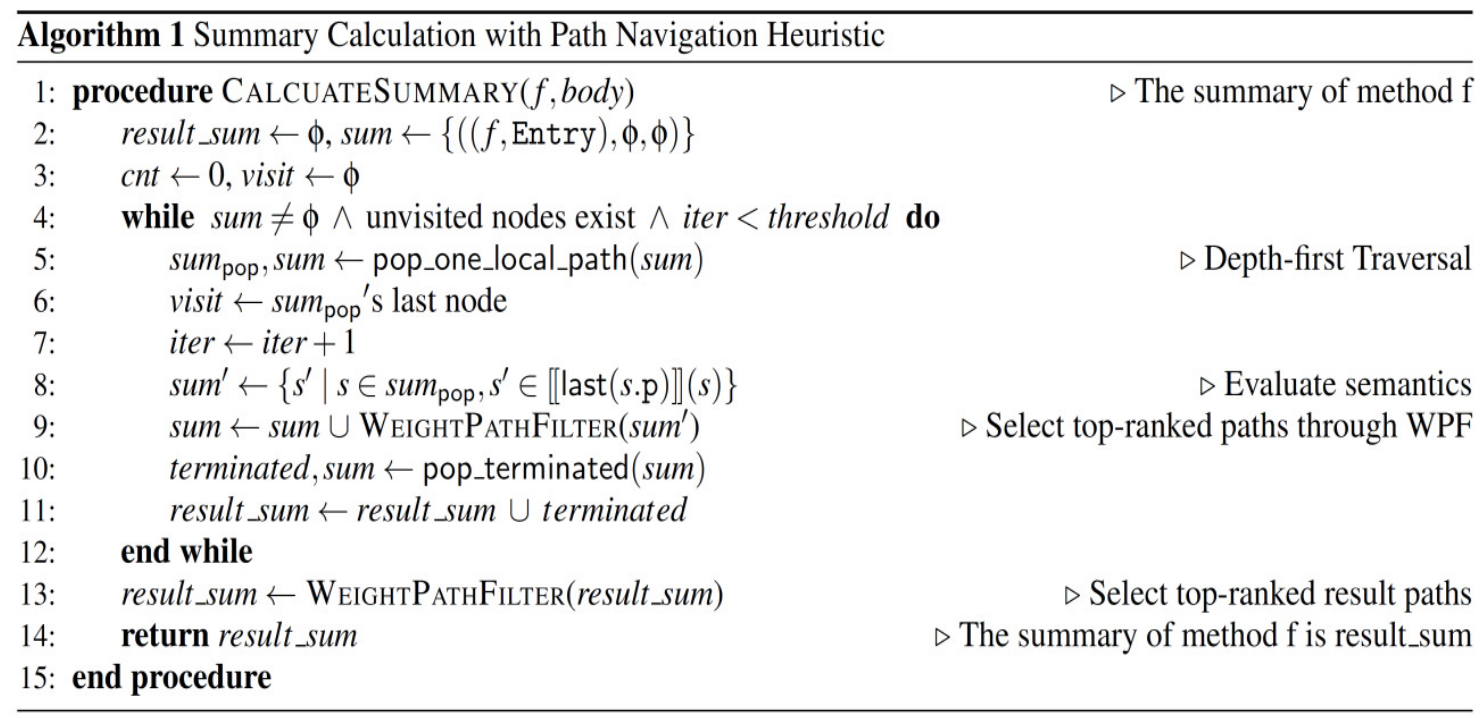

WeIGHTPATHFILTER algorithm is described on Algorithm 2. It simply sorts path summaries according to their respective scores and picks the top-ranked path summaries. The sorting criteria $W$ contains several heuristics. Mainly, path summaries with following properties are preferred.

- many tainted values and sinks.

- many parameter symbols.

- short path.

- many equal constraints.

Path summaries having more parameter symbols are preferred than others because it has higher possibility of propagating tainted values. Path summaries having shorter paths are preferred than others because most of vulnerabilities have short vulnerable paths. Equal constraints are useful for pruning infeasible paths, therefore it is preferred.

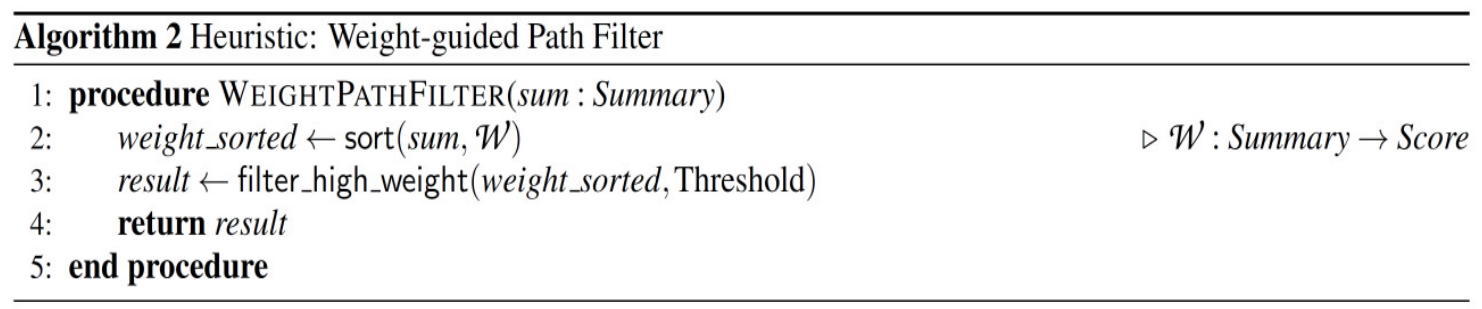

The introduced path-sensitive analysis technique is scalable and has an ability to achieve high precision and recall. We evaluate the performance in Section 5.

\section{PRE-ANALYSIS TEChNiQUe}

We present a pre-analysis technique to improve efficiency of a target path-sensitive analysis. In Section 1, we address an inefficiency of path-sensitive analysis which is difficult to be improved. To avoid path navigation of non-vulnerable paths, we propose a pre-analysis technique providing 
prior-knowledge for target path-sensitive analysis. We call the prior knowledge as Path-oracle, the pre-analysis as Pre-analysis, and the target path-sensitive analysis as Main-analysis.

\subsection{Path-oracle}

Here, we describe Path-oracle provided to Main-analysis by Pre-analysis. Figure 6 shows the Path-oracle description. Path-oracle consists of three part: Pick, San, and Allowed. Pick is a set of nodes that may be included in vulnerable paths. San is a set of nodes that may sanitize tainted values. Allowed is a set of may-feasible partial paths. The form of Allowed is a map from a starting node to set of may-feasible partial paths. Allowed is used to avoid infeasible paths by reserving the paths in each path summary

$$
\begin{aligned}
& O \in \text { PathOracle }=\text { InfoFlowHint } \\
& \times \text { SanitizingHint } \\
& \times \text { AllowedPaths } \\
& \text { Pick } \in \text { InfoFlowHint }=\text { Node } \rightarrow \text { Bool } \\
& \text { San } \in \text { SanitizingHint }=\text { Node } \rightarrow \text { Bool } \\
& \text { Allowed } \in \text { AllowedPaths }=\text { Node } \rightarrow \\
& \wp(\text { ReservedPath })
\end{aligned}
$$

Figure 6: Path Oracle

\subsection{Challenge of Pre-analysis}

A Pre-analysis should achieve the following goals to be useful.

- Lightweight analysis

- Conservative detection of vulnerabilities

- Accurate enough to provide useful Path-oracle.

A Pre-analysis should occupy little portion of total analysis time. Our goal of the pre-analysis technique is reducing the total analysis time by improving the efficiency of a Main-analysis. If the analysis time of a Pre-analysis is longer than the reduced time of the Main-analysis, it is useless.

A Main-analysis makes use of a Path-oracle aggressively to its path navigation, therefore it should detect all vulnerable paths as possible. A Main-analysis tries to visit only nodes in Pick. Therefore, the result of a Pre-analysis should include all possible nodes included in vulnerable paths.

Too imprecise Pre-analysis result misleads the Main-analysis into wasting time. A Main-analysis tries to cover all nodes in Pick. Unnecessarily selected nodes in a Pre-analysis lead to unnecessary path navigations in the Main-analysis. A Pre-analysis should provide essential nodes only.

\subsection{Pre-analysis Memory Model}

A Pre-analysis makes use of abstract interpretation[2]. A Pre-analysis is context/flow-insensitive and field-insensitive. It has only one memory state $d \in D$ for all program points. Figure 7 shows the design. The abstract location of memory state is variable. Each field strings are ignored. 
A Pre-analysis collects vulnerable variables and nodes. Source map $\in \operatorname{Src}$ and sink map $\in$ Sink consist of a map from variable to related taint rules and a map from node to related taint rules. The variable map is for analyzing flow of taint rules. The node map is for producing Path-oracle. A Pre-analysis collects only nodes for sanitizer, because it is not interested in sanitizing flows.

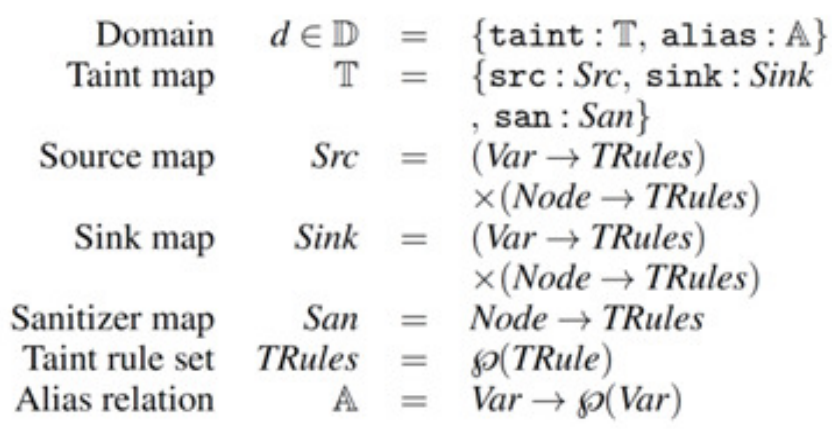

Figure 7: Memory model of Pre-analysis

\subsection{Pre-analysis Semantics}

Figure 8 shows the semantics on the memory model described in Figure 7. It describes transition rules of $\mathrm{D}$ for each statement. Notice that it does backward propagation for detecting the flows to a sink. And it does forward propagation for detecting the flows from a source. It collects alias information to detect taint propagation by heap location.

Figure 9 describes field-insensitive semantics about l-value evaluation. $[[\operatorname{lv} . f]]_{l v} d=[[\operatorname{lv}]]_{l v} d$ presents that we do not care about field. $[[x]]_{l v} d=\{x\} \cup$ d.alias $(x)$ presents that it considers alias while evaluating l-value.

\subsection{Pre-analysis Algorithm}

A Pre-analysis calculates approximate fixed point of D. In Algorithm 3, codes at line 5-10 calculate it. During the iteration, taint rules of sources and sinks are propagated through the semantics. For efficiency, the while loop halts when its iteration exceeds threshold. It halts when d is saturated through the semantics.

A Pick is made with Src and Sink of D. A Pre-analysis intersects each node's taint rules in Src and Sink to find vulnerable nodes. The initial Pick is made of the intersected taint rules. Code at line 13 in Algorithm 3 then propagates each taint rules of the nodes in the Pick to its dominator nodes. This process is described in Figure 10 (a), (b). Before line 13, it looks like (a). Propagation nodes of sources and sinks are only picked. After line 13, it looks like (b). A path related to the vulnerability is constructed naturally. 


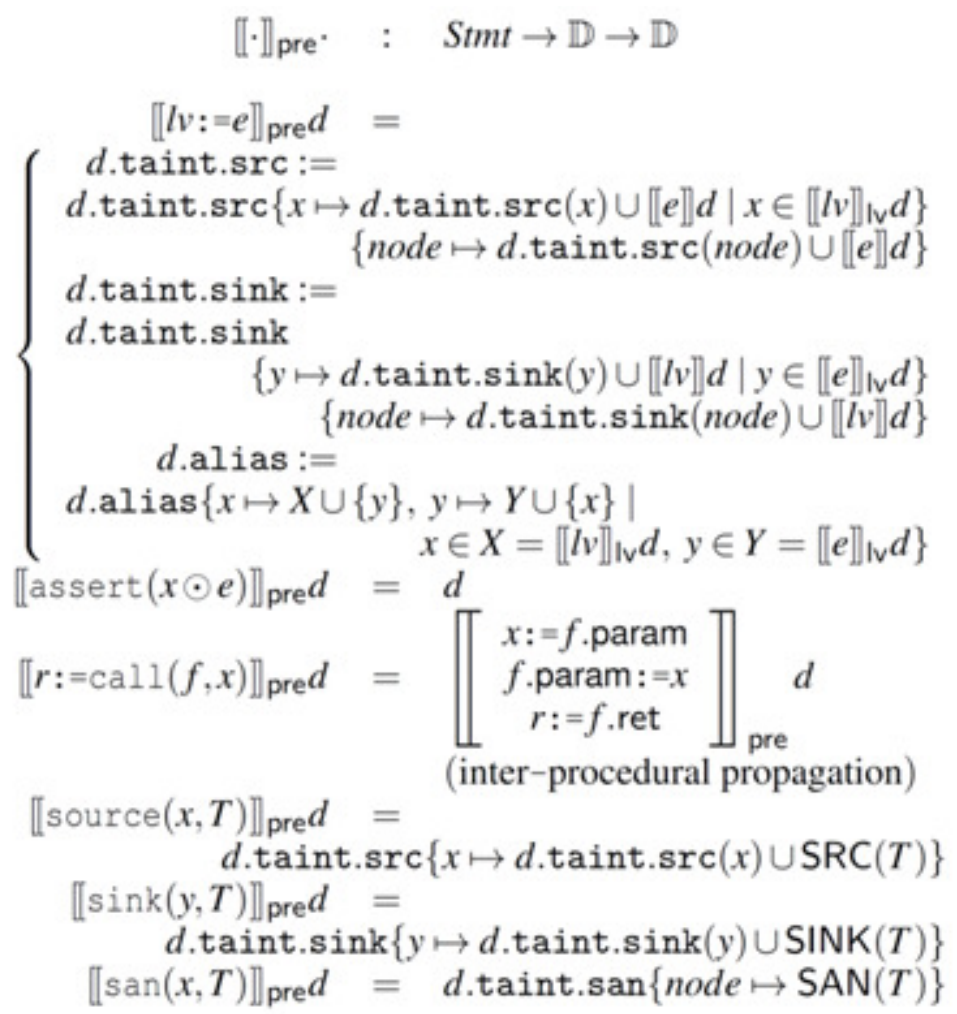

Figure 8: Semantics of Pre-analysis

$$
\begin{aligned}
& \llbracket \cdot \rrbracket_{\text {IV }} \cdot \quad: \quad \text { Expr } \rightarrow \mathbb{D} \rightarrow \wp(\text { Var }) \\
& \llbracket e \oplus e \rrbracket_{\mathrm{IV}} d=\llbracket e \rrbracket_{\mathrm{Iv}} d \cup \llbracket e \rrbracket_{\mathrm{Iv}} d \\
& \llbracket l v \cdot f\left\|_{\mathrm{lv}} d=\llbracket l v\right\|_{\mathrm{lv}} d \\
& \llbracket x \rrbracket_{\mathrm{Iv}} d=\{x\} \cup d \text {.alias }(x)
\end{aligned}
$$

Figure 9: Evaluation rule for I-value in Pre-analysis

A San is made with San of D. Different from Pick, it only propagates each nodes to their mutually dominant nodes. A Main-analysis uses San passively in path navigation. This is why a Preanalysis propagates sanitizing nodes passively. In Figure 10 (c), the square node is sanitizing node. After line 16, it looks like (d). It changes only node $\{3\}$ as a sanitizing node. Since the Main-analysis knows $\{3\}$ is an entrance to the sanitizer, it may explore a vulnerable path $\{1\}\{2\}\{5\}\{6\}$.

An Allowed is made with constant propagation on effective_interval. In line 18 of Algorithm 3, scan_effective is an effective interval classifier. For example, see Figure 10 (e). Interval ( $\{2\}$, $\{9\})$ is an effective interval. Last node $\{9\}$ is picked as propagation node. There are many possible paths between $\{2\}$ and $\{9\}$ and two boolean value assignments exist. A Pre-analysis does path-sensitive constant propagation on this interval. The Pre-analysis may discover two path $\{2\}\{3\}\{7\}\{9\},\{2\}\{4\}\{6\}\{7\}\{9\}$ are infeasible. For finding effective interval, it uses several heuristics such as the number of boolean assignment. Low cost for finding those effective intervals is important. 


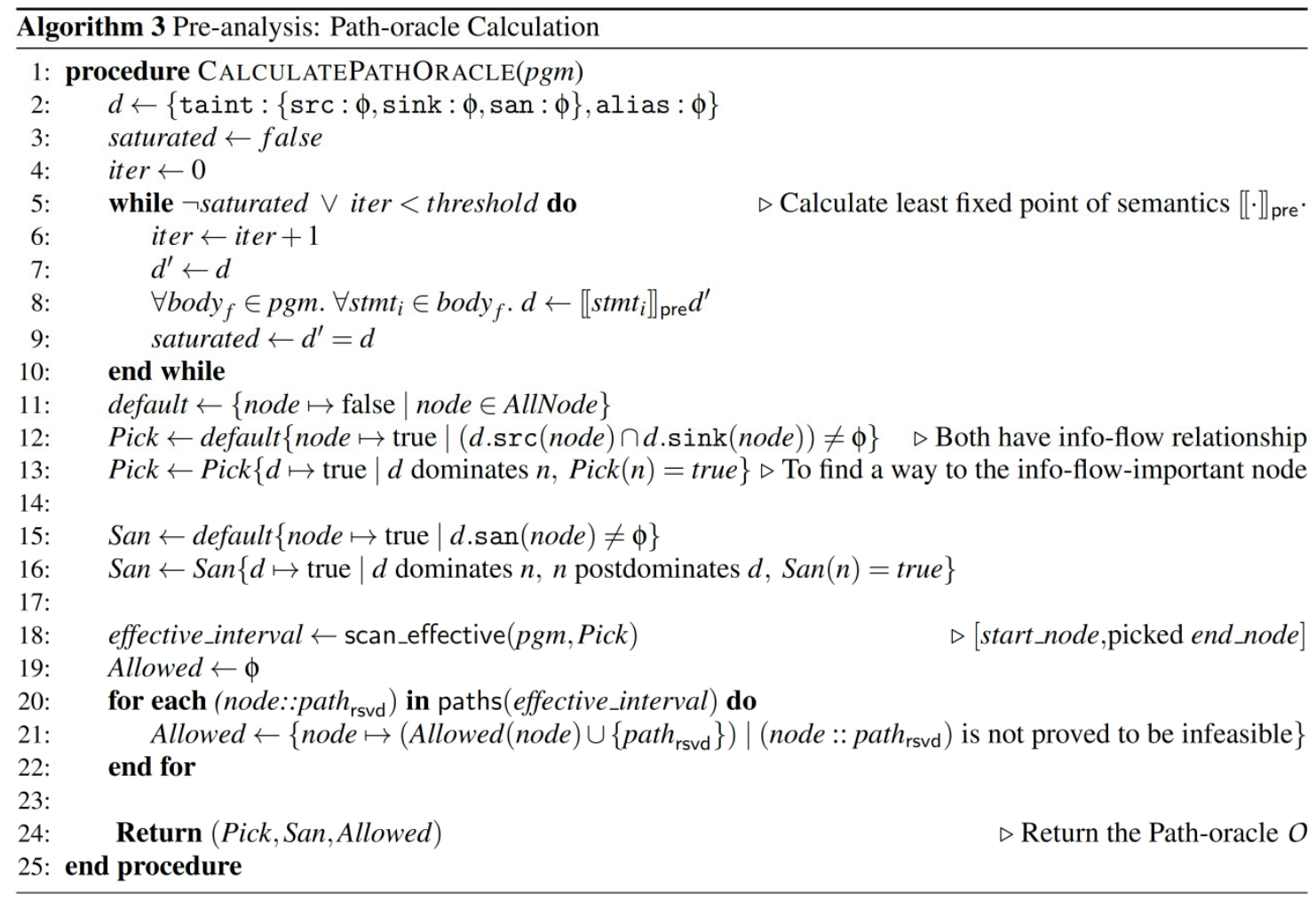

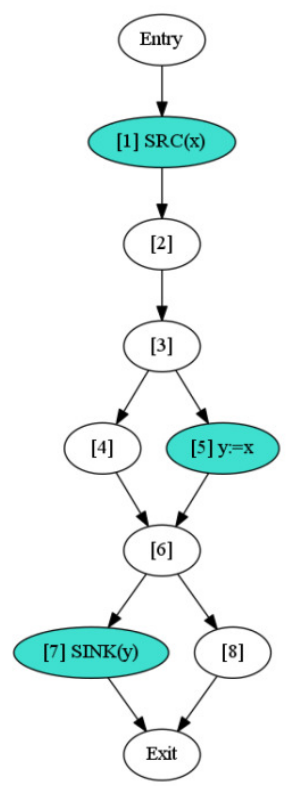

(a) Initial Pick

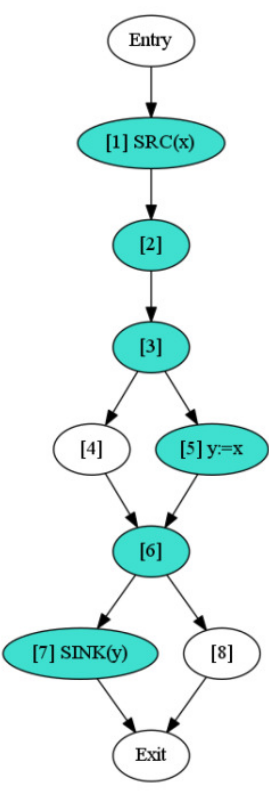

(b) Propagated Pick

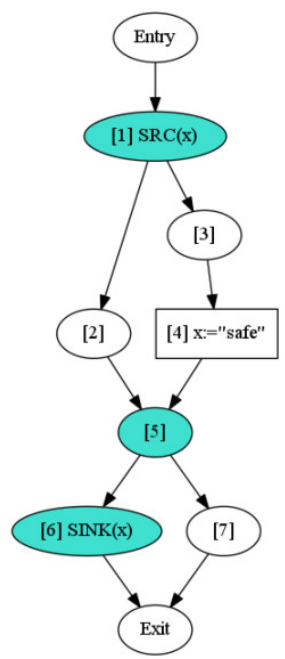

(c) Initial San

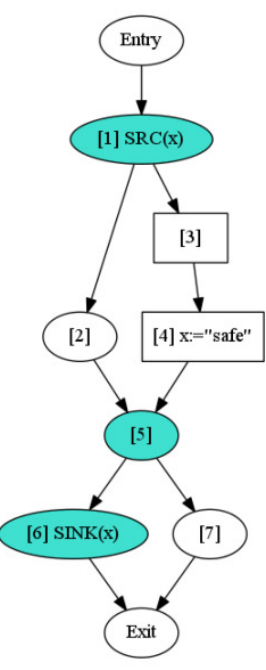

(d) Propagated San

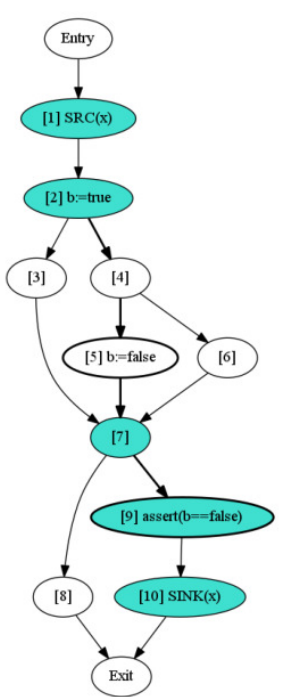

(e) Allowed

Figure 10: The process of calculating Path-oracle. 


\subsection{Path-oracle Guided Path Navigation}

Algorithm 4 shows an algorithm filtering path summaries with Path-oracle. At first, it filters infeasible paths proved by Allowed out. And then, if current nodes of summary are assert statements, it selects the branches to continue searching using Path-oracle.

Codes at 5-12 lines filter infeasible path out by reserving only paths in Allowed. An extended summary design including reserved path is described in Section 4.7. Code at line 8 in Algorithm 4 reserves paths in Allowed for each path summaries. In Figure 10(e), a path $\{4\}\{5\}\{7\}\{9\}$ is reserved. Naturally, unreserved paths, that is, infeasible paths will be excluded in the future summary calculation.

Codes at 14-30 lines seek effective next node using Pick and San. At first, if the branch condition's truth value is determined, it selects the branch having true assert condition. In the other case, it selects branches whose Pick is true or opposite branch has true San. In Figure 10 (b). branch $\{5\}$ is selected because $\{5\}$ is in Pick. In (d), branch $\{2\}$ is selected because $\{3\}$ is in San. In case neither Pick nor San exist, it selects one branch arbitrarily avoiding already pruned branch. Because both branches are not affective to vulnerable behaviour.

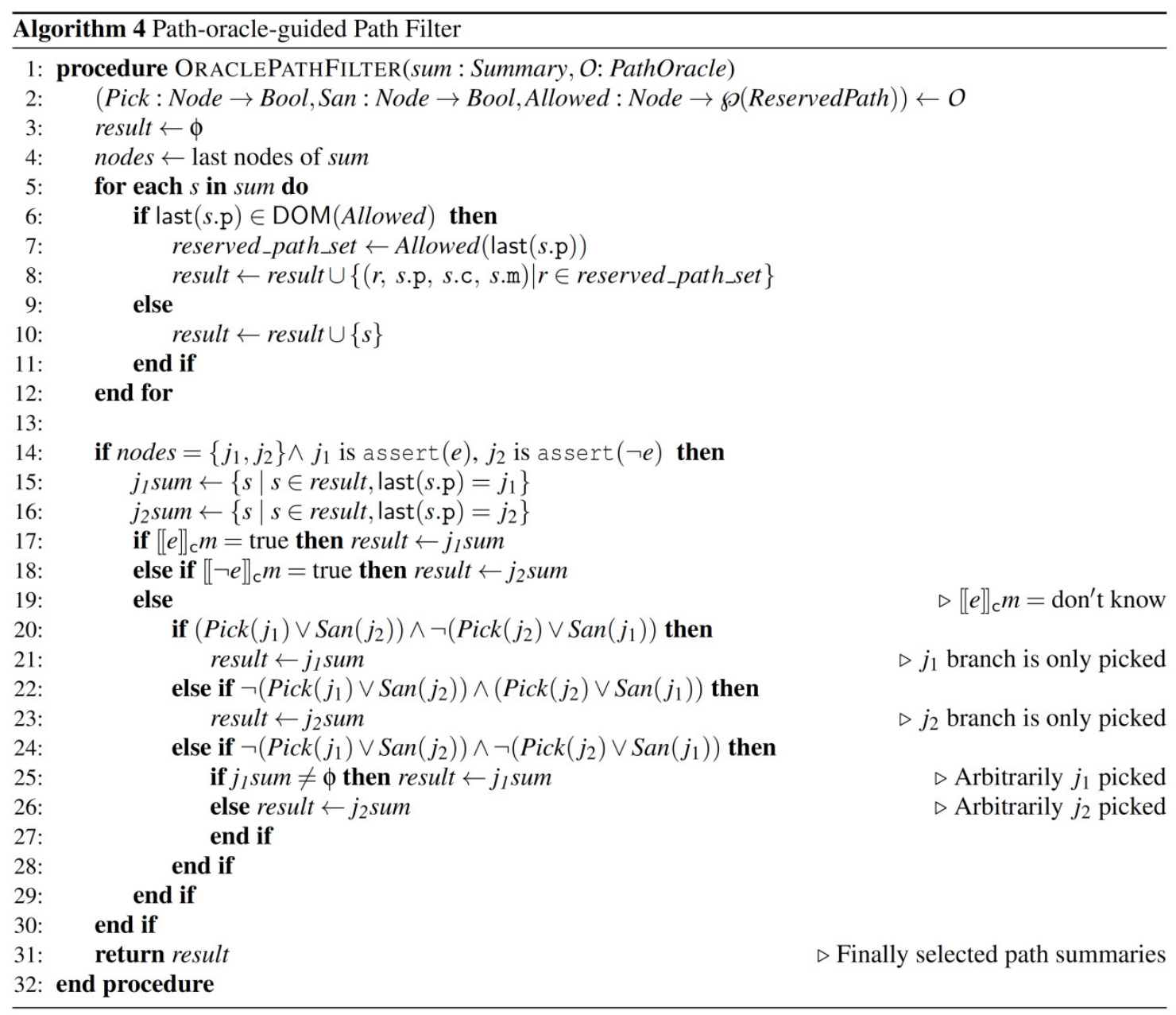


Figure 11 is an example of San is essential for path navigation. In code of line 7, GETREQUESTEDSESSIONID gives source information and then it goes to sink at line 11 . There are two branches between line 7,11. Both two branches are not nodes propagating the source information to the sink. However, true branch is actually safe branch. Path navigation should avoid sanitizer to detect the vulnerable path.

\subsection{Pre-analysis Technique Guided Path-sensitive Analysis for Security Vulnerability}

We present A Main-analysis using Path-oracle for its path navigation. Figure 12 shows a new Summary design. There are only minor changes. ReservedPath field is added in PathSummary. A path summary having reserved path should be calculated following the reserved path. It is shown in the Figure 13.

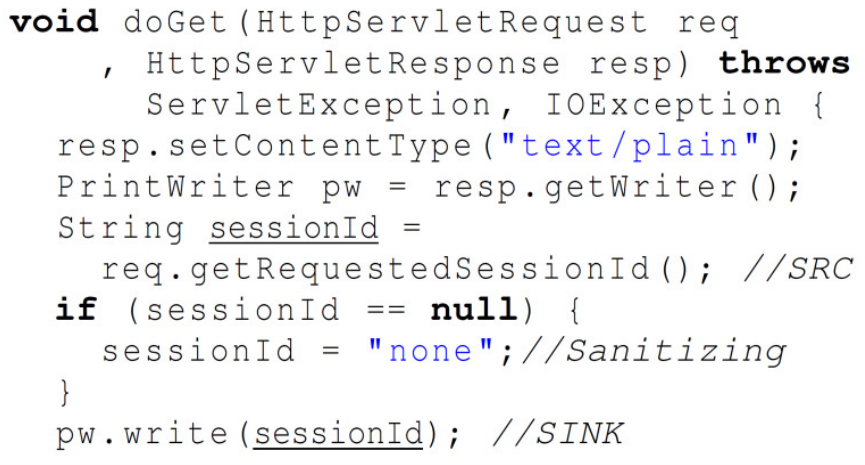

Figure 11: Example of code including a sanitizing branch from TestCoyoteAdapter.java of Tomcat

$$
\begin{aligned}
\text { Summary } & =\wp(\text { PathSummary }) \\
\text { PathSummary } & =\{\mathrm{r}: \text { ReservedPath }, \mathrm{p}: \text { Path } \\
\text { ReservedPath } & =(\text { Method } \times \text { Node }) \text { List } \\
\text { Path } & =(\text { Method } \times \text { Node }) \text { List } \\
\text { SConstraint } & =\text { SVal } \rightarrow \text { Constraint } \\
\text { SMemory } & =\text { SRef } \rightarrow \text { SVal }
\end{aligned}
$$

Figure 12: Summary of Main-analysis

$$
\begin{aligned}
& \llbracket s t m t_{i} \rrbracket_{\text {new }}(\phi, p, c, m) \\
& =\left\{\left(\phi, p^{\prime}, c^{\prime}, m^{\prime}\right) \mid\left(p^{\prime}, c^{\prime}, m^{\prime}\right) \in \llbracket s t m t_{i} \rrbracket(p, c, m)\right\} \\
& \llbracket s_{m} m t_{i} \rrbracket_{\text {new }}\left(p_{\mathrm{rsvd}}:: r, p, c, m\right) \\
& =\left\{\left(r, p^{\prime}, c^{\prime}, m^{\prime}\right) \mid \begin{array}{c}
\left(p^{\prime}, c^{\prime}, m^{\prime}\right) \in \llbracket s t m t_{i} \rrbracket(p, c, m) \\
p^{\prime}=p:: p_{\mathrm{rsvd}}
\end{array}\right\}
\end{aligned}
$$

Figure 13: Semantics of Main-analysis 
Algorithm 5 is extended algorithm of Algorithm 2 by the new semantics and ORACLE PATHFILTER algorithm. In line 8, the previous semantics is replaced by the new semantics. At line 9 ORACLEPATHFILTER algorithm is added. It reserves may-feasible paths for some path summaries and filters out non-vulnerable path summaries. WEIGHTPATHFILTER is preserved same as before to ensure the quality of the previous.

\section{EXPERIMENT RESULT}

We evaluate the proposed two analysis techniques by two experiments. First, we evaluate their ability to achieve high precision and recall on OWASP Benchmark. OWASP Benchmark is a benchmark to evaluate precision and recall of a vulnerability scanner. The result is presented in Section 5.1. Second, we evaluate the improvement by the pre-analysis technique on 12 real-world projects.

We implemented two analyzer for the experiments. One analyzer is implemented through the path-sensitive analysis technique. The other analyzer is implemented through the pre-analysis technique based on the first analyzer. We call the first one as Baseline analyzer and second one as Modified analyser. $t$ be published in the conference proceedings.

\subsection{Experiment 1: Precision and Recall}

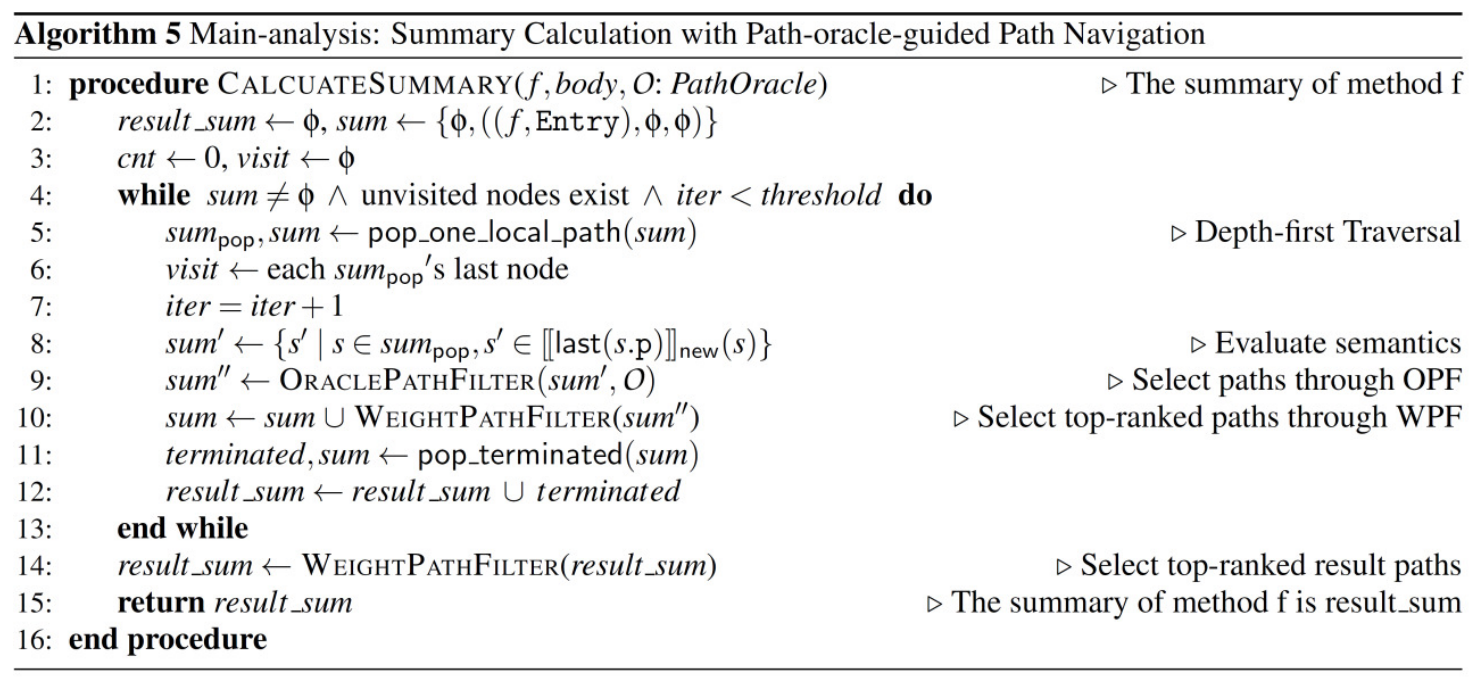

OWASP Benchmark ${ }^{1}$ is a benchmark to evaluate precision and recall of a vulnerability scanner. It consists of 2740 test-cases which may have one of 10 top vulnerabilities. The ratio of the number of safe test-cases to the number of unsafe test-cases is almost one to evaluate both precision and recall of a vulnerability scanner.

F-measure is a measure combining precision and recall. A F-measure is the harmonic mean of precision and recall. The F-measure is 100 when a scanner's both precision and recall are $100 \%$. The F-measure is 0 when a scanner's both precision and recall are $0 \%$.

\footnotetext{
${ }^{1}$ www.owasp.org/index.php/Benchmark, v1.2 beta
} 
Baseline analyzer gets F-measure 96 on OWASP Benchmark. An analyzer by our method may get high precision easily because of no restriction on symbolic state design. Table 1 shows that Baseline analyzer achieves precision 100\%. Although there are 98 false-negatives, it achieves high recall $93 \%$. The table shows that the introduced path navigation strategy is effective to scan vulnerabilities.

Modified analyzer gets the same F-measure as Baseline analyzer. The experiment result of Modified analyzer is exactly same as Table 1. Section 5.2 shows that the number of explored path reduced by 3.4 times. Nonetheless, the precision and recall are preserved.

Table 1: Evaluation of the path-sensitive analysis technique on OWASP Benchmark (TP=true positive, FN=false negative, $\mathrm{TN}=$ true negative, $\mathrm{FP}=$ false positive)

\begin{tabular}{|l|r|r|r|r|r|r|r|r|r|}
\hline \multirow{2}{*}{ Category } & \multicolumn{2}{|c|}{ Test query } & \multicolumn{9}{|c|}{ Result } & \multicolumn{3}{|c|}{ Score } \\
\cline { 2 - 9 } & unsafe & \multicolumn{1}{|c|}{ safe } & TP & FN & \multicolumn{1}{|c|}{ TN } & FP & recall(\%) & precision(\%) & F-measure \\
\hline Command Injection & 126 & 125 & 126 & 0 & 125 & 0 & 100 & 100 & 100 \\
Weak Cryptography & 130 & 116 & 97 & 33 & 116 & 0 & 75 & 100 & 85 \\
Weak Hashing & 129 & 107 & 89 & 40 & 107 & 0 & 69 & 100 & 82 \\
LDAP Injection & 27 & 32 & 27 & 0 & 32 & 0 & 100 & 100 & 100 \\
Path Traversal & 133 & 135 & 123 & 10 & 135 & 0 & 92 & 100 & 96 \\
Secure Cookie Flag & 36 & 31 & 36 & 0 & 31 & 0 & 100 & 100 & 100 \\
SQL Injection & 272 & 232 & 272 & 0 & 232 & 0 & 100 & 100 & 100 \\
Trust Boundary Violation & 83 & 43 & 83 & 0 & 43 & 0 & 100 & 100 & 100 \\
Weak Randomness & 218 & 275 & 203 & 15 & 275 & 0 & 93 & 100 & 96 \\
XPATH Injection & 15 & 20 & 15 & 0 & 20 & 0 & 100 & 100 & 100 \\
XSS & 246 & 209 & 246 & 0 & 209 & 0 & 100 & 100 & 100 \\
\hline Total & 1415 & 1325 & 1317 & 98 & 1325 & 0 & 93 & 100 & 96 \\
\hline
\end{tabular}

\subsection{Experiment 2: Improvement of Efficiency}

We construct a new benchmark containing up to one million lines of code to evaluate the preanalysis technique. They are listed in Table 2. Two of them: sparrowml and objchk are our internal java program. Other ten programs are open source projects composed of Java and jsp. Especially four of them: josso1, jdesurvey, broadleaf commerce, and alfresco are open source projects using Spring framework. The largest number of lines of code in the benchmark has about one million lines of code.

Baseline analyzer efficiently covers vulnerable paths. The size of codes in OWASP Benchmark is $161 \mathrm{~K}$ LOC. it analyzes the codes within 10 minutes. This is 1 Million LOC/hour phase.

Our experimental result presents the pre-analysis technique makes Baseline analyzer several times more efficient. In spite of additional pre-analysis, the total analysis time is reduced by several times. The analysis time of Modified analyzer dramatically decreases comparing to Baseline analyzer. It is reduced by about 6 times. The total analysis time is also reduced by 2.5 times. Many programs in the benchmark are analyzed about 4 times faster. Even javassist having the least effect is analyzed about 1.6 times faster.

Table 2 shows that additional true-positives are discovered by the new method. Although, the increased number of true-positives is not so large, but it is a meaningful number in comparison to the decreased number. Only tomcat loses one true-positive. The others lose nothing. There are no declines for josso1 when 38 new vulnerabilities are detected. Even tomcat who lose one true- 
positive gain 9 additional true-positives. Reduction of explored paths makes analysis time decrease, but rather precision increase.

Table 2: Evaluation of the pre-analysis technique on 2 internal private source projects and 10 open source projects.

\begin{tabular}{|c|c|c|c|c|c|c|c|c|c|c|c|}
\hline \multirow{3}{*}{ LOC } & \multirow{3}{*}{$\begin{array}{c}\text { Benchmark } \\
\text { (java+jsp) }\end{array}$} & \multicolumn{3}{|c|}{ Explored local path(\#) } & \multicolumn{5}{|c|}{ Analysis time(s) } & \multicolumn{2}{|c|}{ Alarm Diff } \\
\hline & & \multirow{2}{*}{ prev } & \multirow{2}{*}{ new } & \multirow{2}{*}{ ratio } & \multirow{2}{*}{$\begin{array}{l}\text { prev } \\
\text { main }\end{array}$} & \multicolumn{3}{|c|}{ new } & \multirow{2}{*}{ ratio } & \multirow{2}{*}{$\Delta \mathrm{TP}$} & \multirow{2}{*}{$\Delta \mathrm{FN}$} \\
\hline & & & & & & pre & main & total & & & \\
\hline $20 \mathrm{~K}$ & sparrowml & 6,134 & 638 & X9.6 & 138 & 12 & 7 & 19 & $\mathrm{x} 7.3$ & 0 & 0 \\
\hline $26 \mathrm{~K}$ & we & 962 & 337 & & 95 & 10 & 12 & 22 & 3 & 0 & 0 \\
\hline $32 \mathrm{~K}$ & objchk & 6,901 & 847 & x 8.1 & 181 & 15 & 25 & 40 & $\mathrm{x} 4.5$ & 0 & 0 \\
\hline $40 \mathrm{~K}$ & goatc & 5,470 & 1,137 & $\mathrm{x} 4.8$ & 67 & 17 & 13 & 30 & 2 & 0 & 0 \\
\hline $65 \mathrm{~K}$ & joss & 9,195 & 2,161 & $\mathrm{x} 4.3$ & 430 & 44 & 49 & 93 & $x 4.6$ & 38 & 0 \\
\hline $79 \mathrm{~K}$ & javass & 9,022 & 1,991 & $\mathrm{x} 4.5$ & 155 & 84 & 15 & 99 & $\mathrm{x} 1.6$ & 0 & 0 \\
\hline $83 \mathrm{~K}$ & roller & 12,277 & 4,904 & & 268 & 37 & 49 & 86 & 1 & 3 & 0 \\
\hline $88 \mathrm{~K}$ & jdesu & 3,075 & 2,358 & $\mathrm{x}$ & 277 & 23 & 40 & 63 & $x 4.4$ & 0 & 0 \\
\hline $156 \mathrm{~K}$ & struts & 22,085 & 5,847 & $\mathrm{x} 3.8$ & 689 & 147 & 110 & 257 & $\mathrm{x} 2.7$ & 0 & 0 \\
\hline $196 \mathrm{~K}$ & broadleaf c. & 25,911 & 7,140 & x3.6 & 1,618 & 161 & 228 & 389 & $\mathrm{x} 4.2$ & 4 & 0 \\
\hline $249 \mathrm{~K}$ & tomcat & 47,156 & 13,021 & x3.6 & 1,589 & 197 & 205 & 402 & $\mathrm{x} 4.0$ & 9 & 1 \\
\hline \multirow[t]{2}{*}{$969 \mathrm{~K}$} & alfresco & 107,839 & 35,399 & $\mathrm{x} 3.0$ & 7,384 & 2,291 & 1,454 & 3,745 & $\mathrm{x} 2.0$ & 1 & 0 \\
\hline & TOTAL & 256,027 & 75,780 & $\mathrm{x} 3.4$ & 12,891 & 3,038 & 2,207 & 5,245 & $\mathrm{x} 2.5$ & 55 & 1 \\
\hline
\end{tabular}

The number of detections of deep inter-path vulnerable paths increases. The paths of the additional true positives have more than 3 call-depth. The deepest inter-path has 5 call-depth with long inter-procedural path. Baseline analyzer detects long inter-paths which include many callsites frequently, but paths who have deep inter-paths rarely.

Another encouraging point is that the number of vulnerabilities detected among nested branches increases.

\subsection{Discussion}

Our path-sensitive analysis technique is scalable and has ability to achieve high precision and recall. Table 2 shows that an analyzer implemented by only the path-sensitive analysis technique is able to analyze 1 million LOC. Table 1 shows that the analyzer has high precision and recall.

The pre-analysis technique is a goal-oriented path navigation technique. Goals are clearly reflected by Path-oracle in the algorithm. Path-oracle guided path navigation saves unnecessary path searches. The detection rate of vulnerabilities having deep inter-paths or many branching increases.

Reduction of the number of explored paths leads the time reduction. Although the reduced number of explored paths and the reduced time are not exactly proportional, but most of them are highly correlated. As an unusual case, jdesurvey's number of explored path is reduced by only 1.3 times when the time reduced by 4.4 times. The Modified analyzer avoided paths that are meaningless but time-consuming.

Additional false-negatives may be raised because of two reasons. First, the proposed algorithm calculates approximate fixed point of a Pre-analysis semantics. A Pre-analysis may miss vulnerable nodes. Second, the heuristic for effective interval is not optimal. A Pre-analysis may fail to find infeasible paths. 
A disadvantage of the pre-analysis technique occurs when modifying the design of target analysis. Simultaneous modifications of a Pre-analysis semantics and the Main-analysis semantics are not always necessary, but a developer should always examine whether modification of the Pre-analysis semantics is necessary or not. Changes in core design of a Main-analysis require a design change in the Pre-analysis. An incorrect Pre-analysis makes the Main-analysis lose opportunity to detect vulnerable paths.

\section{FUTURE WORK}

A more researches for heuristics to find effective intervals are required. The effective interval classifier works using heuristics rather than logical methods. We do not know our heuristics are close to the optimal although the heuristics are effective. How to make effective interval classifier is a key issue left.

Effective interval classifier trained by machine learning algorithm may be used. [10] presents a method for learning a classifier which select good analysis parameters of a target code via Bayesian optimization. The advantage of this method is that Bayesian optimization do not require training data but just an objective function which is path-sensitive constant propagation in this case. Supervised learning algorithm like SVM requires training data and obtaining the training data, effective intervals in this case, is not easy.

We have a plan to improve effective interval classifier using Bayesian optimization. Feature vector is a crucial factor for effective learning. Most of the research will be finding the good feature vector. We will look at many codes and study important features for effective interval.

\section{CONCLUSIONS}

We have given a scalable path-sensitive analysis technique for many types of security vulnerabilities with high precision and recall. The technique do not restrict design condition of symbolic state for making highly precise vulnerability scanner to be easy. Scalability is achieved by a path navigation heuristic only. Experimental results show that it can analyze 1 million lines of code. Despite the path navigation heuristic filters out many paths for scalability, it does not lose recall.

We have presented a pre-analysis technique improves the efficiency of the path-sensitive analysis. Both analysis time and recall are improved by the method. Experimental results show that our technique reduces the number of explored paths by 3.4 times. Due to the reduction, the analysis time reduced by 2.5 times in spite of the additional cost for a pre-analysis. Despite the number of explored paths is reduced, false negatives are reduced.

\section{ACKNOWLEDGEMENTS}

This work was supported by Institute for Information \& communications Technology Promotion(IITP) grant funded by the Korea government(MSIP). (No.R0190-15-1099, Development of an integrated management system and a security testing system that enables interaction between security vulnerability detection technologies in development and operation phases of web application) 


\section{REFERENCES}

[1] Balakrishnan, G., Sankaranarayanan, S., Ivani, F., Wei, O., and Gupta, A. (2008). Slr: Path-sensitive analysis through infeasible-path detection and syntactic language refinement. In Static Analysis. SAS 2008. Lecture Notes in Computer Science, vol 5079. Springer.

[2] Gizem, Aksahya \& Ayese, Ozcan (2009) Coomunications \& Networks, Network Books, ABC Publishers.

[3] Cousot, P. and Cousot, R. (1977). Abstract interpretation: A unified lattice model for static analysis of programs by construction or approximation of fixpoints. In Proceeding POPL ' 77 Proceedings of the 4th ACM SIGACT-SIGPLAN symposium on Principles of programming languages. ACM.

[4] Das, M., Lerner, S., and Seigle, M. (2002). Esp: Pathsensitive program verification in polynomial time. In Proceeding PLDI '02 Proceedings of the ACM SIGPLAN 2002 conference on Programming language design and implementation. ACM.

[5] Dillig, I., Dillig, T., and Aiken, A. (2008). Sound, complete and scalable path-sensitive analysis. In PLDI '08 Proceedings of the 29th ACM SIGPLAN Conference on Programming Language Design and Implementation. ACM.

[6] Gutzmann, T., Lundberg, J., and Lowe,W. (2007). Towards path-sensitive points-to analysis. In Seventh IEEE International Working Conference on Source Code Analysis and Manipulation (SCAM 2007). IEEE.

[7] Jaffar, J., Murali, V., Navas, J. A., and Santosa, A. E. (2012). Path-sensitive backward slicing. In Proceeding SAS'12 Proceedings of the 19th international conference on Static Analysis. ACM.

[8] Le, W. and Soffa, M. L. (2008). Marple: A demand-driven path-sensitive buffer overflow detector. In Proceeding SIGSOFT '08/FSE-16 Proceedings of the 16th ACM SIGSOFT International Symposium on Foundations of software engineering. ACM.

[9] Li, H., Kim, T., Bat-Erdene, M., and Lee, H. (2013). Software vulnerability detection using backward trace analysis and symbolic execution. In 2013 International Conference on Availability, Reliability and Security. IEEE.

[10] Navabi, A., Kidd, N., and Jagannathan, S. (2010). Pathsensitive analysis using edge strings. In DEPARTMENT OF COMPUTER SCIENCE TECHNICAL REPORTS. Purdue University.

[11] Oh, H., Yang, H., and Yi, K. (2015). Learning a strategy for adapting a program analysis via bayesian optimisation. In OOPSLA 2015 Proceedings of the 2015 ACM SIGPLAN International Conference on Object-Oriented Programming, Systems, Languages, and Applications. ACM.

[12] Santelices, R. and Harrold, M. J. (2009). Spa: Symbolic program approximation for scalable pathsensitive analysis. In CERCS Technical Reports [193]. Georgia Institute of Technology.

[13] Vojdani, V. and Vene, V. (2009). Goblint: Path-sensitive data race analysis. In Annales Univ. Sci. Budapest., Sect. Comp.

[14] Winter, K., Zhang, C., Hayes, I. J., Keynes, N., Cifuentes, C., and Li, L. (2013). Path-sensitive data flow analysis simplified. In Formal Methods and Software Engineering. ICFEM 2013. Lecture Notes in Computer Science, vol 8144. Springer. 
[15] Xie, Y. and Aiken, A. (2005). Context- and path-sensitive memory leak detection. In ESEC/FSE-13 Proceedings of the 10th European software engineering conference held jointly with 13th ACM SIGSOFT international symposium on Foundations of software engineering. ACM.

[16] Zheng, Y. and Zhang, X. (2013). Path sensitive static analysis of web applications for remote code execution vulnerability detection. In Proceeding ICSE '13 Proceedings of the 2013 International Conference on Software Engineering. IEEE.

\section{AUTHORS}

Dongok Kang

Working at Fasoo.com R\&D Center as an engineer

M.S. and B.S in Computer Science and Engineering from Seoul National University

Minsik Jin

Working at Fasoo.com R\&D Center as a team leader.

M.S. and B.S. in Computer Science and Engineering from Seoul National University
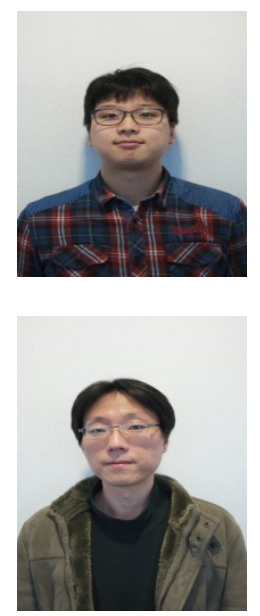


\title{
CLASSIFICATION ALGORITHMS FOR THE DETECTION OF THE PRIMARY TUMOR BASED ON MICROSCOPIC IMAGES OF BONE METASTASES
}

\author{
Slađan Kantar ${ }^{1}$, Aleksandar Pluškoski ${ }^{2}$, Igor Ciganovic ${ }^{3}$ and Jelena \\ Vasiljević $^{4}$ \\ ${ }^{1,2,3}$ Master student at "The Faculty of Computer Science" \\ ${ }^{4}$ Professor at "The Faculty of Computer Science", \\ University of Belgrade, Serbia \\ Science Assistant in "Institute Mihajlo Pupin"
}

\begin{abstract}
This paper presents the analysis of techniques for microscopic images in order to find a primary tumor based on the of bone metastases. Was done algorithmic classification into three groups, kidney, lung and breast. In order to speed up the treatment of the patient and easier for doctors and therefore reduce room for human error. Digital microscope images of bone metastases were analyzed, for which it is known that the primary tumor is in one of the three human organs: kidney, lung or breast. We tested several solutions for classification, were tested two methods of image analysis. Multifractal analysis and convolutional neural network. Both methods were tested with and without preprocessing image. Results of multifractal analysis were then classified using different algorithms. Images were processed using CLAHE and $k$ means algorithm. At the end, the results obtained using a variety of techniques are presented.
\end{abstract}

\section{KEYWORDS}

Cancer classification, Microscopic images, Image preprocessing, Multifractal analysis, Classification algorithms

\section{INTRODUCTION}

Cancer is the leading cause of death in the world. Every year more and more people being diagnosed, and advances in medicine, unfortunately, not fast enough, although in recent years much has been done. In computer science, many scientific papers dealing with automation of diagnosis and determining ways of treating cancer. Our work deals with the analysis of various techniques for algorithmic classification (determination) of a primary tumor based on the microscopic images of metastases in bones.

In the last few years, machine learning algorithms experienced the large popularization and expansion. It is increasingly used in various fields of industry and science. They became part of

Natarajan Meghanathan et al. (Eds) : ACSIT, SIPM, FCST, CoNeCo, CMIT - 2017

pp. 41- 56, 2017. (C) CS \& IT-CSCP 2017

DOI : $10.5121 /$ csit.2017.70804 
the products we use every day. Also, used in medicine all over. However, these algorithms are statistically and require very large amounts of data for their application. In recent years, more and more of data become available and is the main cause of the increasing use of this type of algorithms. The basic idea of each of these algorithms is based on large amount of data to notice statistical similarity within class from training data and then attempt to extrapolate "learned" to new data and classified them into one of the given class.

When it comes to image analysis with machine learning algorithms, convolutional neural networks are unavoidable, but in medicine is also applied fractal and multifractal analysis, due to the observed fractal characteristics of medical images. Convolution neural network classification algorithm presents whole classifying algorithm that take images from the input and classifying it into one of the classes. In both cases, the images that are analyzed may be a "raw", obtained directly from the microscope, or preprocessed. We tested both cases, in order to compare results.

\section{MODELS AND METHODS}

Detection and classification of cancer using microscopic images with biopsy is the arduous and complex task. Many different techniques is entered in the proposed method, which includes, changing the contrast of the image, segmentation of the cell, multifractal analysis, and at the end classification. In the Figure1 show two methods for classification are presented. First proposed, for improving the microscopic images of tissue use CLAHE algorithm, which is then passed through the K-means algorithm for segmentation of the cell. With this method we have highlighted important biological and clinical forms, as well as morphological features, which include shades of gray, color segmentation and segmentation in texture ${ }^{[1][2]}$. In the end, it applies multifractal analysis and some clustering algorithm. The second proposal is that on microscopy images of biopsy put direct through multifractal analysis, and then clustering algorithm. These algorithms were tested on randomly selected 1050 (350 in each group - kidney, lung, breast) microscopic image of biopsy. At the end it was measured and analyzed performance of each classification algorithm. Below is described the work of each of the algorithms used in this experiment.

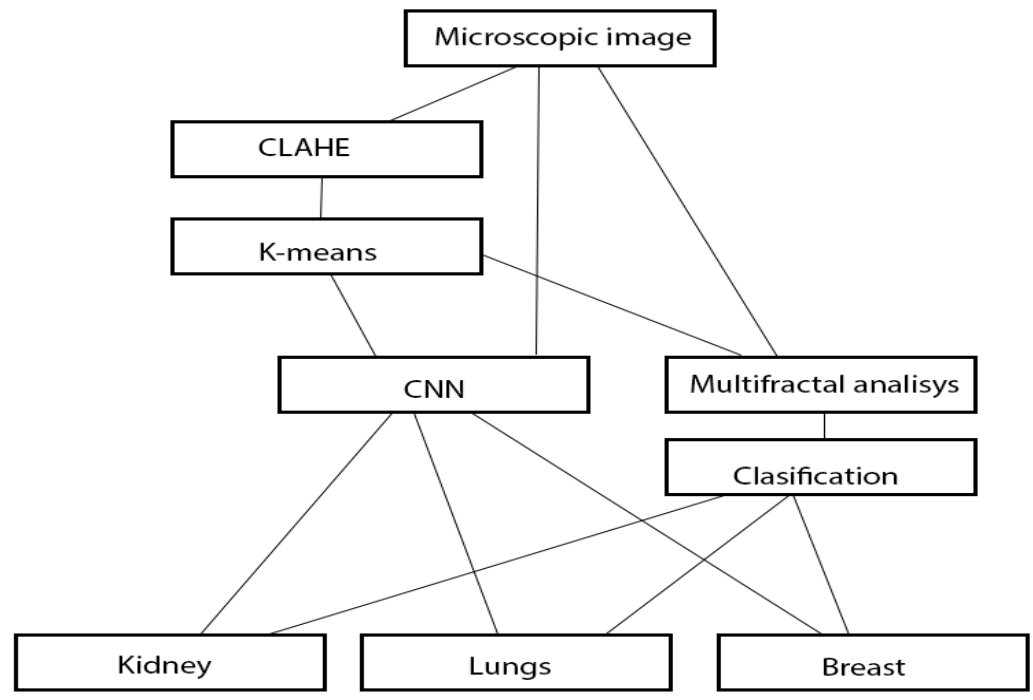

Figure 1 


\subsection{Image Enhancement}

The main purpose was to reduce noise and to make higher contrast, so to express for use important regions. During the shooting of microscopic images it occurs image deformation due to small focal length (fisheye) and pour brightness. Thus, images are over or underexposed and even unclear edges. Due to all of this we have used CLAHE algorithm to enhance microscopic images. Figure 2 show's us image before and after CLAHE algorithm.

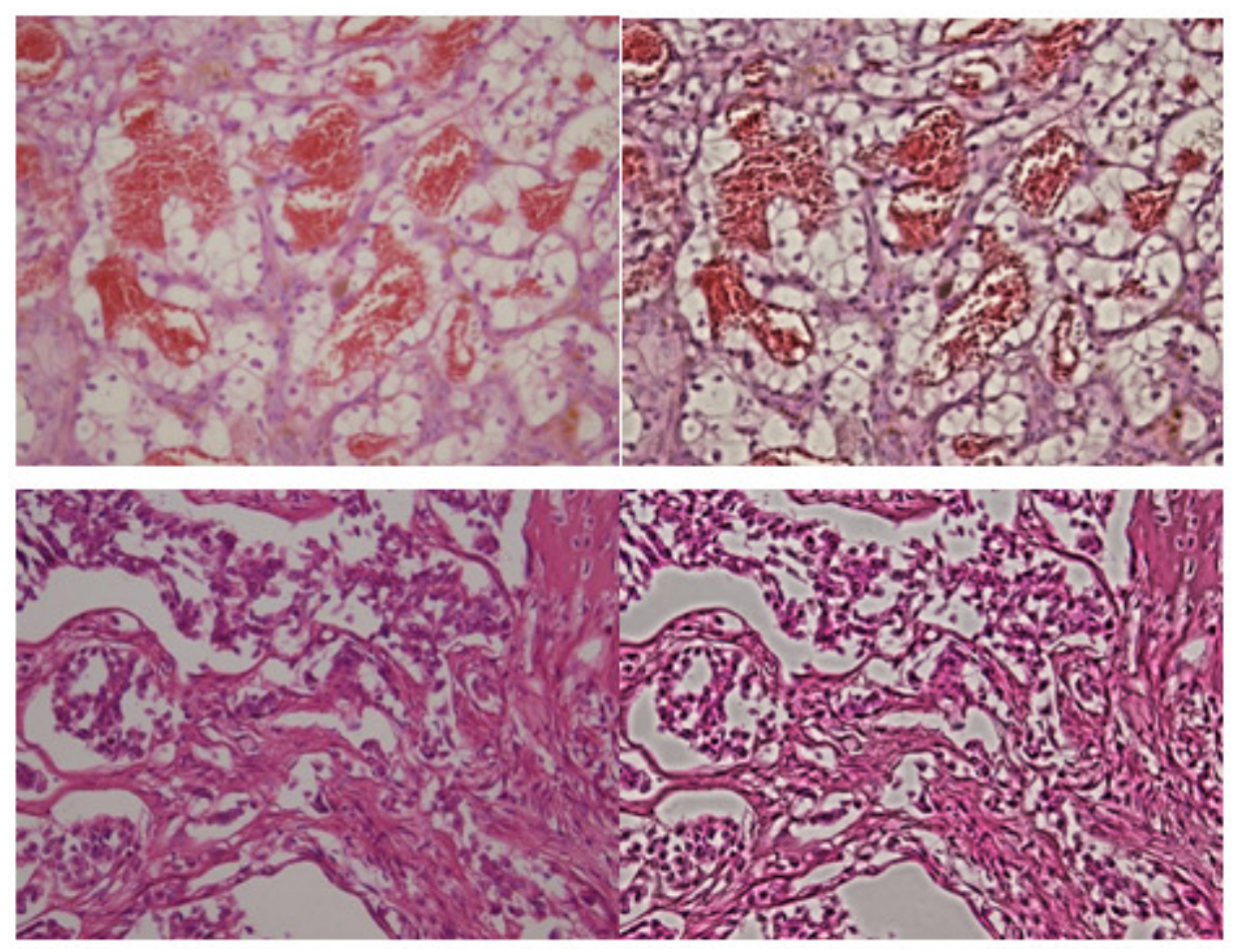

Figure 2. Left images are original, images on the right are after CLAHE algorithm.

\subsection{Segmentation}

There are a two ways of images segmentation, based on cells, cytoplasm and nucleus. First way is to use human but it will take too much time and there is the possibility for human error. Second way, is to automate this process by using some types of algorithms. We have tested a lot of algorithms, but the best, and only useful, was k-means colors based segmentation. K-means is vector segmentation, first developed in signal processing. K-means algorithm first partition $n$ objects into $\mathrm{k}$ clusters ${ }^{[3]}$. Our microscopic images are 8-bit color. That means the maximum number of colors that can be displayed at any one time is 256 . So we have clustered into 3 clusters $(\mathrm{k}=3)$. Now each picture is represented by three colors. With this method, we lose some data, but at the same time we simplifying model that is used for image classification. 

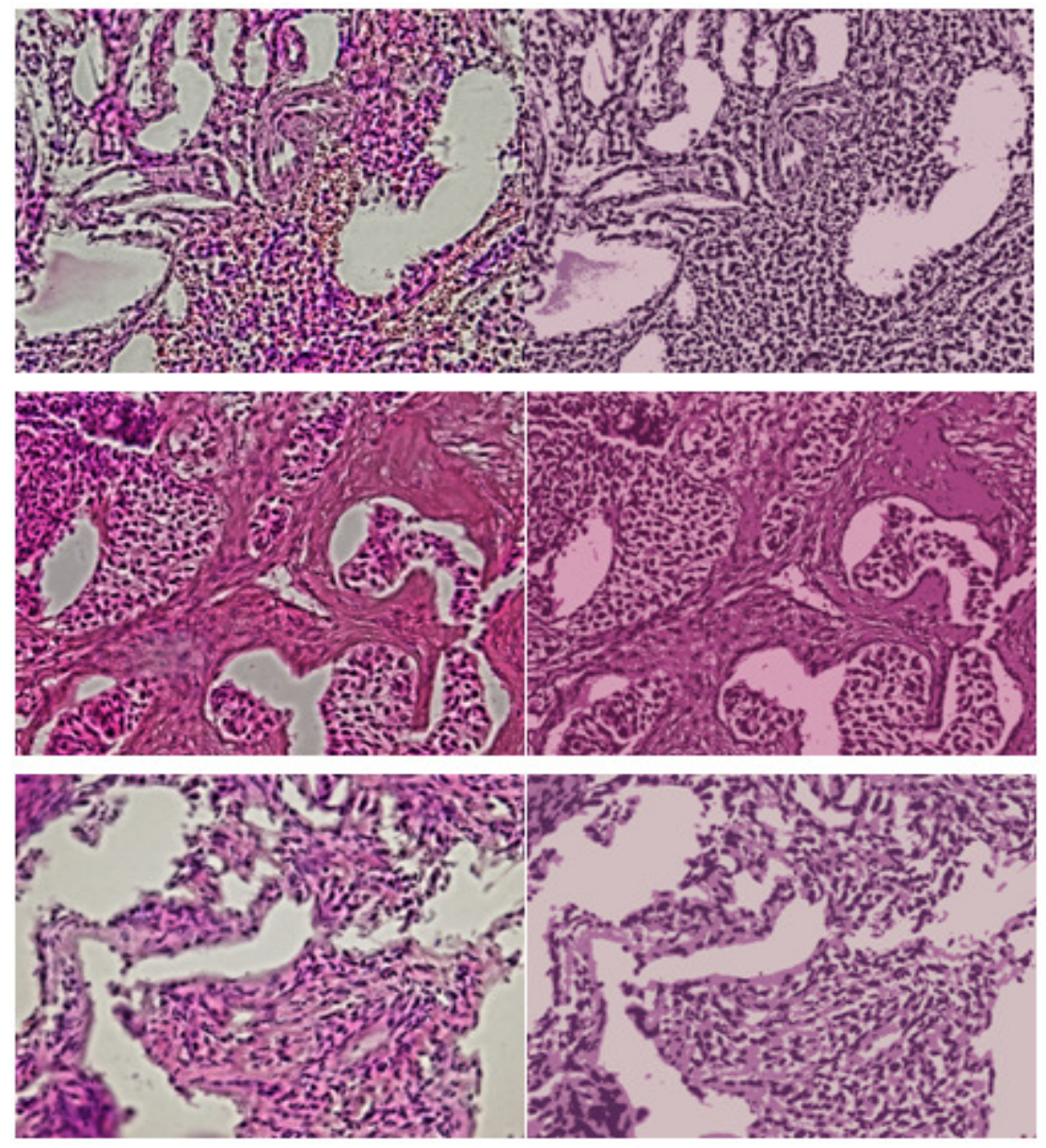

Figure 3. Images on the left before and on the right after k-means algorithm. Going from the top down, there are kidney, breast and lung.

\subsection{Multifractal Analysis}

For each of these three groups, 350 were selected Image (Total 1050). Multifractal analysis of digital medical images was done using ImageJ free program, and plug-in FracLac ${ }^{[4]}$. The parameters that were passed to the program are as follows: $Q$ in the range from -5 to +5 with step Qs of 0.25 , number of scans per image was is April 1st.

\subsection{Classification Algorithms}

Classification is the most common task in machine learning. It's a problem of identifying the items belonging to a predefined set. The classification of an object is based on finding similarities with predefined objects that belongs to different classes. When classifying each object is assigned to a class, with a certain probability of accuracy. Classification algorithm has the task of creating model based on training data which to exert new classification of unknown object. In classification the number of classes is know in advance and is not changeable.

It this paper it was used python open source library scikit-learn 0.18 . It was written in python, C and $\mathrm{C}++$. Featuring a variety of algorithms for classification such as SVM, Nearest Neighbors, 
Naive Bayes, Decision Trees, Ensemble methods; for regression and clusterization such as: KMeans and Spectral clustering ${ }^{[5][6]}$.

\subsubsection{Linear Discriminant Analysis}

LDA is a method often used is statistics and machine learning to find a vector (span or linear combination) that separates two or more classes. Result may be used as linear classifier or dimension reduction before applying other well-known classifiers.

LDA multi class classifier is generalization of LDA two class classifier that can work with arbitrarily number of classes. Multi class LDA is based on two scatter matrices: within class scatter matrix and between classes scatter matrix. Let's assume $\mathrm{x}_{1}, \ldots, \mathrm{x}_{\mathrm{n}}$, -is training data and their class labels are $\mathrm{y}_{1}, \ldots, \mathrm{y}_{\mathrm{n}}$, then scatter matrix within class is:

$$
\mathbf{S}_{w}=\sum_{i=1}^{n}\left(\mathbf{x}_{i}-\boldsymbol{\mu}_{y_{i}}\right)\left(\mathbf{x}_{i}-\boldsymbol{\mu}_{y_{i}}\right)^{T}
$$

Where $\mu_{\mathrm{k}}$ is mean values for class $\mathrm{k}$.

Scatter matrix between classes can be written as:

$$
\mathbf{S}_{b}=\sum_{k=1}^{m} n_{k}\left(\boldsymbol{\mu}_{k}-\boldsymbol{\mu}\right)\left(\boldsymbol{\mu}_{k}-\boldsymbol{\mu}\right)^{T}
$$

Here $m$ is number of classes, $m$ is mean value, and $n_{k}$ is number of samples in class $K$.

Then, multi class LDA can be written as a problem that find vector w which maximize difference between classes scatter and within class scatter.

$$
\hat{\mathbf{w}}=\underset{\mathbf{w}}{\operatorname{argmax}} \frac{\mathbf{w}^{T} \mathbf{S}_{b} \mathbf{w}}{\mathbf{w}^{T} \mathbf{S}_{w} \mathbf{w}}
$$

Generally, we need maximum $\mathrm{m}-1$ vector to get discrete values for $\mathrm{m}$ classes. We can write it like:

$$
\mathbf{S}_{b} \mathbf{w}=\lambda \mathbf{S}_{w} \mathbf{w}
$$

This algorithm isn't recommended when we have a lot of classes, due to high complexity of calculating ${ }^{[7]}$.

\subsubsection{Gaussian naive Bayes}

Naïve Bayes classifiers are a set of algorithms that uses Bayes theorem, assuming that training data is independently of each other. It's often used as basic classifier, because it's easy and fast to implement. This algorithm is degraded due to poor performance yet with good data preprocessing and well-tuned parameters it can be as good as SVM. ${ }^{[8][9]}$ 
There are a few types of naïve Bayes classifiers and difference between them is how they make distribution $\mathrm{P}(\mathrm{xi} \mid \mathrm{y})$. However in nature random things aren't so random. If you look closely you will see some regularity and in the most of time it will be Gaussian probability, or often called normal distribution or bell curve due to its shape. Thus, Gaussian naïve Bayes classifier is one of the most widely used. ${ }^{[5][6][10]}$ Let's assume that $\mathrm{X}$ is training data and $\mathrm{Y}$ is array of all classes which Xi can belongs to. First of all, we split training data by classes and for each class we find variance $2 \mathrm{y}$ and mean value $\mathrm{y}$. Then probability distribution of class $\mathrm{Xi}$ is:

$$
P\left(x_{i} \mid y\right)=\frac{1}{\sqrt{2 \pi \sigma_{y}^{2}}} \exp \left(-\frac{\left(x_{i}-\mu_{y}\right)^{2}}{2 \sigma_{y}^{2}}\right)
$$

\subsubsection{SVM (SVC)}

Support Vector Machine (SVM) is popular method for supervised machine learning for classification, regression and other machine tasks. SVM usually gives very accurate predictions, and they do not need too much parameters adjustment. They also are not sensitive on data size.

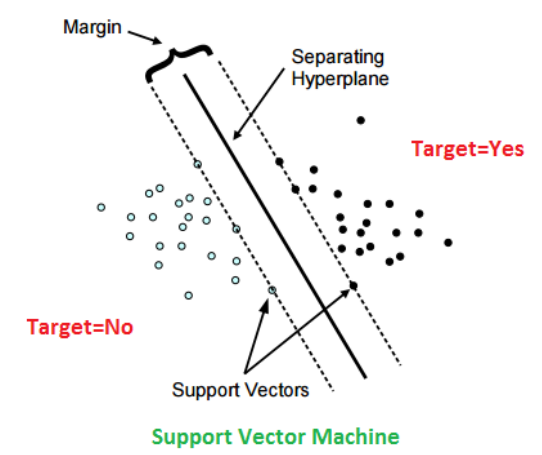

Figure 4. Schema of SVM algorithm

SVM first finds decision boundary. For this task it does not need all the training data. It's take data that are very close to other but are in class. Dots that are exactly on the boundary we call them support vectors, and that lines we call support planes. As we have sad earlier, we need boundary data, and now we calling them support vectors. By use of them we calculate separating (optimal) hyperplane.

How algorithm calculate boundary? First two hyperplanes that best represent data. For example, white dots (Target=No) are represent like $w^{T} x+b_{0}>1$, while black dots (Target=Yes) are represent like $w^{T} x+b_{0}<-1$, where is $\mathbf{w}$ weight vector, $\mathbf{x}$ is set of training data i.e. vectors, and $\mathbf{b}_{\mathbf{0}}$ is bias. Weight vector decides orientation of decision boundary while bias decides its location. Now decision boundary is in the midway of these two value (hyperplanes). ${ }^{[1]}$

SVC algorithm is a wider set of code which is capable now just calculating locations of hyperplanes it's also classifying data inside. SVC as a input take array that classifying into $\mathrm{n}$ classes. ${ }^{[5][6]}$ 


\subsubsection{K neighbors}

$\mathrm{K}-\mathrm{NN}$ is non parametric method for classification and regression, which is successful in cases where decision edge is irregular. ${ }^{[12]}$ Input in this algorithm is training data, which we get using multi fractal analysis. Output is the class to which it belongs. An object belong to that class, which have the most of his $\mathrm{k}$ neighbors $(\mathrm{k}>0)$. Parameter $\mathrm{k}$ is defined before starting an algorithm. If $\mathrm{k}=1$, then output is a class, which that one nearest object belongs. Distance between input data (points) can is calculated in different ways, but usually it is Euclid distance. To find right parameter $\mathrm{k}$ is complex process. It depends on type of training data. If it's $\mathrm{k}$ large, then we suppers noise, but on the same time we loses that precise border between classes. ${ }^{[5][6]}$ In this paper, we have three classes, based on a type of tissue (kidney, breast, and lung).

\subsubsection{Decision Tree}

DT is non parametric supervised learning used for classification and regression. The goal is to create a model that predicts the value by learning simple decision rules concluded from the training data. ${ }^{[5][6]}$ DT easy manipulate with big data and this makes it good for hyperspectral data. ${ }^{[13]}$ It is consists of nodes and edges. Each node is connected with a set of possible answer and in this way from input set of data we make smaller subset. Each represent possible test cases. DT like other machine learning algorithms uses recursion to make decision rules for classification from the training data. ${ }^{[14]}$

\subsubsection{Random Forests}

RF ensemble learning method for classification and regression. In RF each decision tree is built during the training, and output is class that is most times appear as output on every single tree. ${ }^{[6][15]}$ This means that makes the diverse set of classifiers, by introducing randomness, at the very design RF classifier. RF pick random vector $(X, Y)$ from training set. Vector $X=\{X 1, \ldots, X n\}$ and $\square \mathrm{X} \in \mathrm{R}+$ contains predictors and $\mathrm{Y} \in \overline{\mathrm{Y}}$ where $\overline{\mathrm{Y}}$ is class label or a numerical response. Classifier $\mathrm{t}$ is a mapping:

$$
\mathrm{t}: \mathrm{R}^{+} \in \overline{\mathrm{Y}}
$$

In RF, every tree in forest depends on the one random vector from training data and is equal probability of distribution for all trees.

\subsubsection{Convolutional Neural Network}

A convolutional neural network $(\mathrm{CNN})$ is a type of neural network that is used primarily for pattern recognition. It is mostly used in image recognition problems. We will talk about two dimensional convolutional neural network, but in practice number of dimensions that we can use are not limited. The idea behind $\mathrm{CNN}$ is to make neural network invariant to certain input transformations. This is the problem with fully connected neural networks, because layers are fully connected with each other which removes spatial information of the input.

CNN involves special convolutional and pooling (sub-sampling) layers. Output of which is usually flattened (transformed form matrix to vector) and used as input for fully connected network for classification. The architecture of $\mathrm{CNN}$ is shown in Figure 5. Convolution and sub- 
sampling operations can be repeated as many times as needed. There are three basic principles behind $\mathrm{CNN}$, local receptive fields, weight sharing and sub-sampling.

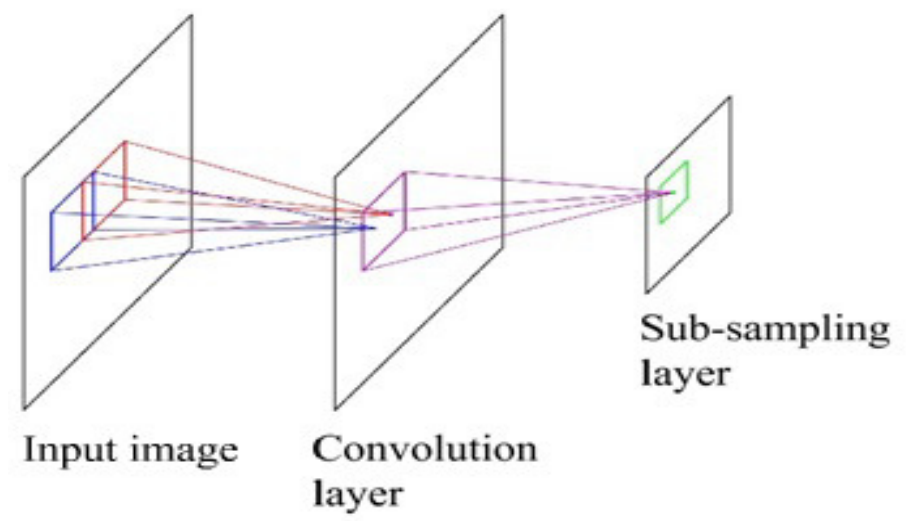

Figure 5. The architecture of a standard CNN. It shows a convolutional layer and is followed by a pooling layer.

\subsubsection{Local receptive fields}

In the fully connected feed forward neural network every neuron from one layer is connected to every neuron from the next. In CNN this is not the case. Every input in a convolutional layer is only connected to a small field of the previous layer. This is called local receptive field. In this way we preserve spatial information from the input. Figure 6. illustrates this idea. Here we have the first neuron of the convolutional layer connected only to nine pixels in the small $3 \times 3$ area of the input layer. ${ }^{[11]}$

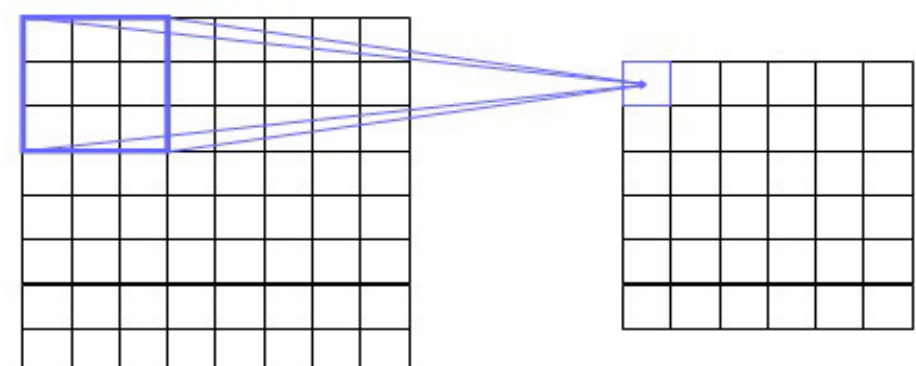

Input layer

Convolutional layer

Figure 6. An example of local receptive fields on a convolutional layer.

\subsubsection{Weight sharing}

Each convolutional layer is organized into multiple parallel hidden layers called feature maps. Neurons from different feature maps with the same index are always connected to the same area of the input. The idea of weight sharing is that all the neurons from the same feature map share the same weights, also called filter or kernel. Figure 7.Illustrates this. ${ }^{[16]}$ 


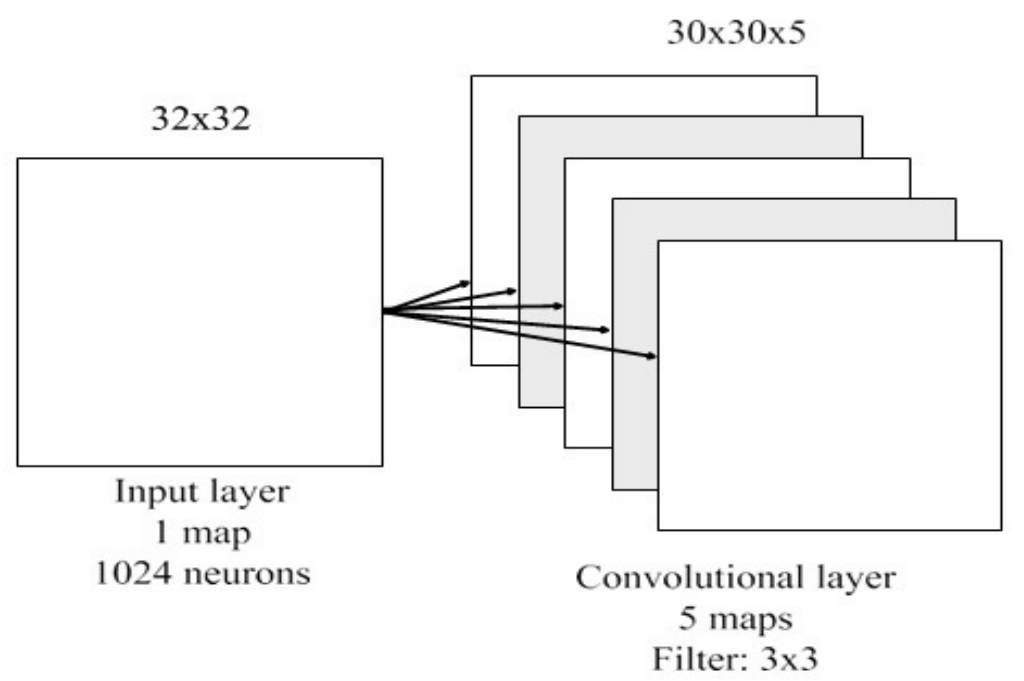

Figure 7. An example of a convolutional layer. The filter size is $3 \times 3$ and 5 feature maps.

\subsubsection{Sub-sampling}

Sub-sampling layer, also called pooling layer, is usually used immediately after a convolutional layer. Purpose of pooling layer is to create translation invariant features. This is done by computing statistical information of the activations from receptive field in the feature map of the convolutional layer. Figure 8.Illustrates process of sub-sampling. ${ }^{[16]}$

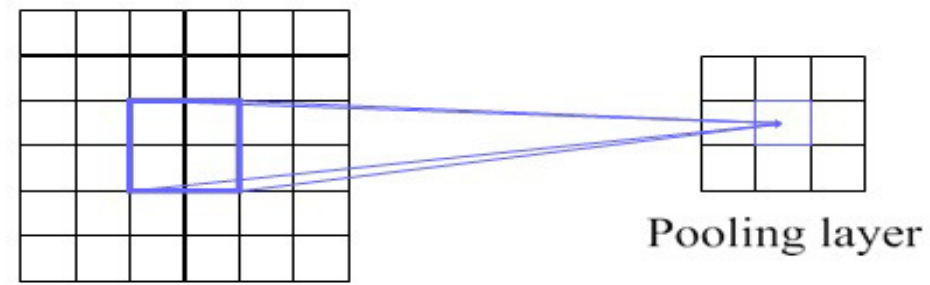

\section{Convolutional layer}

Figure 8. An example of a pooling layer in a feature map. This example uses $2 \times 2$ pooling size.

\section{RESULTS AND ANALYSIS}

\subsection{CNN}

We used convolutional neural network with relatively simple architecture built using TensorFlow framework. Consisting of up to six convolutional layers, all paired with pooling layer. 'ReLU' was used as the activation function. Parameters used for the convolutional layers were strides $=$ $[1,1,1,1]$ and padding = 'SAME' and for the pooling layer strides $=[1,1,1,1]$, $\mathrm{ksize}=[1,2,2$, 1] and padding = 'SAME'. Number of feature maps was calculated using formula:

$$
n=2^{4+i}
$$


where ' $i$ ' is index of the current convolutional layer. The output of the last pooling layer was used as an input into fully connected network consisting of two hidden layers and an output layer. First fully connected layer had $30 \%$ dropout rate, to reduce overfitting. Activation function used for hidden layers was 'sigmoid' and 'softmax' was used for output. 'AdamOptimizer' was used to train the network. Network was trained using different hyperparameters and using two data sets, processed and raw images. Images in both data sets were resized to $300 \times 226$ resolution, to reduce network complexity. Results are show in the Figure 9 and 10, showing accuracy progression over time for training using raw and preprocessed data sets respectively. Legends are shown separately for better visibility. Different colored lines represent different learning rates used and different number of convolutional layers.

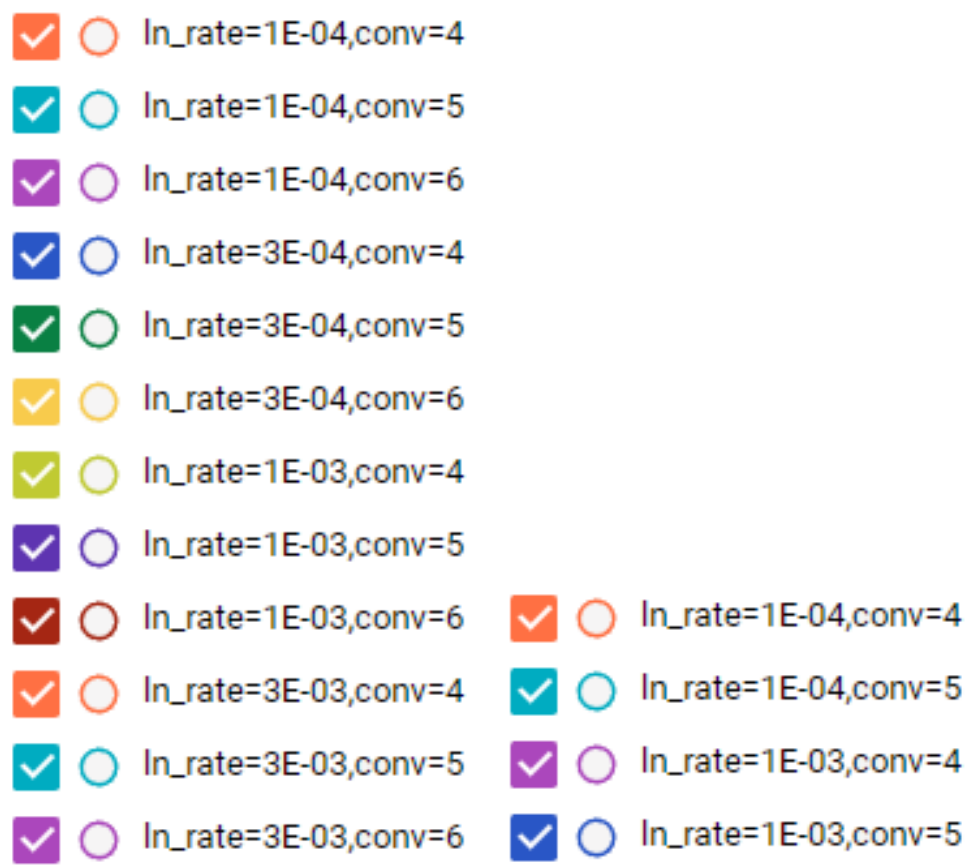

Figure 8. Legend the left for the plot representing accuracy for raw data set.Legend on the right for the plot representing accuracy for preprocessed data set.

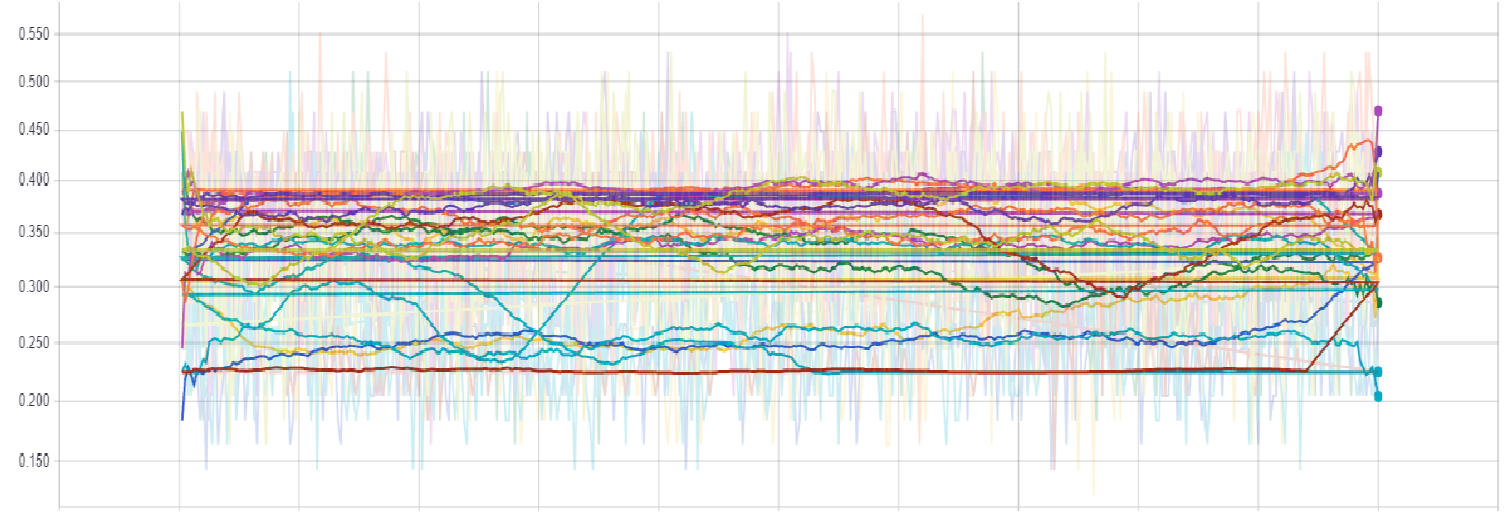

Figure 9. Accuracy over time for raw data set. 


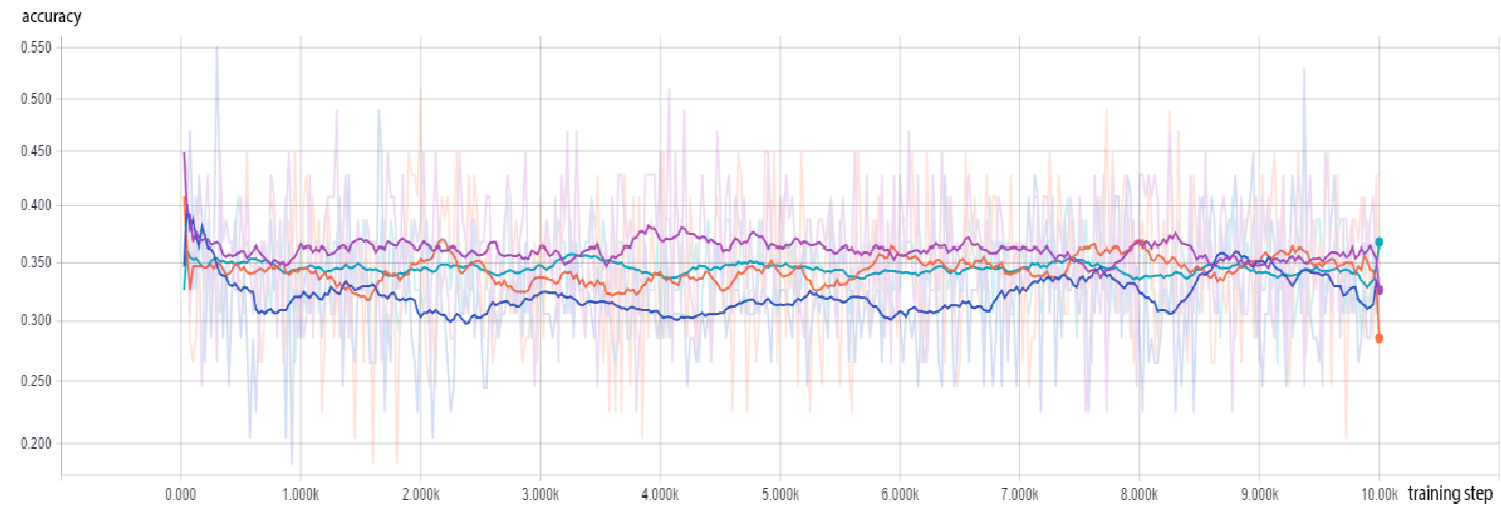

Figure 10. Accuracy over time for preprocessed data set.

As we can see from the graphs, simple convolutional neural networks are not a good solution to this kind of problems. We can see that some configurations of hyperparameters perform better than other. But with all configurations network converges very quickly and does not continue to learn new features. From the results we can see that there is no big difference between raw and preprocessed images.

Results show better than random guessing accuracy. Our opinion is that more uniform data set and more complex neural network architecture would provide better results. But experimenting with different network architectures is beyond the scope of this project.

\subsection{Classification Algorithms}

Since this is a classic classification problem, we test some well known machine learning algorithms. Results are show in the figure

\section{Algorithm Comparison}

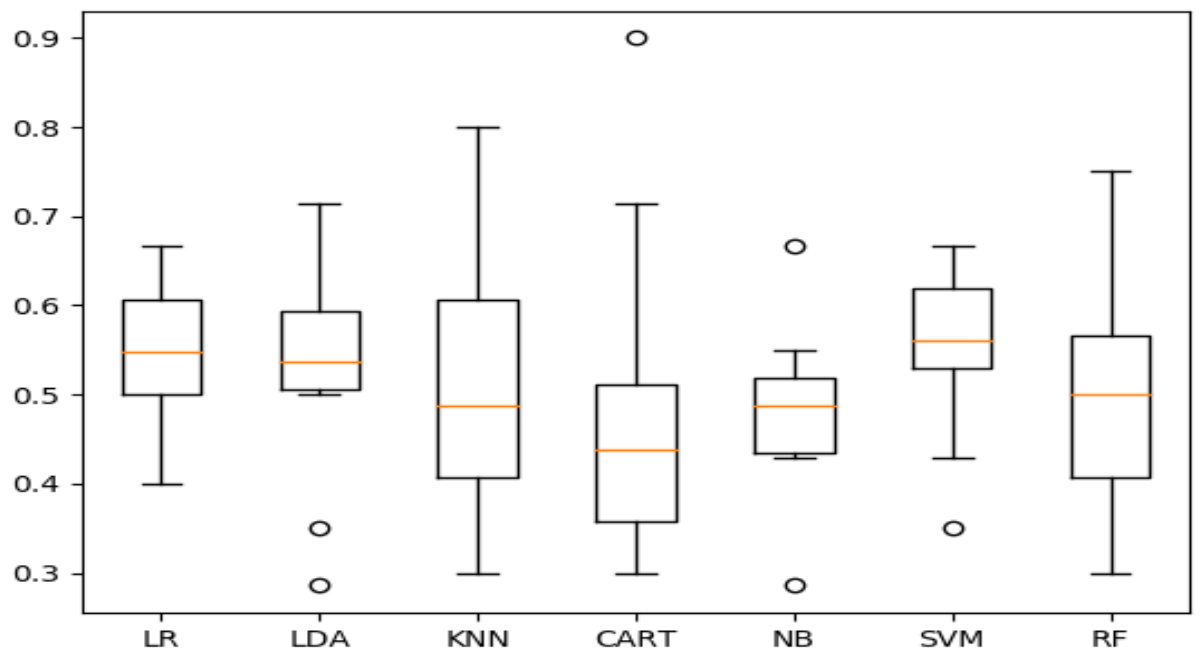

Figure 11: Done multiple of cross validation. Orange lines show average accuracy and boxes' shows average variance. 


\subsubsection{KNN analysis}

We saw that the KNN has the greatest accuracy. We approached the examination of the result for this algorithm. First step was feature selection.

Feature selection

Feature selection is a process where you automatically select those features in your data that contribute most to the prediction variable or output in which you are interested.

Having too many irrelevant features in your data can decrease the accuracy of the models. Three benefits of performing feature selection before modeling your data are:

- Reduces Overfitting: Less redundant data means less opportunity to make decisions based on noise.

- Improves Accuracy: Less misleading data means modeling accuracy improves.

- Reduces Training Time: Less data means that algorithms train faster.

For our features we have following data shown in a figure XXX

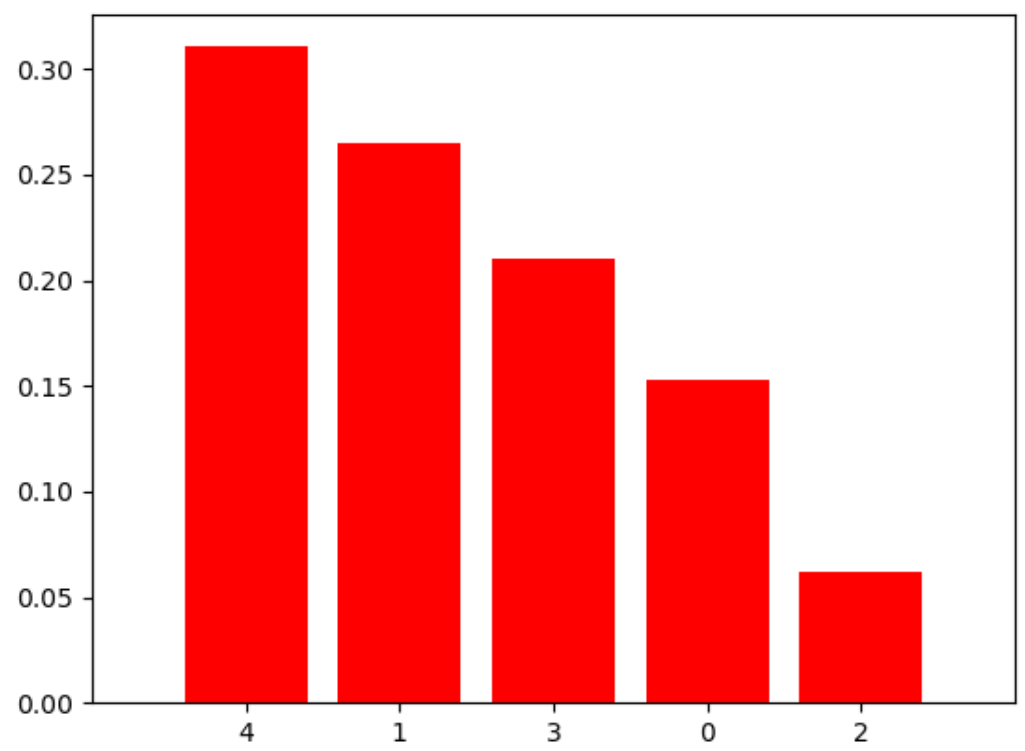

Figure 12Shows feature importance: 1 . feature $4(0.310558) 2$. feature $1(0.264456) 3$. feature 3 $(0.210032) 4$. feature $0(0.152823) 5$. feature $2(0.062130)$

We used 3 most important features to see disposition of organs: 

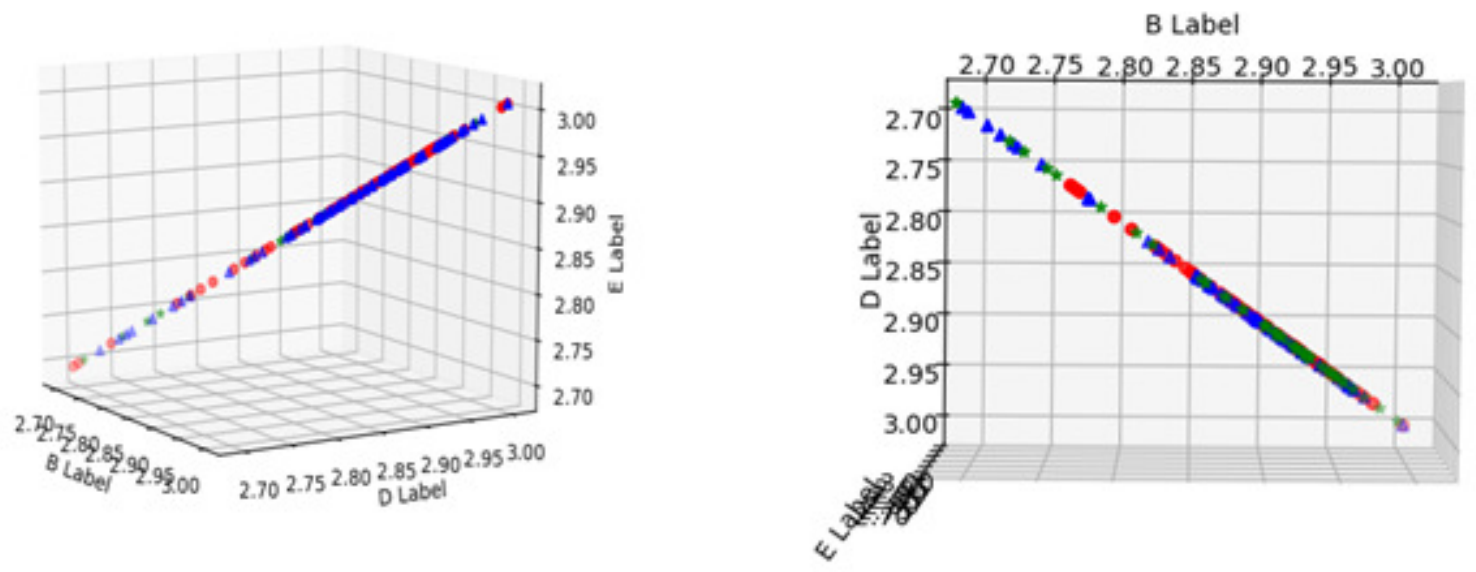

Figure 13: red dots - Breast, blue dots - Kidney, green dots- Lungs. Picture on the right is from the top of diagram.

Algorithm tuning is a final step in the process of applied machine learning before presenting results.

It is sometimes called Hyperparameter optimization where the algorithm parameters are referred to as hyperparameters whereas the coefficients found by the machine learning algorithm itself are referred to as parameters. Optimization suggests the search-nature of the problem.

Phrased as a search problem, you can use different search strategies to find a good and robust parameter or set of parameters for an algorithm on a given problem.

We used the random search as an approach to parameter tuning that will sample algorithm parameters from a random distribution (i.e. uniform) for a fixed number of iterations. A model is constructed and evaluated for each combination of parameters chosen.

As the best parameters we got: $\mathbf{n} \_$neighbors=11 metric=euclidean and results shown in table XXX

Table 1: Shows accuracy of KNN algorithm for each tissue and total.

\begin{tabular}{|l|l|l|l|}
\hline & Total & Correct & Accuracy \\
\hline Lungs & 14 & 7 & $50.00 \%$ \\
\hline Kidney & 14 & 8 & $57.14 \%$ \\
\hline Breast & 14 & 13 & $92.85 \%$ \\
\hline Total & 42 & 28 & $64.29 \%$ \\
\hline
\end{tabular}

\subsubsection{SVC analysis}

This algorithm has the best results in algorithm comparison show in figure XXX We also used random search for algorithm tuning and the best parameters found on development set are: $\mathbf{C}=1000$, kernel=linear. 
Table 2. Shows accuracy of SVC algorithm for each tissue end total

\begin{tabular}{|l|l|l|l|}
\hline & Total & Correct & Accuracy \\
\hline Lungs & 11 & 8 & $72 \%$ \\
\hline Kidney & 9 & 7 & $78 \%$ \\
\hline Breast & 8 & 6 & $75 \%$ \\
\hline Total & 28 & 21 & $75 \%$ \\
\hline
\end{tabular}

\section{ACKNOWLEDGEMENTS}

This work was supported by the Ministry of Education and Science, Republic of Serbia, Science and Technological Development grants III 45005 and TR32037.

\section{REFERENCES}

[1] E. D. P. Z. M. H. D. E. J. M. P. B. M. Pizer, "Contrast Limited Adaptive Histogram Equalization image processing to improve the detection of simulated spiculations in dense mammograms," Journal of Digital Imaging, vol. 193, no. 11, 1998.

[2] R. J. R. P. G. a. H. P. Srivastava, "Enhancement and restoration of microscopic images corrupted with poisson's noise using a nonlinear partial differential equation-based filter," Defence Science Journal, vol. 61, no. 5, p. 452, 2011.

[3] S. Z. B. M. H. E. D. Pisano, "Contrast Limited Adaptive Histogram Equalization image processing to improve the detection of simulated spiculations in dense mammograms," Journal of Digital Imaging, vol. 11, no. 4, pp. 192-200, 1998.

[4] G. Landini, "Fractals in microscopy," Jurnal of Microscopy, vol. 241, no. 1, pp. 1-8, 2011.

[5] F. V. G. G. A. M. V. T. B. G. O. B. M. P. P. W. R. V. V. J. P. A. C. D. B. M. P. M. D. E. Pedregosa, "Scikit-learn: Machine Learning in Python," Journal of Machine Learning Research, vol. 12, pp. 2825-2830, 12102011.

[6] L. B. a. G. L. a. M. B. a. F. P. a. A. M. a. O. G. a. V. N. a. P. P. a. A. G. a. J. G. a. R. L. a. J. V. a. J. a. Bria, "API design for machine learning software: experiences from the scikit-learn project," European Conference on Machine Learning and Principles and Practices of Knowledge Discovery in Databases, pp. 108-122, 2013.

[7] Y. Anzai, Pattern recognition and machine learning, Harcourt Brace Jovanovich, 2012.

[8] L. S. J. T. D. R. K. Jason D. M. Rennie, "Tackling the Poor Assumptions of Naive Bayes Text Classifiers," in ICML, 2013.

[9] L. D.D., "Naive (Bayes) at forty: The independence assumption in information retrieval.," Machine Learning: ECML-98., vol. 1398, pp. 4-15, 1998.

[10] B. G. K. M. S. Mohammad Ahsanullah, Normal and Student's t Distributions and Their Applications, vol. 4, Atlantis Studies in Probability and Statistics, 2014, pp. 7-50. 
[11] A. M. \&. A. K, "OpenCV-Python Documentation", 2017. [Online]. Available: https://media.readthedocs.org/pdf/opencv-python-tutroals/latest/opencv-python-tutroals.pdf. [Accessed 2305 2017].

[12] N. S. Altman, "An Introduction to Kernel and Nearest-Neighbor Nonparametric Regression," The American Statistician, vol. 46, no. 3, pp. 175-185, 2012.

[13] K. E. F. Glenn De'ath, "CLASSIFICATION AND REGRESSION TREES: A POWERFUL YET SIMPLE TECHNIQUE FOR ECOLOGICAL DATA ANALYSIS," Ecology, vol. 81, no. 11, pp. 3178-3192, 2000.

[14] S. Mannel, "Decision Tree Classification of a Forest Using AVIRIS and Multi-Seasonal TM Data," South Dakota School of Mines and Technology, Rapid City, South Dakota, 2012.

[15] R. T. J. F. Trevor Hastie, The Elements of Statistical Learning: Data Mining, Inference, and Prediction, Springer, 2009.

[16] DewiSuryani, S.KOM., M.ENG., "CONVOLUTIONAL NEURAL NETWORK", 2017. [Online]. Available: http://socs.binus.ac.id/2017/02/27/convolutional-neural-network [Accessed 2705 2017].

\section{AUTHORS}

Slađan Kantar was born on 08/12/1993. inKnin, Republic of Croatia. As a young man he came in Aranđelovac, Serbia, where he finished elementary and high school. $\mathrm{He}$ enrolled Faculty of Computer Science, University UNION in Belgrade where he graduated at the Department of Computer Science in 2016. with the topic "Module for automatically testing programming homework solutions (Grader)". He has successfully ends postgraduate studies at the Faculty of Computer Science. From the beginning of the school year 2014/15 he worked as teaching assistant at the Faculty of Computer Science in subject: compiler construction and introduction to computer programming. He is temporary senior software developer in company Moodolar, located in Belgrade.

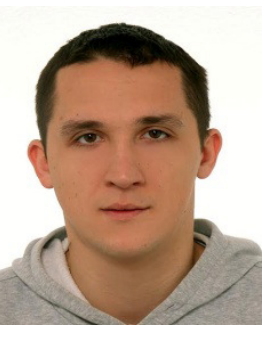

Scientific and professional productionS. Kantar, M. Jovanović, "Implementation of the control algorithm for the 'buggy' mobile robot via application of infrared distance measuring sensors", International Conference on Electrical, Electronic and Computing Engineering, Kladovo, Serbia, June (2017)

Aleksandar Pluškoski was born on 10/12/1993. in Belgrade, Republic of Serbia. Where he was finished elementary and high school. In 2012 he enrolled in The Faculty of Computer Science, University UNION in Belgrade where he graduated at the Department of Computer Science in 2016 with the topic "Alternatives of MS Project, like Project Libre". From the beginning of the school year 2016/2017 he worked as teaching assistance at The Faculty of Computer Science in subject: advance Java programming and web programming. He is successfully finishing postgraduate studies at the Faculty of Computer Science.

Igor Ciganović was born on 08/06/1985. in Dubrovnik, Republic of Croatia. As a young man he came to Pančevo, Serbia, where he finished elementary and high school. He enrolled in Faculty of Computer Science, University UNION in Belgrade where he graduated at the Department of Computer Science in 2016. with the topic "Implemetation of the mechanism for communication between extern service for automatic code grading and Open edX platform and grade calculation". He is successfully finishing postgraduate studies at the Faculty of Computer Science.
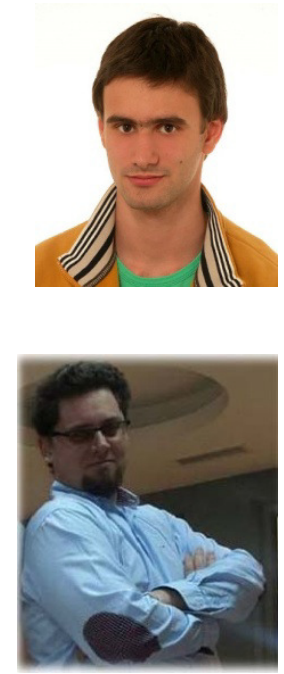
Jelena Vasiljevic works as Science Assistant in Institute Mihajlo Pupin in Belgrade, Serbia and also as Docent for Intelligent Systems teaching on Faculty for Computer Science. She is familiar in teaching Intelligent Systems (Artificial Intelligence), Genetic Algorithms, Neural networks, Fuzzy Logic, Bioinformatics, Knowledge based Systems on $\mathrm{PhD}$, Master and Primary studies. She is a graduate of Faculty for Electrical Engineering on University in Belgrade, Serbia. $\mathrm{PhD}$ research was application of multifractal analysis of digital medical images in cancer diagnostic and for it she has been several times awarded: The First Award on International Medical Fair 2014, The Special Award on International Technical Fair 2014 and many others (also many interviews for TV and newspaper). She is also an author and co-author of many journal and conference peer-reviewed. 


\title{
A COLLABORATIVE CODING FRAMEWORK OF RS AND H.264
}

\author{
Jiejun Hu and Xilong Che
}

Department of Computer Science and Technology, Jilin University, China

\begin{abstract}
The quality of online multimedia is required higher and higher, yet the uncertainty of network, such as interference, connection failure, still exists. In order to ensure the quality of service in multimedia transmission, we incorporate erasure code with video code together, and present a collaborative coding framework of Reed-Solomon code (RS) and H.264. First of all, we analyze the common coding pattern hidden in RS and H.264. Then, we design the collaborative coding framework of the two codes. Finally, we give a prototype implementation and validate the coding effectiveness using standard dataset. The erasure data slice goes through the redundant data tunnel of H.264, thus it will not involve extra traffic overhead. Experimental results show that the framework can increase the quality of media transmission.
\end{abstract}

\section{KEYWORDS}

RS coding, H.264 coding, erasure code, video code, collaborative coding framework

\section{INTRODUCTION}

With the development of the Internet, multimedia has become the common traffic in most networks. However some of the networks are lack of sufficient guarantee of quality-of-service (QoS). Researchers start to focus on new technologies of protecting multimedia data. Comparing to the protection of traditional data, Multimedia data protection has more particular features.

First of all, users can bear the latency of opening a file or picture, but they can't endure the pause while playing a movie. It is hard to balance the data reliability and real-time performance of a large volume of multimedia data. Furthermore, the network environment is dynamic and various. Package loss in multimedia transmission happens frequently, since TCP/IP is a protocol which only makes best efforts.

Video coding technology has been rapidly developed. This paper focuses on the standard video coding framework -- H.264/AVC. H.264 provides many new ways to ensure the quality of the video, because of the best-effort nature of the network. In H.264, it also introduces a concept called redundant slice. Redundant slice is an empty channel for some redundant information of video coding. Mostly the attempt of protecting video data focuses on transmit layer. After the video data is packaged, H.264 uses erasure codes to encode the data packets and produce the redundant data packets, afterwards transmit them into the network.

Natarajan Meghanathan et al. (Eds) : ACSIT, SIPM, FCST, CoNeCo, CMIT - 2017

pp. 57- 70, 2017. (C) CS \& IT-CSCP 2017

DOI : $10.5121 /$ csit.2017.70805 
In this paper, we propose a collaborative coding framework of RS codes and H.264 standard. Different from the previous works which generate the redundant data after video encoding, this paper focuses on the deeper combination of RS and H.264 standard which generates the encoded multimedia data and redundant data as the same time. The contribution of this paper is threefold:

(1) we analyze the features of RS codes and H.264 standard to find the common details of both coding technologies;

(2) we apply the common coding details in RS codes and H.264 to realize the collaborative framework. The collaborative framework can generate both the encoded multimedia data and redundant data when video encoding procedure ends;

(3) we implement the simulation environment to test the framework.

\section{Collaborative FramewOrk For H.264/AVC AND ERASURE CODING}

\subsection{Mechanism Analysis of RS Coding}

In distributed file system, such as Hadoop, erasure code is used in the file system to make sure of the data reliability. The principle of erasure code is firstly coding the blocked data, secondly producing redundant data block. When a client requires data from a distributed system, the system first searches the data. If the data is integrated then the client gets the data. If the system fails to search the integrated data, the system will collect the residual data and the coded redundant data, decode them to recover the original data. Erasure code is a forward error erasure code (FEC code) for binary erasure channel. There are many kinds of erasure codes, for example, Tornado codes, LT codes, Raptor codes and so on. Reed-Solomon code is the most widely used and mature method in erasure codes.

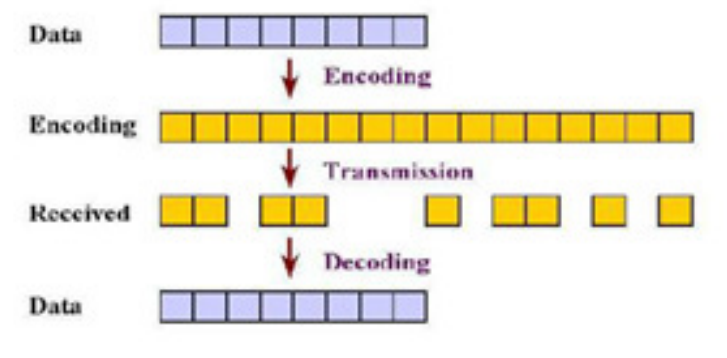

Figure 1. The brief coding procedure of RS codes

In this paper, we introduce Reed-Solomon code into the framework. First of all, we have to declare the principle and procedure of coding. We suppose that there are $\mathrm{n}$ devices, let $\mathrm{D}_{1}, \mathrm{D}_{2} \ldots \mathrm{D}_{\mathrm{n}}$ be the Data Devices(DD), each of them stores $\mathrm{k}$ bytes data. Let $\mathrm{C}_{1}, \mathrm{C}_{2} \ldots \mathrm{Cm}$ be the Redundant Devices (RD), each of them stores ${ }^{k}$ bytes data. The algorithm encodes the DD to get RD. 
The algorithm only proceeds on the word level. So DD must divide into words, and each word contains $\mathrm{w}$ bits. $d_{1}, d_{2} \ldots d n$ is presented the words in one device, so as $c_{1}, c_{2} \ldots c_{m}$. We define the encode function $\mathrm{B}$ :

$$
c_{i}=B_{i}\left(d_{1}, d_{2} \ldots d n\right)
$$

Vector $\mathrm{D}$ and $\mathrm{C}$ represent data words and redundant words. $\mathrm{B}_{\mathrm{i}}$ is used as a row to compose matrix $B$, so matrix $B$ will satisfy the equation $F^{*} D=C$. As RS coding is based on Galois Field, we define $\mathrm{B}$ as Galois Matrix. Vector D and vector $\mathrm{C}$ are composed of a matrix $\mathrm{E}$.

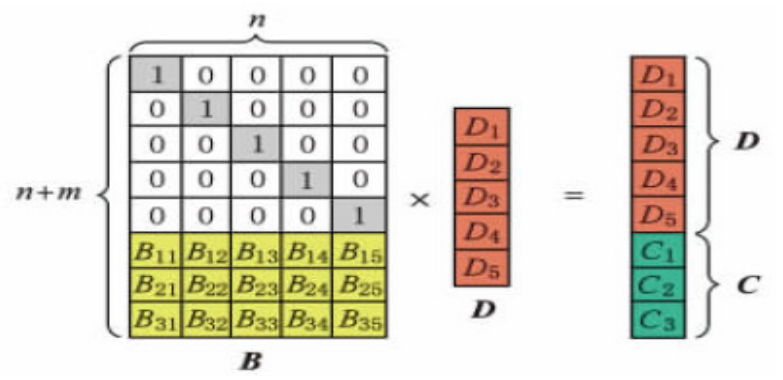

Figure 2. The multiplication of Galois matrix

When one of the devices malfunctions, then we delete the corresponding rows of B and D. As a result of the deleting, it will become a new matrix, and satisfy the equation: $\mathrm{B}^{\prime} \cdot \mathrm{D}^{\prime}=\mathrm{E}^{\prime}$. When $\mathrm{m}$ devices breakdown, there will be a $\mathrm{n} * \mathrm{n}$ matrix, at the same time, any rows in the matrix is linearly independent. Because of the property of the matrix, the value of $\mathrm{D}$ is calculated by Gaussian elimination of $\mathrm{B}^{\prime} \cdot \mathrm{D}=\mathrm{E}^{\prime}$. If the data is totally recovered, the redundant data will be calculated again.

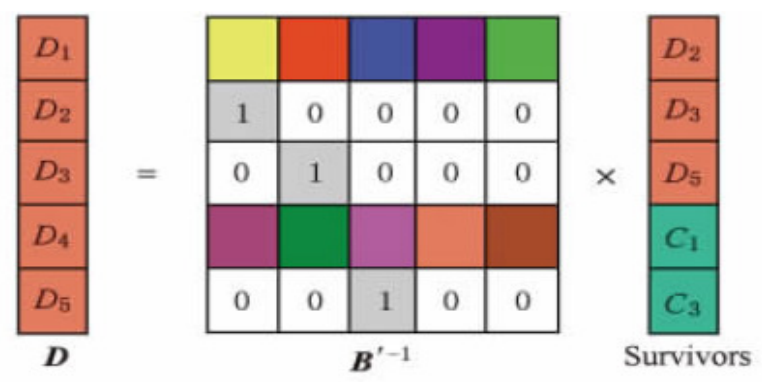

Figure 3. The recovery of the loss data

\subsection{Mechanism analysis of $\mathbf{H} .264$ coding}

In this section, we will discuss the coding details in H.264/AVC and entropy coding.

H.264/AVC provides two parts of the framework, including video coding layer (VCL) and network abstraction layer (NAL). VCL is used for the effective coding of video. At the same time it defines the video data. VCL uses hybrid coding scheme and describes the picture by macroblock. The basic idea of H.264 is motion estimation and time-frequency transform in macroblock. It depends on time and space statistics for residual predicted coding. H.264/AVC contains several important parts, including motion compensation, residual transform coding based on DCT, quantification, zig-zag scan and entropy coding. NAL is used for transmitting the coded data packets into the network. 
H.264 does the coding of $4 \times 4$ residual block in this framework. It provides low computing complexity and high quality of video data by integering transformed the residual block. After transformation and quantification, the video data has some features:

(1) $4 \times 4$ residual block is a sparse matrix;

(2) After zig-zag scanning, the coefficients of DC are non-zero, and most of the coefficients are +1 or -1 ;

(3) The coefficients of the nearby $4 \times 4$ residual blocks are relative;

As the features above, we do the entropy coding after the quantification. There are two methods of entropy coding: CAVLC (Context Adaptive Variable Length Coding) and CABAC (Context Adaptive Binary Arithmetic Coding). CAVLC is less complicated and more efficient than CABAC. Thus we use CAVLC to coding the $4 \times 4$ residual blocks in this paper.

Before entropy coding, the $4 \times 4$ residual blocks should be re-ordered by the zig-zag scan, they are restored into a one-dimensional array, as it shows in Fig. 4. An example for a typical zig-zag scan of quantized transform coefficients could be given as follows, all the coefficients in the matrix are restored in VC_array $=\{0,3,0,1,-1,-1,0,1,0,0, \ldots\}$, as it shows in Fig. 5.

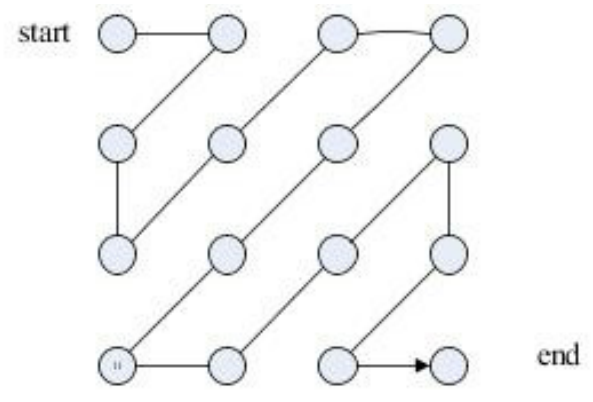

Figure 4. Zig-zag scan

\begin{tabular}{|c|c|c|c|}
\hline 0 & 3 & -1 & 0 \\
\hline 0 & -1 & 1 & 0 \\
\hline 1 & 0 & 0 & 0 \\
\hline 0 & 0 & 0 & 0 \\
\hline
\end{tabular}

Figure 5. A residual block

The simpler entropy coding method uses a single infinite-extent codeword table for all syntax elements except the quantized transform coefficients. Thus, instead of designing a different VLC table for each syntax element, only the mapping to the single codeword table is customized according to the data statistics. The single codeword table chosen is an exp-Golomb code with very simple and regular decoding properties.

In this scheme, VLC tables for various syntax elements are switched depending on already transmitted syntax elements. Since the VLC tables are designed to match the corresponding conditioned statistics, the entropy coding performance is improved in comparison to schemes using a single VLC table.

\subsection{Collaborative coding framework}

In this part we propose the architecture of the collaborative framework of RS codes and H.264 standard. The framework is composed by two main parts: a coding part, which collaborates RS codes and H.264 standard; a transmit part, which transmits redundant data with the coded video data. 


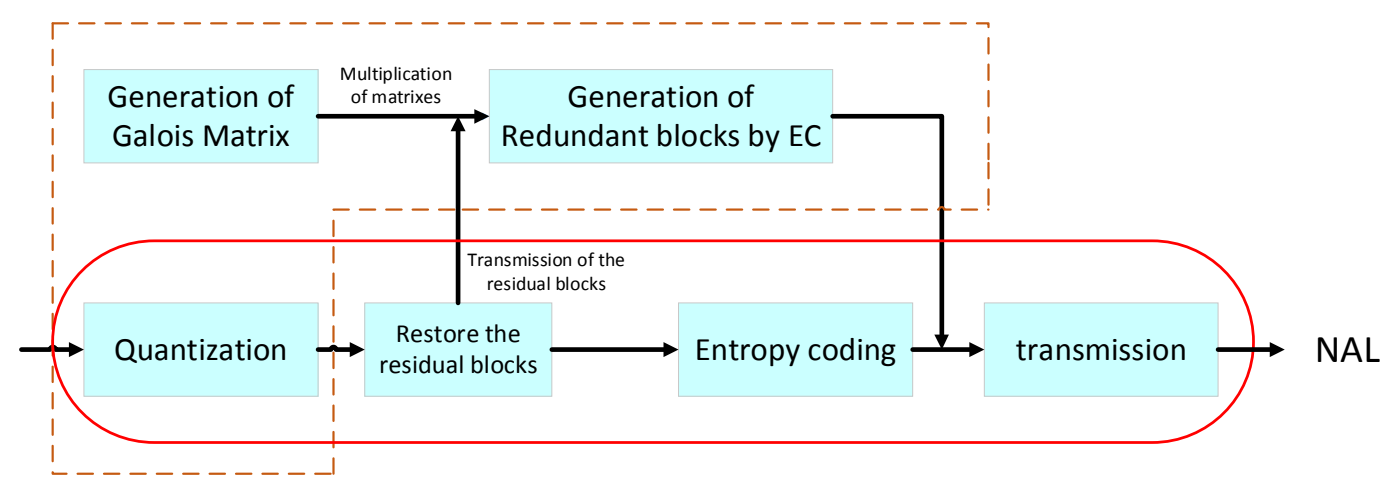

Figure 6. The collaborative framework of RS codes and H.264 standard

In this part we propose the architecture of the collaborative framework of RS codes and H.264 standard. The framework is composed by two main parts: a coding part, which collaborates RS codes and H.264 standard; a transmit part, which transmits redundant data with the coded video data.

For the coding part, the collaborative coding framework begins after the quantization of the $4 \times 4$ residual blocks. We find that entropy coding in H.264 has many things in common with RS coding by analyzing H.264 standard. For example, they both apply algorithms on a matrix. So the $4 \times 4$ residual blocks are suitable for the collaborative coding framework. In the erasure coding part of the whole framework, the first thing to do is to decide the degree of protection of the residual blocks. For example, if the data is important such as an online video meeting, then we have to increase the degree of protection of the data, so as to increase the redundant data. Secondly, according to the degree of protection, RS codes generates Galois matrix. Third, RS codes does the multiplication of Galois matrix and the residual blocks, so we get the redundant data of the video.

For the transmission part, the redundant data is packed into the "similar-NALU". When the coded video data is ready to transmit, then they will transmit with redundant data of the video together. Because the redundant data is different from the original data, we proposed a new structure to transmit the redundant data into the network. We take NALU (the structure for transmitting the packaged video data) of the video data as a reference. As a result, we propose "similar-NALU" to transmit the redundant data. In addition we use redundant slice in H.264 to transmit the "similarNALU", so it won't increase the traffic of the network.

As it shows in Fig. 5, this matrix is the $4 \times 4$ residual block after quantify. We call it residual_block. It is stored in a one-dimension array in zig-zag order, the one-dimension array called VC_array.

We use residual_block to multiply the Galois_matrix. According to the feature of the multiply the matrix, we know that the multiply is between the column vector of the Galois_matrix and the row vector of the residual_block. So when the multiply happens, the resisual_block must be restored in a new one-dimension array in the row order, so that the procedure will end normally. 


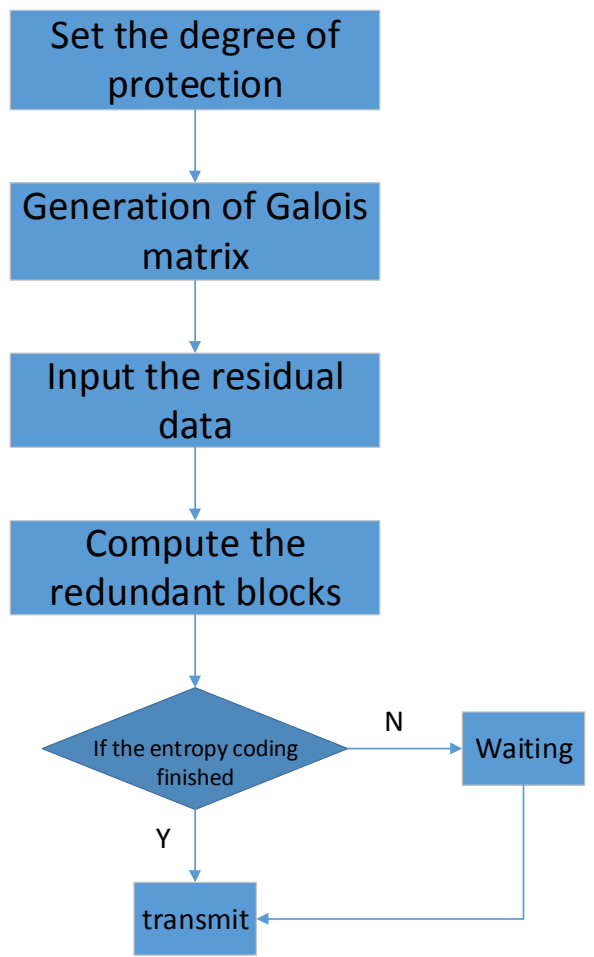

Figure 7. The procedure of RS coding

As it shows in Fig. 5, this matrix is the $4 \times 4$ residual block after quantify. We call it residual_block. It is stored in a one-dimension array in zig-zag order, the one-dimension array called VC_array.

We use residual_block to multiply the Galois_matrix. According to the feature of the multiply the matrix, we know that the multiply is between the column vector of the Galois_matrix and the row vector of the residual_block. So when the multiply happens, the resisual_block must be restored in a new one-dimension array in the row order, so that the procedure will end normally.

In order that our framework will be realized, first we do a little trick of the residual blocks. We divide VC_array into 4 groups, every group has 4 elements. Second we put every group into a new matrix, we call that "new_residual_block", in the row order. As the features of the residual_matrix, the new_residual_block has a new character: the matrix is sparse, even the last few rows are zero. By this way, we make the RS codes and H.264 standard collaborate together. At the same time, we simplified the calculation of the matrix multiplication.

We now give an example of the collaborative coding framework. The residual matrix shows in Fig. 5. First, it is scanned by the zig-zag order, so we get a sequence like: $0,3,0,1,-1,-1,0,1,0$, $0, \ldots$ Second we store the elements into the VC_array $=\{0,3,0,1,-1,-1,0,1,0,0, \ldots\}$. As what we talked above, the new_residual_matrix shows in the Fig. 8. Then the Galois_matrix and new_residual_block do the multiplication . 


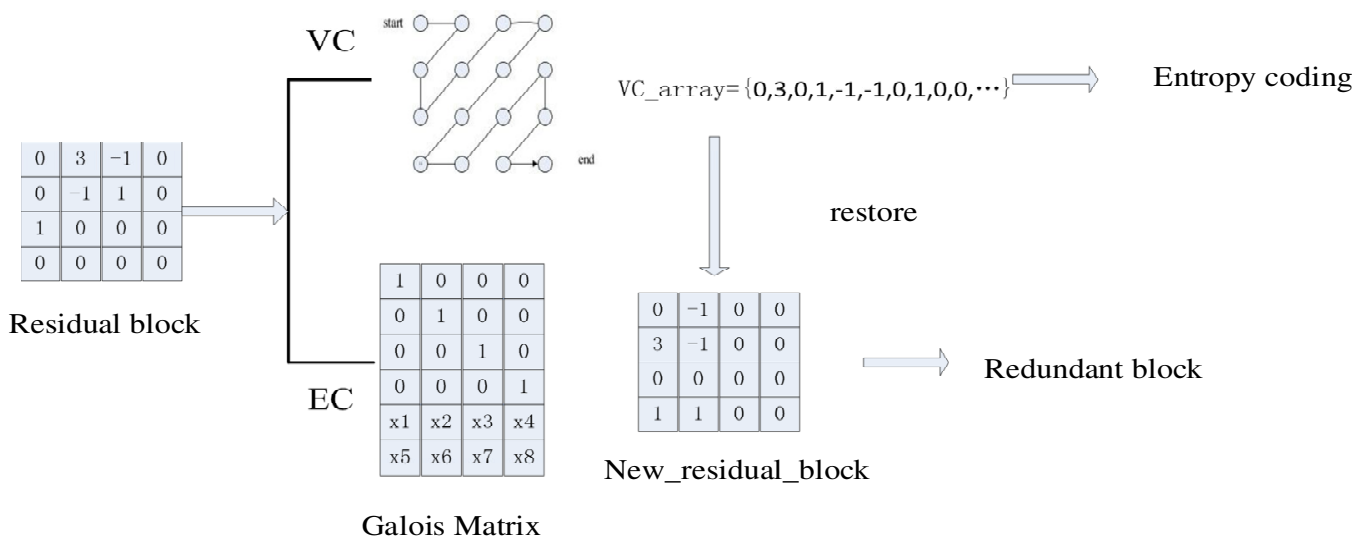

Figure 8. The example of the collaboration coding framework

Although the character of the new_residual_block increases the efficiency of the coding, it can't recover the real residual block. So we introduce the procedure called "inverse zig-zag scan" to recover the original video data, as it shows in Fig. 9.

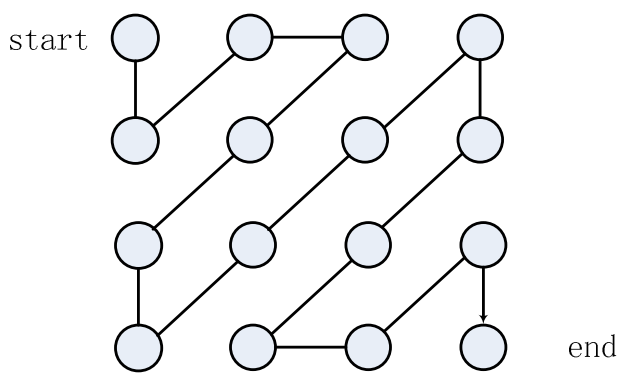

Figure 9. Inverse zig-zag scan

After the collaborative coding framework, all the data blocks are ready to transmit into the network. Here the problem comes, H.264 has already had a structure to pack the video data block, it uses a structure called NALU to realize this procedure. What to do with the redundant data coded by erasure codes? We designed a new structure called "similar-NALU" to pack the redundant data. Similar-NALU is a twin of the original NALU of H.264.

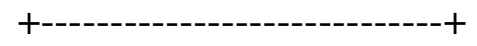

$10|1| 2|3| 4|5| 6|7|$

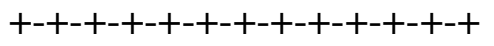

|MI IM | E_TYPE |

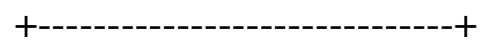

Figure 10. The structure of similar-NALU 
In similar-NALU, there are three fields:

(1) M(Mark): this is the flag of a similar-NALU, because the flag of NALU normally sets "0", so we use " 1 " to represent similar-NALU.

(2) IM(IMportant): IM represents the importance of the similar-NALU. Usually we set " 00 ".

(3) E_TYPE(erasure_type): E_TYPE is the type of the erasure coded data, it has three kinds of data: $Y$ data, $U$ data and $V$ data, according to the video data types.

There is a function called "redundant slice" in H.264. This function uses residual bandwidth of video data to transmit the redundant data. However, redundant slice is not really realized. So in our work, we use the residual bandwidth of the redundant slice to transmit the similar-NALU. In this way, on one hand we increase the reliability of the video data, on the other hand, the redundant data doesn't increase any extra bandwidth.

Now we come to the last part of our collaborative framework: the decode procedure. Firstly, we need to check if the NALUs of the video is integral. If so, the video data applies for the decode procedure of H.264. If not, the framework uses the redundant data to recover the original data. Using RS codes to recover the original data as follows:

(1) Generate the corresponding Galois_matrix for the redundant data;

(2) Use Berlekamp-Massey algorithm and Forney algorithm to estimate the location of the errors;

(3) Recover the error elements.

Once we get the original NALU, we use H.264 to decode the video data.

\section{IMPLEMENTATION AND EVALUATION}

\subsection{Implementation}

In order to implement the collaborative coding framework, we extend the JM(Joint Model, version 13.2) and apply for RS codes in to JM framework. The combination of two coding schemes is after quantization. The collaborative coding framework is realized in C. We also make use of the "foreman_part_qcif.yuv" data set, which is a standard testing data set. In H.264, we apply the baseline mode of coding to the video data.

We also implemented a simulator on NIST Net to evaluate the collaborative framework's performance in different network conditions. NIST Net is a tool to facilitate testing and experimentation with network code through emulation. The version of NIST Net is $2.0 .12 \mathrm{~b}$. It works on the Linux OS, it acts like a black box. We can use NIST sNet to simulate many kinds of network conditions, such as: packet loss, delay, jitter, congestion and limited bandwidth. In our framework, we only realize the packet loss function.

The simulation of the network including 3 parts:

(1) A sender which runs the collaborative framework to encode;

(2) A router which is a virtual machine runs NIST Net, and the router must have eth0 and eth1 to communicate with the sender and the receiver;

(3) A receiver which also runs the collaborative framework to decode. 
The topology is as the following picture shows:

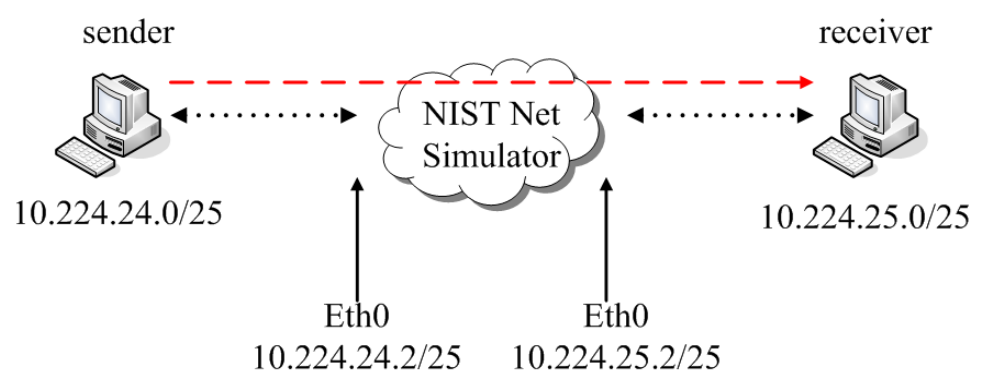

Figure 11. The topology of the simulation

In addition, Gilbert-Eliott model(GE model) is introduced into NIST Net simulator. In GE model, the channel is classified into two states: "good" and "bad". We donate $\mathrm{P}_{\mathrm{G}}$ as the possibility of good state and $\mathrm{P}_{\mathrm{B}}$ as the possibility of bad state. The possibility of changing good state to bad state is $\mathrm{P}_{\mathrm{GB}}$, and $\mathrm{P}_{\mathrm{BG}}$ is the opposite. Suppose the channel is in steady state, then:

The average loss possibility of the whole EB model:

$$
\mathrm{P}_{\text {average }}=\mathrm{P}_{\mathrm{G}} * \mathrm{P}_{\mathrm{BG}} /\left(\mathrm{P}_{\mathrm{BG}}+\mathrm{P}_{\mathrm{GB}}\right)+\mathrm{P}_{\mathrm{B}} * \mathrm{P}_{\mathrm{GB}} /\left(\mathrm{P}_{\mathrm{BG}}+\mathrm{P}_{\mathrm{GB}}\right)
$$

the state shows in the figure :

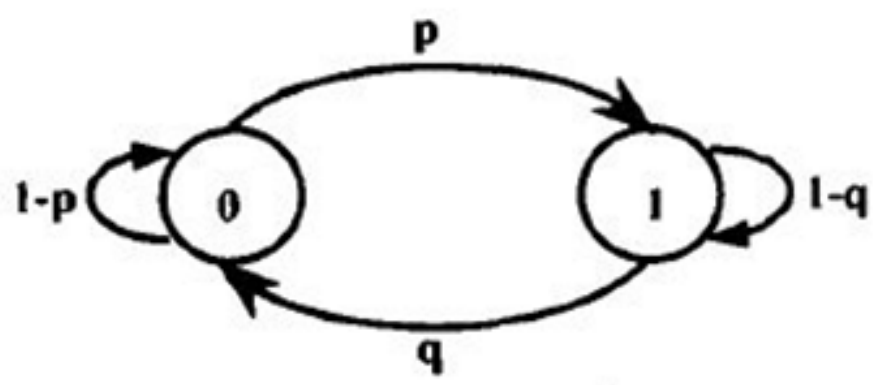

Figure 12. GE Model

By adjusting the parameters of the model, the NIST Net simulates a network condition with 5\%, $10 \%$ and $15 \%$ packet loss.

\subsection{Evaluation}

This section describes the evaluation of the collaborative framework using NIST Net simulator. Generally, Signal-to-noise ratio (abbreviated SNR) is a measure used in science and engineering that compares the level of a desired signal to the level of background noise. The definition of SNR as follows:

$$
\text { SNR }=10 * \log _{10} \frac{\text { Signal_power }}{\text { Noise_power }}
$$


When the typical reference threshold value of SNR is $25-55 \mathrm{db}$, the noise of the picture is low and the quality is good. When SNR is 60db, the picture is the best and without noise.

We focus this evaluation on three aspects.

(1) Without this collaborative framework, what will the video quality be when there is packet loss?

The first experiment we test is only the H.264 standard in different network condition. The purpose of the first experiment is to see how these parameters change during the different packet loss rate. We use 3 different packet loss rates to test. Figure 6.7 shows the SNR of Ydata, Udata and Vdata in video data. As the increasing of the packet loss rate, SNRs decrease.

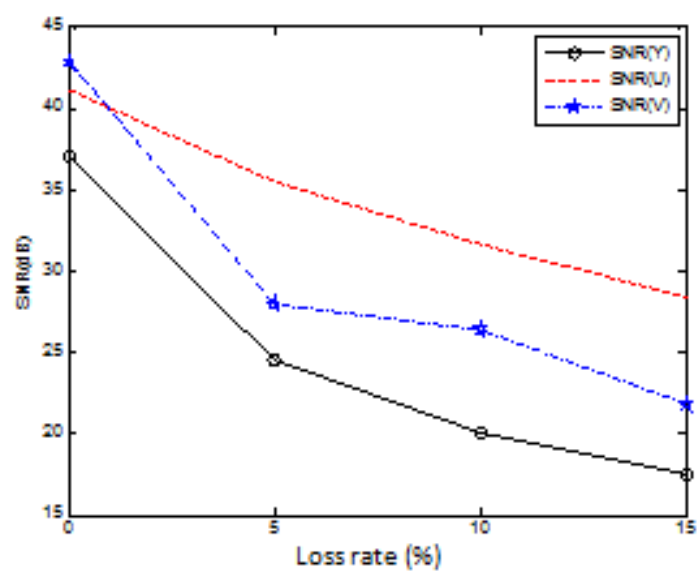

Figure 13. SNR under different data loss rates witåhout collaborative framework

After knowing the result of the first experiment, we want to find out if our collaborative framework help or not. Thus here comes the second experiment.

(2) Does the collaborative framework help when the packet loss rate increases?

The purpose of the second experiment is to show that the collaborative framework does make an effort in a poor network condition. In this experiment, the erasure codes set as $\operatorname{RS}(15,9)$, that means after the RS encoding there will be 15 data blocks, and there are 9 original data blocks, so the redundant data block is 6 . At the same time, these 6 redundant data blocks use the "redundant slice" to transmit into the network. Figure 6.8 shows the effects of the collaborative framework. When the packet loss rate increases, the SNR is increased by the collaborative framework nearly $1 \mathrm{db}$. 


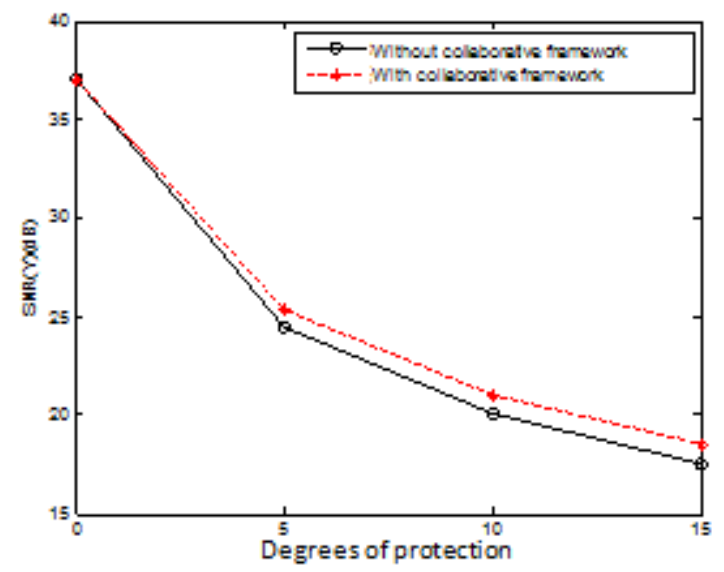

Figure 14. SNR under different data loss rates with collaborative framework

The result shows that the framework helps when the packet loss rate increases. This result turns out that the collaborative framework of RS codes and H.264 standard can do as a supplement to ensure the quality of the video. With the third experiment we will adjust the parameters of the framework to see the result.

(3) How will the quality of the video change when we modify the parameters in the collaborative framework?

In the third experiment, we adjust the parameters of the RS code in the collaborative framework with the same bad network conditions -- 5\% packet loss. We apply $\operatorname{RS}(15.9), \operatorname{RS}(15,7)$ and $\mathrm{RS}(15,5)$. We create more redundant data blocks in the encoding procedure. In figure 6.9, we find that with the increasing of the redundant data blocks, the SNR increases nearly $2 \mathrm{db}$.

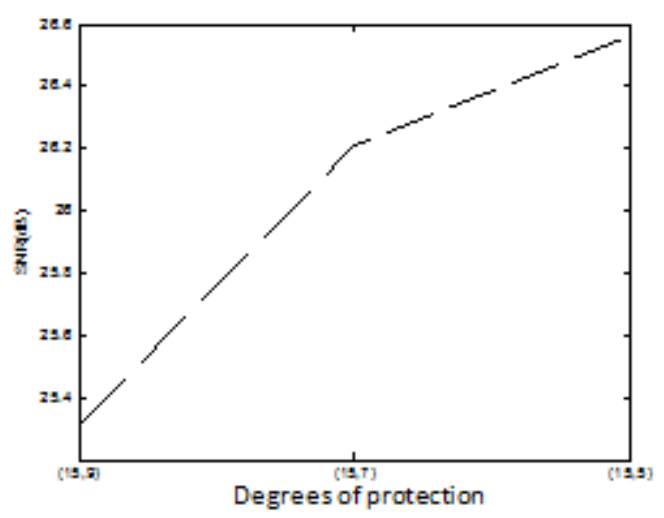

Figure 15. SNR under different degrees of protection with collaborative framework

\section{RELATED WORKS}

We have talked about all the details and the result of the simulation and experiments. There are two special aspects of the collaborative framework of RS codes and H.264 standard. On one hand, the whole collaborative framework makes two coding schemes together and achieves the 
quality of the video. On the other hand "similar-NALU" makes it possible to avoid increasing any traffic while transmitting the redundant data. Related work falls into three aspects: using erasure coding in the transmitting stage, analyzing the importance of the video data and then using erasure codes and using different erasure codes in transmitting stage.

Xingjun Zhang et al. proposed a method called ULP-Unequal Loss Protection scheme for data transmission over lousy networks. This is developed from the ELP - Equal Loss Protection. By analyzing the scheme of H.264/AVC, they found out that the packet transmitting in network has unequal importance. They classify the data into three types - data partition A, B, C. In decoding, PA is the most important data partition of these three, and then PB, PC. And they apply ReedSolomon code with different recoverability to the data partitions. The result shows that at the same loss rate, the video quality of ULP is much better than ELP.

R.Razavi et al. proposed a method that using growth codes to protect data partitions of H.264 video streaming. Using a growth codes was especially for the wireless networks. The method provided a hierarchy of protection, both for I frames and P frames. They used Raptor channel coding which allowed incremental protection of video data partitions in network. It also used UEP channel coding. By Raptor codes, this method of protecting data was up to $10 \mathrm{db}$ in PSNR(video quality parameter). Different from these two works above, our work pay attention to the common coding scheme between the RS codes and H.264 standard.

Yevs Dhondt et al. proposed a flexible macroblock scheme for unequal error protection. In H.264/AVC, Flexible Macroblock Ordering (FMO) is a new tool. This paper improved the original FMO. The importance of macroblock will be recalculated and re-packed in a slice. By this way, it has achieve an increase of PSNR of $4 \mathrm{~dB}$ on average.

Many researchers pay most attention on the phase, which the video has been coded by H.264 and ready to transmit to the channel. By using this way, they achieve the better quality of the video data. However the time efficiency of erasure coding is low. At the same time, the video data they used to do the erasure coding is in large granularity, it can't use the redundant slice to transmit, so it increases the redundant data in the channel. In our work, firstly it provides a framework that makes the erasure coding procedure "go-through" the video coding under H.264. Secondly we produce the smaller granularity redundant data to recover the lost data, the decoding procedure will be simple. Finally we use the redundant slice to transmit the redundant data, and pack the redundant data into the similar-NALU. These all makes our work different from the former ones.

\section{CONCLUSION}

This research proposed a collaborative coding framework of RS code and H.264 code. Our efforts include three main parts. First, we analyzed the coding mechanism of RS code and H.264 code, and found out that both coding schemes are based on using of media matrix, which stands as the basis for collaborative coding. Second referencing to the traditional NALU, we gave the twin NALU called "similar-NAUL" to transmit the redundant data with the original bandwidth. Finally we simulated the package loss codition in network environment and validated the effective net of collaborative coding framework proposed. Experimental results show that the combination of erasure coding and video coding did improve the value of SNR. 


\section{ACKNOWLEDGEMENT}

This work is funded by: European Framework program(FP7) under Grant No. FP7-PEOPLE2011-IRSES, and by National Natural Science Foundation of China under Grant No. 61073009, and by National Sci-Tech Support Plan of China under Grant No. 2014BAH02F03.

\section{REFERENCE}

[1] Karp R, Luby M, Shokrollahi A. Finite length analysis of LT codes[C]//Information Theory, 2004. ISIT 2004. Proceedings. International Symposium on. IEEE, 2004: 39

[2] Xianghong L, Jiwu S. Summary of research for erasure code in storage system[J]. Journal of Computer Research and Development, 2012, 49(1): 1-11..

[3] Wang Y, Zhu Q F. Error control and concealment for video communication: A review[J]. Proceedings of the IEEE, 1998, 86(5): 974-997.

[4] Wiegand T, Sullivan G J, Bjontegaard G, et al. Overview of the H. 264/AVC video coding standard[J]. Circuits and Systems for Video Technology, IEEE Transactions on, 2003, 13(7): 560576.

[5] Wang Y, Ostermann J, Zhang Y Q. Video processing and communications[M]. Upper Saddle River: Prentice Hall, 2002.

[6] Dhondt Y, Lambert P, Van de Walle R. A flexible macroblock scheme for unequal error protection[C]//Image Processing, 2006 IEEE International Conference on. IEEE, 2006: 829-832.

[7] Zhang X, Peng X, Fowler S, et al. Robust H. 264/AVC video transmission using data partitioning and unequal loss protection[C]//Computer and Information Technology (CIT), 2010 IEEE 10th International Conference on. IEEE, 2010: 2471-2477.

[8] Razavi R, Fleury M, Altaf M, et al. H. 264 video streaming with data-partitioning and growth codes[C]//Image Processing (ICIP), 2009 16th IEEE International Conference on. IEEE, 2009: 909912.

[9] Schierl T, Schwarz H, Marpe D, et al. Wireless broadcasting using the scalable extension of H. 264/AVC[C]//Multimedia and Expo, 2005. ICME 2005. IEEE International Conference on. IEEE, 2005: 884-887.

[10] Lin W K, Chiu D M, Lee Y B. Erasure Code Replication Revisited[C]//Peer-to-Peer Computing. 2004: 90-97.

[11] Mohr A E, Riskin E A, Ladner R E. Unequal loss protection: Graceful degradation of image quality over packet erasure channels through forward error correction[J]. Selected Areas in Communications, IEEE Journal on, 2000, 18(6): 819-828.

[12] Girod B, Aaron A M, Rane S, et al. Distributed video coding[J]. Proceedings of the IEEE, 2005, 93(1): 71-83. 


\section{AUTHORS}

Jiejun Hu, received her master degree in Computer Science from Jilin University in 2012. Currently she is a PhD student in Jilin University, China. Her current research areas are computer network, future network and technology, resource allocation in network, and related applications.

Xilong Che received his MEng and $\mathrm{PhD}$ degrees in Computer Science from Jilin University in 2006 and 2009. Currently, he is a lecturer at the College of Computer Science and Technology, Jilin University, China. His current research areas are parallel and distributed computing, machine learning, and related applications. He is a member of the IEEE.
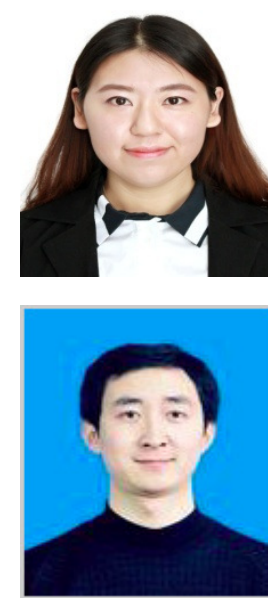


\title{
BUILDING A TEXT-TO-SPEECH SYSTEM FOR PUNJABI LANGUAGE
}

\author{
Rupinderdeep Kaur, Mr. R.K. Sharma and Mr. Parteek Kumar \\ Computer Science and Engineering department, \\ Thapar University, Patiala, Punjab
}

\begin{abstract}
A Text-To-Speech (TTS) system is a computer application that is capable of converting typed text into speech. This paper contains description of working of a TTS system along with architecture of the system and various available TTS systems for Indian Languages with comparison of these systems on the basis of the methods used by them for speech synthesis. TTS system generally involves two steps, text processing and speech generation. Synthetic speech may be used in several applications, like, telecommunications services, language education, aid to handicapped persons, fundamental and applied research etc. TTS involves many challenges during the process of conversion of text to speech. The most important qualities expected from speech synthesis system are naturalness and intelligibility. The general architecture of a TTS system and different waveform generation methods are discussed in this paper. A scheme for developing a TTS system for Punjabi Language is also included in this paper.
\end{abstract}

\section{KEYWORDS}

TTS, Phonetics, Prosody, Speech Synthesis

\section{INTRODUCTION}

Spoken words (speech) play a great role in the lives of people. Speech represents the spoken form of a language and is also one of the important means of communication. Over the past few decades, a good amount of research is being done in the field of converting text into speech. These efforts have resulted in important advances with many systems being able to generate the sound close to a real natural sound. These advances in speech synthesis also pave the way for many new speech related applications. Some of these applications are audio books, announcement systems, speech systems for visually handicapped etc.

\section{TEXT-TO-SPEECH SYSTEM}

The function of a Text-To-Speech (TTS) system is to convert an arbitrary text to a spoken waveform. This broadly involves two steps, namely, text processing and speech generation. Text processing is used to convert the given text to a sequence of synthesis units, while speech generation includes the generation of an acoustic wave form corresponding to each of these units in the sequence [1] [2].

Natarajan Meghanathan et al. (Eds) : ACSIT, SIPM, FCST, CoNeCo, CMIT - 2017 


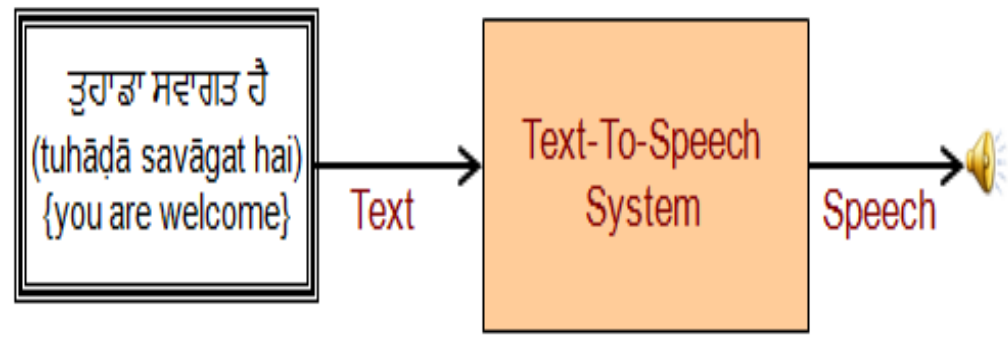

Figure 1. Text-To-Speech System

\section{OBJECTIVES OF TEXT-TO-SPEECH SYSTEM}

People are very sensitive, not just to the words that are spoken, but also to the way these are spoken. So the primary and the most important objective of any TTS system should be to produce a sound which looks like a natural sound. The sound should not be felt highly mechanical and should be comforting when listened. The important qualities of a speech synthesis system are naturalness and intelligibility. Naturalness means how close the output sounds is to human speech, while intelligibility is the ease with which the output is understood by a listener. One more important objective of a TTS system is that it should be able to take any written input, i.e., any format or font and converts this to speech.

\section{THE BASIC TERMINOLOGY}

The basic terms used in speech synthesis include Phonology, Phonemics, Phonetics and Prosody. These are briefly explained in following sub sections.

\subsection{Phonology}

Phonology is the branch of linguistics that deals with systematic organization of sounds in a language. It has traditionally focused on the study of systems of phonemes in languages. It may also cover a linguistic analysis either at a level beneath the word or at all levels of language where sound is considered to be structured for conveying linguistic meaning [22]. This phoneme system and linguistic analysis is a key process for developing a TTS system for the language under consideration. Analysis of sounds for a given language helps in developing an efficient TTS system for that language.

\subsection{Phonemics}

A phoneme is a basic unit of a language's phonology, which is combined with other phonemes to form meaningful units such as words or morphemes. Phoneme can be described as "the smallest contrastive linguistic unit which may bring about a change of meaning"[22]. For example, the words घय (bāp) /baap/ \{father\}, या (pāp) /paap/ \{sin\} and भग्य (māp)/maap/ \{measure $\}$ can be obtained by replacing the phoneme $/ \mathrm{p} /$ for the phoneme $/ \mathrm{m} /$ and $/ \mathrm{b} /$. These words, which differ in meaning through a contrast of a single phoneme, are called minimal pairs. In order to build an efficient TTS system, a deep study of these phonemes is very necessary. 


\subsection{Phonetics}

Phonetics is the branch of linguistics that comprises the study of sounds of human speech [22]. The International Phonetic Alphabet (IPA) is used as the basis for the phonetic transcription of speech. It is based on Latin alphabet and is able to transcribe most features of speech such as consonants, vowels, and suprasegmental features. Every documented phoneme available within the known languages in the world is assigned its own corresponding symbol. Figure 2 contains the IPA chart given by International Phonetics Association. According to the IPA symbols

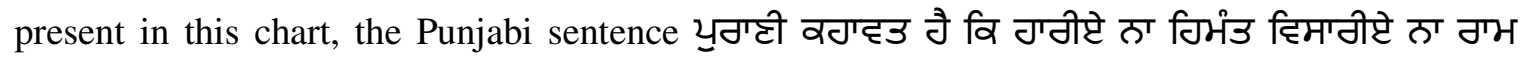
(purāṇ̄ kahāvat hai ki hārīē nā himant visārīē nā rām) can be transcribed as porá:niikəhá:və_thæ̀_ke:_há:riená:hımə_tă_bısa:ciynárà:m.

It is worth mentioning here that phonetics removes the language barrier and converts the set of phonemes to corresponding phonetic symbols. Phonetics plays major roles in TTS system because it not just concatenates the symbols to form a word, as done in phonology, but also represents the symbols for supra-segmental and tonal features of human sounds. For languages which are highly a tonal language, after phonology, conversion of phonemes to phonetics is very necessary for TTS system to be natural.

\subsection{Prosody}

In linguistics, Prosody is the rhythm, stress, and intonation of speech. Prosody may reflect various features of the speaker or the utterance: the emotional state of the speaker; the form of the utterance (statement, question, or command); the presence of emphasis, contrast, and focus. Prosodic features are supra-segmental. They are not confined to any one segment, but occur in some higher level of an utterance. Prosodic units are marked by phonetic cues, such as a coherent pitch contour - or the gradual decline in pitch and lengthening of vowels over the duration of the unit, until the pitch and speed are reset to begin the next unit. Breathing, both inhalation and exhalation, seems to occur only at these boundaries where the prosody resets [22]. Phonetics helps to incorporate these prosodic features into a TTS system to make it natural and intelligent.

\section{ARCHITECTURE OF TEXT-TO-SPEECH SYSTEM}

The conversion of Text-To-Speech is not a single step process. Many things need to be considered in the process of conversion of Text-To-Speech. The process of converting text into speech breaks down into a number of stages. The general architecture of converting Text-ToSpeech is shown in Figure 3. O Malley [3] described the process of converting text into speech as a four stage process. These stages are:

- Text Pre-processing and Text Normalization

- Linguistic Analysis

- Prosodic Prediction

- Waveform Generation 
THE INTERNATIONAL PHONETIC ALPHABET (revised to 2005)

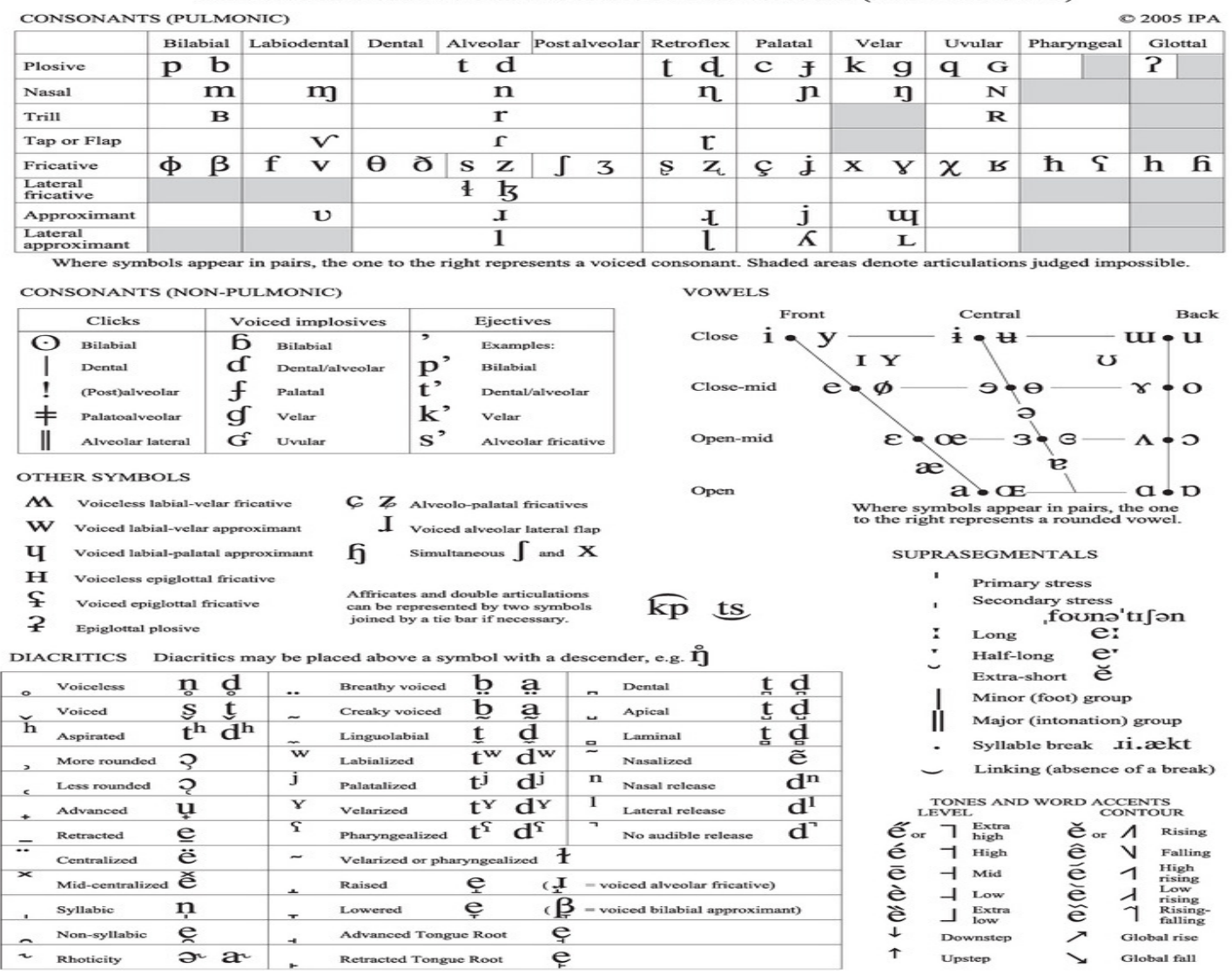

Figure 2. IPA Chart

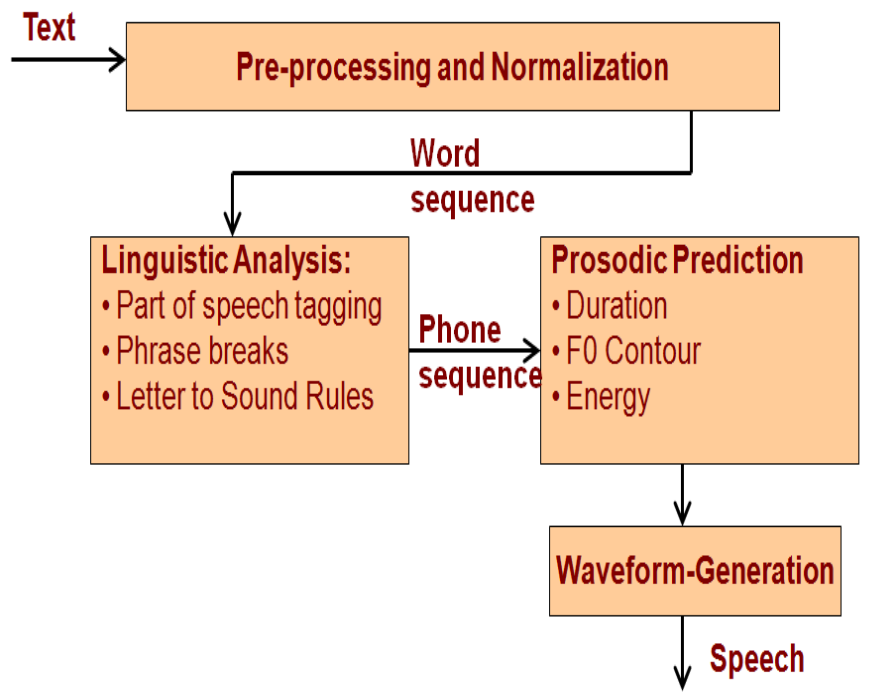

Figure 3. Architecture of Text-To-Speech System 


\subsection{Text Pre-Processing and Text Normalization}

The typical input to a Text-To-Speech system is the text as available in electronic documents, news papers, blogs, emails etc. The text available in real world is a sequence of words available in standard dictionary. The text contains several non-standard words such as numbers, abbreviations, homographs and symbols built using punctuation characters such as exclamation '!', smileys ':-)' etc. The goal of text pre-processing module is to process the input text and generate appropriate phone sequences for each of the words.

The text in real world consists of words whose pronunciation is typically not found in dictionaries or lexicons such as "मेभ", "भंगळ", and "उా." etc. Such words are referred to as Non-Standard Words (NSW). Various categories of NSW are as follows:

- Numbers, whose pronunciation changes depending on whether they refer to currency, time, telephone numbers, zip code etc.

- Abbreviations, contractions, acronyms such as "उా.", "सैढटी.", "D.." etc.

- Punctuations 3-4, +/-, and/or, 4), dates, time, units and URLs.

The other issue of NSW is that, most of NSWs are homographs, i.e., words with same written form but different pronunciation. For example, IV which can vary as "four" in (भग्ठटीवल्डIV), the "fourth" in (ठठठठIV), and in the same as three or four digit numbers which could be dates and ordinary numbers (26 नठहठी 1950, 1950, 50 टठ).

Machine learning models such as Classification and Regression Trees (CART) have been used to predict the class of NSW which is typically followed by rules to generate appropriate expansion of a NSW into a standard form [4]. Rule-based systems for NSW operate on local grammars containing abstract contexts for within-sentence occurrences and sentence boundaries [28]. Mikheev's rule-based segmentation [29] is preceded by capitalized word disambiguation. There are also some language modelling and machine learning approaches for normalization subtasks. For example, in Sproat et al., [4] word normalization is amongst others formulated in terms of maximizing the conditional probability of a normalized word sequence given an observed token sequence.

\subsection{Linguistic Analysis}

Linguistic analysis refers to scientific analysis of a language sample. It involves at least one of the five main branches of linguistics, which are, phonology, morphology, syntax, semantics, and pragmatics. Linguistic analysis can be used to describe unconscious rules and processes that speakers of a language use to create spoken or written language, and this can be useful to those who want to learn a language or translate from one language to another. For TTS system, linguistic analysis is done by part of speech tagging, prosodic phrase break marking and by defining letter to sound rules.

\subsubsection{Part of Speech tagging}

It is a process of assigning a part-of-speech to each word in a sentence. Part-of-Speech (POS) tagging is the task of determining the correct parts of speech for a sequence of words. Words are 
often ambiguous with respect to part of speech. The word हाठ (waar), e.g., is a noun in the sentence "ठग ठे माभ उे हाव वीउा" (ram ne sham te waar kita), a finite verb in the sentence "ठग्भ ठे

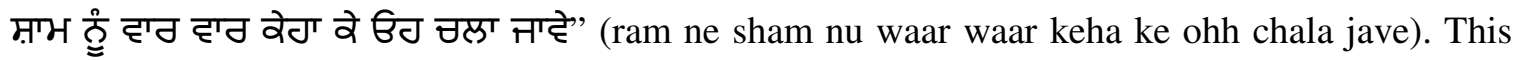
ambiguity is tackled by POS and results produced with predefined tags.

Rule-based approaches like ENGTWOL [30] operate on dictionaries containing word forms together with associated POS labels and morphologic and syntactical features and context sensitive rules to choose appropriate labels during application. In statistical approaches [31], the most probable tag sequence given the observed word sequence is generally estimated. In transformation-based tagging [32] a hybrid approach can be found, where disambiguation rules are derived by statistical means.

\subsubsection{Phrase breaks}

Phrase breaks incorporate the silence region in a speech. In text, it is marked with the help of POS. For Example consider the Punjabi sentence given in (1) and (2).

$$
\begin{aligned}
& \text { भंउठी ती के विण वि मॅत माइे ऐेम़.... } \\
& \text { (mantrī jī nē kihā ki ajj sāḍē dēsh) } \\
& \text { भंउठी ती ठे विण वि [pause] भॅत माइे टेम.... } \\
& \text { (mantrī jī nē kihā ki [pause] ajj sāọē dēsh) }
\end{aligned}
$$

Here in (1), 'वि' is the preposition marked by POS and (2) tells about a phrase break, i.e., while converting this text into speech, there will be a small pause in this region [27].

\subsubsection{Letter to sound rules}

Text to phoneme conversion is to derive the pronunciation for each word based on its spelling. Word pronunciations may be conveniently described as sequences of phonemes, which are units of sound in a language that serve to distinguish one word from another. There are mainly two

approaches for text to phoneme conversion. These are dictionary based approach and rule based approach.

In Dictionary based approach, a large dictionary containing all the words of a language and their correct pronunciations is stored in the database. To determine the correct pronunciation of each word, it is looked up in the dictionary and the spelling is replaced with the pronunciation specified in the dictionary. This approach is quick and accurate, but it fails if looked up word is not present in the dictionary. Also, the size of dictionary should be considered carefully, as the memory space requirements of the synthesis system depends on the size[7].

In Rule based approach, pronunciation rules are applied to words, to determine their pronunciations based on their spellings. This approach is useful for the languages having very regular writing system, therefore, the prediction of the pronunciation of words based on their spellings is quite successful. In contrast, this approach works on any input, but the complexity of the rules grows substantially as the system takes into account irregular spellings or pronunciations [11]. 


\subsection{Prosody Prediction}

Prosodic prediction analysis deals with modeling and generation of appropriate duration and intonation contours for the given text. This is inherently difficult since prosody is absent in text. For example, the sentences - where are you going?; where are you GOING? and where are YOU going?, have same text-content but can be uttered with different intonation and duration to convey different meanings. To predict appropriate duration and intonation, the input text needs to be analyzed. This can be performed by a variety of algorithms including simple rules, example-based techniques and machine learning algorithms. The generated duration and intonation contour can be used to manipulate the context-insensitive diphones in diphone based synthesis or to select an appropriate unit in unit selection voices [21]. The prosody can be predicted by duration, F0 contour and energy contour.

\subsubsection{Duration}

Durations of the syllables are analyzed with respect to positional and contextual factors. For detailed duration analysis, syllables are categorized into groups based on size of the word and position of the word in the utterance, and the analysis is performed separately on each category. From the duration analysis, it is observed that durations of sound units depend on several factors at various levels, and it is very difficult to derive precise rules for accurate estimation of durations. Therefore, there is a need to explore nonlinear models to capture the duration patterns of sound units from features [23].

The factors affecting the duration of the basic sound units can be broadly categorized into phonological, positional and contextual. The vowel is considered as the nucleus of a syllable, and consonants may be present on either side of the vowel. The duration of a syllable is influenced by the position of the vowel, the category of the vowel and the type of the consonants associated with the vowel. The contextual factors include the preceding and the following syllables. In addition, the gender of the speaker, psychological state of the speaker, age etc. also affects the duration. For Example, consider the Punjabi sentence में ठிव Jं (maim thīk hāṃ)। The duration of the word "ठीव" (thîk) can vary depending upon the mood of the speaker.

\subsubsection{F0 Contour}

F0 is usually defined, for voiced speech, as the rate of vibration of the vocal folds. F0 is considered one of the most important features for the characterisation of emotions and is the acoustic correlate of the perceptive pitch. Fundamental frequency, also known as pitch, is usually the lowest frequency component, or partial, which relates well to most of the other partials. In a periodic waveform, most partials are harmonically related, meaning that the frequency of most of the partials is related to the frequency of the lowest partial by a small whole-number ratio. The frequency of this lowest partial is the fundamental frequency of the waveform and how this fundamental frequency changes over the period of time is determined by the F0 contour [23].

Figure 4, shows the example of F0 contour of the word "मलगगाभ" (shalgam) 


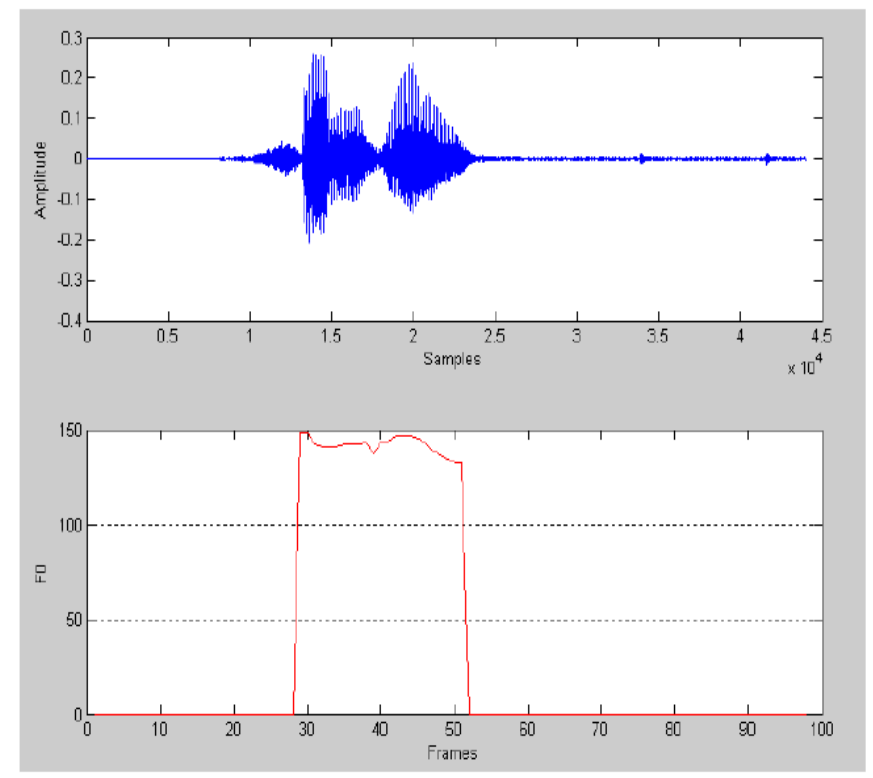

Figure 4. Signal and F0 of "मूलूगम" (shalgam)showing zero value of F0 in unvoiced region

\subsubsection{Energy Contour}

In linguistics, speech synthesis and music, the energy contour of a sound is a function or curve that tracks the perceived pitch of the sound over time. Energy contour may include multiple sounds utilizing many pitches, and can relate to frequency function at one point in time to the frequency function at a later point. It is fundamental to the linguistic concept of tone, where the pitch or change in pitch of a speech unit over time affects the semantic meaning of a sound. It also indicates intonation in pitch accent languages.

One of the primary challenges in speech synthesis technology, is to create a natural-sounding energy contour for the utterance as a whole. Unnatural energy contours result in synthesis that sounds lifeless or emotionless to human listeners, a feature that has become a stereotype of speech synthesis in popular culture.

\section{METHODS OF SPEECH SYNTHESIS}

Synthesized speech can be produced by several different methods. All of these have some benefits and deficiencies. The methods are usually classified into three groups [5]. These are as follows:

- Formant synthesis, which is done by exciting a set of resonators by a voicing source or noise generator to achieve the desired speech spectrum.

- Concatenative synthesis, which uses different length pre-recorded samples derived from natural speech.

- $\quad$ Statistical parametric synthesis, which is based on Hidden Markov Models (HMM). 
Figure 5 shows the various methods for Wave-Form Generation. The formant and concatenative methods are the most commonly used in most synthesis systems. The Statistical parametric synthesis is still too complicated for high quality implementations, but may arise as a potential method in the future [6].

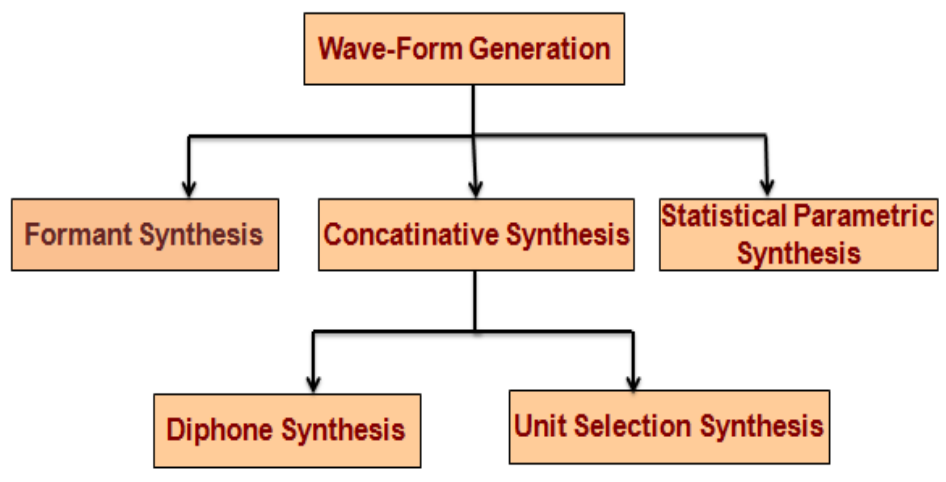

Figure 5. Wave-Form Generation Methods

\subsection{Formant Synthesis}

The most widely used synthesis method during last decades has been formant synthesis which is based on the source-filter-model of speech. It consists of artificial reconstruction of the formant characteristics to be produced. This is done by exciting a set of resonators by a voicing source or noise generator to achieve the desired speech spectrum. Parameters such as fundamental frequency, voicing and noise levels are varied over time to create a waveform of artificial speech [7]. This method is sometimes called rules-based synthesis. Figure 6 shows the typical architecture of formant synthesis.

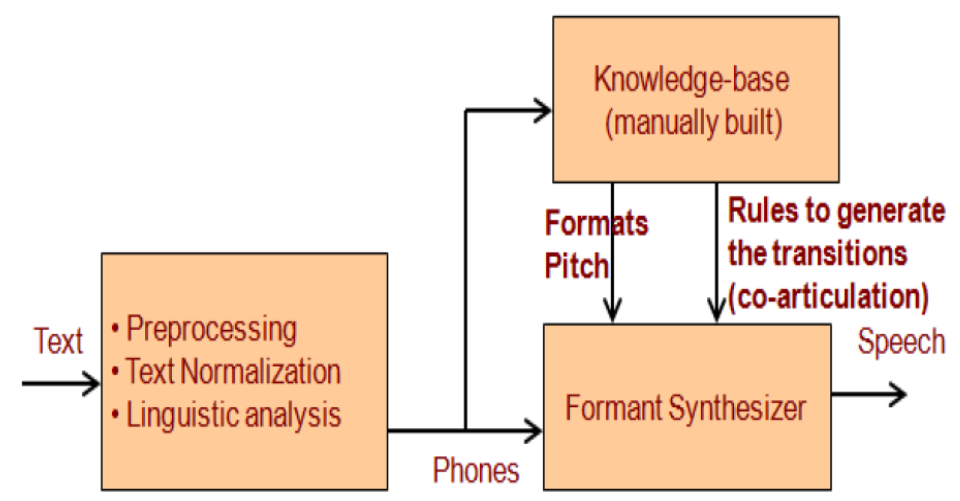

Figure 6. Architecture of Formant Synthesis

\subsection{Concatenative Synthesis}

Concatenative synthesis is based on the concatenation of segments of recorded speech. In this method, waveform segments are stored in a database. For a given text, these segments are joined based on some joining rules. Connecting pre-recorded natural utterances is probably the easiest 
way to produce intelligible and natural sounding synthetic speech. One of the most important aspects in concatenative synthesis is to find correct unit length. The selection is usually a tradeoff between longer and shorter units. With longer units, high naturalness, less concatenation points and good control of coarticulation are achieved, but the amount of required units and memory is increased. With shorter units, less memory is needed, but the sample collecting and labelling procedures become more difficult and complex [8].

For example, for Punjabi language, if longer units contains triphones, i.e., CVC pairs, then memory will increase as there would be the combination of all the consonants firstly with all the vowels and then those combinations again with all the consonants, so memory requirement for saving those units will increase as compared to the shorter units wherein when single consonants and vowels are saved as individual units, it will take a very less memory. There are two main sub-types of concatenative synthesis [9][10], as given below:

- Unit selection synthesis

- Diphone synthesis

Figure 7 shows the architecture of concatenation based synthesis.

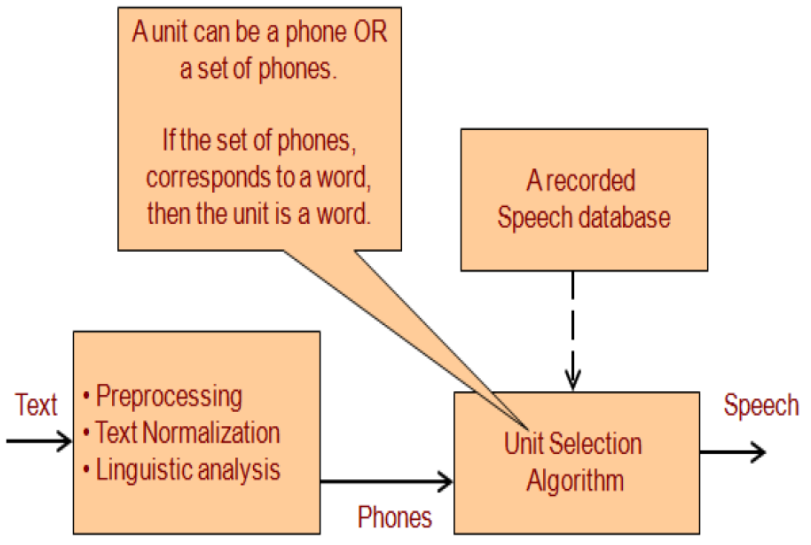

Figure 7. Architecture of concatenation based synthesis

In unit selection synthesis large databases of recorded speech are used [9]. The primary motivation for the use of large databases is that with a large number of units available with varied prosodic and spectral characteristics it should be possible to synthesize more natural-sounding speech than that can be produced with a small set of controlled units [10]. During database creation, each recorded utterance is segmented into individual phones, diphones, half-phones, syllables, morphemes, words, phrases, and sentences [11]. Figure 8, shows the architecture of unit selection process. 


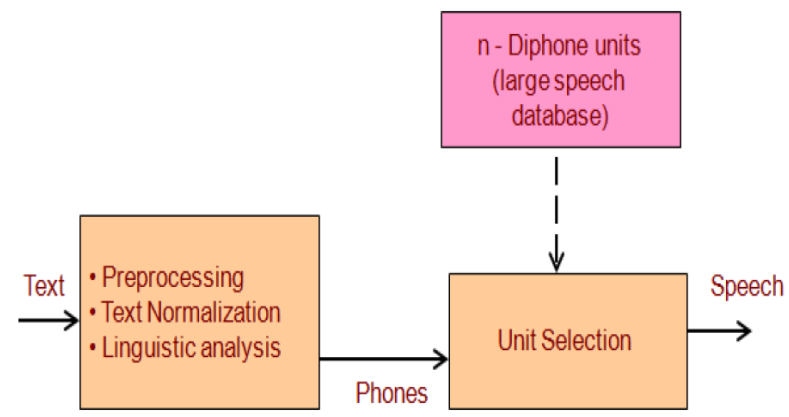

Figure 8. Architecture of unit selection process

Diphone synthesis uses a minimal speech database containing all the diphones occurring in a language. In diphone synthesis, only one example of each diphone is contained in the speech database. The quality of resulting speech is generally worse than that of unit-selection systems, but more natural-sounding than the output of formant synthesizers [7]. Figure 9 shows the snapshot of diphone.

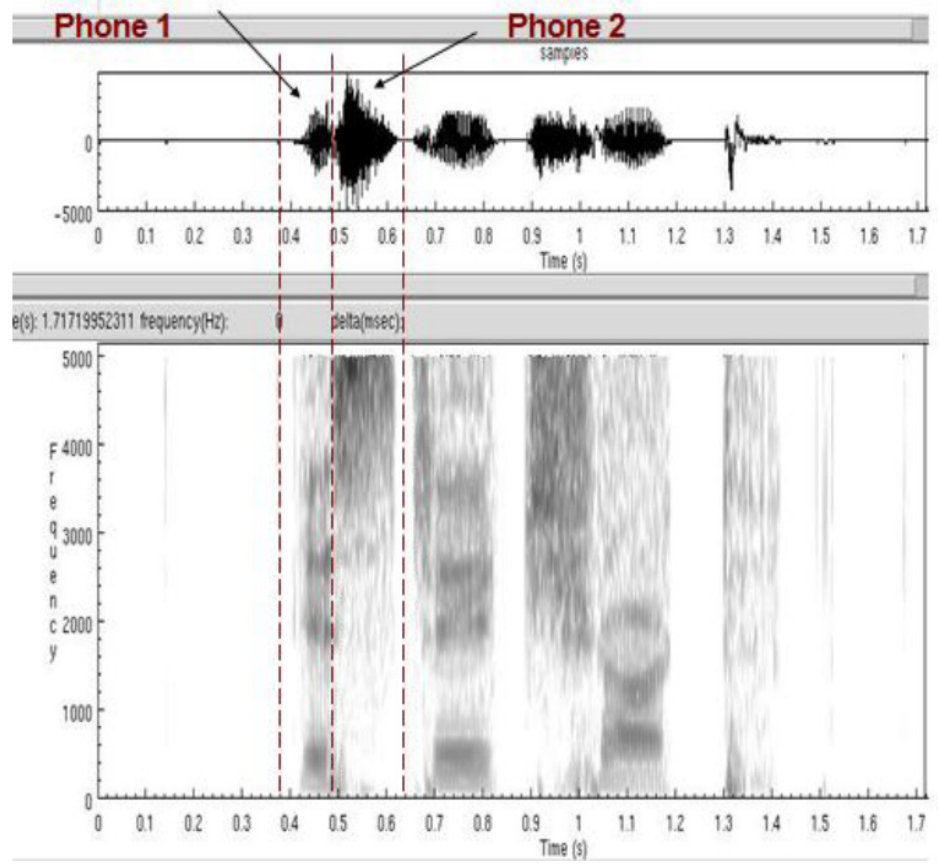

Figure 9. Snapshot of diphone

\subsection{Statistical Parametric Synthesis}

Statistical Parametric Synthesis (SPS) is one of the latest trends in TTS system. The SPS methods produce speech from a set of parameters learned from the speech data. Unlike traditional parametric synthesis methods which require manual specification and hand-tuning of the parameters, the SPS methods use statistical machine learning models such as Classification and Regression Tree (CART), HMMs, etc. to estimate the parameters of speech sounds and their 
dynamics. The SPS methods offer simplicity in storage by encoding the speech data in terms of a compact set of parameters, and also provide mechanisms for manipulation of prosody, voice conversion etc. The SPS methods are found to produce intelligible and consistent speech as compared to natural and often inconsistent speech by unit selection techniques [12].

\section{TEXT-TO-SPEECH SYSTEMS FOR INDIAN LANGUAGES}

In order to help visually impaired and vocally disabled persons and to cater to the needs of day to day increasing applications of TTS system, development of innovative TTS systems has become a necessity in India. Different TTS systems have been built for Indian languages. Some of them are described below.

\subsection{Dhvani- Indian Language Text-To-Speech System}

Dhvani is a Text-To-Speech System specially designed for Indian languages. It has been developed by Simputer trust headed by Dr. Ramesh Hariharan at Indian Institute of Science Bangalore in year 2000. This system is available online at http://dhvani.sourceforge.net/. It uses diphone concatenation algorithm. Currently this system has Hindi, Malayalam, Kannada, Bengali, Oriya, Punjabi, Gujarati, Telugu and Marathi modules. All sound files stored in the database are compressed files. It has different modules for every language. It is based on observation that a direct grapheme to phoneme mapping exits for all Indian languages in general. It is an attempt in India to cover all Indian languages under a single framework. In this system, each language requires a Unicode parser [14] [15].

\subsection{Shruti: An Embedded Text-To-Speech System for Indian Languages}

Mukhopadhyay et al. have developed Shruti system in year 2006 at Indian Institute of Technology Kharagpur. It is a Text-To-Speech system, which has been developed using a concatenative speech synthesis technique. This is the Text-To-Speech system built specifically for two of the Indian languages, namely Bengali and Hindi [16][13].

\subsection{HP Labs India TTS System}

A Hindi TTS system was developed at HP Labs India on a generic TTS framework that it created based on Festival [24]. Festival is available online at http://www.cstr.ed.ac.uk/projects/festival/. This involved extending the Natural Language Processing module of Festival by providing tools that were more appropriate for handling non-European languages like Hindi. It also incorporated methodology and tools for creating TTS speech databases, from best practices for choosing a voice talent to tools for automatic segmentation and annotation of the database [17][18]. The TTS framework is illustrated in Figure 10 [25]. 


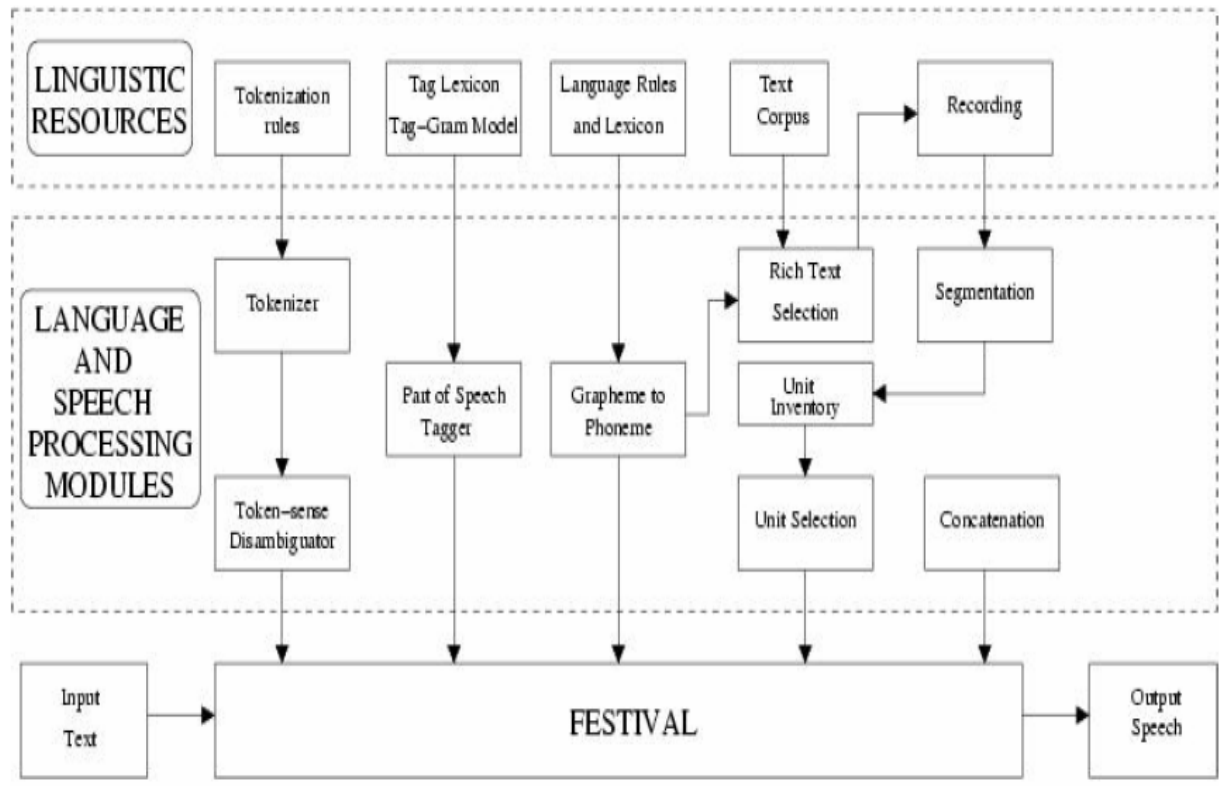

Figure 10. TTS framework created by HP Labs, India

\subsection{Vani: An Indian Language Text-To-Speech Synthesizer}

Vani is an Indian Language Text-To-Speech synthesizer developed at IIT Bombay, India. This system is available online at http://www.cse.iitb.ac.in/vani/. Generally, all existing TTS systems allow user to specify what is to be spoken but does not give any control on how it has to be spoken. In Vani, a new encoding scheme has been introduced called vTrans. A vTrans file makes a person to encode what text he wants to be spoken and also the way that text to be spoken. A signal processing module is then used to bring out this speech by making appropriate variations to the sound database.

vTrans is an XML document that contains a head and a body. In the head part of XML document the parameters and styles are defined. These parameters are pitch, volume and duration. The body section contains several tags. They may be nested, but text is put only in the innermost of the tags. The text within the tags is ITrans encoded. The tags may be any of the parameters defined in the head section. The attributes assigned to these tags determines which style to use and allow the user to scale and translate the function to be used as required [19] [20].

\section{COMPARISON OF INDIAN LANGUAGES TTS SYSTEMS}

In the above sections, four TTS systems have been discussed, namely Dhvani, Shruti, HP Lab system and Vani system. These systems are compared in Table I on the basis of languages it support, size of the speech units, methodologies used to code, store and synthesize the speech and prosody [26]. 


\section{CONCLUSION}

This paper describes the process of development of a TTS system for Punjabi Language. A general Architecture and various components, namely, Text Pre-processing, Text Normalization, Linguistic Analysis, Prosody Information and Waveform Generation of this system have been discussed.

Three Waveform generation approaches, namely, Formant based approach, Concatinative based approach and statistical parametric based synthesis have also been presented in this work. An illustration on various existing TTS systems for Indian Languages has also been included in this paper and these systems have been compared on a few parameters. The further scope of this work shall include the development of various components of a TTS system for Punjabi Language and integrating them in a fully functional TTS system.

Table 1. Comparison of Indian Languages TTS systems

\begin{tabular}{|c|c|c|c|c|c|c|c|}
\hline $\begin{array}{l}\text { S. } \\
\text { No. }\end{array}$ & $\begin{array}{c}\text { Name of the } \\
\text { system }\end{array}$ & $\begin{array}{l}\text { Language } \\
\text { supported }\end{array}$ & $\begin{array}{l}\text { Synthesis } \\
\text { Technique }\end{array}$ & Database/Unit & $\begin{array}{c}\text { Text } \\
\text { processing/ } \\
\text { Tools }\end{array}$ & Prsosody & Phonetics \\
\hline 1 & $\begin{array}{l}\text { Dhvani- } \\
\text { Indian } \\
\text { Language } \\
\text { Text-To- } \\
\text { Speech } \\
\text { system }\end{array}$ & $\begin{array}{c}\text { Hindi, } \\
\text { Malayalam, } \\
\text { Kannada, } \\
\text { Bengali, } \\
\text { Oriya, } \\
\text { Punjabi, } \\
\text { Gujrati, } \\
\text { Telegu. }\end{array}$ & $\begin{array}{c}\text { Diphone } \\
\text { Concatination }\end{array}$ & $\begin{array}{l}\text { Syllable } \\
\text { Database }\end{array}$ & $\begin{array}{l}\text { Parsing rule } \\
\text { for } \\
\text { Phonetization }\end{array}$ & $\begin{array}{c}\text { Not } \\
\text { supported }\end{array}$ & Not Used \\
\hline 2 & $\begin{array}{l}\text { Shruti: an } \\
\text { embedded } \\
\text { Text-To- } \\
\text { Speech } \\
\text { system for } \\
\text { Indian } \\
\text { Languages }\end{array}$ & $\begin{array}{c}\text { Bengali and } \\
\text { Hindi }\end{array}$ & $\begin{array}{c}\text { Concatination } \\
\text { synthesis }\end{array}$ & $\begin{array}{l}\text { Phoneme } \\
\text { Database }\end{array}$ & $\begin{array}{l}\text { Parsing rules } \\
\text { for } \\
\text { Phonetization }\end{array}$ & $\begin{array}{l}\text { Prosodic and } \\
\text { intonational } \\
\text { rules are } \\
\text { applied }\end{array}$ & Used \\
\hline 3 & $\begin{array}{l}\text { TTS System } \\
\text { by HP Labs, } \\
\text { India. }\end{array}$ & Hindi & $\begin{array}{c}\text { Concatination } \\
\text { synthesis }\end{array}$ & $\begin{array}{c}\text { Phoneme or } \\
\text { syllable } \\
\text { Database }\end{array}$ & $\begin{array}{c}\text { Festival } \\
\text { Based- } \\
\text { FestVox } \\
\text { tools }\end{array}$ & $\begin{array}{c}\text { Not } \\
\text { supported }\end{array}$ & Not Used \\
\hline 4 & $\begin{array}{c}\text { Vani: an } \\
\text { Indian } \\
\text { Language } \\
\text { Text-To- } \\
\text { Speech } \\
\text { synthesizer }\end{array}$ & Hindi & $\begin{array}{c}\text { Concatination } \\
\text { Sunthesis }\end{array}$ & $\begin{array}{c}\text { Fract } \\
\text { Phoneme } \\
\text { Database }\end{array}$ & $\begin{array}{l}\text { Encoding } \\
\text { Scheme } \\
\text { called } \\
\text { vTrams }\end{array}$ & $\begin{array}{l}\text { Parameters to } \\
\text { control } \\
\text { speech like } \\
\text { pitch, volume } \\
\text { and duration } \\
\text { is given by } \\
\text { user }\end{array}$ & Not Used \\
\hline
\end{tabular}

\section{REFERENCES}

[1] D. Klatt, "Review of Text-To-Speech Conversion for English", Journal of the Acoustical Society of America, JASA, no. 3, vol. 82, 1987, pp. 737-793. 
[2] S.P. Kishore, R. Kumar, R. Sangal, “A data-driven synthesis approach for Indian languages using syllable as basic unit", in Proc. International. Conference on Natural Language Processing (ICON), 2002, pp. 311-316.

[3] M.H. O`Malley, “Text-To-Speech conversion Technology”, IEEE Computer, vol. 23, 1990, pp. 1723.

[4] R. Sproat, A. W. Black, S. Chen, S. Kumar, M. Ostendorf, and C. Richards., "Normalization of nonstandard words", Computer Speech and Language, no. 3, vol. 15, 2001, pp. 287-333.

[5] S. Lemmetty, "Review of Speech Synthesis Technology", MS Thesis, Electrical and Communications Engineering, Helsinki University of Technology, 1999.

[6] S. Thomas, "Natural sounding Text-To-Speech synthesis based on Syllable-like units", MS thesis, Department of Computer Science and Engineering, Indian Institute of Technology, Madras, 2007.

[7] A. Chauhan, V. Chauhan, S.P. Singh, A.K. Tomar, H. Chauhan, "A Text-To-Speech System for Hindi using English Language”, International Journal of Computer Science and Technology, no. 3, vol. 2, 2011, pp. 322-326.

[8] P. Chaudhury, M. Rao, K.V. Kumar, "Symbol based concatenation approach for Text-To-Speech System for Hindi using vowel classification technique", in Proc. World Congress on Nature and biologically Inspired computing, 2002, pp. 1082 - 1087.

[9] S.P. Kishore, A.W. Black, "Unit Size in Unit Selection Speech Synthesis", in Proc. EUROSPEECH 2003, Geneva, Italy, 2003.

[10] A.J. Hunt, A.W. Black, "Unit selection in a concatenative speech synthesis system using a large speech database", in Proc. IEEE international Conference on Acoustics, Speech and Signal Processing, Atlanta, GA, USA, vol. 1, 2011, pp. 373 - 376.

[11] N.S. Krishna, H.A. Murthy, T.A. Gonsalves, "Text-To-Speech in Indian Languages", in Proc. International Conference on Natural Language Processing, ICON-2002, Mumbai, 2002, pp. 317-326.

[12] A. W. Black and K. Tokuda, "The Blizzard Challenge - 2005: Evaluating corpus based speech synthesis on common datasets", in Proc. INTERSPEECH, Lisbon, Portugal, 2005, pp. 77-80.

[13] A. Basu, D. Sen, S. Sen and S. Chakraborty, "An Indian Language Speech Synthesizer - Techniques and Applications", in Proc. National Systems Conference, NSC 2003, Indian Institute of Technology, Kharagpur, India, 2003, pp. 217-223.

[14] S. Thottingal, (december 2012) "Dhvani Indian Language Text-To-Speech System", http://foss.in/2007/register/slides/Dhvani/, 2007.

[15] R. Hariharan, (April 2013) [Online], http://dhvani.sourceforge.net/, 2007.

[16] A. Mukhopadhyay, S. Chakraborty, M. Choudhury, A. Lahiri, S. Dey, A. Basu, "Shruti- an Embedded Text-To-Speech System for Indian Languages", in Proc. IEEE Proceedings on Software Engineering, no. 2, vol. 153,2006 , pp. 75-79.

[17] A.G. Ramakrishnan, K. Bali, “Tools for the Development of a Hindi Speech Synthesis System”, in Proc. 5th ISCA Speech Synthesis Workshop, Pittsburgh, 2004, pp. 109-114.

[18] A.K. Singh, "A Computational Phonetic Model for Indian Language Scripts", in Proc. Fifth International Workshop on Writing Systems, Nijmegen, Netherlands, 2006. 
[19] H. Jain(April 2013) [Online]. http://www.cse.iitb.ac.in/vani/, 2004.

[20] H. Jain, V. Kanade, K. Desikan, "Vani-An Indian Language Text-To-Speech synthesizer", Project Report, Department of Computer Science and Engineering, IIT, Bombay, 2004.

[21] A. W. Black and P. Taylor, "Assigning Intonation Elements and Prosodic Phrasing for English Speech Synthesis from high level Linguistic input", in Proc. of ICSLP, Yokohama, Japan, 1994, pp. 715-718.

[22] P. Ladefoged and K. Johnson, A course in Phonetics, Cengage learning, 2010.

[23] P. Sharma, "Automatic Identification of Silence, Voiced and Unvoiced Chunks in Speech", ME Thesis, SMCA, Thapar University, 2012.

[24] Alan black and Rob Clark, (April 2013) [Online] http://www.cstr.ed.ac.uk/projects/festival/, 2004.

[25] Kalika Bali, N. Sridhar Krishna, Sameer Badasker, KSR Anjaneyulu, "Enabling IT Usage through the Creation of a High Quality Hindi Text-to-Speech System", Project Report, HP Laboratories, India, 2007.

[26] S. Gupta, "Hindi Text To Speech System", ME Thesis, Computer Science and Engineering Department, Thapar University, Patiala, 2012.

[27] P. Singh and G.S. Lehal, "Corpus Based Statistical Analysis of Punjabi Syllables for Preparation of Punjabi Speech Database", International Journal of Intelligent Computing Research (IJICR), No. 3, Volume 1, June 2010.

[28] L.L. Cherry and W. Vesterman., "Writing tools - the STYLE and DICTION programs", 4.3 BSD UNIX System Documentation, University of California, Berkeley, 1991.

[29] A. Mikheev., "Periods, capitalized words, etc." Computational Linguistics, No. 3, Volume 28, 2002, pp. 289-318.

[30] A. Voutilainen., "A syntax-based part of speech analyser." in Proc. of the Seventh Conference of the European Chapter of the Association for Computation al Linguistics, Dublin. Association for Computational Linguistics, 1995, pp. 157.164.

[31] F. Jelinek., "Markov source modeling of text generation." The Impact of Processing Techniques on Communications, NATO ASI series, 1985, pp. 569.598.

[32] E. Brill., "Transformation-based error-driven learning and natural language processing: A case study in part of speech tagging." Computational Linguistics, No. 4, Volume 21, 1995, pp. 543-566.

\section{AUTHORS}

Rupinderdeep Kaur is Lecturer in Computer Science and Engineering Department at Thapar University, Patiala. She has more than 6 years of academic experience. She has done her B. Tech from Chandigarh Engineering College and M.E. in Software Engineering from Thapar University. She is Persuing her Ph.D. in "Prosody based Text-To-Speech System for Punjabi Language" from Thapar University.

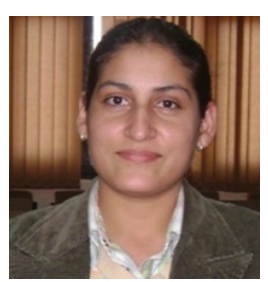


R.K. Sharma is professor in the Computer Science and Engineering Dapartment at Thapar University, Patiala. He has more than 23 years of teaching experience. He has earned his Ph.D. degree in 1993 from IIT Roorkie on "Computer-Aided Simulation Studies Exploring Efficient Mixing-Type Estimators". He has published more than 90 research papers in Journals, Conferences and Magazines of repute. He has Guided 15 students for $\mathrm{PhD}$ work.

Parteek Kumar is Associate Professor in the Computer Science and Engineering Dapartment at Thapar University, Patiala. He has more than 18 years of academic experience. He has earned his B.Tech from SLIET, MS from BITS Pilani, Ph.D from Thapar University. He has published more than 50 research papers and articles in Journals, Conferences and Magazines of repute.
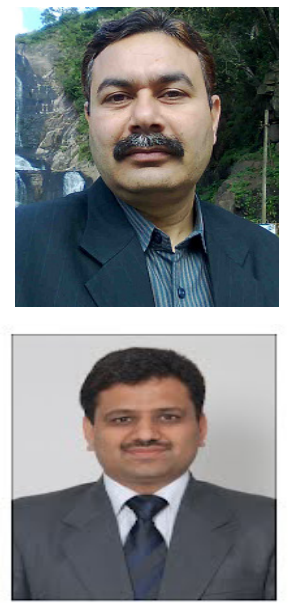


\title{
KURTOSIS: IS IT AN APPROPRIATE MEASURE TO COMPARE THE EXTENT OF FAT-TAILEDNESS OF THE DEGREE DISTRIBUTION FOR ANY TWO REAL- WORLD NETWORKS?
}

\author{
Natarajan Meghanathan \\ Jackson State University, MS, USA
}

\begin{abstract}
"Kurtosis" has long been considered an appropriate measure to quantify the extent of fattailedness of the degree distribution of a complex real-world network. However, the Kurtosis values for more than one real-world network have not been studied in conjunction with other statistical measures that also capture the extent of variation in node degree. In this paper, we determine the Kurtosis values for a suite of 48 real-world networks along with measures such as $\operatorname{SPR}(K), \operatorname{Max}(K)-\operatorname{Min}(K), \operatorname{Max}(K)-\operatorname{Avg}(K), S D(K) / \operatorname{Avg}(K)$, wherein $\operatorname{SPR}(K), \operatorname{Max}(K), \operatorname{Min}(K)$, $A v g(K)$ and $S D(K)$ represent the spectral radius ratio for node degree, maximum node degree, minimum node degree, average and standard deviation of node degree respectively. Contrary to the conceived notion in the literature, we observe that real-world networks whose degree distribution is Poisson in nature (characterized by lower values of $\operatorname{SPR}(K), \operatorname{Max}(K)-\operatorname{Min}(K)$, $\operatorname{Max}(K)-\operatorname{Avg}(K), S D(K) / A v g(K))$ could have Kurtosis values that are larger than that of realworld networks whose degree distribution is scale-free in nature (characterized by larger values of $\operatorname{SPR}(K), \operatorname{Max}(K)-\operatorname{Min}(K), \operatorname{Max}(K)-A v g(K), S D(K) / A v g(K))$. When evaluated for any two realworld networks among all the 48 real-world networks, the Kendall's concordance-based correlation coefficients between Kurtosis and each of $\operatorname{SPR}, \operatorname{Max}(K)-\operatorname{Min}(K), \operatorname{Max}(K)-\operatorname{Avg}(K)$ and $S D(K) / A v g(K)$ are $0.40,0.26,0.34$ and 0.50 respectively. Thus, we seriously question the appropriateness of using Kurtosis to compare the extent of fat-tailedness of the degree distribution of the vertices for any two real-world networks.
\end{abstract}

\section{KEYWORDS}

Fat-tailedness, Degree Distribution, Kurtosis, Real-World Networks, Kendall's Concordancebased Correlation Coefficient

\section{INTRODUCTION}

Complex network analysis is about analyzing complex real-world networks from a graph theoretic perspective [1]. Several measures from Statistics are also used to infer the distribution of the node-level metrics [2]. One such metric and distribution that are of interest in this paper is the degree centrality metric and the fat-tailedness of its distribution. The degree of a vertex is the number of neighbors for the vertex. A degree distribution is considered to be fat-tailed if the maximum degree for a vertex is much different from the minimum or the average degree for the vertex (correspondingly, the standard deviation of node degree is also comparable or even larger than that of the average node degree) [3]. Poisson degree distributions (characteristic of random networks [4]) are not fat-tailed; whereas, power-law degree distributions (characteristic of scalefree networks [5]) are fat-tailed. Real-world networks typically exhibit power-law degree

Natarajan Meghanathan et al. (Eds) : ACSIT, SIPM, FCST, CoNeCo, CMIT - 2017

pp. 89-99, 2017. (C) CS \& IT-CSCP 2017

DOI : $10.5121 /$ csit.2017.70807 
distribution [3]; however the extent of fat-tailedness of the distribution differs among the networks.

Until now, the Kurtosis measure has been perceived to be the most appropriate measure that could be used to quantify the extent of fat-tailedness of the degree distribution of the vertices in a real-world network [2]. But, there is no formal work that determined the Kurtosis of a suite of real-world networks of diverse degree distributions and analyzed whether the Kurtosis of a network with smaller variation in node degree (i.e., less fat-tailed) is more likely to be larger than the Kurtosis of a network with a relatively larger variation in node degree (i.e., more fat-tailed). This forms the motivation for our research in this paper. We measure the Kurtosis of the degree distributions for a suite of 48 real-world networks in conjunction with several other relevant metrics that also capture the extent of variation in node degree. Let $\operatorname{SPR}(K), \operatorname{Max}(K), \operatorname{Min}(K)$, $\operatorname{Avg}(K)$ and $\operatorname{SD}(K)$ represent the spectral radius ratio for node degree, maximum node degree, minimum node degree, average and standard deviation of node degree respectively. The metrics that are explored in this research along with Kurtosis for node degree are: $\operatorname{SPR}(K), \operatorname{Max}(K)$ $\operatorname{Min}(K), \operatorname{Max}(K)-\operatorname{Avg}(K)$ and $\operatorname{SD}(K) / \operatorname{Avg}(K)$. The spectral radius ratio for node degree $(\operatorname{SPR}(K))$ [6] is defined as the ratio of the principal eigenvalue of the adjacency matrix of the network graph to that of the average node degree. According to literature [7], $\operatorname{Min}(K) \leq \operatorname{Avg}(K) \leq$ Principal $\operatorname{Eigenvalue}(K) \leq \operatorname{Max}(K)$. The smaller the difference between $\operatorname{Max}(K)$ and $\operatorname{Min}(K)$ for a network, the lower the value for $\operatorname{SPR}(K)=$ Principal Eigenvalue $(K) / \operatorname{Avg}(K)$. SPR $(K)$ values start from 1.0 and this is the value expected for a truly random network.

We seek to explore whether or not a real-world network A with larger Kurtosis for node degree than a real-world network B also incurs larger values for one of these above metrics that also capture the extent of variation in node degree. We measure the Kendall's concordance-based correlation coefficient [8] for Kurtosis with each of the above four metrics for the suite of 48 realworld networks. We say two networks $A$ and $B$ are concordant with respect to any two metrics (say, $\mathrm{X}$ and $\mathrm{Y}$ ) if $\mathrm{X}(\mathrm{A})<\mathrm{X}(\mathrm{B})$ and $\mathrm{Y}(\mathrm{A})<\mathrm{Y}(\mathrm{B})$ or $\mathrm{X}(\mathrm{A})>\mathrm{X}(\mathrm{B})$ and $\mathrm{Y}(\mathrm{A})>\mathrm{Y}(\mathrm{B})$ or $\mathrm{X}(\mathrm{A})=$ $\mathrm{X}(\mathrm{B})$ and $\mathrm{Y}(\mathrm{A})=\mathrm{Y}(\mathrm{B})$. Surprisingly, we observe the Kendall's concordance-based correlation coefficient for Kurtosis with each of $\operatorname{SPR}(K), \quad \operatorname{Max}(K)-\operatorname{Min}(K), \quad \operatorname{Max}(K)-\operatorname{Avg}(K)$ and $\mathrm{SD}(K) / \operatorname{Avg}(K)$ to be low: $0.40,0.26,0.34$ and 0.50 respectively; thus, seriously raising the question of using Kurtosis to compare the extent of fat-tailedness of the degree distribution of real-world networks when it has lower correlation with metrics that also capture the extent of variation in node degree.

The rest of the paper is organized as follows: Section 2 illustrates the computation of Kurtosis for a real-world network with an example graph. Section 3 illustrates the computation of the Kendall's concordance-based correlation coefficient (Kurtosis vs. Spectral radius ratio for node degree) for a subset of 8 real-world networks from the suite of 48 real-world networks studied in this research. Section 4 first provides a brief overview of the 48 real-world networks and then presents the values for Kurtosis and the other metrics (stated above) that capture the extent of variation in node degree. Section 4 also discusses the correlation for Kurtosis with each of these metrics. Section 5 concludes the paper. Throughout the paper, the terms 'node' and 'vertex', 'link' and 'edge', 'network' and 'graph' are used interchangeably. They mean the same.

\section{KURTOSIS: FORMULATION AND ILLUSTRATION}

Kurtosis has been traditionally used to quantify the extent of fat-tailedness of the distribution of a random variable. In the context of complex network analysis, Kurtosis has been used to quantify the extent of fat-tailedness of the degree distribution of the vertices in a real-world network. However, there is no formal work that has evaluated its appropriateness for comparing two realworld networks on the basis of the fat-tailedness of the degree distribution of the vertices in 
conjunction with other statistical and spectral metrics that also capture the extent of variation in node degree. In this section, we first present the formulation to compute the Kurtosis of the degree distribution of the vertices and then illustrate the computation with an example graph.

Let $K$ be the set of all degree values for the vertices in a graph. Let $\mathrm{P}(k)$ indicate the probability of finding a vertex with degree $k$, where $k \in K$. The average, standard deviation and kurtosis for node degree are computed as follows:

$$
\begin{array}{r}
\operatorname{Avg}(K)=\sum_{k \in K} P(k) *(k) . \\
\operatorname{SD}(K)=\sqrt{\sum_{k \in K} P(k) *(k-A v g(K))^{2}} \\
\operatorname{Kurtosis}(K)=\frac{\sum_{k \in K} P(k)^{*}(k-\operatorname{Avg}(K))^{4}}{S D(K)^{4}}
\end{array}
$$

\begin{tabular}{|c|c|c|}
\hline ID & Degree & Degree \#Nodes $P(K)$ \\
\hline 0 & 7 & $1 / 10=0.1$ \\
\hline 1 & 5 & $3 / 10=0.3$ \\
\hline 2 & 1 & $3 / 10=0.3$ \\
\hline 3 & 3 & $1 / 10=0.1$ \\
\hline 4 & 4 & $1 / 10=0.1$ \\
\hline 5 & 3 & $1 / 10=0.1$ \\
\hline 6 & 2 & \multirow{5}{*}{$\begin{array}{l}\text { Avg. } \sum_{k} k^{*} P(k) \\
\text { Degree }=\sum_{k} \\
\begin{array}{l}\text { Avg. Degree, Avg(K) } \\
=(1)(0.1)+(2)(0.3)+(3)(0.3) \\
+(4)(0.1)+(5)(0.1)+(7)(0.1) \\
=3.2\end{array}\end{array}$} \\
\hline 7 & 3 & \\
\hline 8 & 2 & \\
\hline 9 & 2 & \\
\hline & & \\
\hline
\end{tabular}

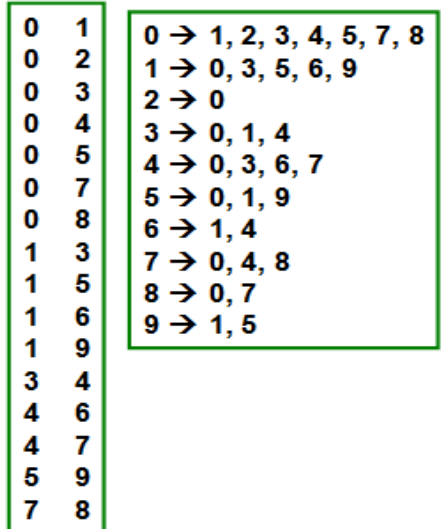

Edge List

\begin{tabular}{|lccccc|}
\hline Degree (k) & $P(k)$ & $(k-A v g(K))^{2}$ & $(k-A v g(K))^{4}$ & $P(k)^{\star}(k-A v g(K))^{2}$ & $P(k)^{\star}(k-A v g(K))^{4}$ \\
1 & 0.1 & 4.84 & 23.43 & 0.484 & 2.343 \\
2 & 0.3 & 1.44 & 2.07 & 0.432 & 0.621 \\
3 & 0.3 & 0.04 & 0.0016 & 0.012 & 0.00048 \\
4 & 0.1 & 0.64 & 0.4096 & 0.064 & 0.04096 \\
5 & 0.1 & 3.24 & 10.498 & 0.324 & 1.0498 \\
7 & 0.1 & 14.44 & 208.51 & 1.444 & 20.851 \\
\hline
\end{tabular}

$$
\begin{gathered}
\operatorname{SD}(\mathrm{K})=\sqrt{\sum_{k} P(k)^{*}(k-A v g(K))^{2}}=\sqrt{2.76}=\underline{\mathbf{1 . 6 6}} \\
\operatorname{Kurtosis}(\mathrm{K})=\frac{\sum_{k} P(k) *(k-A v g(K))^{4}}{S D^{4}}=\frac{. \mathbf{2 4 . 9 1}}{(\mathbf{1 . 6 6})^{4}}=\underline{\mathbf{3 . 2 7}}
\end{gathered}
$$

Figure 1. Example Illustration to Compute the Average, Standard Deviation and Kurtosis of the Degree Distribution of the Vertices in a Graph 
Figure 1 presents an illustration of the computation of the $\operatorname{Avg}(K), \operatorname{SD}(K)$ and $\operatorname{Kurtosis}(K)$ for node degree for an example undirected graph of 10 vertices (whose list of edges is given). We first compute the node degree (the number of neighbors for a vertex) and determine the number of vertices that are of a particular degree. The probability of finding a vertex with a certain degree is simply the fraction of the total number of vertices with the particular degree. Once we have the $k$ vs. $\mathrm{P}(k)$ values for a graph, we can compute the above three statistical metrics using formulations (1), (2) and (3).

\section{KENDALL'S CONCORDANCE-BASED CORRELATION}

The Kendall's concordance-based correlation measure could be used to evaluate the relative ranking of two networks with respect to any two network-level metrics; in our case, Kurtosis vs. any statistical or spectral metric. In this section, we illustrate the computation of the Kendall's concordance-based correlation coefficient for a set of 8 real-world networks (taken from the suite of 48 real-world networks analyzed in Section 4) with respect to Kurtosis and Spectral radius ratio for node degree. Figure 2 illustrates the calculations. We count the number of concordat pairs of networks and the number of discordant pairs of networks and calculate the Kendall's correlation coefficient as the ratio of the sum of the number of concordant pairs and discordant pairs to that of the difference of the number of concordant pairs and discordant pairs.

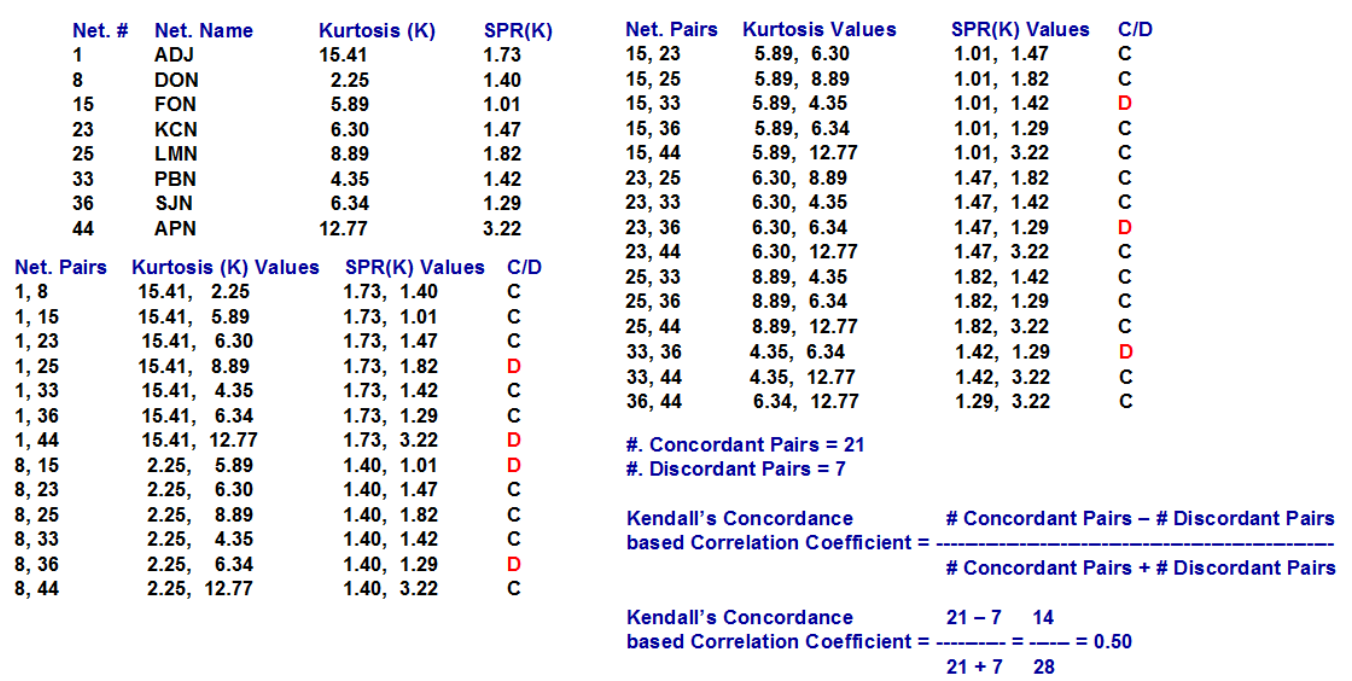

Figure 2. Example Illustration to Compute the Kendall's Concordance-based Correlation Coefficient between Kurtosis and Spectral Radius Ratio for Node Degree for a Subset of the Real-World Networks

A pair of networks $\mathrm{X}$ and $\mathrm{Y}$ are said to be concordant with respect to $\operatorname{Kurtosis}(K)$ and $\operatorname{SPR}(K)$ if either one of the following are true:

(i) $\operatorname{Kurtosis}_{\mathrm{X}}(K)<\operatorname{Kurtosis}_{\mathrm{Y}}(K)$ and $\operatorname{SPR}_{\mathrm{X}}(K)<\operatorname{SPR}_{\mathrm{Y}}(K)$ or

(ii) $\operatorname{Kurtosis}_{\mathrm{X}}(K)>\operatorname{Kurtosis}_{\mathrm{Y}}(K)$ and $\operatorname{SPR}_{\mathrm{X}}(K)>\operatorname{SPR}_{\mathrm{Y}}(K)$ or

(iii) $\operatorname{Kurtosis}_{\mathrm{X}}(K)=\operatorname{Kurtosis}_{\mathrm{Y}}(K)$ and $\operatorname{SPR}_{\mathrm{X}}(K)=\operatorname{SPR}_{\mathrm{Y}}(K)$

A pair of networks $\mathrm{X}$ and $\mathrm{Y}$ are said to be discordant with respect to $\operatorname{Kurtosis}(K)$ and $\operatorname{SPR}(K)$ if either one of the following are true:

(i) $\operatorname{Kurtosis}_{\mathrm{X}}(K)>\operatorname{Kurtosis}_{\mathrm{Y}}(K)$ and $\operatorname{SPR}_{\mathrm{X}}(K) \leq \operatorname{SPR}_{\mathrm{Y}}(K)$ or

(ii) $\operatorname{Kurtosis}_{\mathrm{X}}(K)<\operatorname{Kurtosis}_{\mathrm{Y}}(K)$ and $\operatorname{SPR}_{\mathrm{X}}(K) \leq \operatorname{SPR}_{\mathrm{Y}}(K)$ 
For the set of 8 real-world networks considered in Figure 2 and their $\operatorname{Kurtosis}(K)$ and $\operatorname{SPR}(K)$ values, we observe 21 concordant pairs of networks and 7 discordant pairs of networks; this leads to the Kendall's concordance-based correlation coefficient of $(21-7) /(21+7)=0.50$.

\section{REAL-WORLD NETWORKS AND THEIR CORRELATION ANALYSIS}

In this section, we first introduce the 48 real-world networks analyzed in this paper. Table 1 lists the three character code acronym, name and the network type as well as the number of nodes and edges. The networks considered cover a broad range of categories (as listed below along with the number of networks in each category): Acquaintance network (12), Friendship network (9), Coappearance network (6), Employment network (4), Citation network (3), Literature network (3), Collaboration network (2), Political network (2), Biological network (2), Game network (2), Geographical Network, Transportation network and Trade network (1 each). A brief description about each category of networks is as follows: An acquaintance network is a kind of social network in which the participant nodes slightly (not closely) know each other, as observed typically during an observation period. A friendship network is a kind of social network in which the participant nodes closely know each other and the relationship is not captured over an observation period. A co-appearance network is a network typically extracted from novels/books in such a way that two characters or words (modeled as nodes) are connected if they appear alongside each other. An employment network is a network in which the interaction/relationship between people is primarily due to their employment requirements and not due to any personal liking. A citation network is a network in which two papers (nodes) are connected if one paper cites the other paper as reference. A collaboration network is a network of researchers/authors who are listed as co-authors in at least one publication. A biological network is a network that models the interactions between genes, proteins, animals of a species, etc. A political network is a network of entities (typically politicians) involved in politics. A game network is a network of teams or players playing for different teams and their associations. A literature network is a network of books/papers/terminologies/authors (other than collaboration, citation or coauthorship) involved in a particular area of literature. A transportation network is a network of entities (like airports and their flight connections) involved in public transportation. A trade network is a network of countries/people involved in certain trade. The reader is referred to [9] for a more detailed description of the individual real-world networks.

Table 1. Real-World Networks used in the Correlation Analysis

\begin{tabular}{|c|l|l|l|l|l|l|}
\hline$\#$ & Net. & Net. Description & Ref. & Network Type & \#nodes & \#edges \\
\hline 1 & ADJ & Word Adjacency Network & {$[10]$} & Co-appearance Net. & 112 & 425 \\
\hline 2 & AKN & Anna Karnenina Network & {$[11]$} & Co-appearance Net. & 140 & 494 \\
\hline 3 & JBN & Jazz Band Network & {$[12]$} & Employment Net. & 198 & 2742 \\
\hline 4 & CEN & C. Elegans Neural Network & {$[13]$} & Biological Net. & 297 & 2148 \\
\hline 5 & CLN & Centrality Literature Net. & {$[14]$} & Citation Net. & 118 & 613 \\
\hline 6 & CGD & Citation Graph Drawing Net & {$[15]$} & Citation Net. & 259 & 640 \\
\hline 7 & CFN & Copperfield Network & {$[11]$} & Co-appearance Net. & 89 & 407 \\
\hline 8 & DON & Dolphin Network & {$[16]$} & Acquaintance Net. & 62 & 159 \\
\hline 9 & DRN & Drug Network & {$[17]$} & Acquaintance Net. & 212 & 284 \\
\hline 10 & DLN & Dutch Literature 1976 Net. & {$[18]$} & Literature Net. & 37 & 81 \\
\hline 11 & ERD & Erdos Collaboration Net. & {$[19]$} & Collaboration Net. & 433 & 1314 \\
\hline 12 & FMH & Faux Mesa High School Net & {$[20]$} & Friendship Net. & 147 & 202 \\
\hline 13 & FHT & Friendship in Hi-Tech Firm & {$[21]$} & Friendship Net. & 33 & 91 \\
\hline 14 & FTC & Flying Teams Cade Net. & {$[22]$} & Employment Net. & 48 & 170 \\
\hline 15 & FON & US Football Network & {$[23]$} & Game Net. & 115 & 613 \\
\hline 16 & CDF & College Dorm Fraternity Net & {$[24]$} & Acquaintance Net. & 58 & 967 \\
\hline 17 & GD96 & Graph Drawing 1996 Net & {$[19]$} & Citation Net. & 180 & 228 \\
\hline 18 & MUN & Marvel Universe Network & {$[25]$} & Co-appearance Net. & 167 & 301 \\
\hline
\end{tabular}




\begin{tabular}{|c|c|c|c|c|c|c|}
\hline 19 & GLN & Graph Glossary Network & [19] & Literature Net. & 67 & 118 \\
\hline 20 & HTN & Hypertext 2009 Network & {$[26]$} & Acquaintance Net. & 115 & 2164 \\
\hline 21 & $\mathrm{HCN}$ & Huckleberry Coappear. Net. & [11] & Co-appearance Net. & 76 & 302 \\
\hline 22 & ISP & Infectious Socio-Patterns Net & {$[26]$} & Acquaintance Net. & 309 & 1924 \\
\hline 23 & $\mathrm{KCN}$ & Karate Club Network & [27] & Acquaintance Net. & 34 & 78 \\
\hline 24 & KFP & Korea Family Planning Net. & [28] & Acquaintance Net. & 37 & 85 \\
\hline 25 & LMN & Les Miserables Network & [11] & Co-appearance Net. & 77 & 254 \\
\hline 26 & MDN & Macaque Dominance Net. & {$[29]$} & Biological Net. & 62 & 1167 \\
\hline 27 & MTB & Madrid Train Bombing Net. & {$[30]$} & Acquaintance Net. & 64 & 295 \\
\hline 28 & $\mathrm{MCE}$ & Manufact. Comp. Empl. Net. & {$[31]$} & Employment Net. & 77 & 1549 \\
\hline 29 & MSJ & Soc. Net. Journal Co-authors & {$[32]$} & Co-author Net. & 475 & 625 \\
\hline 30 & AFB & Author Facebook Network & - & Friendship Net. & 171 & 940 \\
\hline 31 & MPN & Mexican Political Elite Net. & {$[33]$} & Political Net. & 35 & 117 \\
\hline 32 & MMN & ModMath Network & [19] & Friendship Net. & 30 & 61 \\
\hline 33 & PBN & US Politics Books Network & [34] & Literature Net. & 105 & 441 \\
\hline 34 & PSN & Primary School Contact Net. & [35] & Acquaintance Net. & 238 & 5539 \\
\hline 35 & PFN & Prison Friendship Network & {$[36]$} & Friendship Net. & 67 & 142 \\
\hline 36 & SJN & San Juan Sur Family Net. & [37] & Acquaintance Net. & 75 & 155 \\
\hline 37 & SDI & Scotland Corp. Interlock Net & [38] & Employment Net. & 230 & 359 \\
\hline 38 & SPR & Senator Press Release Net. & [39] & Political Net. & 92 & 477 \\
\hline 39 & SWC & Soccer World Cup 1998 Net & [19] & Game Net. & 35 & 118 \\
\hline 40 & SSM & Sawmill Strike Comm. Net. & {$[40]$} & Acquaintance Net. & 24 & 38 \\
\hline 41 & TEN & Taro Exchange Network & [41] & Acquaintance Net. & 22 & 39 \\
\hline 42 & TWF & Teenage Female Friend Net. & [42] & Friendship Net. & 47 & 77 \\
\hline 43 & UKF & UK Faculty Friendship Net. & [43] & Friendship Net. & 83 & 578 \\
\hline 44 & APN & US Airports 1997 Network & [19] & Transportation Net. & 332 & 2126 \\
\hline 45 & USS & US States Network & {$[44]$} & Geographical Net. & 49 & 107 \\
\hline 46 & RHF & Residence Hall Friend Net. & [45] & Friendship Net. & 217 & 1839 \\
\hline 47 & WSB & Windsurfers Beach Network & {$[46]$} & Friendship Net. & 43 & 336 \\
\hline 48 & WTN & World Trade Metal Network & [47] & Trade Net. & 80 & 875 \\
\hline
\end{tabular}

Table 2 lists the values for $\operatorname{SPR}(K), \operatorname{Avg}(K), \operatorname{SD}(K), \operatorname{Min}(K), \operatorname{Max}(K)$ and $\operatorname{Kurtosis}(K)$ obtained for these 48 real-world networks. Figure 3 plots the distribution of $\operatorname{Kurtosis}(K)$ vs. each of the following: $\operatorname{SPR}(K), \operatorname{SD}(K) / \operatorname{Avg}(K), \operatorname{Max}(K)-\operatorname{Min}(K)$ and $\operatorname{Max}(K)-\operatorname{Avg}(K)$. We also mention the values for the Kendall's correlation coefficient obtained for $\operatorname{Kurtosis}(K)$ vs. each of these metrics. We observe all the four correlation coefficient values to be less than or equal to 0.50 ; the largest being 0.50 for Kurtosis vs. $\operatorname{SD}(K) / \operatorname{Avg}(K)$ ratio and the lowest being 0.26 for $\operatorname{Kurtosis}(K)$ vs. $\operatorname{Max}(K)-\operatorname{Min}(K)$, an appreciable measure of the extent of variation in node degree and fattailedness nature of the degree distribution.

Table 2. SPR, Avg, SD, Min, Max and Kurtosis Values for the Degree Distribution of the Real-World Networks

\begin{tabular}{|c|l|l|l|l|l|l|l|}
\hline$\#$ & Net. & $\operatorname{SPR}(K)$ & $\operatorname{Avg}(K)$ & $\operatorname{SD}(K)$ & $\operatorname{Min}(K)$ & $\operatorname{Max}(K)$ & $\operatorname{Kurtosis}(K)$ \\
\hline 1 & ADJ & 1.73 & 7.59 & 6.85 & 1 & 49 & 15.41 \\
\hline 2 & AKN & 2.48 & 7.06 & 10.43 & 1 & 71 & 16.97 \\
\hline 3 & JBN & 1.45 & 27.70 & 17.41 & 1 & 100 & 4.54 \\
\hline 4 & CEN & 1.68 & 14.47 & 12.94 & 1 & 134 & 30.18 \\
\hline 5 & CLN & 2.03 & 10.39 & 10.35 & 0 & 66 & 10.30 \\
\hline 6 & CGD & 2.24 & 4.94 & 3.98 & 0 & 20 & 4.27 \\
\hline 7 & CFN & 1.83 & 9.15 & 10.49 & 1 & 82 & 27.46 \\
\hline 8 & DON & 1.40 & 5.13 & 2.93 & 1 & 12 & 2.25 \\
\hline 9 & DRN & 2.76 & 2.68 & 2.06 & 0 & 15 & 10.12 \\
\hline 10 & DLN & 1.49 & 4.38 & 2.96 & 1 & 12 & 2.52 \\
\hline 11 & ERD & 3.00 & 6.07 & 6.69 & 0 & 41 & 10.11 \\
\hline
\end{tabular}


Computer Science \& Information Technology (CS \& IT)

\begin{tabular}{|c|c|c|c|c|c|c|c|}
\hline 12 & FMH & 2.81 & 2.75 & 2.12 & 0 & 13 & 7.29 \\
\hline 13 & FHT & 1.57 & 5.52 & 3.74 & 0 & 16 & 3.41 \\
\hline 14 & FTC & 1.21 & 7.08 & 2.97 & 1 & 16 & 3.82 \\
\hline 15 & FON & 1.01 & 10.66 & 0.88 & 7 & 12 & 5.89 \\
\hline 16 & $\mathrm{CDF}$ & 1.11 & 33.35 & 11.43 & 6 & 52 & 2.87 \\
\hline 17 & GD96 & 2.38 & 2.53 & 3.82 & 1 & 27 & 28.07 \\
\hline 18 & MUN & 2.54 & 3.61 & 3.76 & 1 & 26 & 12.92 \\
\hline 19 & GLN & 2.01 & 3.52 & 3.19 & 0 & 18 & 10.96 \\
\hline 20 & HTN & 1.21 & 37.64 & 18.30 & 1 & 97 & 3.21 \\
\hline 21 & $\mathrm{HCN}$ & 1.66 & 7.95 & 7.34 & 1 & 53 & 19.77 \\
\hline 22 & ISP & 1.69 & 12.45 & 8.33 & 1 & 47 & 4.14 \\
\hline 23 & $\mathrm{KCN}$ & 1.47 & 4.59 & 3.82 & 1 & 17 & 6.30 \\
\hline 24 & KFP & 1.70 & 4.59 & 3.11 & 0 & 13 & 3.99 \\
\hline 25 & LMN & 1.82 & 6.60 & 6.00 & 1 & 36 & 8.89 \\
\hline 26 & MDN & 1.04 & 37.65 & 7.40 & 17 & 55 & 3.24 \\
\hline 27 & MTB & 1.95 & 9.22 & 6.27 & 0 & 29 & 4.91 \\
\hline 28 & MCE & 1.12 & 40.23 & 12.53 & 18 & 76 & 5.64 \\
\hline 29 & MSJ & 3.48 & 2.63 & 2.15 & 1 & 15 & 10.25 \\
\hline 30 & AFB & 2.29 & 10.99 & 8.16 & 0 & 33 & 3.11 \\
\hline 31 & MPN & 1.23 & 6.69 & 3.27 & 2 & 17 & 4.18 \\
\hline 32 & MMN & 1.59 & 4.07 & 2.26 & 0 & 11 & 4.81 \\
\hline 33 & PBN & 1.42 & 8.40 & 5.45 & 2 & 25 & 4.35 \\
\hline 34 & PSN & 1.22 & 46.55 & 19.85 & 8 & 88 & 2.00 \\
\hline 35 & PFN & 1.32 & 4.24 & 2.07 & 1 & 11 & 3.83 \\
\hline 36 & SJN & 1.29 & 4.13 & 2.02 & 1 & 12 & 6.34 \\
\hline 37 & SDI & 1.94 & 3.12 & 2.04 & 0 & 13 & 7.53 \\
\hline 38 & SPR & 1.47 & 10.37 & 7.55 & 1 & 41 & 4.91 \\
\hline 39 & SWC & 1.45 & 6.74 & 4.71 & 1 & 19 & 4.02 \\
\hline 40 & SSM & 1.22 & 3.17 & 1.34 & 1 & 7 & 4.20 \\
\hline 41 & TEN & 1.06 & 3.55 & 0.94 & 3 & 6 & 3.24 \\
\hline 42 & TWF & 1.59 & 3.28 & 1.55 & 0 & 7 & 2.75 \\
\hline 43 & UKF & 1.35 & 13.93 & 8.11 & 2 & 41 & 4.48 \\
\hline 44 & APN & 3.22 & 12.81 & 20.10 & 1 & 139 & 12.77 \\
\hline 45 & USS & 1.24 & 4.37 & 1.72 & 1 & 9 & 2.75 \\
\hline 46 & RHF & 1.27 & 16.95 & 7.76 & 2 & 56 & 6.42 \\
\hline 47 & WSB & 1.22 & 15.63 & 6.53 & 6 & 31 & 2.26 \\
\hline 48 & WTN & 1.38 & 21.88 & 16.33 & 4 & 77 & 5.54 \\
\hline
\end{tabular}

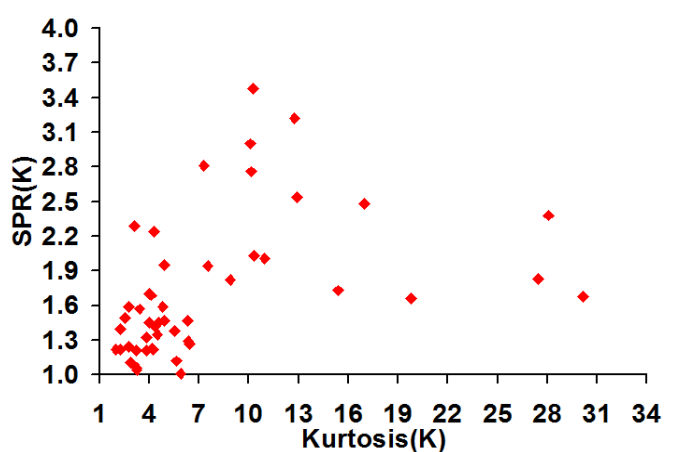

$\operatorname{Kurtosis}(K)$ vs. $\operatorname{SPR}(K)$

Kendall's Correlation Coefficient $=0.40$

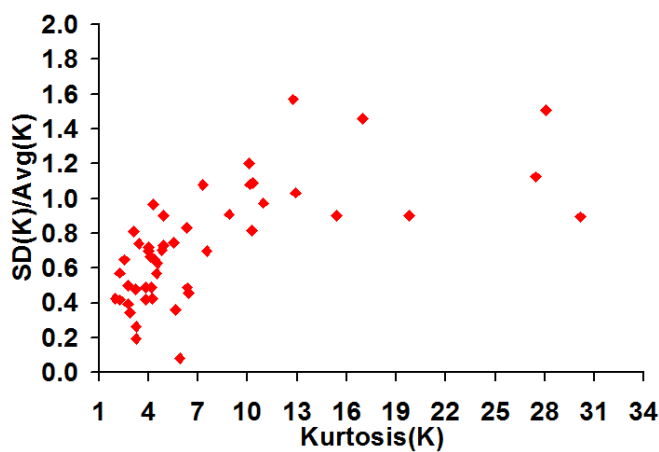

$\operatorname{Kurtosis}(K)$ vs. $\operatorname{SD}(K) / \operatorname{Avg}(K)$

Kendall's Correlation Coefficient $=0.50$ 


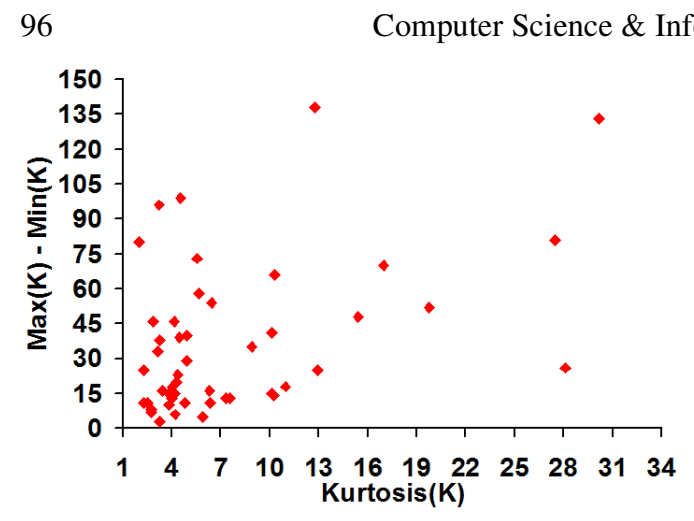

$\operatorname{Kurtosis}(K)$ vs. $\operatorname{Max}(K)-\operatorname{Min}(K)$

Kendall's Correlation Coefficient $=0.26$

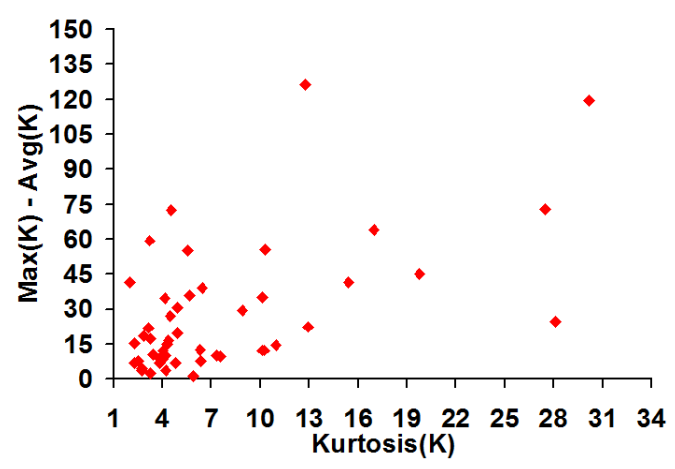

$\operatorname{Kurtosis}(K)$ vs. $\operatorname{Max}(K)-\operatorname{Avg}(K)$ Kendall's Correlation Coefficient $=0.35$

Figure 3. Distribution of the Kurtosis $(K)$ Values vs. $\{\operatorname{SPR}(K), \operatorname{SD}(K) / \operatorname{Avg}(K), \operatorname{Max}(K)-\operatorname{Min}(K)$ and $\operatorname{Max}(K)-\operatorname{Avg}(K)\}$ Values and the Kendall's Correlation Coefficient for the 48 Real-World Networks

\section{RELATED WORK}

In the context of complex network analysis, Kurtosis has been typically used to capture the extent of fat-tailedness of degree distribution of the vertices and make an initial educated guess on the type (i.e., Poisson random networks or Power-law scale-free networks) of degree distribution for an underlying network graph [3]. A real-world network with Kurtosis for the degree distribution greater than 3 is typically considered to be fat-tailed [48]. Kurtosis has also been used to analyze the possibility of existence of outlier(s) in a data set [49]. In the context of complex network analysis, a larger Kurtosis for the degree distribution of a network could imply that the network has one or more nodes with degree(s) that is extremely different from the rest of the nodes in the network [3]. But, the existence of few such outlier nodes is not sufficient to classify a network as a fat-tailed network. We would need the degree distribution to exhibit non-zero probability values for degree values spanning a broader range and exhibit a decreasing trend as the degree values approach the extreme value.

Instead of Kurtosis, several other approaches have also been attempted in the literature to capture the extent of variation in node degree (inclusive of fat-tailedness). For example, graph traversal algorithms like Breadth First Search (BFS) [50] have been used in the literature to analyze the fattailed nature of real-world networks. The BFS algorithm could be used to determine the diameter of a network. The idea proposed in [50is to calculate the diameter $\left(\mathrm{D}_{0}\right)$ of the unperturbed network (with all nodes in the network) and calculate the diameter $\left(D_{i}\right)$ of the network due to the removal of node $i$. The $\Delta_{i}=\mathrm{D}_{i}-\mathrm{D}_{0} / \mathrm{D}_{0}$ value for each node is then calculated. A distribution of probability $\left(\Delta_{i}\right)$ vs. the $\Delta_{i}$ values (for $\Delta_{i}>0$ ) is plotted and if it appears to mimic a power-law distribution, then the network is considered to be fat-free.

\section{CONCLUSIONS}

The high-level contribution of this paper is to illustrate that the Kurtosis measure may not be appropriate to compare any two real-world networks with respect to the extent of fat-tailedness. The Kurtosis of a network with a lower variation in node degree (less fat-tailed) could be larger than the Kurtosis of a network with a relatively larger variation in node degree (relatively more fat-tailed). We measure the Kendall's concordance-based correlation coefficient for Kurtosis with four different statistical/spectral measures that effectively capture the variation in node degree. We observe the correlation coefficients to be no more than 0.50. A possible solution to comprehensively measure and compare the fat-tailedness of degree distributions of a suite of real- 
world networks is to compute the normalized scores of Kurtosis and every other metric (that are also a measure of the extent of variation in node degree) and use a weighted score of all the metrics as a measure of the fat-tailedness of the degree distribution.

\section{REFERENCES}

[1] M. E. J. Newman, Networks: An Introduction. 1st Edition, Oxford University Press, Oxford, UK, May 2010.

[2] E. D. Kolaczyk, Statistical Analysis of Network Data, Springer, Berlin, Germany, March 2009.

[3] A. L. Barabasi, Network Science, 1st Edition, Cambridge University Press, Cambridge, UK.

[4] P. Erdos and A. Renyi, "On Random Graphs I," Publicationes Mathematicae, vol. 6, pp. 290-297, 1959.

[5] A. L. Barabasi and R. Albert, "Emergence of Scaling in Random Networks," Science, vol. 286, no. 5439, pp. 509-512, October 1999.

[6] N. Meghanathan, "Spectral Radius as a Measure of Variation in Node Degree for Complex Network Graphs," Proceedings of the 3rd International Conference on Digital Contents and Applications, pp. 30-33, Hainan, China, December 2014.

[7] B. G. Horne, "Lower Bounds for the Spectral Radius of a Matrix," Linear Algebra and its Applications, September 1997, vol. 263, pp. 261-273.

[8] G. Strang, G. Linear Algebra and its Applications, 4th Edition, Brooks Cole, Pacific Grove, CA, USA, 2006.

[9] N. Meghanathan, "A Computationally-Lightweight and Localized Centrality Metric in lieu of Betweenness Centrality for Complex Network Analysis," Springer Vietnam Journal of Computer Science, vol. 4, no. 1, pp. 23-38, February 2017.

[10] M. E. J. Newman, "Finding Community Structure in Networks using the Eigenvectors of Matrices," Physical Review E, vol. 74, no. 3, 036104, September 2006.

[11] D. E. Knuth, The Stanford GraphBase: A Platform for Combinatorial Computing, 1st Edition, Addison-Wesley, Reading, MA, December 1993.

[12] P. Geiser and L. Danon, "Community Structure in Jazz," Advances in Complex Systems, vo. 6, no. 4, pp. 563-573, July 2003.

[13] J. G. White, E. Southgate, J. N. Thomson and S. Brenner, "The Structure of the Nervous System of the Nematode Caenorhabditis Elegans," Philosophical Transactions B, vol. 314, no. 1165, pp. 1-340, November 1986.

[14] N. P. Hummon, P. Doreian and L. C. Freeman, "Analyzing the Structure of the CentralityProductivity Literature Created between 1948 and 1979," Science Communication, vol. 11, no. 4, pp. 459-480, May 1990. DOI: 10.1177/107554709001100405.

[15] T. Biedl and B. J. Franz, "Graph-Drawing Contest Report," Proceedings of the 9th International Symposium on Graph Drawing, pp. 513-521, September 2001.

[16] D. Lusseau, K. Schneider, O. J. Boisseau, P. Haase, E. Slooten, and S. M. Dawson, "The Bottlenose Dolphin Community of Doubtful Sound Features a Large Proportion of Long-lasting Associations," Behavioral Ecology and Sociobiology, vol. 54, no. 3, pp. 396-405, September 2003. 
[17] J.-S. Lee, "Generating Networks of Illegal Drug Users Using Large Samples of Partial Ego-Network Data," Intelligence and Security Informatics, Lecture Nodes in Computer Science, vol. 3073, pp. 390402, 2004.

[18] W. de Nooy, "A Literary Playground: Literary Criticism and Balance Theory," Poetics, vol. 26, no. 56, pp. 385-404, August 1999.

[19] V. Batagelj and A. Mrvar, Pajek Datasets, http://vlado.fmf.uni-lj.si/pub/networks/data/, 2006.

[20] M. D. Resnick, P. S. Bearman, R. W. Blum, K. E. Bauman, K. M. Harris, J. Jones, J. Tabor, T. Beuhring, R. E. Sieving, M. Shew, M. Ireland, L. H. Bearinger and J. R. Udry, "Protecting Adolescents from Harm. Findings from the National Longitudinal Study on Adolescent Health," Journal of the American Medical Association, vol. 278, no. 10, pp. 823-832, September 1997.

[21] D. Krackhardt, "The Ties that Torture: Simmelian Tie Analysis in Organizations," Research in the Sociology of Organizations, vol. 16, pp. 183-210, 1999.

[22] J. L. Moreno, The Sociometry Reader, The Free Press, pp. 534-547, Glencoe, IL, USA, 1960.

[23] M. Girvan and M. E. J. Newman, "Community Structure in Social and Biological Networks," Proceedings of the National Academy of Sciences of the United States of America, vol. 99, no. 12, pp. 7821-7826, June 2002.

[24] H. R. Bernard, P. D. Killworth and L. Sailer, "Informant Accuracy in Social Network Data IV: A Comparison of Clique-level Structure in Behavioral and Cognitive Network Data," Social Networks, vol. 2, no. 3, pp. 191-218, 1980.

[25] P. M. Gleiser, "How to become a Superhero," Journal of Statistical Mechanics: Theory and Experiments, P09020, September 2007.

[26] L. Isella, J. Stehle, A. Barrat, C. Cattuto, J. F. Pinton and W. Van den Broeck, "What's in a Crowd? Analysis of Face-to-Face Behavioral Networks," Journal of Theoretical Biology, vol. 271, no. 1, pp. 166-180, February 2011. DOI: 10.1016/j.jtbi.2010.11.033.

[27] W. W. Zachary, "An Information Flow Model for Conflict and Fission in Small Groups," Journal of Anthropological Research, vol. 33, no. 4, pp. 452-473, 1977.

[28] E. M. Rogers and D. L. Kincaid, Communication Networks: Toward a New Paradigm for Research, Free Press, June 1980.

[29] Y. Takahata, "Diachronic Changes in the Dominance Relations of Adult Female Japanese Monkeys of the Arashiyama B Group," The Monkeys of Arashiyama, pp. 124-139, Albany: State University of New York Press, 1991.

[30] B. Hayes, "Connecting the Dots," American Scientist, vol. 94, no. 5, pp. 400-404, 2006.

[31] R. L. Cross, A. Parker and R. Cross, The Hidden Power of Social Networks: Understanding How Work Really Gets Done in Organizations, Harvard Business Review Press, 1st Edition, June 2004.

[32] C. McCarty and L. Freeman, http://moreno.ss.uci.edu/data.html, 2008.

[33] J. Gil-Mendieta and S. Schmidt, "The Political Network in Mexico," Social Networks, vol. 18, no. 4, pp. 355-381, October 1996.

[34] V. Krebs, "Proxy Networks: Analyzing One Network to Reveal Another," Bulletin de Méthodologie Sociologique, vol. 79, pp. 61-40, July 2003. 
[35] V. Gemmetto, A. Barrat and C. Cattuto, "Mitigation of Infectious Disease at School: Targeted Class Closure vs. School Closure," BMC Infectious Diseases, vol. 14, no. 695, pp. 1-10, December 2014.

[36] D. MacRae, "Direct Factor Analysis of Sociometric Data," Sociometry, vol. 23, no. 4, pp. 360-371, December 1960.

[37] C. P. Loomis, J. O. Morales, R. A. Clifford and O. E. Leonard, Turrialba Social Systems and the Introduction of Change, pp. 45-78, The Free Press, Glencoe, IL, USA, 1953.

[38] J. P. Scott, The Anatomy of Scottish Capital: Scottish Companies and Scottish Capital, 1900-1979, Croom Helm, 1st Edition, 1980.

[39] J. Grimmer, "A Bayesian Hierarchical Topic Mode for Political Texts: Measuring Expressed Agendas in Senate Press Releases," Political Analysis, vol. 18, no. 1, pp. 1-35, January 2010.

[40] J. H. Michael, "Labor Dispute Reconciliation in a Forest Products Manufacturing Facility," Forest Products Journal, vol. 47, no. 11-12, pp. 41-45, October 1997.

[41] E. Schwimmer, Exchange in the Social Structure of the Orokaiva: Traditional and Emergent Ideologies in the Northern District of Papua, C Hurst and Co-Publishers Ltd., December 1973.

[42] M. Pearson and L. Michell, "Smoke Rings: Social Network Analysis of Friendship Groups, Smoking and Drug-taking," Drugs: Education, Prevention and Policy, vol. 7, no. 1, pp. 21-37, 2000.

[43] T. Nepusz, A. Petroczi, L. Negyessy and F. Bazso, "Fuzzy Communities and the Concept of Bridgeness in Complex Networks," Physical Review E, vol. 77, no. 1, 016107, January 2008.

[44] N. Meghanathan, " Complex Network Analysis of the Contiguous United States Graph," Computer and Information Science, vol. 10, no. 1, pp. 54-76, February 2017.

[45] L. C. Freeman, C. M. Webster and D. M. Kirke, "Exploring Social Structure using Dynamic ThreeDimensional Color Images," Social Networks, vol. 20, no. 2, pp. 109-118, April 1998.

[46] L. C. Freeman, S. C. Freeman and A. G. Michaelson, "How Humans See Social Groups: A Test of the Sailer-Gaulin Models," Journal of Quantitative Anthropology, vol. 1, pp. 229-238, 1989.

[47] D. A. Smith and D. R. White, "Structure and Dynamics of the Global Economy: Network Analysis of International Trade 1965-1980," Social Forces, vol. 70, no. 4, pp. 857-893, June 1992.

[48] K. P. Balanda and H. L. MacGillivray, "Kurtosis: A Critical Review," The American Statistician, vol. 42, no. 2, pp. 111-119, 1988.

[49] J. H. Livesey, "Kurtosis Provides a Good Omnibus Test for Outliers in Small Samples," Clinical Biochemistry, vol. 40, no. 13-14, pp. 1032-1036, September 2007.

[50] K. Goh, E. Oh, C-M. Ghim, B. Kahng and D. Kim, "Classes of the Shortest Pathway Structures in Scale Free Networks," Complex Networks, Lecture Notes in Physics, vol. 650, pp. 105-125, August 2004. 


\title{
DYNAMIC OUTPUT FEEDBACK CONTROL FOR SAMPLED-DATA SYSTEM WITH ACTUATOR SATURATION USING QUANTIZED MEASUREMENTS
}

\author{
Liuwen $\mathrm{Li}^{1}$, Wenlin $\mathrm{Zou}^{2}$ and Shumin $\mathrm{Fei}^{1}$ \\ ${ }^{1}$ School of Automation, Southeast University, Nanjing, China \\ ${ }^{2}$ School of Computer Science and Engineering, Nanjing University of Science \\ and Technology, Nanjing, China
}

\begin{abstract}
This paper is focused on the dynamic output feedback control of sampled-data system with actuator saturation using quantized measurements. The output signals are quantized before they are transmitted to dynamic output-feedback controller. An augmented system is constructed through a closed-loop system, which is a model with an interval time-varying delay and nonlinear items. Using discontinuous Lyapunov function and linear matrix inequality approach, the criterion of asymptotical stability is obtained; furthermore, on the basis of the above stability condition, we design a dynamic output-feedback controller. Lastly, the effective of the proposed method is proved by a numerical example.
\end{abstract}

\section{KEYWORDS}

Discontinuous Lyapunov function, Dynamic output-feedback control, Sampled-data control systems, Saturations, quantization.

\section{INTRODUCTION}

For several decades, the stability of control system has been researched by many researchers because of its wide usage in many fields. In recent years, more and more control problems turn to digital controllers to solve. The key point of this control method is at discrete time but not continuous time of the state variable samples. Therefore, sampled-data control systems $[15,16,18$, 19] have attracted much attention. Three main approaches have been used to research the sampled-data control of linear systems. The first approach is discrete-time models [1,20]. The second one is impulsive model [3]. And the third is time-delay model which is via constructing Lyapunov-Razumikhin functions or Lyapunov-Krasovskii functional and using LMIs analyze the stability and design the controller of sampled-data control systems [2, 4, 15, 22, 23].

Saturation is very common in practical application because of the technological or safety constraints. Considering the importance of the saturation, researchers have studied saturation with the problem of filtering $[5-9,20]$ and the problem of control [6-8, 10, 21]. Just as saturation, control using quantized feedback has been a hot research topic for many years. Kalman studied the effect of quantization in a sampled data system in 1956 and presented that the feedback system would exhibit limit cycles and chaotic behaviour if a stabilizing controller is quantized using a Natarajan Meghanathan et al. (Eds) : ACSIT, SIPM, FCST, CoNeCo, CMIT - 2017 
finite-alphabet. [25] studied a number of quantized feedback design problems for linear systems and the main discovery is that the classical sector bound method is non conservative for studying the design problems. The problem of quantized state-feedback control has been investigated through a quantization dependent approach in [26] and new stability and stabilization conditions have been given. Quantization has also emerged in many literatures, such as in[8], [24], [27]. In this paper, we develop the static output-feedback in [15] to dynamic output-feedback control system under saturation and quantization constraint.

This paper concentrates on asymptotical stability of the sampled data control system under saturation and quantization constraint, controlled by dynamic output-feedback. Our aim is to analyze the performance of the closed loop system under above constraint. Firstly, a model of sampled-data system is constructed. There for, based on the discontinuous Lyapunov function and employing linear matrix inequality approach, sufficient conditions for system stability are obtained. Then, a dynamic output-feedback controller is designed on the basis of above stability. Finally, the practical utility of this method is proved by a numerical example.

The paper is organized as follows. Section 2 presents some useful lemmas and definition. Section 3 presents the system and the dynamic output-feedback controller we study. Section 4gives sufficient condition of system stability and dynamic output-feedback controller through linear matrix inequality approach. Section 5 illustrates the obtained results by a numerical example, which is followed by the conclusion in Section 6.

\section{LEMMAS AND DEFINITIONS}

In order to describe the system more clearly, we will give the following definitions and lemmas.

Lemma 1[12]The saturation function $\delta(u(t))$ can be decomposed into a linear and a nonlinear part as following

$$
\delta(u(t))=u(t)-\phi(u(t))
$$

and there exists a scalar $0<\varepsilon<1$, such that:

$$
\varepsilon u^{T}(t) u(t) \geq \phi^{T}(u(t)) \phi(u(t))
$$

Lemma2 [14] For matrix $\mathrm{R}>0$ and $X^{T}=X$, we have

$$
-X R^{-1} X \leq \rho^{2} R-2 \rho X
$$

Definition 1 A quantizer is called to be logarithmic if the set of quantization levels $\Pi$ can be described

$$
\Pi=\left[ \pm \pi_{i}: \pi_{i}=\rho^{i} \pi_{0}, \pi_{0}>0, i \in \square\right] \cup\{0\}, 0<\rho<1
$$


where $\rho$ is called the quantization density, besides, $\rho=\frac{1-k}{1+k}$, where $0<k<1$. Each of the quantization level corresponds to a segment such that the quantizer maps the whole segment to this quantization level. Precisely, the logarithmic quantization law of $q(\square)$ is defined as follows:

$$
q(w)=\left\{\begin{array}{lr}
v_{i}, \frac{1+\rho}{2} v_{i}<w \leq \frac{1+\rho}{2 \rho} v_{i}, & w>0, \\
0, & w=0, \\
-q(-w), & w<0 .
\end{array}\right.
$$

It can be found from [] and above Definition 1 that $q_{j}(\cdot)(j=1,2, \cdots m)$ can be expressed by $q_{j}\left(x_{j}\right)=\left(1+\Delta_{q_{j}}\left(x_{j}\right)\right) x_{j}$ for certain $\left|\Delta_{q_{j}}\left(x_{j}\right)\right| \leq \varsigma_{q_{j}}(j=1,2, \cdots m) \cdot \varsigma_{q_{j}}(j=1,2, \cdots m)$ are all positive constants. For simplicity, we denote $\Delta_{q_{j}}\left(x_{j}\right)$ as $\Delta_{q_{j}}$. Define

$$
\Delta_{q}=\operatorname{diag}\left\{\Delta_{q_{1}}, \Delta_{q_{2}}, \cdots \Delta_{q_{m}}\right\}
$$

Thus, $q(\cdot)$ can be expressed as

$$
q(x)=\left(I+\Delta_{q}\right) x
$$

Lemma 3 [17] Let $z(t) \in W[a, b)$ and $z(\mathrm{a})=0$. Then, for any $n \times n$ matrix $\mathrm{R}>0$ the following inequality holds:

$$
\int_{a}^{b} z(s) R z(s) d s \leq \frac{4(b-a)^{2}}{\pi^{2}} \int_{a}^{b} \dot{z}(s) R \dot{z}(s) d s
$$

Lemma4(S-procedure)Let $N=N^{\mathrm{T}}, H, E, F_{q}$ be real matrices with appropriate dimensions, for any $F_{q}$ which satisfying $F_{q}^{\mathrm{T}} F_{q} \leq I$. Then the inequality $N+H F E+(H F E)^{\mathrm{T}}<0$ if and only if there exists a positive scalar $\varepsilon$ such that $N+\varepsilon H H^{\mathrm{T}}+\varepsilon^{(-1)} E^{\mathrm{T}} E<0$, or, equivalently,

$$
\left[\begin{array}{ccc}
N & \varepsilon H & E^{\mathrm{T}} \\
\varepsilon H^{\mathrm{T}} & -\varepsilon I & 0 \\
E & 0 & -\varepsilon I
\end{array}\right]<0
$$

\section{Problem Formulation}

\subsection{Sampled-data system}

Consider thesampled-data system scheme as the following:

$$
\left\{\begin{array}{c}
\dot{x}(t)=A x(t)+B_{1} \delta_{u}(u(t)) \\
y(t)=C x(t)
\end{array}\right.
$$


where $x(t) \in \square^{n}$ is the state vector, $u(t) \in \square^{m}$ is the control input vector, $\delta_{u}(u(t))$ are denoted as actuator saturation function; $y(t) \in \square^{p}$ is the measuredoutput vector.

\subsection{Dynamic output-feedback controller}

We denote $t_{k}(k=0,1,2, \cdots)$ as the updating instant time of the Zero-Order-Hold(ZOH) and at the same time denote this updating signal at the sensor $t_{k}-\tau_{m}$. Supposing $t_{k+1}-t_{k} \leq h(k=0,1,2, \cdots)$, where $h$ is a positive scalar which is the largest sampling interval.

Defining $\tau(t)=t-\left(t_{k}-\tau_{m}\right), t_{k} \leq t<t_{k+1}$, Then, the delay $\tau(t)$ satisfies:

$$
\tau_{m} \leq t_{k}-\left(t_{k}-\tau_{m}\right) \leq \tau(t)<t_{k+1}-\left(t_{k}-\tau_{m}\right)<h+\tau_{m}=\tau_{M}
$$

In this paper, we propose to design a stabilizing dynamic output-feedback controller described by:

$$
\left\{\begin{array}{c}
\dot{x_{c}}(t)=A_{c} x_{c}(t)+B_{c} x_{c}(t-\tau(t))+C_{c} q\left(y_{s}(t)\right) \\
u(t)=D_{c} x_{c}(t)
\end{array}\right.
$$

where $x_{c} \in \mathrm{R}^{n_{c}}$ is the controller state vector, $y_{s}(t) \in \mathrm{R}^{p}$ is the controller input. $q\left(y_{s}(t)\right)$ represents thelogarithmicquantization of $y_{s}(t) . A_{c}, B_{c}, C_{c}$ and $D_{c}$ are matrices of appropriate dimensions which are to be determined, $\tau(t)$ is defined as above.

Through the above analysis, we have $y_{s}(t)=y\left(t_{k}\right) t_{k} \leq t<t_{k+1}, k=0,1,2, \cdots$, also we can derive that the sensor output has a form $y_{s}\left(t_{k}\right)=y\left(t_{k}-\tau_{m}\right)$, considering the behavior of the ZOH, we have $q\left(y_{s}(t)\right)=q\left(y\left(t_{k}-\tau_{m}\right)\right), \quad t_{k} \leq t<t_{k+1}, k=0,1,2, \cdots$. By the definition of $\tau(t)$ , we can derive: $y\left(t_{k}-\tau_{m}\right)=y(t-\tau(t))$. Based on the Definition 1,

$$
q\left(y_{s}(\mathrm{t})\right)=q(y(t-\tau(t)))=\left(I+\Delta_{q}\right) y(t-\tau(t))=\left(I+\Delta_{q}\right) C x(t-\tau(t))
$$

Based on Lemma 1, we have:

$$
\delta_{u}(u(t))=u(t)-\phi_{u}(u(t))=D_{c} x_{c}(t)-\phi_{u}(u(t))
$$

and there exists a scalar $0<\varepsilon_{1}<1$ satisfying:

$$
\varepsilon_{1} u^{T}(t) u(t)-\phi_{u}^{T}(u(t)) \phi_{u}(u(t)) \geq 0
$$

\subsection{Augmented system}

From above analysis, substituting (9) and (8) into (6) and (7) respectively, the closed-loop system with dynamic output-feedback control can be described as:

$$
\left\{\begin{array}{c}
\dot{x(t)}=A x(t)+B_{1} D_{c} x_{c}(t)-B_{1} \phi_{u}(u(t)) \\
\dot{x_{c}}(t)=A_{c} x_{c}(t)+B_{c} x_{c}(t-\tau(t))+C_{c}\left(I+\Delta_{q}\right) C x(t-\tau(t))
\end{array}\right.
$$


Set $\xi(t)=\left[\begin{array}{c}x(t) \\ x_{c}(t)\end{array}\right]$, and $\mathrm{A}:=\left[\begin{array}{cc}A & B_{1} D_{c} \\ 0 & A_{c}\end{array}\right], \mathrm{B}:=\left[\begin{array}{cc}0 & 0 \\ C_{c}\left(I+\Delta_{q}\right) C & B_{c}\end{array}\right], \mathrm{C}:=\left[\begin{array}{c}-B_{1} \\ 0\end{array}\right]$

Especially, we denote $\quad E:=\left[\begin{array}{ll}I & 0\end{array}\right], H:=\left[\begin{array}{ll}0 & I\end{array}\right]$

So, (11) can be written as

$$
\dot{\xi}(t)=\mathrm{A} \xi(t)+\mathrm{B} \xi(t-\tau(t))+\mathrm{C} \phi_{u}(u(t))
$$

and the inequality (10) can be described by:

$$
\varepsilon_{1} \xi^{\mathrm{T}}(t) H^{\mathrm{T}} D_{c}^{\mathrm{T}} D_{c} H \xi(t)-\phi_{u}^{\mathrm{T}}(u(t)) \phi_{u}(u(t)) \geq 0
$$

\section{MAIN RESULTS}

\subsection{SUFFICIENT CONDITIONS OF STABILITY}

In this section, we will give asymptotical control stability criteria for system (11) under saturation and quantization condition .

Theorem 1.For given parameters $\tau_{m}$ and $\tau_{M}, \tau_{M} \geq \tau_{m}>0$ and scalars $\varepsilon_{1} \in(0,1)$, the sampled-based closed-loop system (11) with saturation and quantization constraints is asymptotically stable if there exist matrices $P>0, M>0, Q_{i}>0, R_{i}>0(\mathrm{i}=1,2)$ and appropriate matrix $S$ which satisfy: $\left[\begin{array}{cc}R_{2} & S^{\mathrm{T}} \\ S & R_{2}\end{array}\right] \geq 0$

$$
\Pi_{1}=\left[\begin{array}{ccccccccc}
\Pi_{11} & P \mathrm{~B} & R_{1} & 0 & P \mathrm{C} & H^{T} D_{c}^{T} & \tau_{m} \mathrm{~A}^{T} & \tau_{M n} \mathrm{~A}^{T} & \tau_{M n} \mathrm{~A}^{T} \\
* & \Pi_{22} & \Pi_{23} & R_{2}-S^{T} & 0 & 0 & \tau_{m} \mathrm{~B}^{T} & \tau_{M n} \mathrm{~B}^{T} & \tau_{M n} \mathrm{~B}^{T} \\
* & * & \Pi_{33} & S^{T} & 0 & 0 & 0 & 0 & 0 \\
* & * & * & -Q_{2}-R_{2} & 0 & 0 & 0 & 0 & 0 \\
* & * & * & * & -I & 0 & \tau_{m} \mathrm{C}^{T} & \tau_{M n} \mathrm{C}^{T} & \tau_{M n} \mathrm{C}^{T} \\
* & * & * & * & * & -\left(\varepsilon_{1} I\right)^{-1} & 0 & 0 & 0 \\
* & * & * & * & * & * & -R_{1}^{-1} & 0 & 0 \\
* & * & * & * & * & * & * & -R_{2}^{-1} & 0 \\
* & * & * & * & * & * & * & * & -W^{-1}
\end{array}\right]<0
$$

where $\quad \tau_{M m}:=\tau_{M}-\tau_{m} \quad, \quad \Pi_{11}:=P \mathrm{~A}+\mathrm{A}^{T} P+Q_{1}-R_{1} \quad, \quad \Pi_{22}:=-2 R_{2}+S+S^{T}-\frac{\pi^{2}}{4} M$, $\Pi_{23}:=R_{2}-S+\frac{\pi^{2}}{4} M, \Pi_{33}:=Q_{2}-Q_{1}-R_{1}-R_{2}-\frac{\pi^{2}}{4} M$.

Proof: Construct a discontinuous Lyapunov-Krasovskii function for system (11) as

$$
V(t)=V_{1}(t)+V_{2}(t)+V_{3}(t)+V_{4}(t), t \in\left[t_{k}, t_{k+1}\right)
$$

In which $\quad V_{1}(t)=\xi^{\mathrm{T}}(t) P \xi(t), \quad V_{2}(t)=\int_{t-\tau_{m}}^{t} \xi^{\mathrm{T}}(s) Q_{1} \xi(s) d s+\int_{t-\tau_{M}}^{t-\tau_{m}} \xi^{\mathrm{T}}(s) Q_{2} \xi(s) d s$ 
$V_{3}(t)=\tau_{m} \int_{-\tau_{m}}^{0} \int_{t+\theta}^{t} \dot{\xi}^{\mathrm{T}}(s) R_{1} \dot{\xi}(s) d s d \theta+\left(\tau_{M}-\tau_{m}\right) \int_{-\tau_{M}}^{-\tau_{m}} \int_{t+\theta}^{t} \dot{\xi}^{\mathrm{T}}(s) R_{2} \dot{\xi}(s) d s d \theta$

$V_{4}(t)=\left(\tau_{M}-\tau_{m}\right)^{2} \int_{t_{k}-\tau_{m}}^{t} \xi^{\mathrm{T}}(s) M \xi(s) d s-\frac{\pi^{2}}{4} \int_{t_{k}-\tau_{m}}^{t-\tau_{m}}\left[\xi(s)-\xi\left(t_{k}-\tau_{m}\right)\right]^{\mathrm{T}} M\left[\xi(s)-\xi\left(t_{k}-\tau_{m}\right)\right] d s$

$V_{4}(t)$ can be decomposed as a discontinuous term $V_{4 a}(t)$ and a continuous one $V_{4 b}(t)$, that is to say:

$$
V_{4}(t)=V_{4 a}(t)+V_{4 b}(t)
$$

Let:

$$
\begin{gathered}
V_{4 a}(t)=\left(\tau_{M}-\tau_{m}\right)^{2} \int_{t_{k}-\tau_{m}}^{t-\tau_{m}} \xi^{\mathrm{T}}(s) M \xi(s) d s-\frac{\pi^{2}}{4} \int_{t_{k}-\tau_{m}}^{t-\tau_{m}}\left[\xi(s)-\xi\left(t_{k}-\tau_{m}\right)\right]^{\mathrm{T}} M\left[\xi(s)-\xi\left(t_{k}-\tau_{m}\right)\right] d s \\
V_{4 b}(t)=\left(\tau_{M}-\tau_{m}\right)^{2} \int_{t-\tau_{m}}^{t} \xi^{\mathrm{T}}(s) M \xi(s) d s
\end{gathered}
$$

According to Lemma 3, we can see $V_{4 a}(t) \geq 0$, and $V_{4 a}(t)$ vanishes at $t=t_{k}$. Besides, $V_{4 b}(t) \geq 0$. Thus,

$$
\lim _{t \rightarrow t_{k}^{-}}(V(t)) \geq\left(V\left(t_{k}\right)\right)
$$

The time derivative of $\dot{V}(t, \xi(t))$ along the trajectory of system (12) is

$$
\begin{gathered}
\dot{V}(t)=\dot{V}_{1}(t)+\dot{V}_{2}(t)+\dot{V}_{3}(t)+\dot{V}_{4}(t) \\
\dot{V}_{1}(t)=2 \xi^{\mathrm{T}}(t) P \dot{\xi}(t) \\
\dot{V}_{2}(t)=\xi^{\mathrm{T}}(t) Q_{1} \xi(t)-\xi^{\mathrm{T}}\left(t-\tau_{M}\right) Q_{2} \xi\left(t-\tau_{M}\right)+\xi^{\mathrm{T}}\left(t-\tau_{m}\right)\left(Q_{2}-Q_{1}\right) \xi\left(t-\tau_{m}\right) \\
\dot{V}_{3}(t)=-\tau_{m} \int_{t-\tau_{m}}^{t} \dot{\xi}^{\mathrm{T}}(s) R_{1} \dot{\xi}(s) d s-\left(\tau_{M}-\tau_{m}\right) \int_{t-\tau_{M}}^{t-\tau_{m}} \dot{\xi}^{\mathrm{T}}(s) R_{2} \dot{\xi}(s) d s \\
+\dot{\xi}^{\mathrm{T}}(t)\left[\tau_{m}^{2} R_{1}+\left(\tau_{M}-\tau_{m}\right)^{2} R_{2}\right] \dot{\xi}(t) \\
\dot{V}_{4}(t)=\left(\tau_{M}-\tau_{m}\right)^{2} \dot{\xi}^{\mathrm{T}}(t) M \dot{\xi}(t)-\frac{\pi^{2}}{4}\left[\xi\left(t-\tau_{m}\right)-\xi(t-\tau(t))\right]^{T} M\left[\xi\left(t-\tau_{m}\right)-\xi(t-\tau(t))\right]
\end{gathered}
$$

Using Jensen inequality, we can obtain

$$
-\tau_{m} \int_{t-\tau_{m}}^{t} \xi^{\mathrm{T}}(s) R_{1} \xi(s) d s \leq-\left[\xi(t)-\xi\left(t-\tau_{m}\right)\right]^{\mathrm{T}} R_{1}\left[\xi(t)-\xi\left(t-\tau_{m}\right)\right]
$$

When

$$
\left[\begin{array}{ll}
R_{2} & S^{\mathrm{T}} \\
S & R_{2}
\end{array}\right] \geq 0
$$

the following can be derived:

$$
\begin{aligned}
-\left(\tau_{M}-\tau_{m}\right) \int_{t-\tau_{M}}^{t-\tau_{m}} \dot{\xi}^{\mathrm{T}}(s) R_{2} \dot{\xi}(s) d s & \leq-\left[\xi\left(t-\tau_{m}\right)-\xi(t-\tau(t))\right]^{\mathrm{T}} R_{2}\left[\xi\left(t-\tau_{m}\right)-\xi(t-\tau(t))\right] \\
& -\left[\xi(t-\tau(t))-\xi\left(t-\tau_{M}\right)\right]^{\mathrm{T}} R_{2}\left[\xi(t-\tau(t))-\xi\left(t-\tau_{M}\right)\right] \\
& -2\left[\xi\left(t-\tau_{m}\right)-\xi(t-\tau(t))\right]^{\mathrm{T}} S^{\mathrm{T}}\left[\xi(t-\tau(t))-\xi\left(t-\tau_{M}\right)\right]
\end{aligned}
$$


Substituting (17)in (16) and combining (18) and (19) into consideration, and adding saturation condition (13), we have

$$
\dot{V}(t) \leq \eta^{T}(t) \Pi_{1} \eta(t)
$$

Where $\eta^{T}(t)=\left[\xi^{T}(t), \xi^{T}(t-\tau(t)), \xi^{T}\left(t-\tau_{m}\right), \xi^{T}\left(t-\tau_{M}\right), \phi_{u}^{T}(u(t))\right]$

If condition (14) is satisfied, it can be seen that the closed loop system (11) is asymptotically stable. This completes the proof.

\subsection{Explicit form of sufficient conditions}

In the following theorem we will give the explicit form of the desired DOFC parameter.

Theorem 2.Based on condition and result of theorem 1, for given parameters $\tau_{m}$ and $\tau_{M}$, $\tau_{M} \geq \tau_{m}>0$ and scalars $\varepsilon_{i} \in(0,1)(i=1,2)$,the sampled-based closed-loop system(11) with saturation and quantization constraints is asymptotically stable if there exist matrices $P>0, M>0, Q_{i}>0, R_{i}>0(\mathrm{i}=1,2)$ and appropriate matrix $S$ which satisfy:

$$
\Pi_{2}=\left[\begin{array}{ccc}
\bar{\Pi} & \Gamma_{1}^{\mathrm{T}} & \varepsilon_{2} \Gamma_{2}^{\mathrm{T}} \\
* & -\varepsilon_{2} I & 0 \\
* & * & -\varepsilon_{2} I
\end{array}\right]<0
$$

where $\Gamma_{1}=[\left(P \bar{C}_{c}\right)^{\mathrm{T}}, \overbrace{0, \cdots 0}^{5}, \tau_{m} \bar{C}_{c}^{\mathrm{T}},\left(\tau_{M}-\tau_{m}\right) \bar{C}_{c}^{\mathrm{T}},\left(\tau_{M}-\tau_{n}\right) \bar{C}_{c}^{\mathrm{T}}], \Gamma_{2}=[0, \bar{C}, \overbrace{0, \cdots 0}^{7}]$,

$$
\overline{\Pi_{1}}=\left[\begin{array}{ccccccccc}
\Pi_{11} & P \mathrm{~B}_{1} & R_{1} & 0 & P \mathrm{C} & H^{T} D_{c}^{T} & \tau_{m} \mathrm{~A}^{T} & \tau_{M n} \mathrm{~A}^{T} & \tau_{M n} \mathrm{~A}^{T} \\
* & \Pi_{22} & \Pi_{23} & R_{2}-S^{T} & 0 & 0 & \tau_{m} \mathrm{~B}_{1}^{T} & \tau_{M n} \mathrm{~B}_{1}^{T} & \tau_{M n} \mathrm{~B}_{1}^{T} \\
* & * & \Pi_{33} & S^{T} & 0 & 0 & 0 & 0 & 0 \\
* & * & * & -Q_{2}-R_{2} & 0 & 0 & 0 & 0 & 0 \\
* & * & * & * & -I & 0 & \tau_{m} \mathrm{C}^{T} & \tau_{M n} \mathrm{C}^{T} & \tau_{M n} \mathrm{C}^{T} \\
* & * & * & * & * & -\left(\varepsilon_{1} I\right)^{-1} & 0 & 0 & 0 \\
* & * & * & * & * & * & -R_{1}^{-1} & 0 & 0 \\
* & * & * & * & * & * & * & -R_{2}^{-1} & 0 \\
* & * & * & * & * & * & * & * & -W^{-1}
\end{array}\right]
$$

Where $\Pi_{11}, \Pi_{22}, \Pi_{22}, \Pi_{22}$ is the same as given in Theorem 1, and $\mathrm{B}_{1}=\left[\begin{array}{cc}0 & 0 \\ C_{c} C & B_{c}\end{array}\right]$.

Proof: First, we decompose: $\mathrm{B}=\mathrm{B}_{1}+\mathrm{B}_{0}$

$$
\begin{gathered}
\mathrm{B}_{1}=\left[\begin{array}{cc}
0 & 0 \\
C_{c} C & B_{c}
\end{array}\right], \mathrm{B}_{0}=\left[\begin{array}{cc}
0 & 0 \\
C_{c} \Delta_{q} C & 0
\end{array}\right]=\left[\begin{array}{c}
0 \\
C_{c}
\end{array}\right] F_{q}\left[\begin{array}{ll}
\bar{\Delta} C & 0
\end{array}\right] \\
\text { and in which } \Delta_{q}=F_{q} \bar{\Delta}, \quad F_{q}^{\mathrm{T}} F_{q} \leq I
\end{gathered}
$$




$$
\text { and simultaneously, we set } \bar{C}_{c}=\left[\begin{array}{c}
0 \\
C_{c}
\end{array}\right], \quad \bar{C}=\left[\begin{array}{ll}
\bar{\Delta} C & 0
\end{array}\right]
$$

$$
\text { Then, we can derive } \mathrm{B}_{0}=\bar{C}_{c} F_{q} \bar{C}
$$

Thus, we can rewrite $\Pi_{1}$ as the following form:

$$
\Pi_{1}=\overline{\Pi_{1}}+\Gamma_{1}^{\mathrm{T}} F_{q} \Gamma_{2}+\Gamma_{2}^{\mathrm{T}} F_{q}^{\mathrm{T}} \Gamma_{1}
$$

By utilizing Lemma 4,(26) is equivalent that there exist positive scalars $\varepsilon_{2}$ satisfying

$$
\Pi_{1}=\bar{\Pi}_{1}+\varepsilon_{2}^{-1} \Gamma_{1}^{\mathrm{T}} \Gamma_{1}+\varepsilon_{2} \Gamma_{2}^{\mathrm{T}} \Gamma_{2}
$$

And based on Schur complement, (21) is easy to obtain and the proof is completed.

\subsection{Dynamic output-feedback controller}

The following theorem presents the explicit parameters of the desired dynamic output feedback controller based on theorem 1 and theorem 2 .

Theorem 3.Based on condition and result of theorem 1and theorem 2, for given parameters $\tau_{m}$ and $\tau_{M}, \tau_{M} \geq \tau_{m}>0$ and scalars $\varepsilon_{i} \in(0,1)(i=1,2)$, the augmented system (12) is asymptotically stable there with system (11) is asymptotically stable if there exist matrices $P>0, M>0, Q_{i}>0, R_{i}>0(\mathrm{i}=1,2)$ and appropriate matrix $S$ which satisfy:

$$
\begin{aligned}
& {\left[\begin{array}{cc}
\tilde{R}_{2} & \tilde{S}^{T} \\
\tilde{S} & \tilde{R}_{2}
\end{array}\right] \geq 0, \quad Z:=\left[\begin{array}{cc}
X & I \\
* & P_{11}
\end{array}\right]>0} \\
& \text { and } \Pi_{3}=\left[\begin{array}{ccc}
\Pi & F_{1}^{\mathrm{T}} & \varepsilon_{2} \vec{F}_{2}^{\mathrm{T}} \\
* & -\varepsilon_{2} I & 0 \\
* & * & -\varepsilon_{2} I
\end{array}\right]<0
\end{aligned}
$$

where $\mathrm{F}_{1}=[\varphi_{5}{ }^{\mathrm{T}}, \overbrace{0, \cdots 0}^{5}, \tau_{m} \varphi_{5}{ }^{\mathrm{T}},\left(\tau_{M}-\tau_{m}\right) \varphi_{5}^{\mathrm{T}},\left(\tau_{M}-\tau_{n}\right) \varphi_{5}^{\mathrm{T}}], \mathrm{F}_{2}=[0, \varphi_{6}, \overbrace{0, \cdots 0}^{7}]$,

$$
\bar{\Pi}_{1}=\left[\begin{array}{ccccccccc}
\mathrm{H}_{11} & \varphi_{2} & R_{1} & 0 & \varphi_{3} & \varphi_{4} & \tau_{m} \varphi_{1}^{T} & \tau_{M n} \varphi_{1}^{T} & \tau_{M n} \varphi_{1}^{T} \\
* & \mathrm{H}_{22} & \mathrm{H}_{23} & R_{2}-S^{T} & 0 & 0 & \tau_{m} \varphi_{2}^{T} & \tau_{M n} \varphi_{2}^{T} & \tau_{M n} \varphi_{2}^{T} \\
* & * & \mathrm{H}_{33} & S^{T} & 0 & 0 & 0 & 0 & 0 \\
* & * & * & -Q_{2}-R_{2} & 0 & 0 & 0 & 0 & 0 \\
* & * & * & * & -I & 0 & \tau_{m} \varphi_{3}^{T} & \tau_{M n} \varphi_{3}^{T} & \tau_{M n} \varphi_{3}^{T} \\
* & * & * & * & * & -\left(\varepsilon_{1} I\right)^{-1} & 0 & 0 & 0 \\
* & * & * & * & * & * & R_{1}-2 Z & 0 & 0 \\
* & * & * & * & * & * & * & R_{2}-2 Z & 0 \\
* & * & * & * & * & * & * & * & W-2 Z
\end{array}\right]
$$


in which $\mathrm{H}_{11}=\varphi_{1}+\varphi_{1}^{T}+Q_{1}-R_{1}, \Pi_{22}=-2 R_{2}+S+S^{T}-\frac{\pi^{2}}{4} M, \Pi_{23}=R_{2}-S+\frac{\pi^{2}}{4} M$, $\Pi_{33}:=Q_{2}-Q_{1}-R_{1}-R_{2}-\frac{\pi^{2}}{4} M$

and $\varphi_{1}=\left[\begin{array}{cc}A X+B_{1} W_{1} & A \\ W_{4} & P_{11} A\end{array}\right], \varphi_{2}=\left[\begin{array}{cc}0 & 0 \\ W_{3} & W_{2} C\end{array}\right], \varphi_{3}=\left[\begin{array}{c}-B_{1} \\ -P_{11}^{T} B_{1}\end{array}\right]$,

$$
\varphi_{4}=\left[\begin{array}{c}
W_{1}^{T} \\
0
\end{array}\right], \quad \varphi_{5}=\left[\begin{array}{c}
0 \\
W_{2}
\end{array}\right], \quad \varphi_{6}=\left[\begin{array}{c}
X C^{T} \bar{\Delta}^{T} \\
C^{T} \bar{\Delta}^{T}
\end{array}\right]
$$

Proof: Since $P>0$, thus the matrix $P$ is nonsingular, then we partition $P$ as

$$
P=\left[\begin{array}{cc}
P_{11} & P_{12} \\
* & P_{22}
\end{array}\right]
$$

Where $P_{11}, P_{12}, P_{22} \in \mathrm{R}^{n \times n}$, what's more, it is easy to see $P_{11}>0$ and $P_{22}>0$. Without loss of generality, suppose $P_{12}$ is nonsingular square matrix. Define the matrix $Z$ which is satisfied $Z>0$, where $Z$ is defined. The relation of $P$ and $Z$ is

$$
\left[\begin{array}{cc}
I & P_{11} \\
0 & P_{12}^{T}
\end{array}\right]=P\left[\begin{array}{cc}
X & P_{12} \\
P_{12}^{-1}(I-P 11 X) & 0
\end{array}\right]
$$

Let

$$
L_{1}=\left[\begin{array}{cc}
X & P_{12} \\
P_{12}^{-1}\left(I-P_{11} X\right) & 0
\end{array}\right] L_{2}=\left[\begin{array}{cc}
I & P_{11} \\
0 & P_{12}^{T}
\end{array}\right]
$$

It is easy to know $L_{1}$ and $L_{2}$ are both non-singular.

Define $\mathrm{L}:=\operatorname{diag}\left\{L_{1}, L_{1}, L_{1}, L_{1}, I, I, L_{2}, L_{2}, L_{2}, I, I\right\}$. Performing congruence transformation to the matrix inequality (21), we get

$$
\Pi_{3}=\mathrm{L}^{T} \Pi_{2} \mathrm{~L}
$$

Denote $\tilde{S}=L_{1}^{T} S L_{1}, \tilde{M}=L_{1}^{T} M L_{1}, \tilde{Q}_{i}=L_{1}^{T} Q_{i} L_{1}, \tilde{R}_{i}=L_{1}^{T} R_{i} L_{1}(i=1,2)$. According to the denotation $\tilde{R}_{i}=L_{1}^{T} R_{i} L_{1}(i=1,2)$, we can derive

$$
R_{i}^{-1}=L_{1} \tilde{R}_{i}^{-1} L_{1}^{T}
$$

In the computational process, we can get the item $-L_{2}{ }^{T} R_{i}^{-1} L_{2}$, substituting (22) in it, we can obtain

$$
-L_{2}^{T} R_{i}^{-1} L_{2}=-L_{2}^{T} L_{1} \tilde{R}_{i}^{-1} L_{1}^{T} L_{2}=-Z^{T} \tilde{R}_{i}^{-1} Z
$$

By using Lemma 2, we have

$$
-Z^{T} \tilde{R}_{i}^{-1} Z \leq \tilde{R}_{i}-2 Z
$$

Similarly, we can easily obtain 


$$
-Z^{T} \tilde{M}^{-1} Z \leq \tilde{M}-2 Z
$$

and we define $W_{i}(\mathrm{i}=1,2,3,4)$ as

$$
\left\{\begin{array}{l}
W_{1}:=D_{c} P_{12}^{-1}\left(I-P_{11} X\right), W_{2}:=P_{12} C_{c} \\
W_{3}:=W_{2} C X+P_{12} B_{c} P_{12}^{-1}\left(I-P_{11} X\right) \\
W_{4}:=P_{11}^{T} A X+P_{11}^{T} B_{1} W_{1}+P_{12} A_{c} P_{12}^{-1}\left(I-P_{11} X\right)
\end{array}\right.
$$

It can be seen that all the process of proof is reversible, therefore, theorem 3 can also deduce Theorem 1 through Theorem 2, that is to say, the dynamic output feedback controller is:

$$
\left\{\begin{array}{l}
A_{c}=P_{12}^{-1}\left(W_{4}-P_{11}^{T} A X-P_{11}^{T} B_{1} W_{1}\right)\left(I-P_{11} X\right)^{-1} P_{12} \\
B_{c}=P_{12}^{-1}\left(W_{3}-W_{2} C X\right)\left(I-P_{11} X\right)^{-1} P_{12} \\
C_{c}=P_{12}^{-1} W_{2}, D_{c}=W_{1}\left(I-P_{11} X\right)^{-1} P_{12}
\end{array}\right.
$$

Remark 1: Though, the matrix of $P_{12}$ is unknown, we can see the DOFC in (7) is equivalent to the following DOFC in (41) by transforming $x_{c}(t)=P_{12}^{-1} \tilde{x}_{c}(t)$ and setting

$$
\left(\tilde{A}_{c}, \tilde{B}_{c}, \tilde{C}_{c}, \tilde{D}_{c}\right):=\left(P_{12} A_{c} P_{12}^{-1}, P_{12} B_{c} P_{12}^{-1}, P_{12} C_{c}, D_{c} P_{12}^{-1}\right) \text {, where } \tilde{A}_{c}, \tilde{B}_{c}, \tilde{C}_{c}, \tilde{D}_{c} \text { can be described as: }
$$

$$
\left\{\begin{array}{l}
\tilde{A}_{c}=\left(W_{4}-P_{11}^{T} A X-P_{11}^{T} B_{1} W_{1}\right)\left(I-P_{11} X\right)^{-1} \\
\tilde{B}_{c}=\left(W_{3}-W_{2} C X\right)\left(I-P_{11} X\right)^{-1} \\
\tilde{C}_{c}=W_{2} \\
\tilde{D}_{c}=W_{1}\left(I-P_{11} X\right)^{-1}
\end{array}\right.
$$

\section{NUMERICAL EXAMPLE}

In this section, a numerical example is presented to show the validity of the control approach for the sampled-data system with actuator saturation using quantized measurements.

Consider the system (6) described as:

$$
\left\{\begin{array}{c}
x(t)=\left[\begin{array}{ccc}
-2 & -0.1 & 0 \\
-0.1 & 0 & 0.2 \\
0 & -0.5 & 0
\end{array}\right] x(t)+\left[\begin{array}{c}
0.1 \\
0.02 \\
0.1
\end{array}\right] \delta_{u}(u(t)) \\
y(t)=\left[\begin{array}{lll}
1 & 0 & 0 \\
0 & 2 & 0
\end{array}\right] x(t)
\end{array}\right.
$$

In the following, our purpose is to design the DOFC in form (41)for the system (42), by setting $\varepsilon_{1}=0.2, \quad \varepsilon_{2}=0.6, \quad k=0.7, \quad \tau_{m}=0.02, \quad \tau_{M}=0.6$, the sampling interval $h=0.05$. Based on the Theorem 3 and Remark 1, we can obtain the corresponding DOFC parameters as:

$$
\tilde{A}_{c}=\left[\begin{array}{ccc}
-2.3132 & -21.3322 & -35.7622 \\
-0.0884 & -0.8822 & -1.1529 \\
0.0772 & -0.1137 & 0.0962
\end{array}\right], \quad \tilde{B}_{c}=\left[\begin{array}{rrr}
-0.0013 & 0.0172 & -0.0085 \\
0.0055 & -0.1918 & -0.0667 \\
-0.0005 & 0.0424 & 0.0394
\end{array}\right]
$$




$$
\tilde{C}_{c}=\left[\begin{array}{rr}
-0.0849 & 0.2176 \\
0.0255 & -1.5719 \\
-0.0318 & 0.1740
\end{array}\right], \quad \quad \tilde{D}_{c}=\left[\begin{array}{lll}
0.0124 & 1.0490 & 1.6772
\end{array}\right]
$$

When the initial condition is chosen as $x(0)=\left[\begin{array}{lll}2.6 & -1.5 & 1.8\end{array}\right]^{T}$, the responses of the state $x$ and the saturation control signal $\delta_{u}(u(t))$ are shown in Figures 1 and Figures 2, respectively. It can be seen that the closed-loop system is asymptotically stable. Simulation results indicate the effectiveness of the proposed control technology.

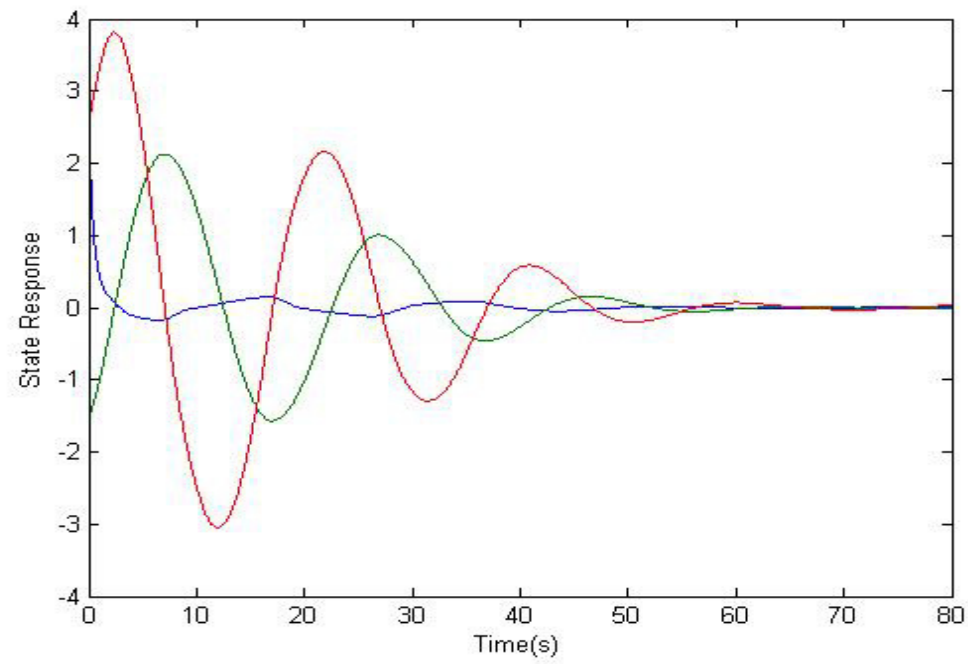

Figure 1. The state responses under DOFC

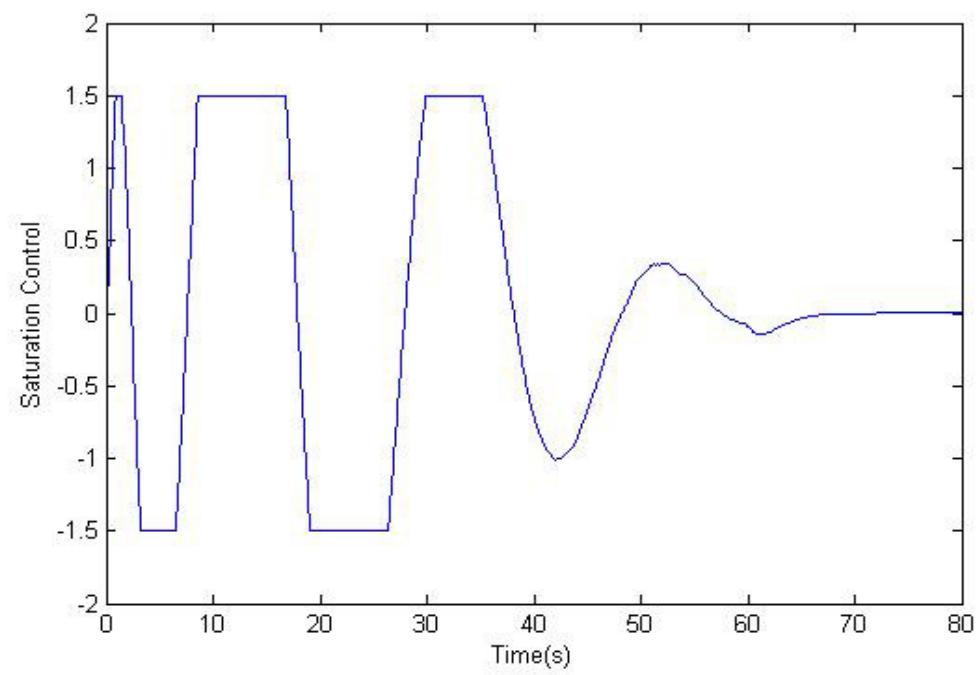

Figure 2. Output of saturation actuator 


\section{Conclusions}

In this paper, using discontinuous Lyapunov functional technique, we study the dynamic output feedback control of sampled-data system with saturation and quantization. A time-varying delay model with nonlinear items is represented as the closed-loop system, and a novel stability criterion is established by discontinuous Lyapunov functional approach. Sufficient conditions for asymptotic stability are derived employing linear matrix inequality approach and the corresponding matrix of DOFC are both designed. A numerical example shows that the results obtained in this paper are very practicable in analyzing the stability of the sampled data control system.

\section{ACKNOWLEDGEMENTS}

This work was supported by the Natural Science Foundation of China (NSFC) (No. 61273119, No. 61305011), Natural Science Foundation of Jiangsu Province of China (No. BK20150793), Plan of Policy Guidance in Jiangsu Province(No. BY2015004-11), and Jiangsu Agricultural Science and Technology Independent Innovation Fund Project (No. CX(15)1051).

\section{REFERENCES}

[1] W. Zhang. M. Branicky \& S. Phillips, (2001) "Stability of networked control systems”, IEEE Control System Magazine, Vol. 21, No. 1, pp84-99.

[2] E. Fridman, (2010) “A refined input delay approach to sampled-data control”, Automatica, Vol. 46, No. 2, pp421-427.

[3] H. Wang. Y, Ying \& R. Lu, (2016) "Network-based H-infinity control for singular systems with event-triggered sampling scheme", Information Sciences, Vol. 329, No. 1, pp540-551.

[4] D. Yue, E. Tian\&Q. Han, (2013) "A delay system method for designing event-triggered controllers of networked control systems", IEEE Transactions on Automatic Control, Vol. 58, No. 2, pp475-481.

[5] F. Yang \& Y. Li, (2009) "Set-membership filtering for systems with sensor saturation", Automatica, Vol. 45, No. 8, pp1896-1920.

[6] Z. Wang,B. Shen \& X. Liu, (2012) “ filtering with randomly occurring sensor saturations and missing measurements", Automatica, Vol. 48, No. 3, pp556-562.

[7] Z. Wang, W. Daniel, H. Daniel \& H. Gao, (2010) “ finite-horizon control for a class of stochastic nonlinear time-varying systems subject to sensor and actuator saturations", IEEE Transactions on Automatic Control, Vol. 55, No. 7, pp1716-1722.

[8] Y. Wei, M. Liu \& P. Shi, (2012) “ filtering for nonlinear stochastic systems with sensor saturation, quantization and random packet losses”, Signal Processing, Vol. 92, No. 6, pp1387-1396.

[9] T. Shi, H. Su \& J. Chu, (2013) "Reliable filtering for linear systems with sensor saturation", Journal of Control Theory and Applications, Vol. 11, No. 1, pp80-85.

[10] Z. Gu, S. Fei, Y. Zhao \& E. Tian, (2014) "Robust control of automotive active seat-suspension system subject to actuator saturation", Journal of Dynamic Systems, Measurement, and Control, Vol. 136, No. 4, pp041022(1)-041022(7).

[11] G. Garcia, S. Tarbouriech \& M. da Silva, (2007) "Dynamic output controller design for linear systems with actuator and sensor saturation”, IEEE American Control Conference, pp5834-5839.

[12] C. Peng \& M. Fei, (2013) "An improved result on the stability of uncertain T-S fuzzy systems with interval time-varying delay”, Fuzzy Sets and Systems, Vol. 212, No. 1, pp97-109. 
[13] L. Sun, Y. Wang \& G. Feng, (2015) "Control design for a class of affine nonlinear descriptor systems with actuator saturation", Transactions on Automatic Control, Vol. 60, No. 8, pp2195-2200.

[14] J. Xiong \& L. James, (2009) "Stabilization of networked control systems with a logic ZOH", IEEE Transactions on Automatic Control, Vol. 54, No. 2, pp358-363.

[15] K. Liu \& E. Fridman, (2012) "Wirtinger's inequality and Lyapunov-based sampled-data stabilization", Automatica, Vol. 48, No. 1, pp102-108.

[16] X. Liu, W. Yu, J. Cao \& S. Chen, (2015) "Discontinuous Lyapunov approach to state estimation and filtering of jumped systems with sampled-data”, Neural Networks, Vol. 68, No. 1, pp12-22.

[17] K. Liu, V. Suplin \& E. Fridman, (2010) "Stability of linear systems with general sawtooth delay", IMA Journal of Mathematical Control and Information, Vol. 27, No. 4, pp419-436.

[18] S. Lakshmanan, J. Park, R. Rakkiyappan \&H. Jung, (2013) "State estimator for neural networks with sampled data using discontinuous Lyapunov functional approach", Nonlinear Dynamics, Vol. 73, No. 1-2, pp509-520.

[19] Z. Chen, K. Shi \& S. Zhong, (2016) "New synchronization criteria for complex delayed dynamical networks with sampled-data feedback control”, ISA Transactions, Vol. 63, pp154-169.

[20] E. Tian, W. Wong, D. Yue \& T Yang, (2015) “ Filtering for Discrete-Time Switched Systems with Known Sojourn Probabilities”, IEEE Transactions on Automatic Control, Vol. 60, No. 9, pp2446-2451.

[21] E. Tian \& D. Yue, (2015) "Decentralized control of network-based interconnected systems: A state-dependent triggering method", International Journal of Robust and Nonlinear control, Vol. 25, No. 8, pp1126-1144.

[22] C. Peng \& Q. Han, (2016) "On designing a novel self-triggered sampling scheme for networked control systems with network-induced delays and data dropouts”, IEEE Transactions on Industrial Electronics, Vol. 63, No. 2, pp1239-1248.

[23] C. Peng, Y. Song, X. Xie, M. Zhao \& M. Fei, (2016) “An event-triggered output tracking control for wireless networked control systems with communication delays and data dropouts", IET Control Theory and Applications, Vol. 10, No. 17, pp2195-2203.

[24] E. Tian, D. Yue \& C. Peng, (2008) "Quantized output feedback control for networked control systems", Information sciences, Vol. 178, No. 12, pp2734-2749.

[25] M, Fu\& L Xie, (2005) "The sector bound approach to quantized feedback control", IEEE Transactions on Automatic control, Vol. 50, No. 11, pp1698-1711.

[26] H, Gao \& TChen, (2008) "A new approach to quantized feedback control systems", Automatica, Vol. 44, No. 2, pp534-542.

[27] S. Yun, J. Yun \& PPark, (2010) "Dynamic output-feedback guaranteed cost control for linear systems with uniform input quantization”, Nonlinear Dynamics, Vol. 62, No. 1, pp95-104. 


\section{AUTHORS}

\section{Liuwen Li}

received the M.S.degree in College of Science from Nanjing University of Aeronautics and Astronautics, Nanjing, China, in 2008. She is currently a PhD student of School of Automation Southeast University, Nanjing, China .Her current research includes time-delay systems and networked control systems, etc.

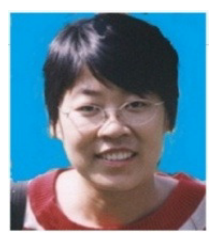

\section{WenlinZou}

received his Ph.D. degree in engineering from School of Automation, Southeast University, Nanjing, China, in 2014. Presently, he is doing postdoctoral research at School of Computer Science and Engineering, Nanjing University of Science and Technology, Nanjing, China. His current research interests include neural networks, time-delay systems, networked control systems, etc.

\section{Shumin Fei}

received the Ph.D. degree from Beijing University of Aeronautics and Astronautics in 1995, China. From 1995 to 1997, he was doing postdoctoral research at Research Institute of Automation, Southeast University, China. Presently, he is a professor and doctor adviser at Southeast University in China. He has published more than 70 journal papers and his current research interests include nonlinear systems, time-delay system, complex systems, and so on.
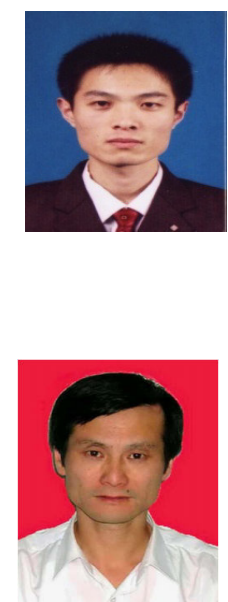


\title{
SECURING INFORMATION SYSTEMS A NEW APPROACH
}

\author{
William R. Simpson \\ Institute for Defense Analyses, 4850 Mark Center Dr., Alexandria, \\ Virginia 22311
}

\begin{abstract}
Increasing threat intrusions to enterprise computing systems have led to a formulation of guarded enterprise systems. The approach was to put in place steel gates and prevent hostile entities from entering the enterprise domain. The current complexity level has made the fortress approach to security implemented throughout the defence, banking, and other high trust industries unworkable. The alternative security approach presented in this paper is the result of a concentrated fourteen year program of pilots and research. Its distributed approach has no need for passwords or accounts and derives from a set of tenets that form the basic security model requirements. At each step in the process it determines identities and claims for access and privileges. These techniques are resilient, secure, extensible, and scalable. They are currently being implemented for a major enterprise, and are a candidate for other enterprise security approaches. This paper discusses the Enterprise Level Security architecture, a webbased security architecture designed to select and incorporate technology into a cohesive set of policies and rules for an enterprise information system. The paper discusses the history, theoretical underpinnings, implementation decisions, current status, and future plans for expansion of capabilities and scale.
\end{abstract}

\section{KEYWORDS}

Access control, attributes, authentication, claims, cryptography, digital signatures, enterprise, high assurance, identity management systems, public key infrastructure

\section{INTRODUCTION}

A set of problems exist where continued attempts at eliminating the symptom that results from design complexity has been unsuccessful at achieving the goal of eliminating the symptom.

\subsection{Case Study: Boat Design}

The original builders of boats had a simple design philosophy. Separate the water from the interior of the boat. In the beginning, boats were watertight by virtue of their being hewn from a single tree trunk, or when constructed from more than one piece of wood, joints were sealed with pitch or other sealants. That they leaked was not a big concern. The boats were not used in prolonged activity and did not get far from the shoreline. But as they were developed to move further offshore, some techniques were developed to prevent leaks (shiplap construction, for example) - they still leaked - just enough that it continually had to be dealt with. This limited their effectiveness and time before they needed to be brought ashore. As they got more complex, the leaks were of greater concern. Additional techniques were developed (tongue and grove construction, pitch and other sealants) - they still leaked. Sealants got better, but boats got more

Natarajan Meghanathan et al. (Eds) : ACSIT, SIPM, FCST, CoNeCo, CMIT - 2017 
complex as hatches for cargo, weapons, and steerable rudders mounted through the hull were added. Boats still leaked, not much, but just enough that it limited the range or speed, or time in the water without maintenance. Of course that wasn't going be tolerated! They doubled down (special woods, special formulated sealants, and special paints). They still leaked. A set of boat builders examined the history and came to an epiphany - boats leak. Like all epiphanies, it allows us to re-examine how we handle things. The design was modified so that the inside of boats could accommodate leakage. Drains and channels were added to funnel water to an area, the bilge, where the water could be dealt with, manually at first, and then with automated pumps. A well-sealed boat was still required because a high leakage rate could overwhelm bilge capacity.

\subsection{Case Study: Today}

We find ourselves at a crossroads where the computing systems we have come to rely on, are increasingly vulnerable to attack. Losses have occurred not only at commercial entities such as Target [1], Walmart [2], but at places that should be able to protect our information such as the IRS and FBI [3], OPM [4], etc. Who hasn't been hacked? The answer is surprising and it amounts to nobody. Nobody who is anybody and has something to lose, and somebody who is a nobody with limited presence and little or no assets online. How did we get in this mess?

\subsection{Case Study: Hindsight}

The Advanced Research Projects Agency Network (ARPAnet) was one of the world's first operational packet switching networks, the first network to implement TCP/IP, and the progenitor of what was to become the global Internet. The network was initially funded by the Advanced Research Projects Agency (ARPA, later the Defense Advanced Research Projects Agency (DARPA)) within the U.S. Department of Defense for use by its projects at universities and research laboratories in the United States. The packet switching of the ARPAnet, together with Transmission Control Protocol/Internet Protocol (TCP/IP), formed the backbone of how the Internet works. The packet switching was based on the concepts and designs of engineer Paul Baran, British scientist Donald Davies [5, 6], and Lawrence Roberts of Massachusetts Institute of Technology Lincoln Laboratory [7]. The TCP/IP set of communication protocols was developed for ARPAnet by computer scientists Robert Kahn and Vinton Cerf." [8]

The original ARPAnet connected four computers (1971). They were located in the respective computer research labs of the University of California, Los Angeles (UCLA) (Honeywell DDP 516 computer), Stanford Research Institute (SDS-940 computer), the University of California, Santa Barbara (IBM 360/75), and the University of Utah (DEC PDP-10). As the network expanded, different models of computers were connected, which created compatibility problems. The solution rested in a better set of protocols, TCP/IP, designed in 1982. Under ARPAnet, several major innovations occurred: email (or electronic mail) - the ability to send simple messages to another person across the network (1971); telnet-a remote connection service for controlling a computer (1972); and file transfer protocol (FTP)-allows information to be sent from one computer to another in bulk (1973).

As non-military uses for the network increased, more and more people had access, and it became no longer safe for military purposes. As a result, MILnet, a military-only network, was started in 1983. Internet Protocol software was soon being placed on every type of computer, and universities and research groups also began using in-house networks known as Local Area Networks or LANs. These in-house networks then started using Internet Protocol software so that one LAN could connect to another.

"In 1986, one LAN branched out to form a new competing network, the National Science Foundation Network (NSFnet). NSFnet first linked together the five national supercomputer centers and then every major university, replacing the slower ARPAnet, 
which was finally shut down in 1990. NSFnet formed the backbone of what we now call the Internet".

-A Brief History of Network Computing [9]

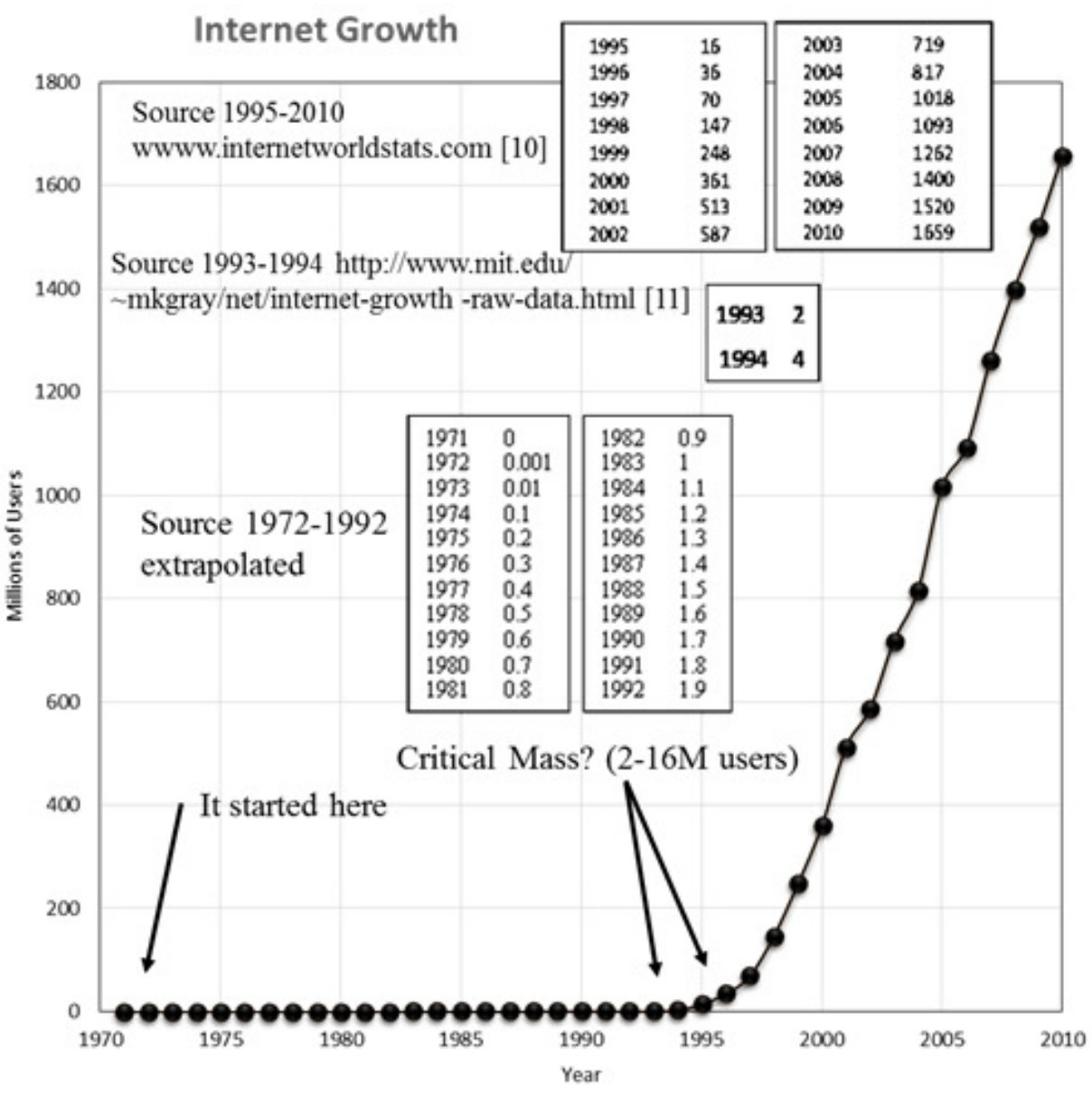

Figure 1. Internet Growth

In the beginning there were no threats only a desire to make computers communicate. This changed quickly. Intruders penetrated unprotected systems. The only thing that grew faster than the internet was the complexity of computing and the internet. Threats were few at first and targeted approaches were used to mitigate them. This worked for a while. Quickly however, more generic methods were needed.

\subsection{Case Study: The Fortress Approach}

The advent of the firewall is the beginning of the fortress approach.

"Acting as a barrier between a trusted network and other untrusted networks -- such as the Internet -- or less-trusted networks -- such as a retail merchant's network outside of a cardholder data environment -- a firewall controls access to the resources of a network through a positive control model. This means that the only traffic allowed onto the network defined in the firewall policy is; all other traffic is denied".

- Firewall [12] 
In the fortress approach a gateway is established that is hardened against all who would enter but were not authorized. This gateway is sometimes called the De-Militarized Zone (DMZ). While the DMZ began with the firewall it certainly didn't end there. Initially somewhat successful, the firewall quickly became ineffective against a number of attackers and the threat still penetrated.

The designers of the DMZ supplemented the DMZ with additional hardware "appliances". These were needed in hardware because they must process everything that comes to the enterprise and needed to operate at "line speeds" so as not to reduce the quality of service provided by the enterprise. The appliances may cost more than $\$ 1$ million each. They added packet inspection to the firewall - the threat still penetrated. Additional techniques (and appliances) were developed (Application aware filtering, white/black and grey listings, signature analysers) - the threat still penetrated. Of course that wasn't going be tolerated! They doubled down (Host based analysers, Intrusion detection, Intrusion prevention) - the threat still penetrated.

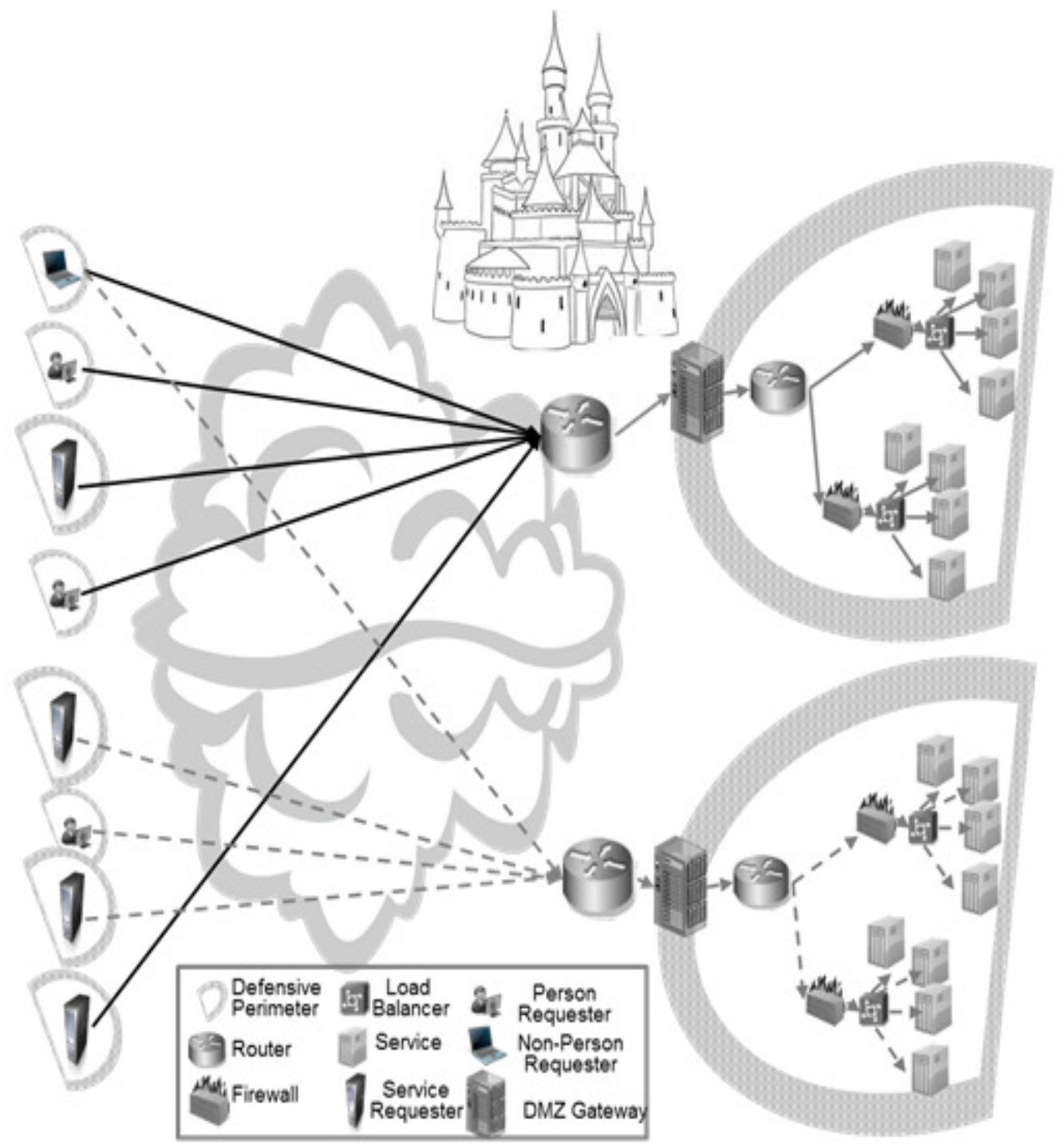

Figure 2. The Fortress Approach (DMZ) 


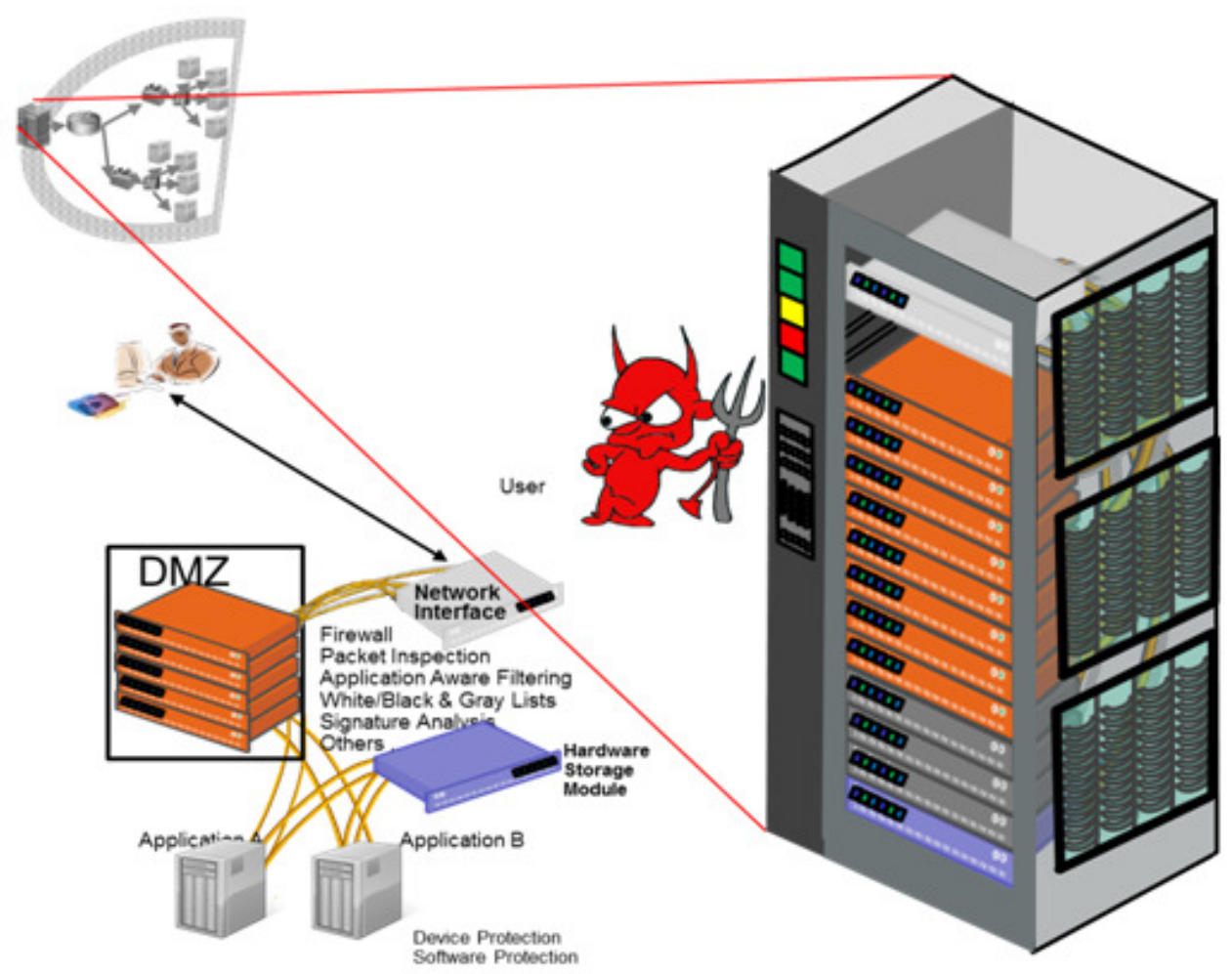

Figure 3. The "Beefed-up" DMZ

Does this sound familiar? It is time for an epiphany -threats penetrate systems. Like all epiphanies, it allows us to re-examine how we handle things.

\section{RE-EXAMINATION OF THE SECURITY APPROACH}

The complexity of modern systems has grown almost without bounds. Complexity makes the system only partially testable. Defence requires preventing everything. Offense requires finding one exploit. Threats cannot be eliminated, only mitigated. Adding complexity in defences exacerbates the problem. Complexity continues and will continue to grow!

The threat is part of the environment.

- Go back to security principles.

- Remove common sources of vulnerabilities (passwords, accounts, escalation of privilege ...).

- Replace passwords with credentials as a basis for trust. Verify and validate all credentials.

- Trust as little as possible. Minimize threat surfaces. Minimize the value of targets (distribute the value among targets).

- Communicate in confidentiality.

- Communicate endpoint to endpoint (no intermediates). Verify that what you received was what was sent (integrity).

- Know with whom you are dealing (no actions on behalf of).

- Know when you have been compromised.

- Monitor and record. Be resilient. ... 
Some basic assumptions have been made at the outset for the security model as derived from the re-examination above. These are:

- Only interactions based on authorization credentials and two-way, end-to-end authentication are permitted [this leads to strong requirements for enterprise naming and credentials];

- Impersonation is not allowed;

- Least Privilege;

- Confidentiality of all data/content exchanged;

- Verify and validate integrity of all communications;

- Monitoring is conducted on all exchanges;

- Eliminate or mitigate Malware by periodic scans and active measures.

The Enterprise Level Security (ELS) has evolved from a fortress approach, where threats are assumed to be stopped at the front door, to a distributed security system that eliminates or mitigates many of the primary vulnerability points within that system, as shown in Figure 4.

\section{Concentrate on the end points.}
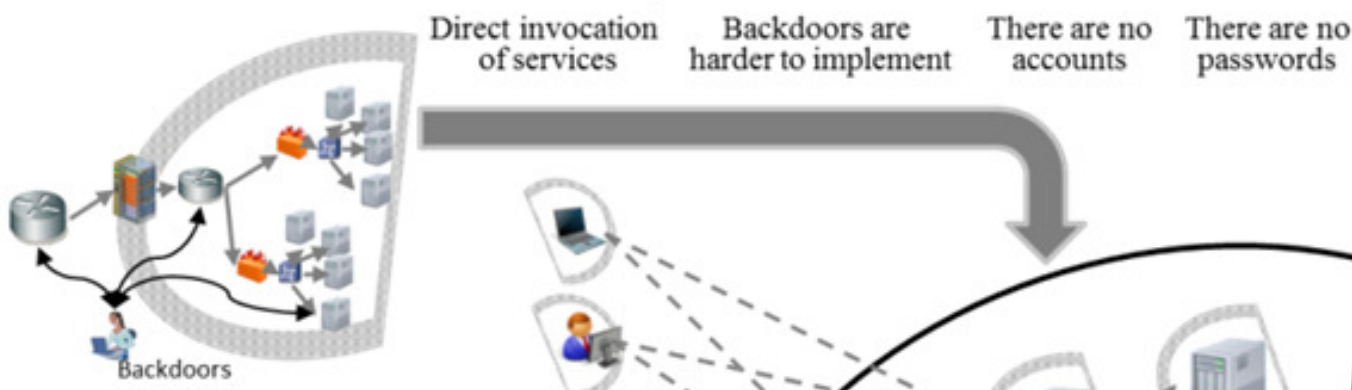

- Reduce the number of high value targets.

- By distributing even if you lose a battle the losses are confined to a small subset of the whole.

- Emphasize backup and recovery.

- Eliminate data warehouses.

- Incorporate federated databases.

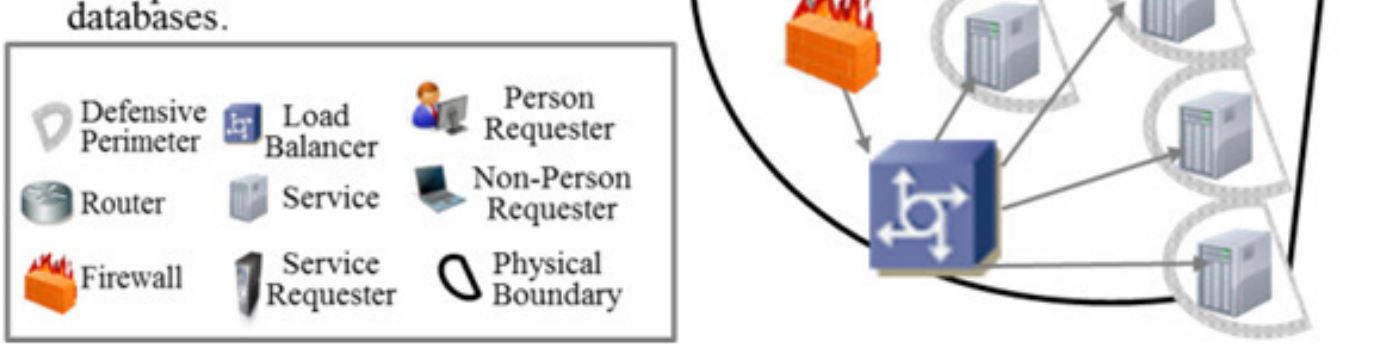

Figure 4. Distributed Security Architecture 


\section{ENTERPRISE LEVEL SECURITY}

ELS is a high-assurance environment. For ELS, we are primarily concerned with five security principles.

- Know the Players - this is done by enforcing bi-lateral end-to-end authentication.

- Maintaining Confidentiality - this entails end-to-end unbroken encryption.

- Separate Access and Privilege from Identity- this is done by an authorization credential

- Maintain Integrity - know that you received exactly what was sent - know that content has not been modified.

- Explicit Accountability - monitor all transactions.

\subsection{Know the Players}

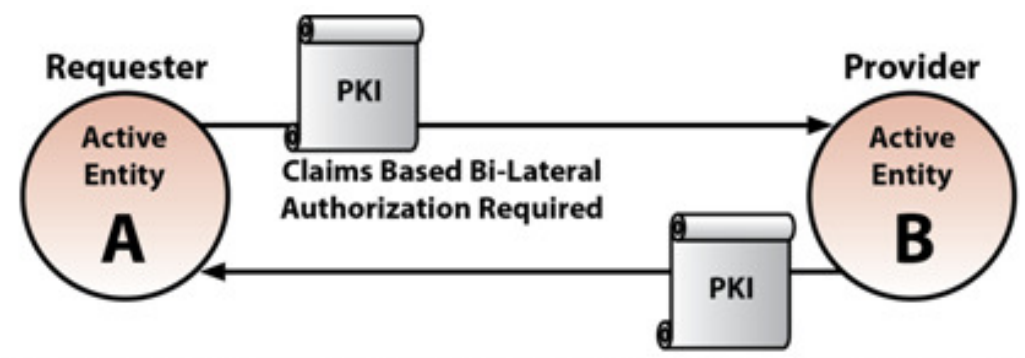

Active Entity may be: User, Web Application, Web Service, Aggregation Service, Exposure Service, Token Server, or any element that can be a requester or provider.

Figure 5. Bi-lateral Authentication

In ELS, the identity certificate is an X.509 PKI certificate [13]. PKI certificates are verified and validated. Ownership is verified by a holder-of-key check.

\subsection{Maintain Confidentiality}

ELS establishes end-to-end TLS [14] encryption (never give away private keys that belong uniquely to the certificate holder). Message authentication codes are enforced (but they are only valid when the encryption remains unbroken to the end point).

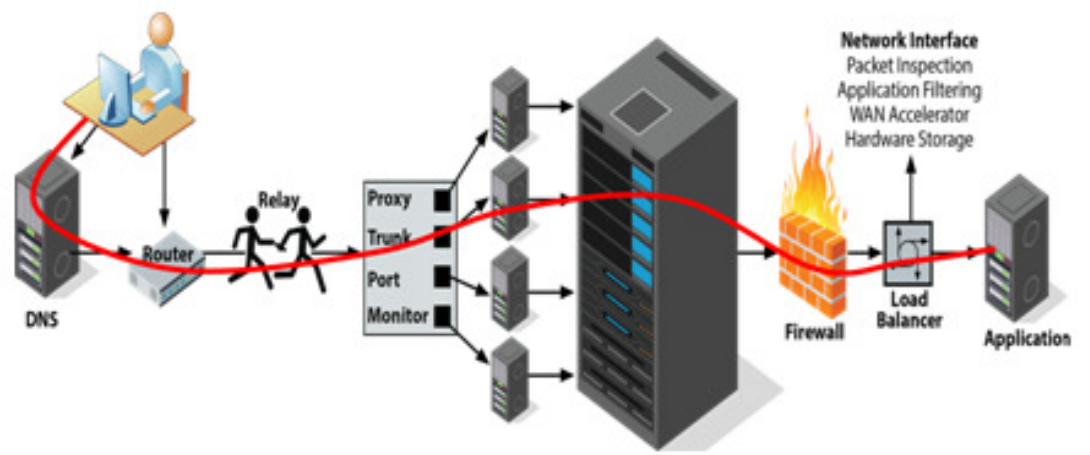

Figure 6. End-to-End Encryption 


\subsection{Separate Access and Privilege from Identity}

In ELS this is accomplished by using the Security Assertion Markup Language (SAML) [15]. SAMLs are signed, and the signatures are verified and validated. The credentials of the signers are verified and validated.

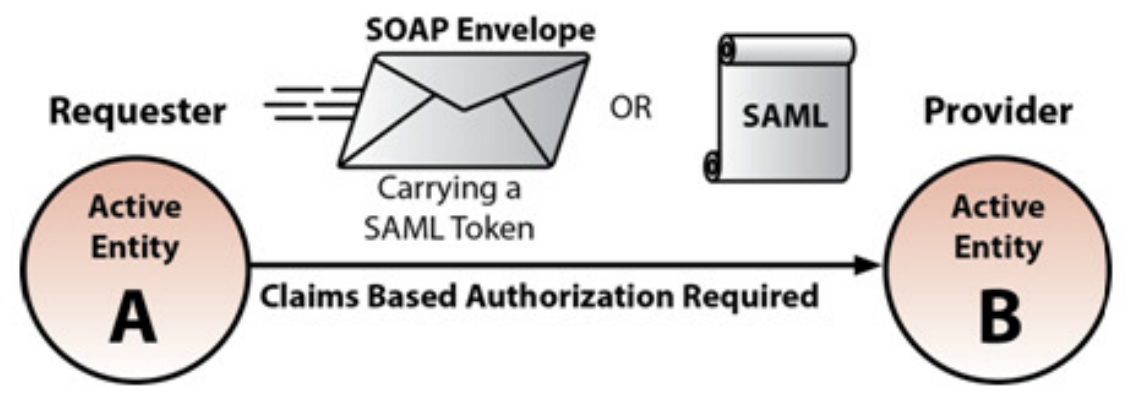

Figure 7. Claims-Based Authorization

\subsection{Maintain Integrity}

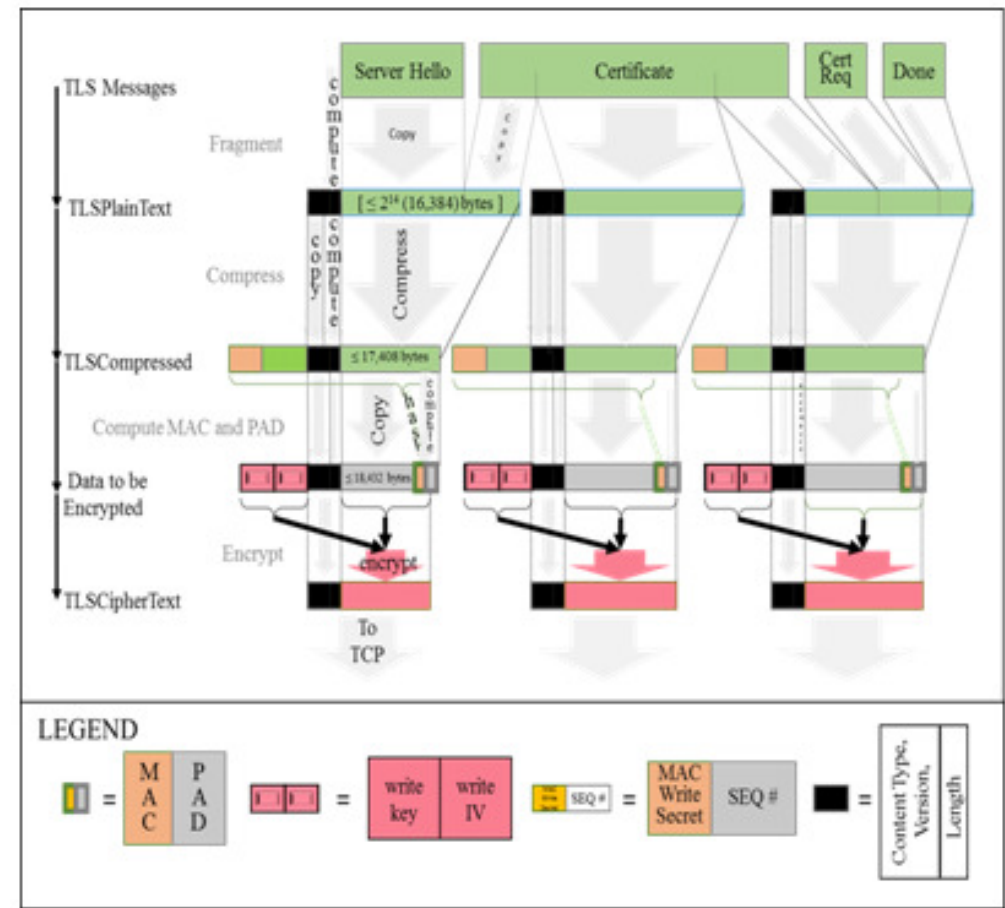

Figure 8. MAC and Other Integrity Measures

In ELS this is implemented by End-to-End TLS encryption with message authentication codes (MAC). Packages (like SAML tokens) are signed, and signatures are verified and validated [16].

\subsection{Require Explicit Accountability}

All active entities must monitor specified activities. For enterprise files a monitor sweep agent reads, translates, cleans, and submits to relational data base for recording log records periodically or on demand. The details of this activity are provided in [17 and 18]. 


\section{InSPECTIONS AND PRotections}

Most appliance functionality is now available as software only. Figure 10 shows the conversion of the particular piece of the inspection chain that applies to a particular server as a pseudo appliance.

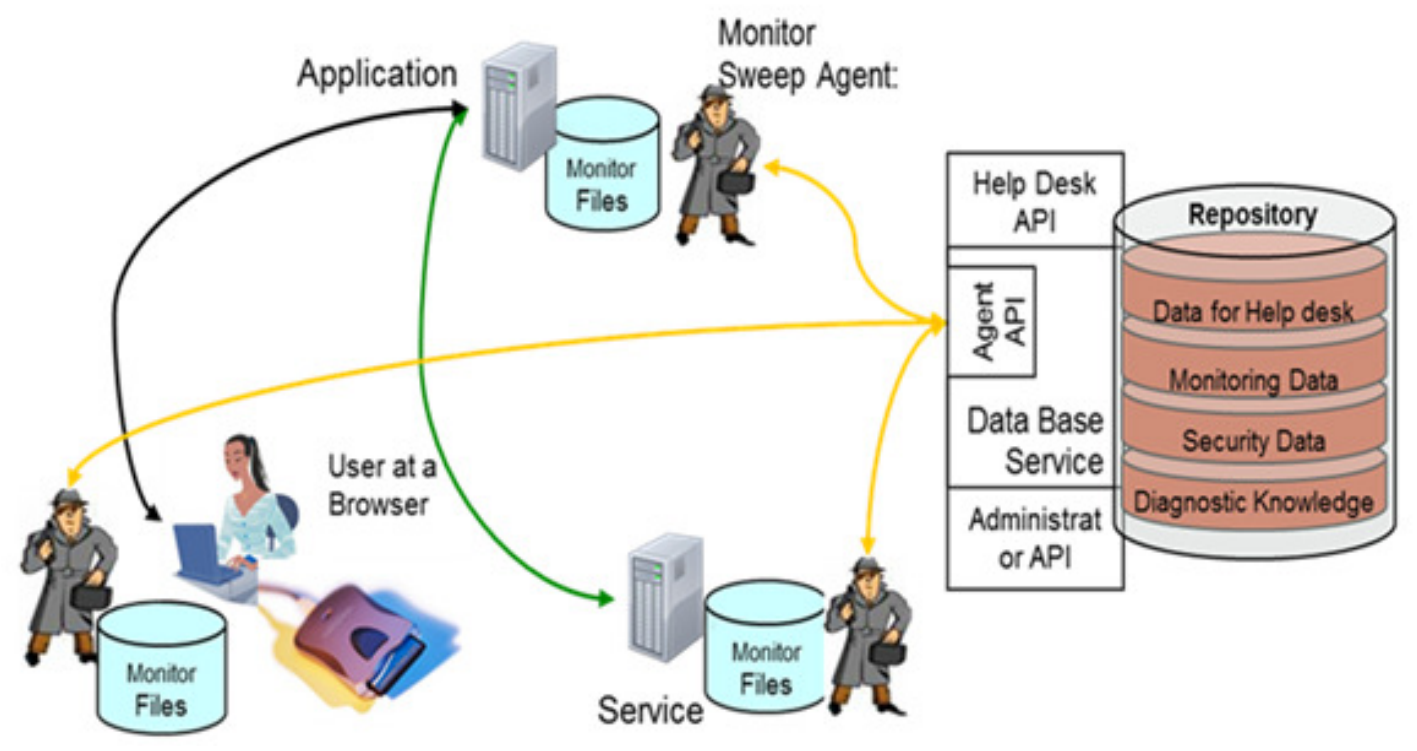

Figure. 9 Accountability through Centralized Monitoring

The packets are decrypted on entry into the pseudo appliance and stay that way with the exception of an offload to an external source (such as a network monitoring appliance where packets are counted and graded, etc. This offloading will be done by a full ELS communication (for security, of course). This sounds pretty similar to the current approach. How does this change anything? The difference is in where the pseudo appliance lives. For the moment let's assume the software appliance lives in the application server. The traffic at the server is considerably less than the front door of the enterprise (obviating the need for lightning speed and hardware only solutions). The inspections can also be tailored (closely inspect some, ignore others) for the specific application.

Of course we cannot send all of the incoming packets to the application server. It would be inefficient and dangerous. Some packets can wreak havoc before they are even processed, so we would want to be sure that the server was the intended recipient and that the server is communicating with a credentialed entity with valid credentials.

Figure 11 has identified an intelligent tagging device that will identify the traffic by observing the first few packets. In the case of official enterprise traffic the first few packets are not encrypted and these involve the exchange of PKI certificates that can be identified and the owners can be compared to a white list. The target will be the destination for traffic. Before identification the packets can be passed through the normal set of inspection appliances - sometimes referred to as the de-militarized zone (DMZ). If no identification is made, the packets will continue through the DMZ. When identified, they can be passed directly to the server or the load balancer in front of the server. 


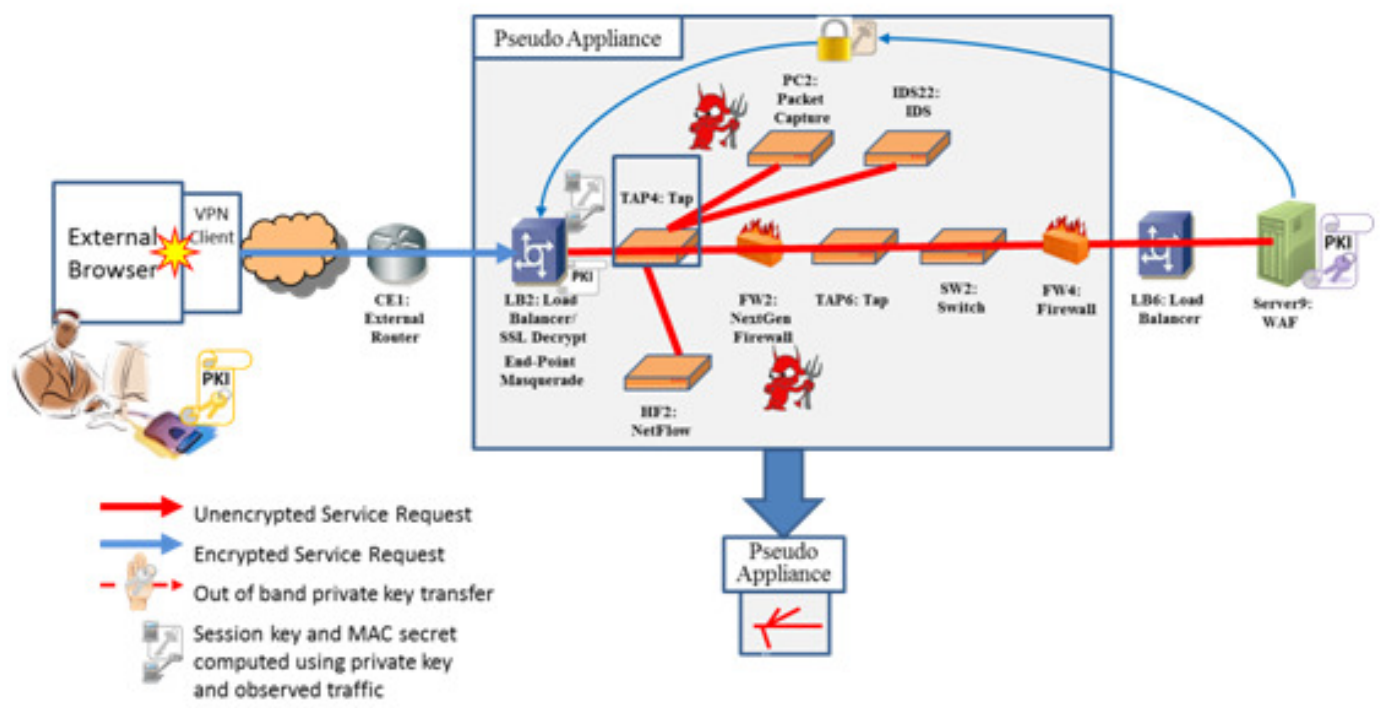

Figure 10. Creation of the Pseudo Appliance

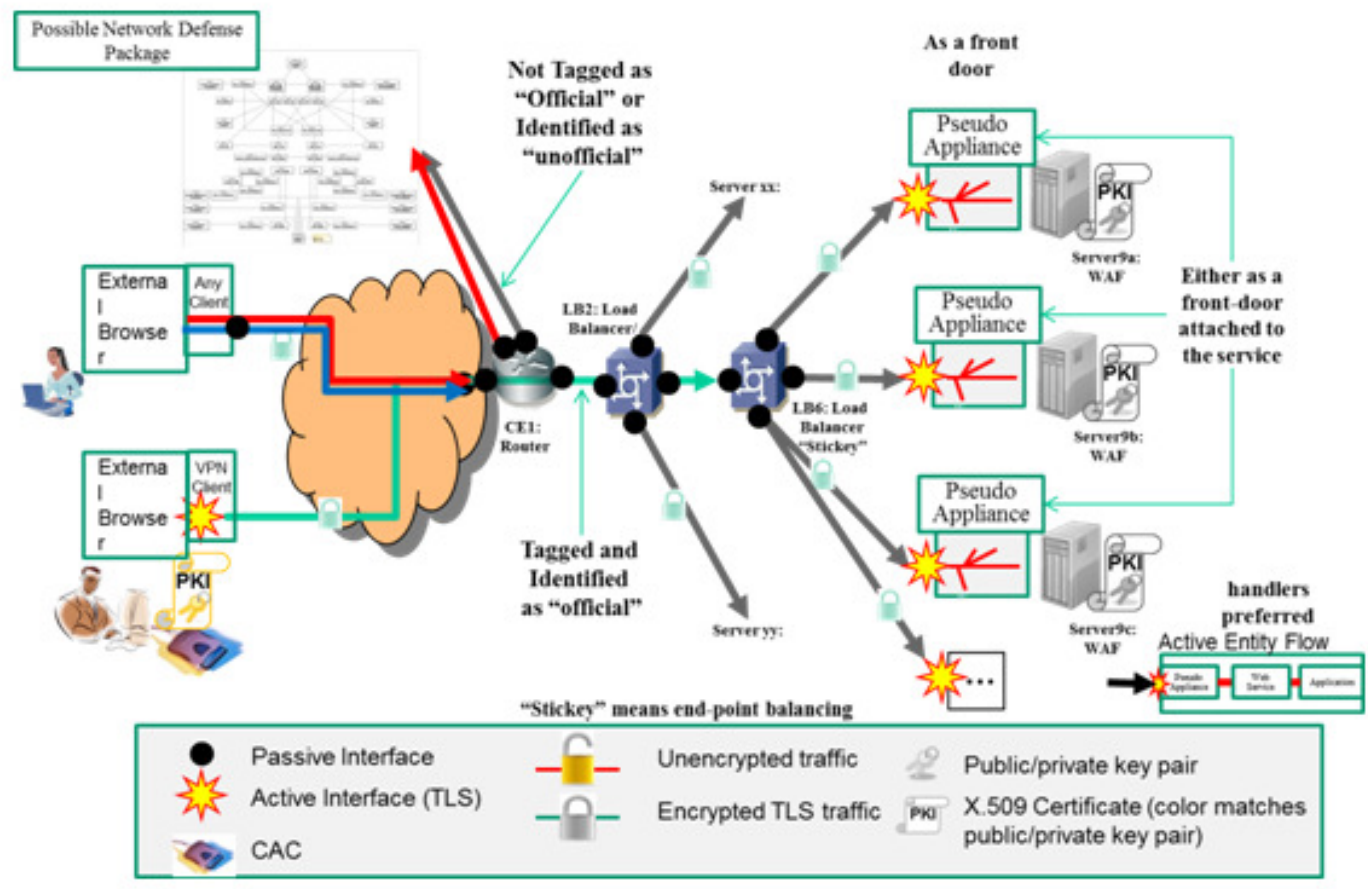

Figure 11. Tagged and Embedded Functionality

The only remaining problem is to reduce the software functionality to handlers in the handler chain as shown in Figure 12. 


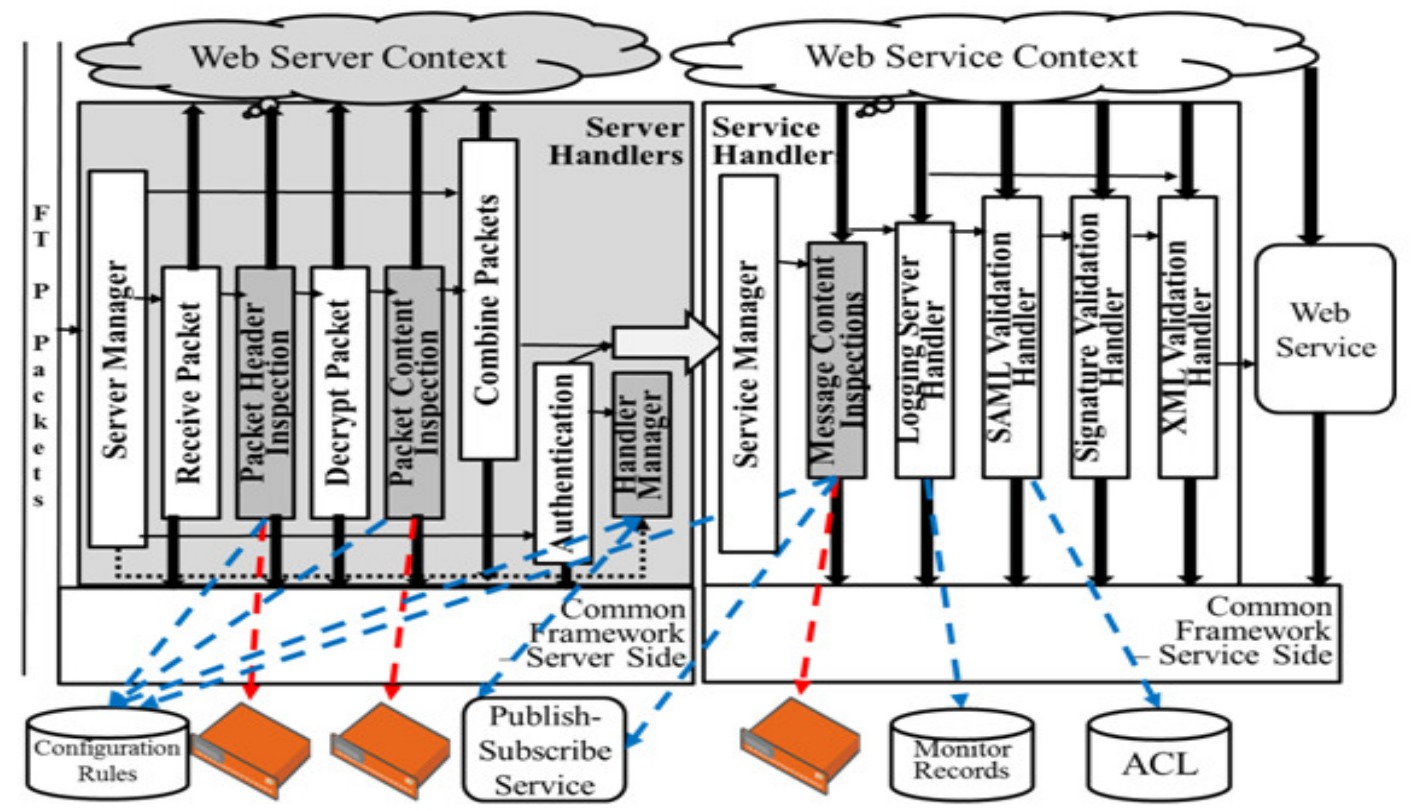

Figure. 12 Server Side Handlers

Note that the handlers are embedded in the server handler chain at the point that the communication is prepared for their use, and that the functionality has been divided along those lines as opposed to the previous functionality such as virus scan, ports and protocols, intrusion detection or blacklist/whitelist, etc. These are distributed to packet header inspection, packet content inspection, and message content inspection. Each of these may perform inspection related to intrusion detection or blacklist blocking, etc. Pilots are being worked on, stay tuned for results. This is the preferred embodiment for enterprise applications. It moves the inspections to the point of the application itself, by inserting handlers within the server and service to do the inspections at the point it makes most sense. The inspections that can be done without decrypting the packets may be done at the front of the web server because they are passive entities. Moving inspections of decrypted traffic inside the server, not only preserves the end-to-end paradigm, but encapsulates the security and allows tailoring for the application itself. The encapsulated security with the application is virtualization ready.

\section{An Evolution}

The ELS is the result of a carefully crafted architecture. The initial research began in 2002 with the original development of design tenets and each of the major components. These include:

- Fully encrypted unbroken end-to-end communications (later defined to be TLS with message authentication codes).

- Bi-lateral PKI authentication for all enterprise entities;

- SAML-based approaches as hardened for vulnerability mitigation for access and privilege;

- Embedded SAML handlers for consistency in application;

- Claims-based access and privilege approach as opposed to attributes and roles;

- Defined federation and delegation processes; 
- Virtualization inspection handles (in process).

A full implementation began in 2015 with a spiral based roll-out leading to pathfinder applications, Cyber range evaluations, and other applications currently in process.

\section{Conclusions}

We have reviewed the basic approaches to the restriction of database access, and the assignment of privilege with databases. The common approach to a web service front end of a Database Management System (DBMS) requires the web service to restrict access and privilege based upon the user context. In doing this it must be provided with full access and privilege to the database, and be trusted to limit user access and privilege. We reviewed the high-assurance security paradigm and the changes that must be made for hardening the security associated with database operations. The suggested approaches build increasing security by adding user-tailored restrictions directly into the database, and they provide the web service fronting the DBMS with the same privilege as the user. At the same time, it restricts SQL queries to a fundamental set that will be enforced by the view developed within the database and not at the web service. A final area, yet to be developed is the application of partial homomorphic techniques that keeps all transactions encrypted.

This research is part of a body of work for high-assurance enterprise computing using web services. Elements of this work include bi-lateral end-to-end authentication using PKI credentials for all person and non-person entities, a separate SAML credential for claims-based authorization, full encryption at the transport layer, and a defined federation process. Many of the elements of this work are described in [19-24]. The entire process has been recently published in [25]

\section{REFERENCES}

[1] Chris Poulin 2014, "What Retailers Need to Learn from the Target Breach to Protect against Similar Attacks”, https://securityintelligence.com/target-breach-protect-against-similar-attacks-retailers/ , accessed on 2/25/1016.

[2]. Kim Zetter 2009, "Big-Box Breach: The Inside Story of Wal-Mart's Hacker Attack", http://www.wired.com/2009/10/walmart-hack/, accessed on 2/25/1016.

[3]. Colleen Weller 2016, "Cyber-Security in 120 Secs: Breaches at FBI and IRS", http://blog.ensilo.com/cyber-security-in-120-secs-breaches-at-fbi-and-irs, accessed on 2/25/1016.

[4]. David Bisson 2015, “The OPM Breach: Timeline of a Hack", http://www.tripwire.com/state-ofsecurity/security-data-protection/cyber-security/the-opm-breach-timeline-of-a-hack/, accessed on $2 / 25 / 1016$.

[5]. Donald W. Davies CBE, FRS, June 7, 1924, Treorchy, UK, May 28, 2000, Australia, http://www.thocp.net/biographies/davies_donald.htm

[6]. INTERNET HALL of FAME PIONEER Donald Davies, http://www.internethalloffame.org/inductees/donald-davies

[7]. "Lawrence Roberts Manages the ARPANET Program." http://www.Living_Internet.com, Retrieved 6 November 2008.

[8]. ARPANET, http://en.wikipedia.org/wiki/ARPANET

[9]. A brief History of Network Computing, http://inventors.about.com/library/weekly/aa091598.htm

[10]. Internet Growth Data 1995-2010, www.internetworldstats.com 
[11]. MIT Internet Growth 1993-1994, http://www.mit.edu/ mkgray/net/internet-growth-raw-data.html, Internet Growth Data 1971-1992, Extrapolated by Author.

[12]. Essential Guide, "Enterprise firewall protection: Where it stands, where it's headed", http://searchsecurity.techtarget.com/definition/firewall, accessed on 2/25/1016.

[13]. X.509 Standards

a. DoDI 8520.2, Public Key Infrastructure (PKI) and Public Key (PK) Enabling, 24 May 2011

b. JTF-GNO CTO 06-02, Tasks for Phase I of PKI Implementation, 17 January 2006

c. X.509 Certificate Policy for the United States Department of Defense, Version 9.0, 9 February 2005

d. FPKI-Prof Federal PKI X.509 Certificate and CRL Extensions Profile, Version 6, 12 October 2005

e. RFC Internet X.509 Public Key Infrastructure: Certification Path Building, 2005

f. Public Key Cryptography Standard, PKCS \#1 v2.2: RSA Cryptography Standard, RSA Laboratories, October 27, 2012

g. PKCS\#12 format PKCS \#12 v1.0: Personal Information Exchange Syntax Standard, RSA Laboratories, June 1999 http://www.rsa.com/rsalabs/node.asp?id=2138 PKCS 12 Technical Corrigendum 1, RSA laboratories, February 2000

[14]. TLS family Internet Engineering Task Force (IETF) Standards

In draft for reference only:

h. TLS Renegotiation Support Extension to HTTP/2, 2015-03-24

i. Terminology related to TLS and DTLS, 2015-03-26

j. X.509v3 TLS Feature Extension, 2015-04-06

k. TLS over HTTP, 2015-03-09

1. A TLS ClientHello padding extension, 2015-02-17

m. Elliptic Curve Cryptography (ECC) Cipher Suites for Transport Layer Security (TLS) Versions 1.2 and Earlier, 2015-03-09

n. Transport Layer Security (TLS) Session Hash and Extended Master Secret Extension, 201504-16

o. The Transport Layer Security (TLS) Protocol Version 1.3, 2015-03-09

p. Standards:

q. RFC 2830 Lightweight Directory Access Protocol (v3): Extension for Transport Layer Security, 2000-05

r. RFC 3749 Transport Layer Security Protocol Compression Methods, 2004-05

s. RFC 4279 Pre-Shared Key Ciphersuites for Transport Layer Security (TLS), 2005-12

t. RFC 5246 The Transport Layer Security (TLS) Protocol Version 1.2, 2008-08

u. RFC 5289 TLS Elliptic Curve Cipher Suites with SHA-256/384 and AES Galois Counter Mode (GCM), 2008-08

v. RFC 5929 Channel Bindings for TLS, 2010-07

w. RFC 6358 Additional Master Secret Inputs for TLS, 2012-01

x. RFC 7251 AES-CCM Elliptic Curve Cryptography (ECC) Cipher Suites for TLS, 2014-06

y. RFC 7301 Transport Layer Security (TLS) Application-Layer Protocol Negotiation Extension, 2014-07

z. RFC 7457 Summarizing Known Attacks on Transport Layer Security (TLS) and Datagram TLS (DTLS), 2015-02

[15]. Organization for the Advancement of Structured Information Standards (OASIS) open set of Standards

a. N. Ragouzis et al., Security Assertion Markup Language (SAML) V2.0 Technical Overview, OASIS Committee Draft, March 2008

b. P. Mishra et al. Conformance Requirements for the OASIS Security Assertion Markup Language (SAML) V2.0. OASIS Standard, March 2005.

c. S. Cantor et al. Assertions and Protocols for the OASIS Security Assertion Markup

Language (SAML) V2.0, OASIS Standard, March 2005 
[16]. William List and Rob Melville, IFIP Working Group 11.5, Integrity In Information, Computers and Security, Volume 13, Issue 4, pp. 295-301, Elsevier, doi:10.1016/0167-4048(94)90018-3, 1994.

[17]. William R. Simpson and Coimbatore Chandersekaran, CCCT2010, Volume II, pp. 84-89, "An Agent Based Monitoring System for Web Services," Orlando, FL, April 2011.

[18]. William R. Simpson and Coimbatore Chandersekaran, 1st International Conference on Design, User Experience, and Usability, part of the 14th International Conference on Human-Computer Interaction (HCII 2011), "A Multi-Tiered Approach to Enterprise Support Services," 10 pp. Orlando, FL, July 2011. Also published in: A. Marcus (Ed.): Design, User Experience, and Usability, Pt I, HCII 2011, LNCS 6769, pp. 388-397, 2011.@ Springer-Verlag Berlin Heidelberg 2011.

[19]. William R. Simpson and Coimbatore Chandersekaran, The 3rd International Multi-Conference on Engineering and Technological Innovation: IMETI2010, Volume 2, "Use Case Based Access Control," pp. 297-302, Orlando, FL., July 2010.

[20]. William R. Simpson and Coimbatore Chandersekaran, International Journal of Computer Technology and Application (IJCTA), "An Agent-Based Web-Services Monitoring System," Vol. 2, No. 9, September 2011, pp. 675-685.

[21]. William R. Simpson, Coimbatore Chandersekaran and Ryan Wagner, Lecture Notes in Engineering and Computer Science, Proceedings World Congress on Engineering and Computer Science 2011, Volume I, "High Assurance Challenges for Cloud Computing," pp. 61-66, San Francisco, October 2011.

[22]. Coimbatore Chandersekaran and William R. Simpson, Lecture Notes in Engineering and Computer Science, Proceedings World Congress on Engineering 2012, The 2012 International Conference of Information Security and Internet Engineering, Volume I, "Claims-Based Enterprise-Wide Access Control," pp. 524-529, London, July 2012.

[23]. William R. Simpson and Coimbatore Chandersekaran, Lecture Notes in Engineering and Computer Science, Proceedings World Congress on Engineering 2012, The 2012 International Conference of Information Security and Internet Engineering, Volume I, "Assured Content Delivery in the Enterprise," pp. 555-560, London, July 2012.

[24]. Coimbatore Chandersekaran and William R. Simpson, International Journal of Scientific Computing, Vol. 6, No. 2, "A Uniform Claims-Based Access Control for the Enterprise," December 2012, ISSN: 0973-578X, pp. 1-23.

[25]. William R. Simpson, "Enterprise level security: securing information systems in an uncertain world", Taylor \& Francis (CRC Press), Boca Raton, Florida, @2016, LCCN 2015041818 ; ISBN 978-1-49876445-2, 26 January 2016.

\section{AUTHOR}

Dr. William R. Simpson earned his bachelor of science in aerospace engineering from Virginia Polytechnic Institute and State University, a master of science and a doctor of philosophy in Aeronautical and Astronautical engineering from Ohio State University, and a master of science in administration from George Washington University. He has held academic positions at George Mason University, Old Dominion University, the University of Maryland, and Ohio State University. He has held industry positions at the US Naval Air Test Center, the Center for Naval Analyses, the ARINC Research Corporation, and the Institute for Defense Analyses.

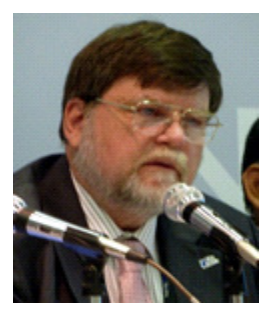




\title{
INVESTIGATING THE IMPLICATION OF BIG DATA ANALYTICS ON CONSUMER FINANCE IN CHINA
}

\author{
Prof. Jiwat Ram ${ }^{1}$, Ding Ronggui ${ }^{1}$ and Hong Yang ${ }^{2}$ \\ ${ }^{1}$ School of Management, Shandong University, Jinan, China \\ ${ }^{2}$ IBSS, XJTLU, China
}

\begin{abstract}
The use of Big Data analytics for business improvements is a vital strategy for survival. In this paper, we report a study that investigates the role of $B D$ analytics on consumer finance, credit card finance in China-a research area that has largely remained unexplored. The largeness and diversity of Chinese consumer market merit an urgent attention and understanding of role of BD analytics is significant both theoretically and managerially. This study achieves that target. Given the exploratory nature of study, we take a qualitative approach. We conduct approximately 30 interviews with baking and finance sector respondents. The data will be recorded, transcribed and translated. We will analyze data using content analysis / thematic analysis technique.
\end{abstract}

\section{KEYWORDS}

Consumer finance; Credit Card finance; Business analytics China

\section{INTRODUCTION}

Big data is another name of large set of massive data that are useful but challenging to process using traditional means (Crosman 2012). Big data analytics refers to the process of extracting useful business information through a combination of software tools and algorithms. With the development of advanced technology, the threshold of processing data has become blur allowing organizations to use a variety of approaches to handle and analyze unstructured and semistructured data (Kugel 2013). It is estimated that there are 1.35 billion customers and 618 million Internet users in China, and the value of China's information data market could be over 3.2 trillion yuan in 2015 (Yao, 2014).

Consumer finance, which refers to short-term loans for personal consumption of durable goods or services has become a promising industry in China (Li 2012). According to Przzom (2012), during 2007 to 2011 period the compound annual growth rate (CAGR) of consumer finance market in China was $28.33 \%$ perhaps due to expanded domestic demand for consumer products. Thus, the development of domestic demand in China could enhance the opportunity for consumer finance market, especially for lending activities, to develop.

Credit card loan is increasingly becoming popular in consumer finance market in China, because of high profit and growth rate. Resultantly banking sector is concentrating heavily on credit card offerings to enhance business performance (Hughes 2016). Although banks in China are eager to

Natarajan Meghanathan et al. (Eds) : ACSIT, SIPM, FCST, CoNeCo, CMIT - 2017

pp. 129- 135, 2017. @ CS \& IT-CSCP 2017

DOI : $10.5121 /$ csit.2017.70810 
collect consumers information to produce tailored products, the lack of reliable consumer credit evaluation and risk management abilities could be an obstacle for further developments of banking sector (China Daily 2004, 2015).

Big data analytics could help banking sector to build and offer innovate financial products and services that meet consumer needs, help improve management of risks of default, make better decisions, and thus enhance business performance (McMalcolm 2015, Manyika et al. 2011, Kambatla et al. 2014, Spotfire Blogging Team 2013). Despite usefulness of BD analytics existing research on the advantages of big data for improvements in consumer finance is in its infancy and practical applications of big data analytics for enhancements in credit card offerings in China remain largely unknown. While some research highlight the performance of commercial banks in using big data analytics, specific research on the operation and use of big data analytics for credit card offerings merit further attention. This research, therefore, fills the gap in knowledge by investigating the implications of big data analytics on consumer finance especially for credit card offerings in China. The research question is:

What are the implications of big data analytics on consumer finance in banking sector such as credit card offerings?

This research is significant in not only investigating an issue in a largely unexplored research strand but also in providing an understanding to managers on how BD analytics can help in developing solutions in consumer finance segment with tailored credit card offerings. The research will be able to build knowledge on how could banking sector differentiate among various consumer groups and gather right consumer data, ensure security of consumers' information, cope with the analytics skill deficiencies and make rational decisions. The results can be used for making long-term strategies for credit card offerings and improvements in business performances with enlarged product portfolios and revenues.

\section{RELATED WORK}

\subsection{Consumer Finance in China}

Consumer finance refers to personal lending activities of durable goods and services, including credit cards, mortgage, and automotive finance (Li 2012). Although consumer finance with low current penetration in China shows a lack of development compared to developed countries, but with a large population combined with urbanization activities consumer finance industry in China is promising industry. As Chinese government is planning to shift GDP growth focus from exports to domestic consumption, consumer lending will become a significant sector (Li 2012).

\subsection{Credit Card offerings in banking sector in China}

Credit card loans are becoming increasingly popular among Chinese with 15.2 trillion yuan credit card payments by 2014 and high volume growth (China Daily, 2015). Credit card loan is a fastgrowing type of loan in consumer finance, with the low initial base, which also implies that this classification of loan will show its potential to grow in the future (Przoom 2012).

Hughes (2016) highlighted the importance by suggesting that credit card loan is a highly profitable project for banks and one way to build loyal credit card customers is to establish strong relationship with consumers assisted by big data analysis. To provide tailored services to customers, to increase credit card holders' portfolio and increase the consumption by cardholders, banks are eager to analyse target consumers' preferences and creditworthiness. However, as China's consumer credit evaluation system and analytics ability is still very nascent and not well- 
developed, banks lack the ability to track records of the financial products they offered to customers, banks might lend too much money to those who have weak creditworthiness (China Daily 2004). In addition, according to a report of China Daily (2015), the rising risks of delay repayment for more than half a year is 42 percent, thus banking sector needs to consider the risk management in credit card loans.

As consumer finance companies and Internet-based financial institutions, the non-banking institutions, fiercely compete to capture the market share, banks should find an efficient approach to enhance businesses performance to maintain market share. In this context, banks have already deployed various strategies such as offering discount on travel routes, offering cash rebates, providing discount on flights, hotel bookings and car rentals, providing various kinds of special treatment to customers, and creating online channels to attract potential customers who own mobile phones (Jiang 2016).

\subsection{Role of Big Data Analytics on Consumer Finance}

According to Kambatla et al. (2014), if a retailer could fully leverage the potential of big data analytics, it could increase its operation margin by at least 60 percent. Big data analytics enables businesses to access and analyze multiple data sources with rapid speed, improve decision making, develop tailored services that meet consumer needs, and obtain better judgement of lending activities thus minimizing losses (McMalcolm 2015).

Using big data analysis could assist banking sector to obtain competitive advantage in many ways. First, it could provide tools and methods for banks to access data and perform analytics in minimum amount of time and easy processes (Manyika et al. 2011). Second, big data analytics could enhance the predictive accuracy of future business plan outcomes by using historical data (Groenfeldt nd). Third, big data analytics allows banks to innovate tailored products that could meet the changing requirements of customers through understanding of specifics of customer behaviours and patterns (Manyika et al. 2011). Fourth, as the market and consumer behaviors involve many uncertainties, banks could use big data analytics to track consumer transactional behaviors and manage risks of default (Spotfire Blogging Team 2013; Fang \& Zhang, 2016). Finally, BD analytics can help banking sector to save cost of tracking and monitoring consumers' information and reduce potential losses from defaulting (Kshetri, 2016).

Particularly with the help of big data analytics, banking sector is able to transform the semistructured and un-structured data into structured data using algorithms and then analyse for business performance improvements (Marr 2016). Banks generate data through multiple channels, such as offline consumption and online transactions (Sen 2014). In this way, banks are able to segment data and track typical customers' financial transaction behaviors across various segmentations to provide personalized services, and build and maintain loyal customer base (Bagchi 2014).

Moreover, a 2010 global fraud study showed that banking and financial services industry dealt with more than 16 percent of fraudulent transactions, thus fraud detection is vital to banking sector development. With advanced BD analytics, banks could scan historical records of consumers to identify abnormal variabilities and even could predict the default risk of consumers, and thus manage market uncertainties and control exposure risk (Pramanick 2013, Marr 2016; Groll et al., 2015).

Despite its advantages, big data analytics also faces many challenges, for instance, choosing the right data sources, the safety of data storage and the gaps in skill for big data analytics (Elan financial services nd, Bagchi 2014). 


\subsection{Big Data implications on Credit Card offerings in China}

Commercial banks, as the leaders of credit market, especially CITIC bank and ICBC (Industrial and Commercial banks of China) in China have pursuing opportunities to building close relationship with consumers. CITIC bank uses SAS (statistical analysis system) and Pivotal Greenplum Database to establish open-platform and improve business performance (Pivotal nd). With the help of advanced data system, CITIC bank is able to provide improve productivity through a customer contact center, maximize marketing campaigns, accelerate data analytics speed and avoid risks. In addition, although ICBC has developed e-payment and online financing system and managed over 100 billion yuan transactions through their e-commerce platform, it is significant for ICBC to use big data analytics to offer suitable financial services (Jiang 2015).

Though banks in China are recognizing the benefits generated from big data applications and are trying to harness the potential, there is still little research that provides specific explanation of how big data analytics is used in credit card offerings for consumer finance in China.

To sum up, based on an extensive review of the literature it could be concluded that the implication of big data on consumer finance such as credit card offerings has not been researched, not just in global banking context but also in Chinese context. Banking and finance is considered vital to economy and society and consumer finance plays a significant role in banking operations, meriting further investigations. Given the gap, we investigate the role and implications of BD analytics on consumer finance through credit card offering from a Chinese perspective.

\section{METHODOLOGY}

Given the exploratory nature of research, we will take a qualitative approach to the study. The research process is divided into three parts - questionnaire design, data collection and data analysis. We will conduct an extensive literature to identify key issues related to credit card finance and construct a semi-structured questionnaire to seek respondents opinions on how BD analytics can help improve the identified issues. Such an approach will help us understand the implications in areas such as classification of consumer groups, approaches that banks could take to analyze transaction data, techniques of using big data analytics to obtain consumers' preference and predict potential risk, the processes that banks use to develop credit card offering strategies. The study will conduct an approximately 30 interviews with target respondents form a variety of occupational backgrounds such as commercial banks' senior managers and financial service industry experts in China. We will use both, online system such as Sojump, or hand-delivered methods to conduct interviews, either face-to-face or by online software (e.g. Skype or WeChat). Interviews will be recorded with respondents' permission, and transcribed. Because the majority of target respondents are Chinese, researcher will translate the Chinese data into English version.

We will analyse the data using Nvivo 10 and content analysis methods. It will enable us to analyze information from interviews, notes of observations with less time and high quality (Hilal and Alabri 2013).

\section{Authors' CONTRIBUtions}

Authors contribute through this study to extend knowledge for both theory and practice in unique ways. Theoretically, the study build fundamental and basic knowledge on the implications of Big Data analytics on consumer finance which is largely unexplored. The theoretical knowledge gained by this study can be used for doing a cross-sectional study to generalize the findings.

The study will also help lay foundation for study of BD analytics in other directions in the banking and finance sector. 
Managerially, the study is an important milestone in providing knowledge on how BD analytics can be used for implementation of credit card based financing in China. The results can be used for driving strategies and plan of action for setting the scene of credit card based innovation offerings. China is a large market and with growing urbanization trends, more and more people will choose credit card based finance. Hence understanding how BD analytics can help in growth of consumer portfolio is important and hence the significance of the study.

\section{LIMITATIONS}

Being the exploratory study, we will be only accessing a small number of respondents. So generalization of results shall be done with caution.

Another limitation is that the limited availability of experts could influence the outcome of the study and generalisation of results. Since most of the respondents will be Chinese so communication and translation of data from Chinese to English language could also entail some limitations.

\section{EXPECTED OUTCOMES}

We expect to establish how BD analytics could be used for improving decision making processes in relation to consumer finance product offerings. The study will focus on understanding the characteristics of products, their features and benefits, ease of use, after sales services and cost of using the products. The results are expected to show the implications for both product / service providers and consumers, thus identifying areas that have synergies and how to exploit those synergies.

The questionnaire will include questions on issues involving how Big Data analytics will help in developing new credit card products, new distribution channels, developing strategies for improving credit card offerings.

\section{CONCLuSion}

While big data analytics may lead to competitive pressure to financial institutions, it also offers opportunities to enhance business performance and expand the efficiency of product / service offerings. Big data implications on consumer finance is expected to be beneficial to financial institutions in improving the relationship between consumers and card issuers, avoiding potential risk of overdue payment, developing innovating products, increasing work efficiency and expanding business scales. Banks in China are more likely to leverage upon the opportunities of big data analytics in establishing online consumer centers and developing insights on consumer behaviors thus improve business administration and long-term credit card supply strategies. This study therefore contributes to enrich our knowledge on big data implication on consumer finance especially for credit card offerings in China

\section{REFERENCES}

[1] Bagchi, S. (2014). Data analytics to play a key role in banks' growth. Available at http://www.cxotoday.com/story/data-analytics-to-play-a-key-role-in-banks-growth/ [Accessed 12 Nov. 2016].

[2] China Daily. (2004). Banks target nation's lucrative consumer finance market. Available at http://www.china.org.cn/english/BAT/90357.htm [Accessed 7 Nov. 2016]. 
[3] China Daily. (2015). China credit card transactions hit 15.2t yuan in 2014. Available at http://www.chinadaily.com.cn/business/2015-07/29/content_21440258.htm [Accessed 7 Nov. 2016].

[4] Crosman, P. (2012). 9 Big data challenges banks face. Available at http://www.americanbanker.com/issues/177_157/nine-big-data-challenges-banks-face-10518181.html [Accessed 7 Nov. 2016].

[5] Elan Financial Services. (nd). Developing a credit card strategy leveraging big data. Available at http://www.creditunions.com/articles/developing-a-credit-card-strategy-leveraging-bigdata/\#ixzz4OSRY2Ggw [Accessed 7 Nov. 2016].

[6] Fang, B., \& Zhang, P. (2016). Big Data in Finance. In Big Data Concepts, Theories, and Applications (pp. 391-412). Springer International Publishing.

[7] Gascon, D. (2015). Getting the most out of big data. Available at http://www.computerworld.com/article/2912441/big-data/getting-the-most-out-of-bigdata.html?nsdr=true\#tk.ctw-infsb [Accessed 7 Nov. 2016].

[8] Goernfedlt, T. (nd). Big data in finance can improve retention and returns. Available at https:/insights.samsung.com/2016/03/28/big-data-in-finance-can-improve-retention-and-returns/ [Accessed 7 Nov. 2016].

[9] Groll, T., O'Halloran, S., Maskey, S., McAllister, G., \& Park, D. (2015). Big Data and the regulation of banking and financial services. Bank Financial Services Policy Report, 34(12), 1-10.

[10] Hilal, A. H. and Alabri, S. S. (2013). Using Nvivo for data analysis in qualitative research. International interdisciplinary journal of education. Vol. 2, No. 2, pp. 181-186.

[11] Hughes, A. M. (2016). How to retain credit card customers. Available at http://www.dbmarketing.com/articles/Art175.htm [Accessed 7 Nov. 2016].

[12] Jiang, X. (2015). ICBC to accelerate internet strategy. Available at http://www.chinadaily.com.cn/business/2015-03/25/content_19909019.htm [Accessed 16 Nov. 2016].

[13] Jiang, X. (2016). Banks fight back in the consumer finance market. Available at http://www.chinadaily.com.cn/bizchina/2016-09/26/content_26892306.htm [Accessed 7 Nov. 2016].

[14] Kambatla, K., Kollias, G., Kurma, V. and Grama, A. (2014). Trends in big data analytics. Journal of parallel and distributed computing. Vol. 74, No. 7, pp. 2561-2573.

[15] Keshetri, N. (2016). Big data's role in expanding access to financial services in China. International journal of information management. Vol. 36, No. 3, pp. 297-308.

[16] Kugel, R. (2013). Finance can get a big advantage from big data. Available at http://www.smartdatacollective.com/robert-kugel/168071/finance-can-get-big-advantage-big-data [Accessed 7 Nov. 2016].

[17] $\mathrm{Li}, \mathrm{J}$. (2012). Consumer finance set to boom in China. Available at http://www.chinadaily.com.cn/business/2012-09/12/content_15751947.htm [Accessed 7 Nov. 2016].

[18] Manyika, J., Chui, M., Brown, B., Bughin, J., Dobbs, R., Roxburgh, C. and Byers, A.H. (2011). Big data: The next frontier for innovation, competition and productivity. Available at http://www.mckinsey.com/business-functions/digital-mckinsey/our-insights/big-data-the-nextfrontier-for-innovation [Accessed 7 Nov. 2016]. 
[19] Marr, B. (2016). Big data in banking: How Citibank delivers real business benefits with its data-first approach. Available at http://www.forbes.com/sites/bernardmarr/2016/09/09/big-data-in-bankinghow-citibank-delivers-real-business-benefits-with-their-data-first-approach/3/\#220f798c13ca [Accessed 12 Nov. 2016].

[20] McMalcolm, J. (2015). Is big data helping improve consumer finance? Available at http://blog.ideacafe.com/big-data-helping-improve-consumer-finance [Accessed 7 Nov. 2016].

[21] Oracle. (2016). An enterprise architect's guide to big data. Available at

[22] http://www.oracle.com/technetwork/topics/entarch/articles/oea-big-data-guide-1522052.pdf [Accessed 7 Nov. 2016].

[23] Pivotal. (nd). China CITIC bank driving revenue and reducing risk. Available at https://pivotal.io/big-data/case-study/china-citic-bank-driving-revenue-and-reducing-risk [Accessed 7 Nov. 2016].

[24] Pramanick, S. (2013). Analytics in banking services. Available at http://www.ibmbigdatahub.com/blog/analytics-banking-services [Accessed 12 Nov. 2016].

[25] Przoom. (2012). BRIC data Publishes Emerging Opportunities in the Chinese Consumer Finance Market. Available at http://www.przoom.com/news/114563/ [Accessed 7 Nov. 2016].

[26] Rouse, M. (2014). Big data analytics. Available at http://searchbusinessanalytics.techtarget.com/definition/big-data-analytics [Accessed 7 Nov. 2016].

[27] Sen, A. (2014). Banking on big data analytics. Available at http://www.livemint.com/Industry/F5uNVbogJfsNB7cSt1 toBL/Banking-on-Big-Data-analytics.html [Accessed 12 Nov. 2016].

[28] Spotfire Blogging Team. (2013). Big data analytics to mitigate risk in banking, insurance. Available at http://www.tibco.com/blog/?p=20587\&utm_source=tuicool\&utm_medium=referral [Accessed 7 Nov. 2016].

[29] Yao, M. (2014). Big opportunities in China to mine big data. Available at http://www.shanghaidaily.com/feature/news-feature/Big-opportunities-in-China-to-mine-bigdata/shdaily.shtml [Accessed 7 Nov. 2016] 


\section{AUTHOR INDEX}

Aleksandar Pluškoski 41

Ding Ronggui 129

Dongok Kang 21

Hong Yang 129

Igor Ciganovic 41

Jelena Vasiljević 41

Jiejun Hu 57

Jiwat Ram 129

Khalid Al-Omar 01

Liuwen $\mathrm{Li} 101$

Minsik Jin 21

Natarajan Meghanathan 89

Parteek Kumar 71

Rupinderdeep Kaur 71

Sharma R.K. 71

Shumin Fei 101

Slađan Kantar 41

Ulisses Ferreira J 11

Wenlin Zou 101

William R. Simpson 115

Xilong Che 57 